\title{
Microbial Utilization of Aqueous Co-Products from Hydrothermal Liquefaction of Microalgae Nannochloropsis oculata
}

\author{
by
}

\author{
Michael Charles Nelson
}

\author{
A dissertation submitted in partial fulfillment \\ of the requirements for the degree of \\ Doctor of Philosophy \\ (Chemical Engineering) \\ in the University of Michigan \\ 2014
}

Doctoral Committee:

Associate Professor Xiaoxia Nina Lin, Co-Chair

Professor Henry Yee-Neen Wang, Co-Chair

Professor Nancy G. Love

Professor Phillip E. Savage, Penn State University 


\section{Acknowledgements}

I would first and foremost like to thank Nina Lin, my advisor, for her mentorship these last five years. She has provided me vast amounts of attention and advice, yet I have also felt appropriately in control of my own projects and plans throughout my doctoral studies. Before returning to graduate school, I was warned that most $\mathrm{PhD}$ advisors are either absent and aloof, or micromanaging and antagonistic, yet I have never seen these qualities in Nina. I was hesitant about leaving a good job and pursuing a doctorate degree in the first place, but became quite confident that it was a good decision after only a semester under her guidance.

My co-advisor, Henry Wang, committee members Phillip Savage and Nancy Love, and the rest of he "Hydrocarbons from Biomass" research group have provided excellent guidance and assistance as well. I had the good fortune to be involved with this group of faculty and graduate students and always felt I was working with collaborators and peers, rather than judges and rivals. The Phillip Savage Lab Group, particularly Peter Valdez and Julia Faeth, have been an invaluable source of collaboration. They are excellent scientists with an amazing work ethic, and I was honored to work with them.

I would like to thank the members of the Lin Group for all of their help over the years, particularly Alissa Kerner and Jeremy Minty for introducing me to all the cellular biology principles I needed to catch up on for my project. Jeremy has been an excellent source of guidance and information and felt almost like a co-advisor at times. 
For the entirety of my graduate studies, I have always had a designated undergraduate student collaborator. The extra efforts in the laboratory by Lian Zhu, Anne Theil, Yan Wu, Mary Guan, Yanxue Gao, Sarah Kistler, and David Boyer have been absolutely crucial for the majority of my work. I wish them the best of luck in their scientific careers, and hope I did not teach them too many bad habits.

Outside of the Chemical Engineering Department, I would like to thank the Civil and Environmental Engineering Department for their help with aqueous chemistry analyses, particularly Thomas Yavaraski. The University of Michigan Sequencing Core and Bioinformatics Core personnel were crucial for my genetic sequencing work, and I would like to thank Richard McEachin, Robert Lyons, Jeanne Geskes, Majusha Pande, and Jim Cavalcoli for their assistance and patience in teaching me about technologies I had so little experience with.

Finally, I would like to thank my family for their support during this time. Sunday dinners with my parents and gatherings with my extended family were a luxury I enjoyed frequently. I would also like to thank the many fellow graduate students I have had the pleasure to socialize with outside of work these past years. A surprising amount of ideas for my thesis work have sprung from concepts studied in completely unrelated work mentioned in casual conversation among my colleagues. These friends have been a source of constant inspiration and occasional inebriation. 


\section{Table of Contents}

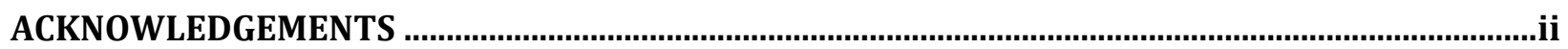

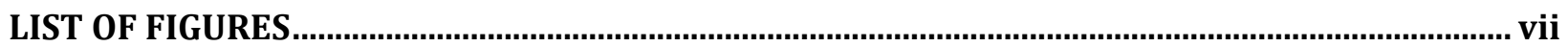

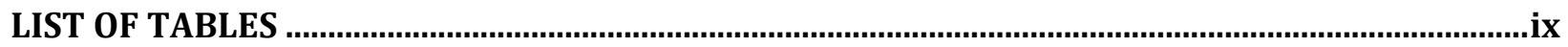

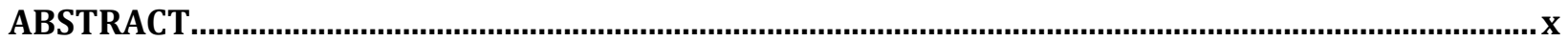

\section{CHAPTER}

1 BACKGROUND AND MOTIVATION..............................................................................................................................

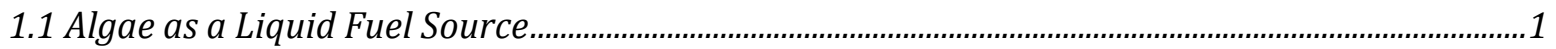

1.1.1 Hydrothermal Liquefaction for "Biocrude" Oil ..........................................................................................................

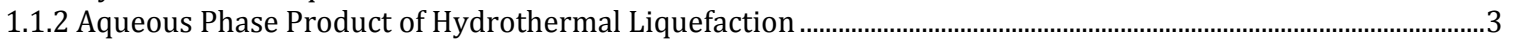

1.2 Microbial Culture on Challenging Substrates...................................................................................5

1.2.1 The Challenges of Cellulosic Biomass Hydrolysate.............................................................................................5

1.3 Genetic Manipulation and Characterization Techniques................................................................... 7

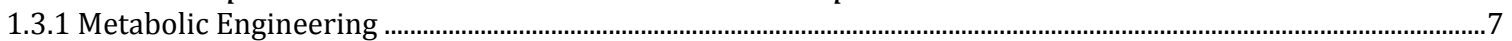

1.3.2 Improvement of Growth and Tolerance Through Evolution ......................................................................................

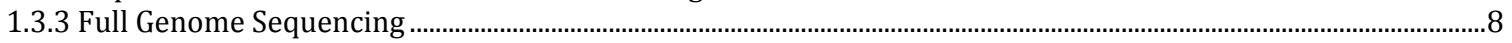

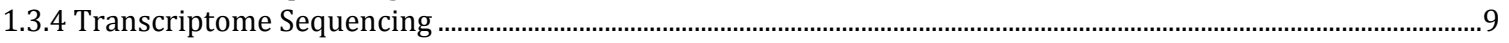

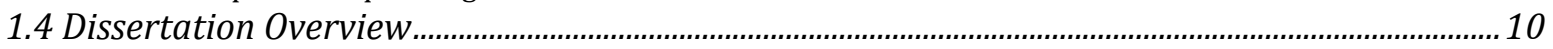

PART I: DETERMINING THE PROPERTIES OF NANNOCHLOROPSIS HTL PRODUCTS AND

CULTURABILITY OF MODEL MICROBES ON THE AQUEOUS PRODUCT ………………….................. 13

2 HYDROTHERMAL LIQUEFACTION OF NANNOCHLOROPSIS SP.: SYSTEMATIC STUDY OF PROCESS VARIABLES AND

ANALYSIS OF THE PRODUCT FRACTIONS ..............................................................................................................13

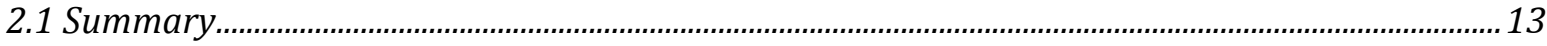

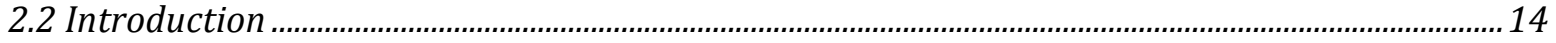

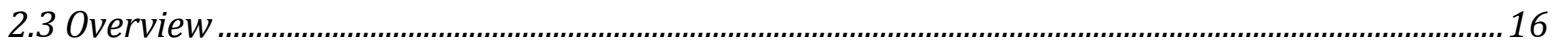

2.4 Results and Discussion .........................................................................................................................16

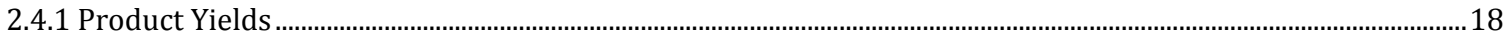

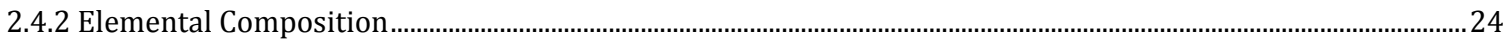

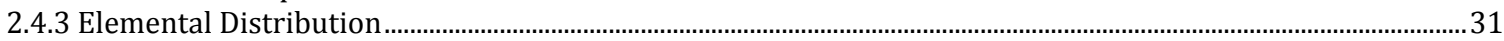

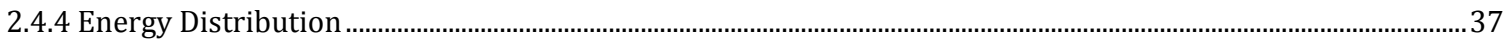

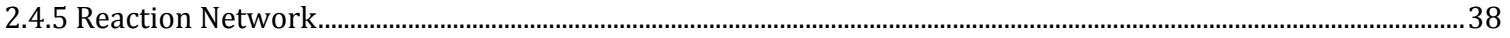

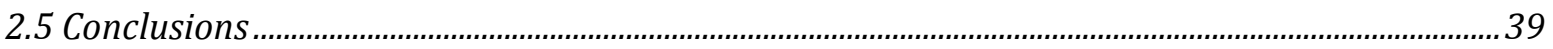

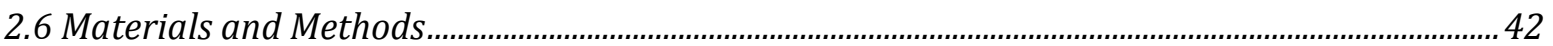

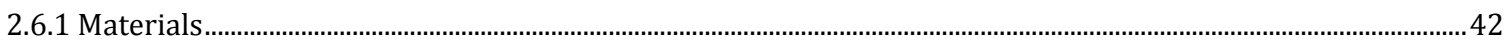

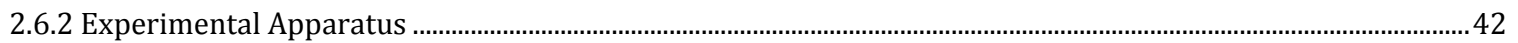

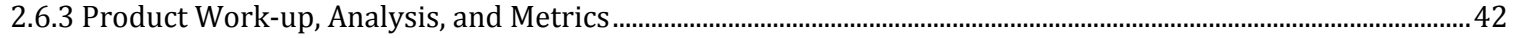

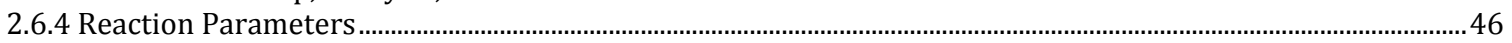

3 MicRobial UTILIZATION OF AQUEOUS CO-PRODUCTS FROM HYDROTHERMAL LIQUEFACTION OF

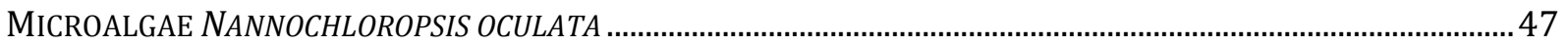

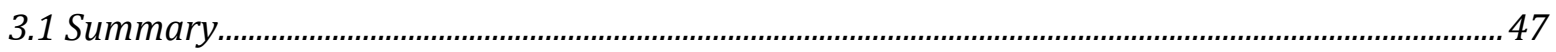

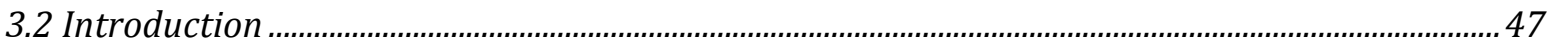

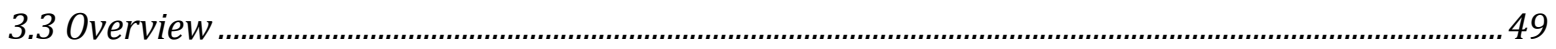

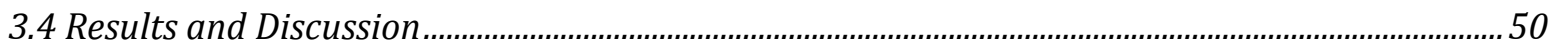

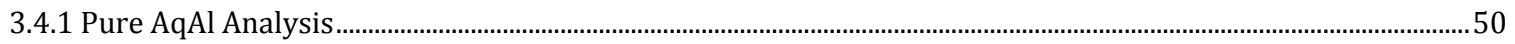




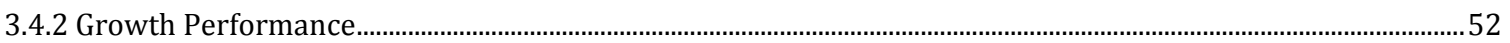

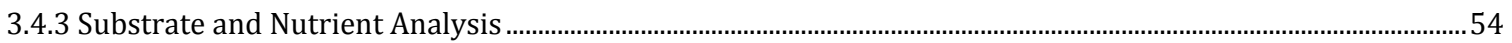

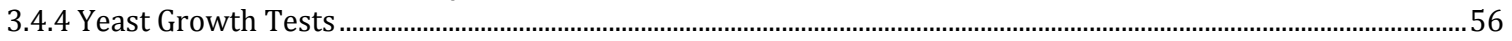

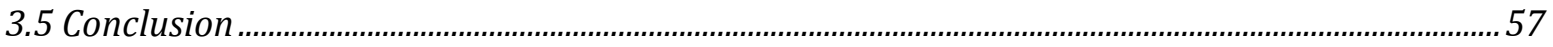

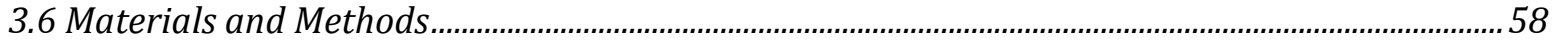

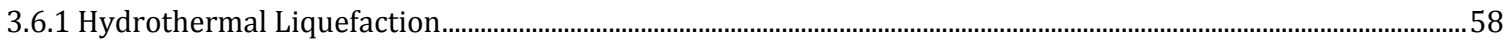

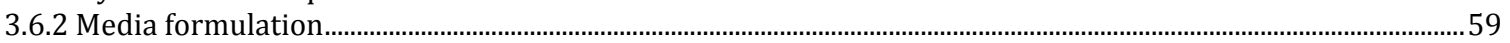

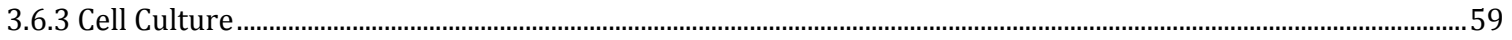

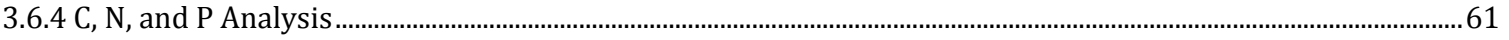

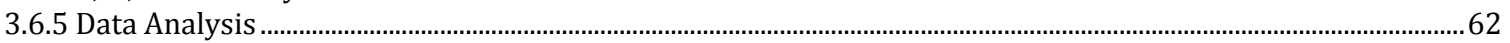

\section{PART II: IMPROVEMENT OF BACTERIAL STRAIN GROWTH ON AQAL THROUGH ADAPTIVE EVOLUTION, AND INVESTIGATION OF TOLERANCE AND SUBSTRATE UTILIZATION} PHENOTYPES THROUGH GENOME AND TRANSCRIPTOME SEQUENCING .................................. 63

4 ADAPTIVE EVOLUTION OF BACTERIA IMPROVES GROWTH ON AQUEOUS CO-PRODUCTS FROM

HYDROTHERMAL LIQUEFACTION OF MICROALGAE NANNOCHLOROPSIS OCULATA ..............................................63

4.1 Summary

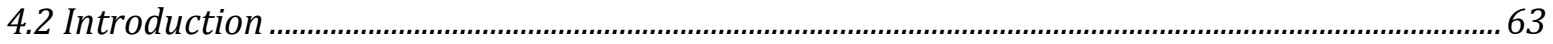

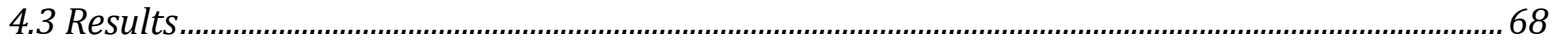

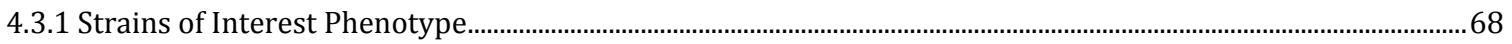

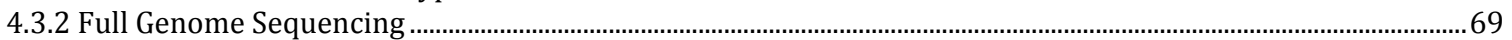

4.3.3 Introduction of Individual SNPs through Homologous Recombination .................................................................73

4.3.4 Growth of Single SNP Strains in AqAl and Minimal Media …..........................................................................74

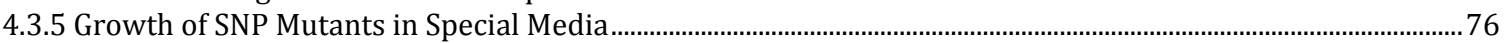

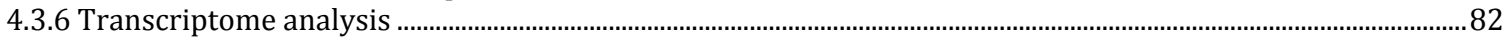

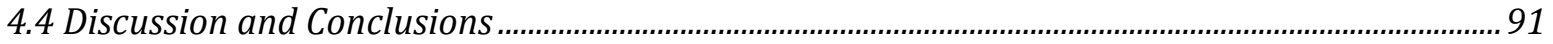

4.5 Materials and Methods .................................................................................................................. 93

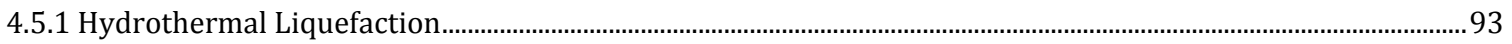

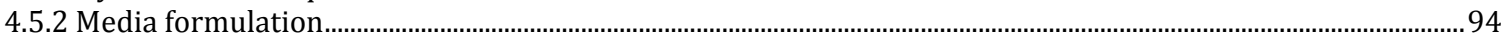

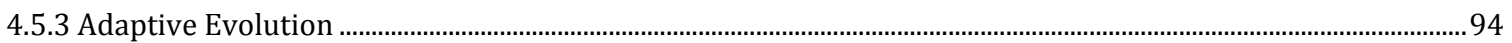

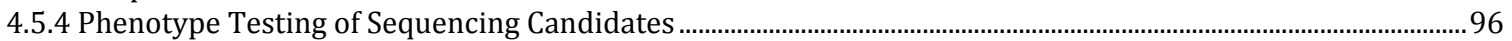

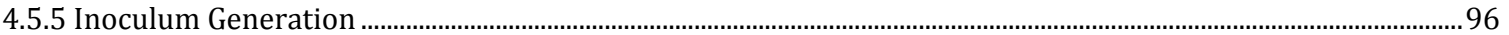

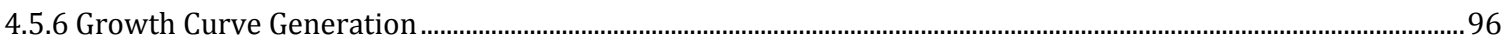

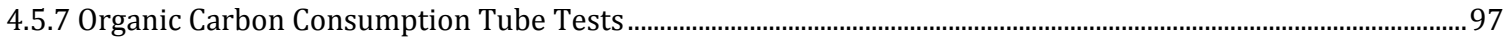

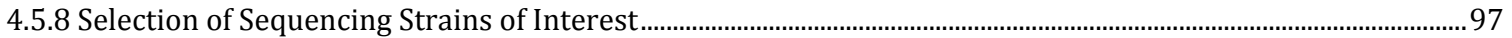

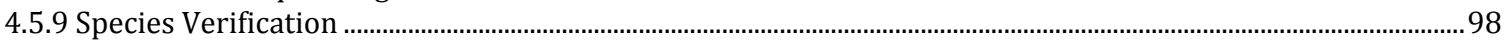

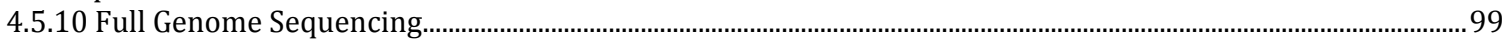

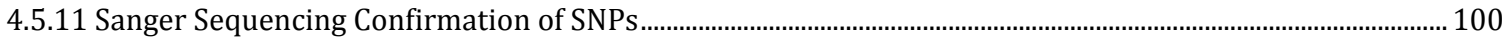

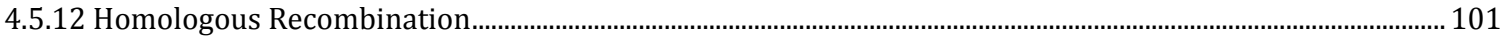

4.5.13 Growth Phenotype Testing of Strains of Interest and Individual Mutants ....................................................... 103

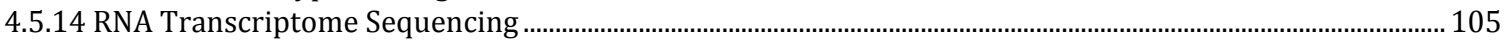

\section{PART III EXAMINING THE UTILITY OF A MICROBIAL SIDE-CULTURE: HYDROTHERMAL LIQUEFACTION OF MICROBIAL BIOMASS AND REGROWTH OF ALGAE ON BACTERIA-}

5 HYDROTHERMAL LIQUEFACTION OF BACTERIA AND YEAST MONOCULTURES................................................ 111

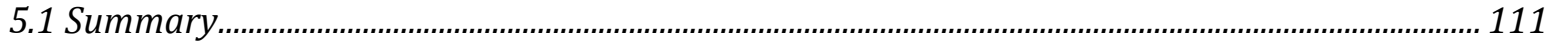

5.2 Introduction

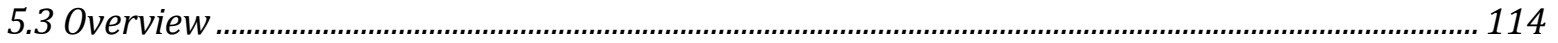

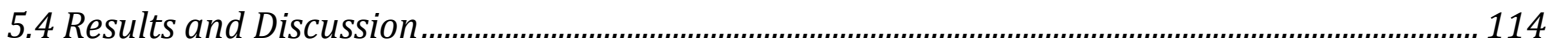

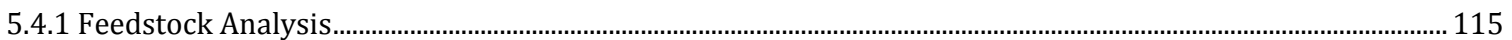

5.4.2 Yields of Product Fractions ..................................................................................................................................................... 116

5.4.3 Elemental Composition of the Light and Heavy Biocrudes ...................................................................................... 119

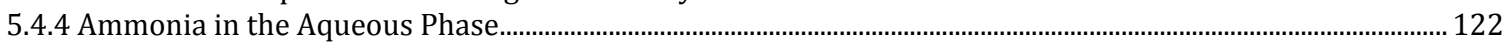

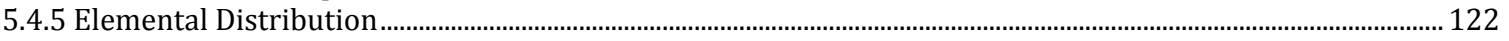

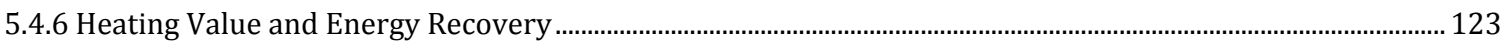

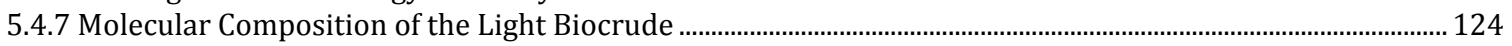




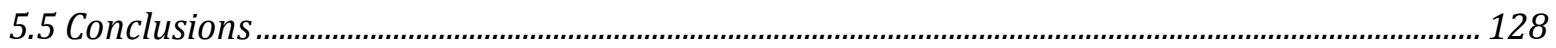

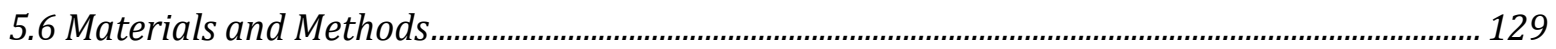

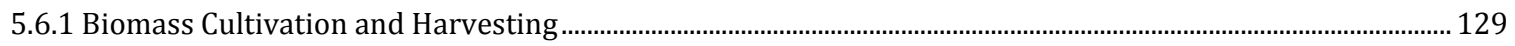

5.6.2 Biomass Feedstock Analysis .......................................................................................................................................... 130

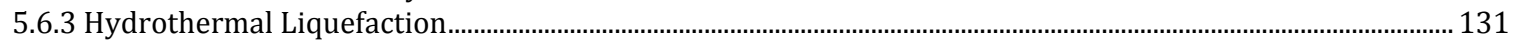

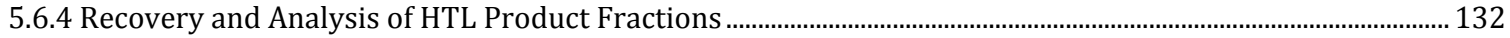

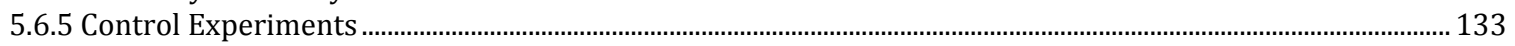

6 GrowTH OF ALGAE ON AQAL PRE-PRoCESSED BY BACTERIAL CULTURE.................................................... 135

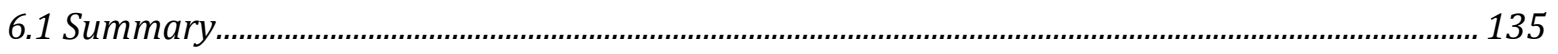

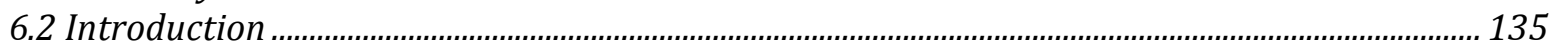

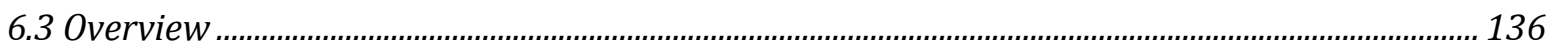

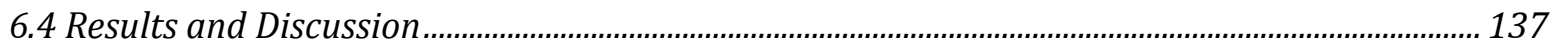

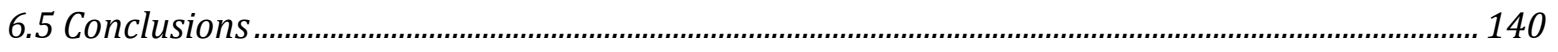

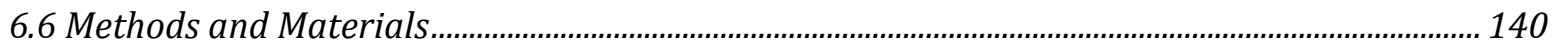

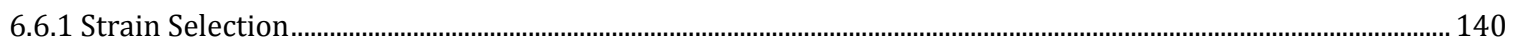

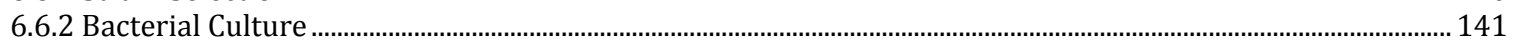

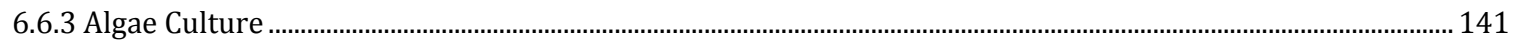

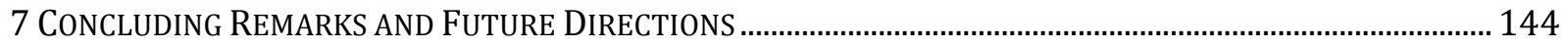

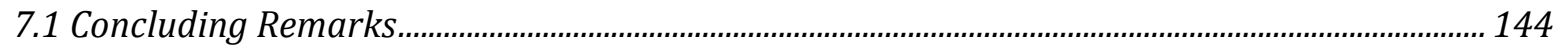

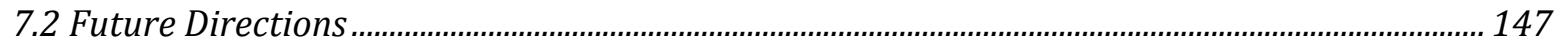

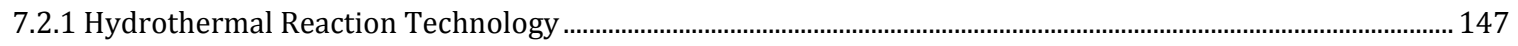

7.2.2 AqAl Characterization ............................................................................................................................................................ 147

7.2.3 Next Generation Sequencing Technology ………………..................................................................................... 147

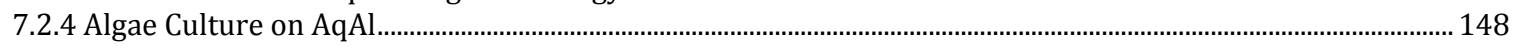

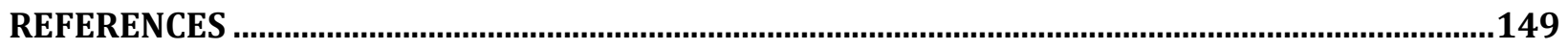




\section{List of Figures}

FIGURE 1.1 HTL PRODUCT YIELDS FROM MULTIPLE PURE CARBOHYDRATES/PROTEINS/FATS AS WELL AS WHOLE ALGAE BIOMASS.

FIGURE 1.2 THE DECLINING COST OF SEQUENCING PER MEGABASE PAIR OF DNA. ……................................................................ 9

FIGURE 1.3 PROPOSED INTERMEDIATE MICROBIAL CULTURE FOR ALGAE BIO-OIL PRODUCTION PROCESS ...................................... 10

FIGURE 2.1 PROCEDURE FOR REACTION AND PRODUCT WORK-UP ................................................................................................ 16

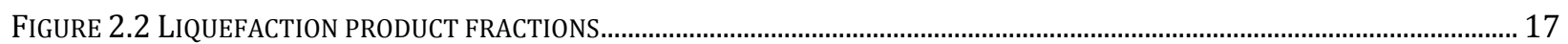

FigURE 2.3 YIELD OF A) BioCRUDE, B) LIGHT BIOCRUDE, AND C) HEAVY BIOCRUDE ................................................................... 20

Figure 2.4 Yield of A) Aqueous-PHASE ProductS, B) Water-Soluble ProductS, AND C) Volatiles................................ 20

FIGURE 2.5 YIELD OF A) GAS AND B) SOLIDS.......................................................................................................................... 22

FigURE 2.6 YIELDS OF BIOCRUDE (†), LIGHT BIOCRUDE ( $(\bullet)$, HEAVY BIOCRUDE $(\bullet)$, AQUEOUS-PHASE PRODUCTS AND GAS $(\bullet)$, AND SOLIDS ( $\mathbf{\Delta}$ ) PRODUCED AT $350^{\circ} \mathrm{C}$ FOR 60 MIN ……........................................................................................ 23

FIGURE 2.7 ELEMENTAL COMPOSITION A) C, B) N, C) S, AND D) O IN THE LIGHT BIOCRUDE …………………............................ 25

FiguRE 2.8 ELEMENTAL COMPOSITION A) C, B) N, C) S, AND D) O IN THE HEAVY BIOCRUDE …................................................... 27

FigURE 2.9 CONCENTRATION OF C (A) AND \% OF C PRESENT AS INORGANIC CARBON (B) IN THE AQUEOUS PHASE...................... 28

FIGURE 2.10 CARBON DISTRIBUTION IN A) IN THE AQUEOUS PHASE AND B) THE WATER-SOLUBLE PRODUCTS ..............................32

FigURE 2.11 FIGURE 11. PERCENT OF TOTAL NITROGEN AS AMMONIA IN THE AQUEOUS PHASE..................................................... 35

FIGURE 2.12 TEMPORAL VARIATION OF YIELDS OF LIGHT BIOCRUDE $(\bullet)$, HEAVY BIOCRUDE $(\boldsymbol{\bullet})$, AQUEOUS-PHASE PRODUCTS

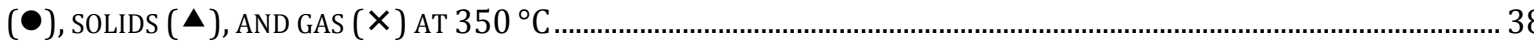

FIGURE 2.13 POTENTIAL REACTION NETWORK FOR HYDROTHERMAL LIQUEFACTION OF ALGAE.................................................... 39

FIGURE 3.1 EXPERIMENTAL FLOWCHART FOR BACTERIAL GROWTH STUDIES ILLUSTRATING MATERIAL GENERATION STEPS AND THE DATA ACQUIRED FROM EACH CULTURE STUDY. ....................................................................................................... 50

FIGURE 3.2 FINAL CELL DENSITIES MEASURED BY $\triangle \mathrm{OD}_{650}$ (SAMPLE OD MINUS MEDIUM BLANK OD) OF 7 ML CULTURE

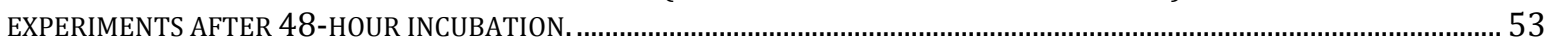

FIGURE 3.3 GROWTH RATE RESULTS FROM MICROPLATE CULTURE TEST.................................................................................. 54

FIGURE 3.4 CARBON CONSUMPTION DATA FROM 7 ML, 48-HOUR CULTURE EXPERIMENTS. ........................................................ 56

FigURE 3.5 FINAL $\Delta \mathrm{OD}_{650}$ OF 2 ML S. CEREVISIAE CULTURES AFTER 48-HOUR INCUBATION IN GLUCOSE-SPIKED MEDIA. ........... 57

FIGURE 4.1 PROJECT DESIGN ...................................................................................................................................... 67

FigURE 4.2 E. Coli (A) AND P. PUTIDA (B) STRAINS OF INTEREST GROWN IN 30 vol\% AQAL MEDIUM................................... 69

Figure 4.3 MAXIMUM OPTICAL DENSiTIES (A, C) AND OVERALl GROWTH RATES (B, D) OF E. COLI AND P. PUTIDA STRAins OF INTEREST WHEN GROWN IN 30 VOL\% AQAL....................................................................................................... 69

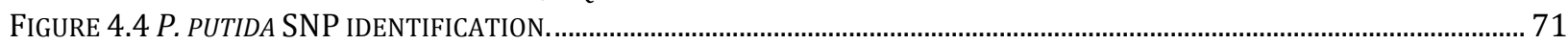

FIGURE 4.5 E. COLI SNP IDENTIFICATION.................................................................................................................... 72

FIGURE 4.6 GROWTH DATA FOR SINGLE-SNP STRAINS AND PARENT ECHW24 STRAIN (BLACK) GROWN IN 30 VOL\% AQAL MEDIA (A) AND COMPLETE MOPS MEDIA (B)..................................................................................................... 75

FIGURE 4.7 MAX OD (A, C) AND OGR (B, D) OF SINGLE-SNP MUTANT STRANS AND PARENT ECHW24 STRAIN GROWN IN 30 VOL\% AQAL MEDIA AND COMPLETE MOPS MEDIA................................................................................................ 75

FIGURE 4.8 GROWTH OF GLPK MUTANTS AND PARENT STRAIN IN MOPS MINIMAL MEDIA CONTAINING DIFFERENT CONCENTRATIONS OF GLUCOSE (GLU) AND GLYCEROL (GLY).................................................................................... 77

FIGURE 4.9 MAX OD (A) AND OVERALL GROWTH RATE (B) OF GLPK MUTANTS AND PARENT STRAIN IN MOPS MINIMAL MEDIA CONTAINING VARIOUS CONCENTRATIONS OF GLUCOSE AND GLYCEROL ……………….................................................... 77

FIGURE 4.10 GROWTH OF PTSP AND PARENT STRAIN IN MEDIA WITH VARYING C:N RATIOS........................................................ 79

FIGURE 4.11 MAXIMUM OPTICAL DENSITY (A) AND OGR (B) OF PTSP MUTANT AND PARENT STRAINS GROWING IN MOPS MINIMAL MEDIA WITH VARYING C:N RATIOS......................................................................................................... 79

FigURE 4.12 GROWTH OF PARENT STRAIN AND ILVH/ILVI MUTANTS IN COMPLETE MOPS MEDIUM (A), AND COMPLETE MOPS MEDIUM SPIKED WITH 100 PPM (B) AND 500 PPM(C) IMAZAQUIN.......................................................................... 81

FigURE 4.13 MAXIMUM ODS (A) AND OVERALL GROWTH RATES (B) OF ILV MUTANTS AND PARENT STRAIN GROWN IN

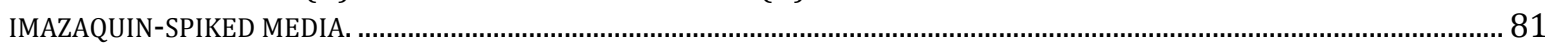


FIGURE 4.14 NUMBER OF UNIQUE AND SHARED DIFFERENTIALLY EXPRESSED GENES IN DIFFERENT COMPARISONS.

FIGURE 5.1 YIELDS OF LIGHT AND HEAVY BIOCRUDE PRODUCT FRACTIONS (WT \% DAF) FOR EACH BIOMASS AND ISOTHERMAL

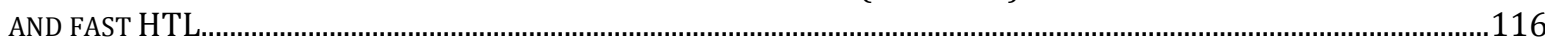

FIGURE 5.2 AVERAGE COMPOSITION OF THE GAS PHASE FROM ISOTHERMAL AND FAST HTL...................................................119

FIGURE 5.3 COMPOSITION OF A) C, B) N, C) O, AND D) S IN THE LIGHT AND HEAVY BIOCRUDES ...............................................121

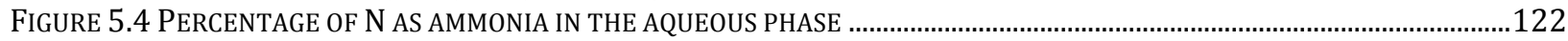

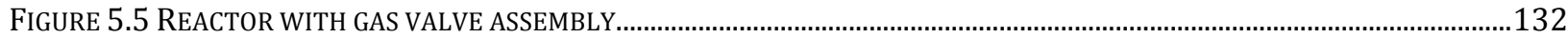

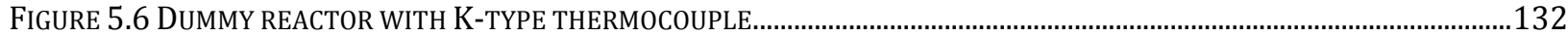

FIGURE 6.1 PLAN FOR INVESTIGATING THE EFFECTS OF BACTERIAL PRECULTURE ON ALGAE REGROWTH .................................137

FIGURE 6.2 FLUORESCENCE MEASUREMENTS OF ALGAE CULTURES GROWN IN UNPROCESSED AQAL COMBO MEDIA IN TWO

MICROPLATE STUDIES: WIDE RANGE (A) AND HIGH CONCENTRATION (B)....................................................................139

FIGURE 6.3 GROWTH OF C. VULGARIS IN COMBO MEDIA WITH 0.5 VOL\% (A), 3 VOL\% (B), AND 6 VOL\% (C) AQAL PROCESSED

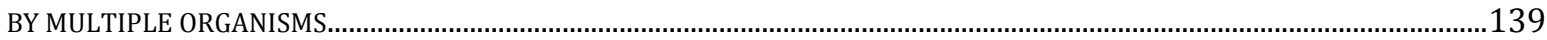

FIGURE 7.1 PROCESS PLOW CHARTS FOR THE TWO THEORETICAL PROCESSES ANALYZED BY NOLAN ET AL …...........................145

FIGURE 7.2 RESULTS FROM NOLAN ET AL SHOWING FOUR PROCESS METRICS FOR TWO HTL CONDITIONS...............................146 


\section{List of Tables}

TABLE 1.1 NitROGENOUS ORGANIC COMPOUNDS WITH SUSPECTED CYTOTOXICITY FOUND IN AQAL FROM SPIRULINA REACTED AT $300{ }^{\circ} \mathrm{C}$ FOR 30 MINUTES.

TABLE 2.1 AVERAGE INITIAL CONTENT OF PRODUCT FRACTIONS IN THE FEEDSTOCK.................................................................. 18

TABLE 2.2 TABLE 2. N CONCENTRATION IN THE AQUEOUS PHASE (G/L) ................................................................................. 30

TABLE 2.3 CARBON AND NITROGEN COMPOSITION (WT \%) OF THE SOLIDS ................................................................................. 30

TABLE 2.4 CARBON (\%) DISTRIBUTED TO THE LIGHT AND HEAVY BIOCRUDES.............................................................................. 31

TABLE 2.5 NITROGEN (\%) DISTRIBUTED TO THE LIGHT AND HEAVY BIOCRUDES.......................................................................... 33

TABLE 2.6 NITROGEN (\%) DISTRIBUTED TO THE AQUEOUS PHASE............................................................................................. 34

TABLE 2.7 PHOSPHORUS (\%) DISTRIBUTED TO THE AQUEOUS PHASE .......................................................................................... 36

TABLE 2.8 BIOCRUDE ENERGY METRICS AT DIFFERENT CONDITIONS...................................................................................... 37

TABLE 3.1 CONCENTRATIONS OF ALL MEASURED CHEMICALS IN AQAL ……………………............................................. 52

TABLE 4.1 THE MOST DIFFERENTIALLY EXPRESSED GENES IN THE MUTANT WHEN GROWN IN 30 VOL\% AQAL MEDIUM (IN

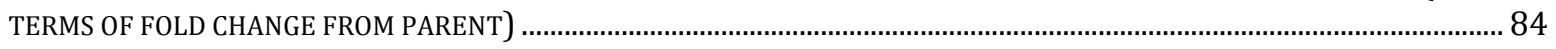

TABLE 4.2 METABOLIC PATHWAYS WITH HIGHER EXPRESSION (TOP) AND LOWER EXPRESSION (BOTTOM) IN K2C2 WHEN COMPARED TO THE PARENT K12 STRAIN WHEN GROWN IN 30 VOL\% AQAL MEDIA.......................................................... 86

TABLE 4.3 PATHWAYS DIFFERENTIALLY EXPRESSED BETWEEN COMPLETE MOPS AND 30 VOL\% AQAL MEDIA GROWTHIN IN K2C2

TABLE 4.4 SELECTION DATA FOR STRAINS OF INTEREST FROM SEQUENCING CANDIDATES …....................................................109

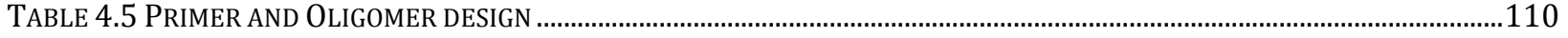

TABLE 5.1 ELEMENTAL (WT \%), BIOCHEMICAL COMPOSITION (WT \%), AND HHV (MJ/KG) OF THE BIOMASS .........................115

TABlE 5.2 YIELDS OF SOLID, AQUEOUS-PHASE, AND GAS PRODUCT FraCTIONS (WT \% DRY BASIS) …….................................118

TABLE 5.3 HigHER HEATING VALUE AND ENERGY RECOVERY OF THE BIOCRUDE....................................................................123

TABLE 5.4 TENTATIVE IDENTITIES AND RELATIVE ABUNDANCE OF DIFFERENT COMPOUNDS IN THE LIGHT BIOCRUDE...............125 


\section{Abstract \\ Microbial Utilization of Aqueous Co-Products from Hydrothermal Liquefaction of Microalgae Nannochloropsis oculata}

by

\section{Michael Charles Nelson}

\section{Co-chairs: Xiaoxia (Nina) Lin and Henry Wang}

Algae biomass is a promising source of liquid biofuels. Hydrothermal liquefaction can convert wet algae biomass into a "biocrude" oil with similar properties to crude petroleum, but it also produces an aqueous co-product (AqAl) that contains most of the nitrogen and phosphorus from the initial biomass and up to $40 \%$ of the carbon. Efficient recycle of these components within the system is crucial, yet direct feeding of AqAl back to algae ponds has proven problematic. In this work, we investigate the use of an intermediate bacterial culture to initially utilize this challenging product.

In the first part, we investigate the properties of products formed from Nannochloropsis oculata algae biomass processed under a variety of hydrothermal reaction conditions. We then use AqAl as the sole C/N/P source for bacterial culturability studies on bacteria Escherichia coli and Pseudomonas putida. We determine that bacterial culture is feasible and the bacterial strains studied can utilize up to $43 \%$ of the organic carbon present. However, there is an inhibitory effect imposed by the AqAl.

In the second part, we improve the bacterial strains' growth phenotypes through adaptive evolution in media of increasing AqAl concentration. Evolved E. coli and P. putida strains can grow at rates $104 \%$ and $260 \%$ faster and reach cell concentrations $24 \%$ and $61 \%$ higher, respectively, in AqAl media. The full genomes of improved strains are determined and mutations conferring benefits are identified. Some of these mutations are individually introduced to E. coli to investigate their specific effects. Also, gene expression levels between a top-performing evolved strain of $E$. coli and its parent strain are compared. 
Finally, we investigate the utility of a microbial processing step on an integrated algae biofuel process in two ways. First, we investigate the possibility of using microbial biomass as a supplemental feedstock for hydrothermal reactions, observing oil yields upwards of $75 \%$ of that of algae. Second, we investigate the effects bacterial pre-culture has on AqAl pertaining to its suitability as an algae growth medium component.

We conclude that an intermediate bacterial culture could contribute to the overall efficiency and sustainability of an algae biofuel process. 


\section{Chapter 1}

\section{Background and Motivation}

\subsection{Algae as a Liquid Fuel Source}

Environmental concerns and the steady increase in the cost of oil have lead to a rise in funding for alternative energy research in recent years. Wind, solar, and fuel cell based energy are all promising prospects for sustainable electricity production. For transportation applications, however, all of these technologies are incompatible with the current fuel distribution system and would require a substantial infrastructure investment to implement on a national level. Biologically derived liquid fuels hold promise in this area since they can be directly integrated into the current liquid hydrocarbon distribution infrastructure, and even be mixed with petroleum-based fuels [1].

As a source of biomass, micro-algae offer several advantages compared to terrestrial plant species. First-generation biofuels such as corn-ethanol exert a large strain on food supply. Second-generation biofuels, such as alcohols from ligno-cellulosic biomass (e.g. switch grass) fermentation address this problem somewhat, but still compete with food crops for arable land [2]. This type of biomass also requires hydrolysis of cellulose and hemicellulose in order to liberate fermentable sugars, and while much progress has been made in the past decade in this area, hydrolysis still remains one of the most costly and problematic steps in the process [3]. Algae do not compete with food crops for arable land or fresh water and can theoretically be grown with much higher annual yields per acre. Algae also lack an abundance of rigid cellulose and lignin structures whose enzymatic breakdown has proven a major roadblock in terrestrial biomass processing [1].

However, algae cultivation for use as a fuel feedstock still poses several key challenges. While more algae can be grown per unit land area than terrestrial crops, algae still require similar quantities of nitrogen, phosphorous, and micronutrient fertilizers, many of which are energy-intensive to produce. Recent life cycle analysis studies have 
concluded that algae fuel theoretical cost is heavily influenced by fertilizer production energy requirement and a process fertilizer recycle efficiency [4]-[6].

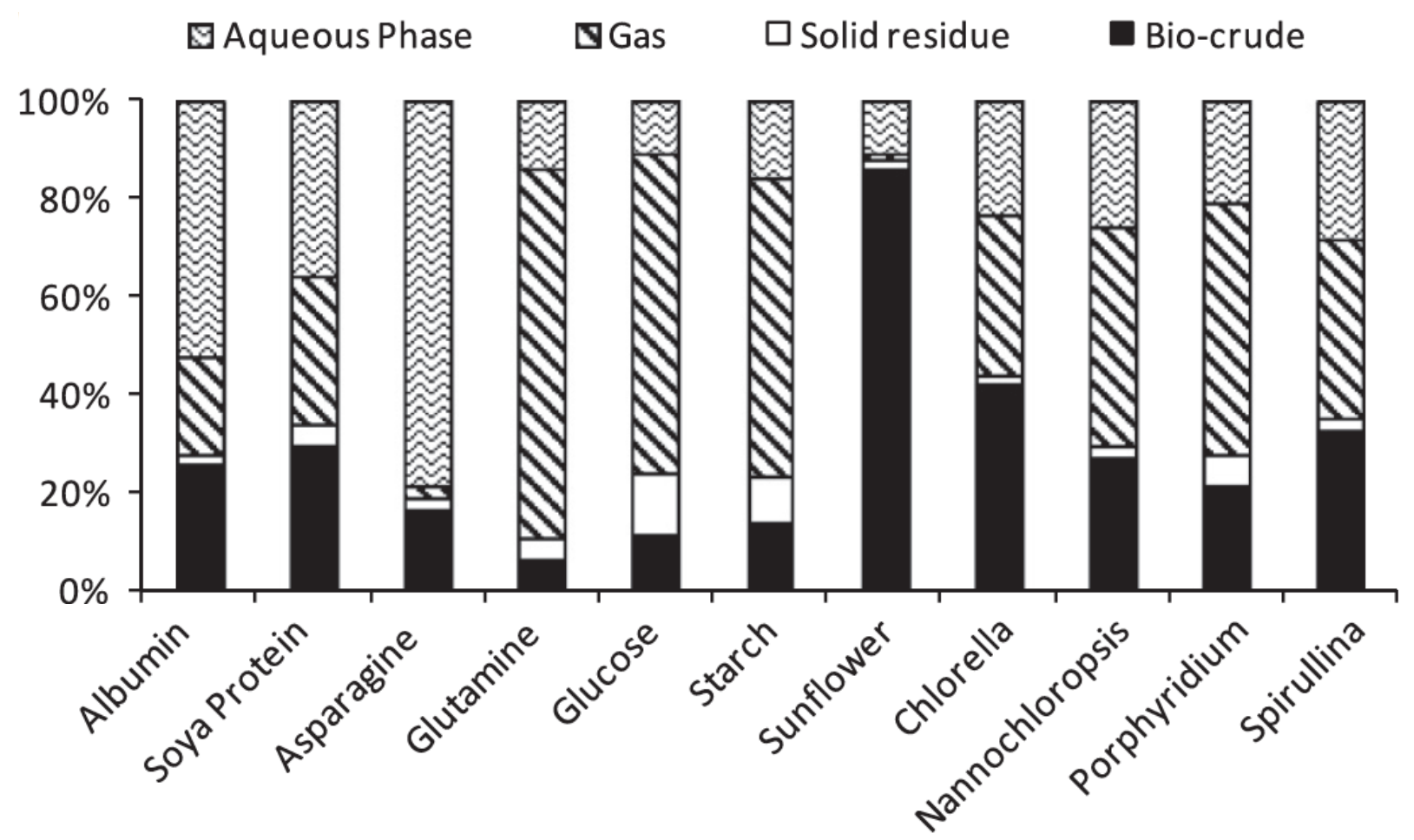

Figure 1.1 HTL product yields from multiple pure carbohydrates/proteins/fats as well as whole algae biomass. Yields are in terms of wt\% of initial feedstock. Figure adapted from [7].

\subsubsection{Hydrothermal Liquefaction for "Biocrude" Oil}

Initial investigation into algae biofuel proposed the production of biodiesel through transesterification of algae lipids. This entails an energy-intensive extraction of lipids and the upgrade of only this biomass fraction into a fuel, neglecting carbohydrate and protein fractions. Furthermore, there is an antagonistic relationship between algae growth conditions promoting high lipid content (nitrogen limitation) and those promoting fast growth rate (nitrogen and other nutrient access) [8]. An alternative technology is hydrothermal liquefaction (HTL), the heating of wet algae biomass to produce a "biocrude" oil with similar properties to crude petroleum. This process transforms a fraction of all biomass components into the oil product (Figure 1.1) [7]. Multiple life cycle analysis studies have concluded that HTL offers distinct advantages over lipid extraction and transesterification through more efficient use of biomass and less overall energy consumption [6], [9], yet these same studies also stress that nutrient (particularly nitrogen 
and phosphorus) recycle efficiency within the HTL system (a metric with little published data available) is an important factor that could affect HTL's economic feasibility and material sustainability.

\subsubsection{Aqueous Phase Product of Hydrothermal Liquefaction}

While the biocrude oil produced by HTL contains the majority of the chemical energy from the initial biomass, the aqueous phase product of the algae biomass (AqAl) contains the majority of the nitrogen and phosphorus [7], [10]. 40\% to $80 \%$ of the phosphorus is present, almost entirely in the form of orthophosphate [11]. Between $66 \%$ and $80 \%$ of the nitrogen in the biomass is present and $20 \%$ to $60 \%$ of it is in the form of ammonia [11]. Efficient recycle of AqAl nutrients within the biofuel production process is important, and initial efforts to reefed it to algae ponds met with limited success. Minowa et al and Tsukahara et al showed algae could be regrown on AqAl, utilizing ammonia as a nitrogen source, but at roughly one eighth the growth rate achieved in ideal media. They were also using a slightly different process of low-temperature gasification instead of HTL [12], [13]. Further work was carried out by Jena et al and Biller et al using actual HTL and investigating several different algae species. They found algal growth was possible at an ideal HTL dilution below roughly 1:200 (0.5 vol\% AqAl in water) at a rate and yield similar, but still lower than ideal media. Growth in conditions above 0.5 vol\% AqAl seemed to be hampered by inhibitory effects, with slower growth rates and less efficient nutrient uptake. Both works suspected phenolic compounds to be contributing to this inhibition[14], [15]. Pham et al identified specific toxic nitrogenous organic compounds in AqAl (Table 1.1) yet these are only some of the hundreds of unknown organic compounds in the product and there is no definite link between any one chemical species and the apparent toxicity of AqAl [16]. 
Table 1.1 Nitrogenous Organic Compounds with suspected cytotoxicity found in AqAl from Spirulina reacted at $300{ }^{\circ} \mathrm{C}$ for 30 minutes. Adapted from [16].

\begin{tabular}{|c|c|c|c|c|}
\hline Compound name & Structure and formula & MW & CAS \# & Conc. (ppm) \\
\hline $\begin{array}{l}\sigma \text {-valerolactam } \\
\text { or 2-piperidone }\end{array}$ & $\mathrm{C}_{5} \mathrm{H}_{9} \mathrm{NO}$ & 99.13 & $675-20-7$ & 139 \\
\hline$\varepsilon$-caprolactam & $\mathrm{C}_{6} \mathrm{H}_{11} \mathrm{NO}$ & 113.16 & $105-60-2$ & 10 \\
\hline 2,6-dimethyl-3-pyridinol & $\mathrm{C}_{7} \mathrm{H}_{9} \mathrm{NO}$ & 123.15 & $1122-43-6$ & 8.2 \\
\hline $\begin{array}{c}\text { 2,2,6,6-tetramethyl- } \\
\text { 4-piperidone }\end{array}$ & & 155.24 & $826-36-8$ & 3.5 \\
\hline 1- methyl-2-pyrrolidinone & $C_{5 H} 1 \mathrm{~N}$ & 99.13 & $872-50-4$ & 1.5 \\
\hline $\begin{array}{l}\text { 2-pyrrolidinone } \\
\text { or butyrolactam }\end{array}$ & $\mathrm{C}_{4} \mathrm{H}_{7} \mathrm{NO}$ & 85.1 & $616-45-5$ & 0.82 \\
\hline 3-dimethylamino-phenol & & 137.18 & $99-07-0$ & 0.37 \\
\hline pyridine & $\mathrm{C}_{5} \mathrm{H}_{5} \mathrm{~N}$ & 79.1 & $110-86-1$ & 0.16 \\
\hline $\begin{array}{l}\text { 2-picoline } \\
\text { or 2-methylpyridine }\end{array}$ & $\mathrm{C}_{6} \mathrm{H}_{7} \mathrm{~N}$ & 93.13 & $109-06-8$ & 0.052 \\
\hline
\end{tabular}


Progress has been made in AqAl recycle strategies recently, the most notable being work done by Garcia-Alba et al regrowing algae in diluted AqAl spiked with various ideal media components and achieving very similar growth to normal ideal medium. They concluded that toxicity of AqAl had been overestimated and that lack of essential bioavailable micronutrients was more to blame for reduced algae growth in AqAl. However, there were still noticeable issues with algae growth on AqAl, such as the algae's inability to utilize nitrogen tied up in various organic sources [17].

One major factor to consider when comparing research on HTL, however, is that the yield and composition of all the products formed, including the AqAl, varies immensely due to many contributing factors. Algae species [7], reaction time and temperature [11], [18], use of a catalyst or specific gas in the reactor headspace [19], biocrude extraction technique [20], and AqAl preparation/filtration [16] all vary the properties of HTL products significantly. Furthermore, studies concerning reefed of AqAl to algae growth can use one strain of algae with readily purchasable, concentrated biomass as the HTL feedstock, while using different species that are easier/quicker to grow in a laboratory setting for the regrowth tests [14]. All these factors make it quite difficult to compare individual studies to one another and develop a firm definition of what AqAl is composed of and its suitability as an algae growth medium.

\subsection{Microbial Culture on Challenging Substrates}

Algae regrowth on the aqueous phase from hydrothermal liquefaction of biomass is a very specific and rather recent challenge with little published research pertaining to it. There are other more developed industries with much larger bodies of work that deal with the resistance of growth inhibitors and metabolism of substrates available in cellular breakdown products. The area primarily used as inspiration for this thesis is bacterial/fungal utilization of cellulosic feedstock hydrolysates for biofuel production.

\subsubsection{The Challenges of Cellulosic Biomass Hydrolysate}

The hydrolysis of cellulosic biomass yields three main classes of microbial growth inhibitors: furans, weak acids, and phenolics. Research has been ongoing for over two 
decades to design bacterial and fungal strains that resist these classes of compounds while utilizing the pentose and hexose sugars present for fermentation of biofuel alcohols.

Furans, particularly furfural and 5-hydroxymethyl-2-furaldehyde (HMF), cause damage to cellular components, including mitochondrial and vacuole membranes, the actin cytoskeleton, and DNA, by causing buildup of reactive oxygen species within the cell [21]. This can be countered by overexpression of genes involved in producing nucleotides for DNA repair [22] or by expressing enzymes to reduce furans to less toxic alcohol species [23], though the latter results in growth inhibition via NADPH depletion.

Phenolics, compounds in cellulosic biomass hydrolysate but also found in AqAl, are a diverse group of chemicals with no one general mechanism for their toxic properties, and different individual compounds can have minimum inhibitory concentrations that vary by orders of magnitude [24]. Their effects seem to be addressed by active evacuation through efflux pumps [25] and expression of general oxidative stress response genes [26].

Weak acids, such as acetic acid (a compound found to be a significant fraction of the organic carbon in AqAl [17]) interfere with cell wall function, lipid metabolism, and nucleotide synthesis [27]. Overexpression of genes in the non-oxidative pentose phosphate pathway can increase tolerance of acetic acid [28].

Many inhibitor compounds in cellulosic biomass hydrolysate can have a synergistic effect on microbial toxicity that is greater than the sum of the individual inhibitors [29]. For example, phenols and acetic acid have a synergistic toxic effect on various bacteria species. Thymine supplementation can alleviate this effect specifically for phenol exposure, while uracil and cytosine supplementation can help against acetic acid toxicity, with thymine having no effect [22], [30]. This points to the possible inhibition of very specific (and complimentary) nucleotide formation from different inhibitor classes.

One approach to dealing with hydrolysate inhibition compounds is using metabolic networks found in other organisms for their complete utilization as a carbon source. Complete metabolism of phenolics by Coniochaeta ligniaria [31] and HMF by Cupriavidis basilensis [32] has been observed. This can be taken advantage of by inserting the 
necessary foreign genes into the biofuel production strain, or by pre-culturing the secondary organism on hydrolysate before it is exposed to the production strain (known as "bioabatement").

\subsection{Genetic Manipulation and Characterization Techniques}

Two main strategies have been employed to improve the performance of microbial strains in industrial bioprocesses: metabolic engineering through direct genetic manipulation, and random mutagenesis. The former is limited by available genetic tools and understanding of the genome of the organism in question, yet is very precise. The latter is limited by the throughput of the screening process. If random mutagenesis is followed by enrichment of improved mutants in a selective media (essentially, evolution), screening can be improved but can only be based on growth rate and reproductive superiority in the selective medium. In either case, understanding the benefits conferred via random mutagenesis is traditionally quite difficult, though recent advances in whole-genome sequencing and transcriptomics have accelerated this process.

\subsubsection{Metabolic Engineering}

Much is known about the metabolism of model organisms like E. coli, and $S$. cerevisiae. The pathways by which compounds are processed for energy or incorporated into biomass, the enzymes and co-factors involved, the molecules produced, and the genetic basis for the regulation of these processes have been studied in depth. For instance, recent metabolic network models for $E$. coli account for the function of 1260 genes and 1039 unique metabolites [33]. In addition, many tools have been developed to efficiently alter the genetic makeup of these organisms, from whole gene deletions and insertions to individual nucleotide alterations. Foreign DNA from other organisms can even be introduced into model organisms' genomes to accomplish non-native functions [34].

Models and methods like these do exist for algae (and plant species in general), but they are not nearly as well developed or widely used. While advancements in genetic sequencing technology are allowing photosynthetic organism genome and metabolic modeling to progress, the techniques for actual genetic manipulation of the organisms are only in the very beginning stages of development [35], [36]. For heterotrophic model organisms, however, the combination of detailed metabolic network understanding and 
advanced genetic manipulation techniques allows for the rational design of substrate utilization and terminal product creation characteristics. For instance, through a combination of foreign organism gene incorporation and native gene overexpression/inactivation, E.coli has been engineered to produce several promising nonnative biofuels, such as isopropanol and n-butanol [37].

\subsubsection{Improvement of Growth and Tolerance Through Evolution}

Natural mutagenesis and selection in challenging growth conditions (adaptive evolution) is a powerful tool for microbial strain improvement, particularly when dealing with a microbial strain with poor genetic manipulation tools available, a selective factor whose mechanisms of inhibition are unknown, or complex growth media where selective pressures themselves are unknown. For instance, wastewater treatment plants utilize a continually evolving consortium of microbes to metabolize an incredibly varied mix of organic and inorganic compounds from multiple industrial and agricultural processes [38].

Single production strains have been evolved for both enhanced substrate consumption and inhibitor tolerance, usually by either continuous cultivation or serial passage of batch cultures to fresh media. E. coli has been evolved to optimally grow on the non-preferred carbon source glycerol [39] and even citrate, a carbon source that is normally completely ignored under oxic conditions [40]. Various strains have also

developed resistance to inhibitor compounds via evolution. S. cerevisiae resistance to phenols was improved via evolution on high-phenolic corncob residues [41]. E. coli has also been evolved for increased tolerance of isobutanol, a promising next-generation biofuel that is considerably toxic at low concentration [42].

\subsubsection{Full Genome Sequencing}

The speed, scope, and cost-efficiency of genetic sequencing technology is improving at a high rate, even exceeding the "Moore's Law" trend of exponential computing power increases in general (Figure 1.2) [43]. Sequencing the entire genome of an organism, even a simple bacterium, was a six-year endeavor two decades ago [44], but current nextgeneration sequencing technology can complete this task in mere days [43]. 


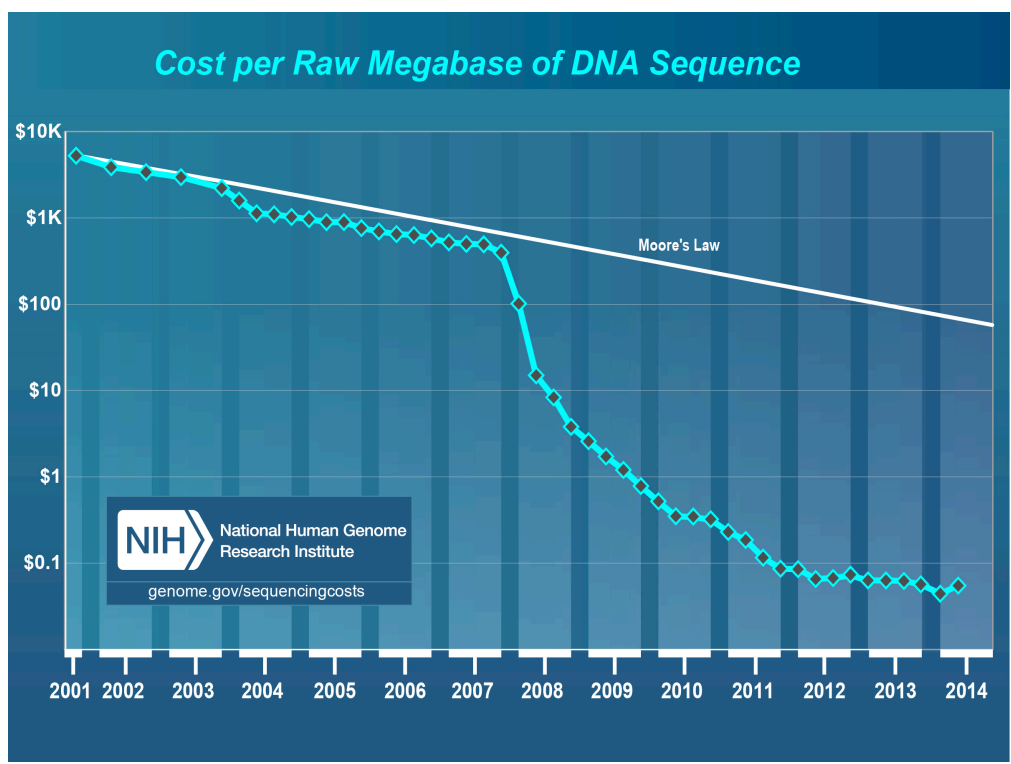

Figure 1.2 The declining cost of sequencing per megabase pair of DNA. [Wetterstrand KA. DNA Sequencing Costs: Data from the NHGRI Genome Sequencing Program (GSP) Available at: www.genome.gov/sequencingcosts. Accessed 2014.]

These advancements have led to the use of full genome sequencing as a tool to determine the full genome of evolved microbial species and compare them to their parent strain to identify the mutations responsible for the improved phenotypes. The previously mentioned projects by Fong et al and Minty et al utilized full genome sequencing to identify the mutations responsible for evolved E. coli improved glycerol metabolism and isobutanol tolerance, respectively, which they then separately reintroduced to a base E. coli strain and studied individually [39], [42].

\subsubsection{Transcriptome Sequencing}

Advances in sequencing technology have also enhanced the speed and specificity of determining gene expression levels by replacing Northern Blot and microarray analyses with the direct sequencing tRNA [45]. This technology is often used to study gene expression levels in human stem cells and cancer cells [46], [47], but has been applied to bacteria as well. A recent study generated complete transcriptome data for a pathogenic strain of $E$. coli grown under a variety of stress conditions. The strain had a poorly annotated genome, and the gene expression results were used to back-calculate which genes were regulated during specific stresses and narrow down possible genes relating to pathogenicity [48]. 


\subsection{Dissertation Overview}

This thesis focuses on the challenge of effective nutrient cycling within an algae biomass-derived biocrude oil process, specifically the utilization of the aqueous phase (AqAl) formed during hydrothermal liquefaction (HTL). Instead of pursuing the conventional method of attempting recycle directly to an algae pond, we investigated the possibility of a bacterial side culture as an intermediate step between HTL and algae growth (Figure 1.3).

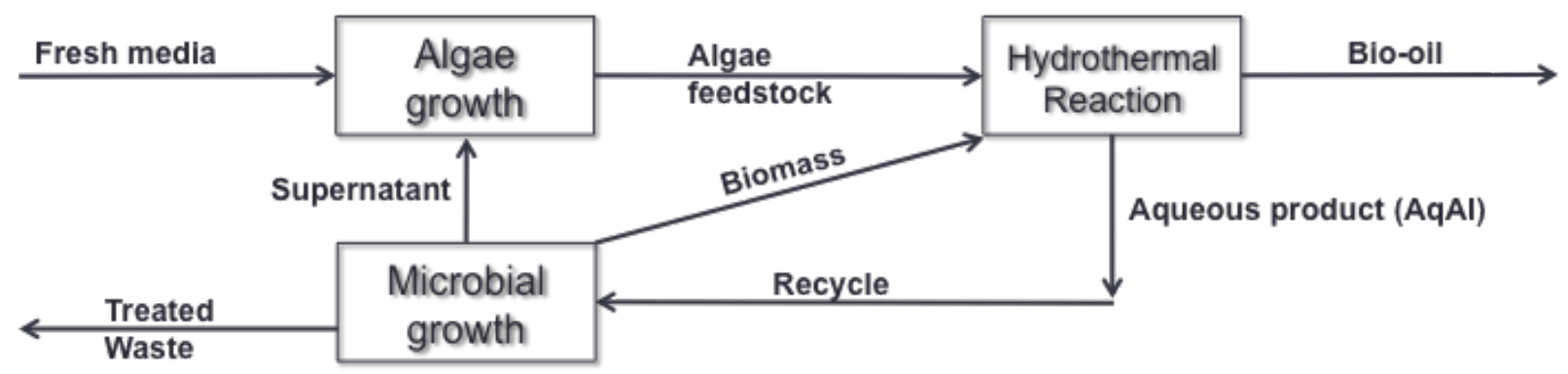

Figure 1.3 Proposed intermediate microbial culture for algae bio-oil production process

The potential benefits of this setup are twofold. First, bacteria could perform a bioabatement role by metabolizing compounds in AqAl inhibitory to algae or remineralizing nutrients tied up in organic compounds, much like bacteria's role in natural symbiotic heterotroph/phototroph systems [49]. Second, the additional microbial biomass generated could supplement algae biomass in the HTL reaction.

Our first goal was to characterize the properties of AqAl formed under various HTL reaction conditions and determine the feasibility of microbial culture on AqAl. Part I begins with a collaborative effort (with the Phillip Savage Lab at University of Michigan) to determine yield and $\mathrm{C} / \mathrm{N} / \mathrm{P}$ fractioning among the products formed from HTL of alga Nannochloropsis oculata under a reaction space of various times and temperatures. We used these results to choose a certain reaction condition that corresponded to good oil yields (350 ${ }^{\circ} \mathrm{C}$ for one hour) and establish base case growth of model bacterial strains Escherichia coli and Pseudomonas putida using the AqAl as the sole C/N/P source. E. coli was chosen due to the large amount of genomic knowledge and available genetic manipulation tools available for it (important for later analyses) and the soil bacterium $P$. putida was chosen due to its diverse metabolic network. 
This proof of concept study showed both microbes could indeed grow using AqAl as the only $\mathrm{C} / \mathrm{N} / \mathrm{P}$ source with only supplementation of minor micronutrients, and at optimal concentrations more than an order of magnitude more than handled by algae (20 vol\% $\mathrm{AqAl}$ as opposed to $0.5 \mathrm{vol} \%$ ). However, organic uptake was poor and was worsened with increasing AqAl concentration, suggesting limited metabolism of certain substrates and toxic effects.

Considering the unknown and likely multiple compounds responsible for these challenges, we opted to attempt improvement of the microbes through adaptive evolution instead of direct metabolic engineering. Part II focuses on an evolution study where E. coli and $P$. putida were grown over $\sim 300$ generations via serial transfer of cultures into media containing an increasing concentration of AqAl. This was sufficient time to generate evolved strains capable of growing faster and to higher yields than their parents.

The full genomes of 18 improved strains were sequenced and acquired single nucleotide polymorphism mutations (SNPs) were identified. Several of these SNPs were individually added to a lab strain of $E$. coli for further analysis of their specific benefits to the organism and possible mechanisms of action. Also, the top-performing evolved E. coli strain, along with its parent strain, were grown in both ideal minimal medium and AqAl medium, RNA samples were extracted, and full transcriptomes were sequenced in order to quantify differential gene expression between parent and mutant in the two media.

The evolution study produced immediately useful bacterial strains for the processing of AqAl (assuming similar algae strains and reaction conditions are used). But, through genomic sequencing, individual mutation investigation, and transcriptomic study, it also produced a better understanding of what the growth inhibiting properties of AqAl are, how bacterial cells react to them, and possible methods of alleviating them.

Part III focuses on the proposed two-fold usefulness of the microbial preculture. It begins with another collaborative work (also with the Savage Group) concerning the hydrothermal liquefaction of microbial biomass. In addition to the E. coli and P. putida strains already being studied, Gram-positive Bacillus subtilis and yeast Saccharomyces cerevisiae were studied to diversify the microbial feedstock pool and represent possible 
biomass sources from other industrial processes. We found that the whole-cell conversion advantage that HTL has for various algae species holds true for microbial strains as well, with biocrude oil yield similar to that of Nannochloropsis for all microbial strains except $B$. subtilis.

Part III concludes with preliminary results from a study determining the effects microbial pretreatment has on AqAl before it is recycled for algae growth. AqAl was generated and used to make bacterial growth media. We grew E. coli and P. Putida in these media, filtered the cells out, then used the filtrate to formulate algae growth media. We grew alga Chlorella vulgaris in these media and compared results to growth data from "unprocessed" AqAl, and the microbial pretreatment does indeed appear to have a positive effect on algae growth at highly inhibitive concentrations of AqAl. 


\title{
Part I
}

\section{Determining the properties of Nannochloropsis HTL products and culturability of model microbes on the aqueous product}

\author{
Chapter 2 \\ Hydrothermal Liquefaction of Nannochloropsis sp.: Systematic study of process \\ variables and analysis of the product fractions
}

\subsection{Summary}

We investigated hydrothermal liquefaction of Nannochloropsis sp. at different temperatures $\left(250-400{ }^{\circ} \mathrm{C}\right)$, times $(10-90 \mathrm{~min})$, water densities $(0.3-0.5 \mathrm{~g} / \mathrm{mL})$, and biomass loadings (5 - 35 wt \%). Liquefaction produced a biocrude with light and heavy fractions, along with gaseous, aqueous, and solid by-product fractions. The gravimetric yields of the product fractions from experiments at $250{ }^{\circ} \mathrm{C}$ summed to an average of $100 \pm$ 4 wt \%, showing mass balance closure at $250{ }^{\circ} \mathrm{C}$. The gravimetric yields of the product fractions are independent of water density at $400{ }^{\circ} \mathrm{C}$. Increasing the biomass loading increases the biocrude yield from 36 to $46 \mathrm{wt} \%$. The yields of light and heavy biocrude depend on reaction time and temperature, but their combined yield depends primarily on temperature. Regardless of reaction time and temperature, the yield of products distributed to the aqueous phase is $51 \pm 5 \mathrm{wt} \%$ and the light biocrude is $75 \pm 1 \mathrm{wt} \% \mathrm{C}$. Two-thirds of the $\mathrm{N}$ in the alga is immediately distributed to the aqueous phase and up to $84 \%$ can be partitioned there. Up to $85 \%$ of the $\mathrm{P}$ is distributed to the aqueous phase in the form of free phosphate. Thus, $\mathrm{N}$ and $\mathrm{P}$ can be recovered in the aqueous phase for nutrient recycling. Experiments at $350{ }^{\circ} \mathrm{C}$ for $60 \mathrm{~min}$ resulted in $26 \%$ of the $\mathrm{P}$ distributing to the biocrude. Up to $80 \%$ of the chemical energy in the alga is retained within the biocrude. The quantitative results reported herein provide the basis for a reaction network for algae liquefaction. 
The entirety of the material presented in this section has been published in Biomass and Bioenergy as follows: Peter J. Valdez, Michael C. Nelson, Henry Y. Wang, Xiaoxia Nina Lin, and Phillip E. Savage. 2012. "Hydrothermal liquefaction of Nannochloropsis sp.: Systematic study of process variables and analysis of the product fractions." Biomass and Bioenergy, 46, 317-331.

Michael Nelson, the $2^{\text {nd }}$ author and preparer of this dissertation, was responsible for experimentation and written material concerning the aqueous phase of the reactions studied. Peter Valdez, the $1^{\text {st }}$ author, was responsible for experimentation and written material concerning hydrothermal liquefaction reactions, product extractions, and solid/liquid/oil analyses. The material is being used with permission of the authors.

\subsection{Introduction}

Hydrothermal liquefaction is an emergent technology for converting wet algal biomass into an energy-dense biocrude oil. Liquefaction of the cellular material occurs as the biomacromolecules react in and with water at elevated temperatures (e.g., $350{ }^{\circ} \mathrm{C}$ ) and pressures to form smaller molecules that are closer to being in the range required for liquid fuels. Microalgae have production yields higher than terrestrial oilseeds commonly used as biofuel feedstocks [50] and can capture $\mathrm{CO}_{2}$ emissions [51] or treat wastewater effluent during cultivation [52]. Liquefaction of wet or aquatic biomass obviates energy and resource-intensive steps, such as drying and solvent extraction, that are common for other biofuel conversion processes such as pyrolysis and transesterification. Microalgal liquefaction produces high yields of biocrude, with the highest reported yield of 64 wt \% [53]. The biocrude and gas products from liquefaction capture as much as $90 \%$ of the energy content of the microalgal feedstock [18].

Recent studies of microalgal liquefaction have examined processing parameters such as residence time [10], [54]-[56], reaction temperature [10], [18], [53]-[56], catalyst selection [7], [19], [53], [55]-[57], feedstock selection [7], [19], and biomass loading [10]. These results from the literature provide some clues about the reaction pathways, but not enough information to develop an entire reaction network. As the field progresses, quantitative reaction models based on the governing reaction network will be needed for 
process design and optimization. Developing such a model requires data from a systematic study of the influence of all the relevant process variables on liquefaction of a single alga.

To date, there have only been a few studies that examined how residence time affects the yields and elemental composition of the liquefaction products [10], [54]-[56]. Jena et al. examined four holding times, but only for a single temperature $\left(350^{\circ} \mathrm{C}\right)$ and in an autoclave reactor (1.8 L) [10]. Similar experiments were conducted in large autoclave reactors ( $>100$ $\mathrm{mL}$ ) at holding times of 5, 30 and $60 \mathrm{~min}$ and at nominal temperatures of 250, 300, and 340 ${ }^{\circ} \mathrm{C}$ [55], [56]. Garcia Alba et al. used a smaller batch reactor ( $45 \mathrm{~mL}$ ) to achieve heat-up times of 6-7 min. They examined holding times of 5, 15, 30, $60 \mathrm{~min}$, but for only 2 temperatures $\left(200,300{ }^{\circ} \mathrm{C}\right)$ [54]. It is not clear that the data from these studies can be treated as being from isothermal reactions because of the large reactor volumes and correspondingly large thermal mass and slow heat-up times. Thus their use in kinetic modeling is limited.

Directly accounting for all of the mass initially in the algae is another important step for developing an accurate model of algal liquefaction. Previous work showed that the difficulty in closing the mass balance arises from the difficulty in directly obtaining a gravimetric yield of the liquefaction products that partition into the aqueous phase. Most researchers have simply assumed closure of the mass balance and determined the yield of the aqueous-phase products as the difference between the total biomass loaded into the reactor and the total mass collected from the biocrude, gas, and solid products [7], [10], [19], [58].

To date, the effect of water density at supercritical conditions on the product fractions from the hydrothermal liquefaction of microalgae has been unexplored. Brown et al., Jena et al., and Garcia Alba et al. studied liquefaction of microalgae at supercritical conditions $\left(>221 \mathrm{bar}, 374^{\circ} \mathrm{C}\right.$ ) but did not examine the effect of water density at a fixed temperature [10], [18], [58].

The effect of biomass loading has not been thoroughly addressed. Jena et al. examined biomass concentrations of $10-50 \mathrm{wt} \%$ of Spirulina slurry. Although they found a minimal effect of the loading on the biocrude yield, it is not clear if their results can be extrapolated 
to other species of microalgae. The biomass to water ratio for liquefaction reactions can have a significant impact on the economics of this process and merits further examination [59].

Given these gaps in the literature, we initiated an experimental program of algal liquefaction that used mini-batch reactors with a relatively fast heat-up time of $<3 \mathrm{~min}$. We report herein the results of a comprehensive study of the effects of time, temperature, biomass loading, and water density on the yields and elemental composition of the product fractions. We use these data to propose the basic structure for the reaction network for the hydrothermal liquefaction of microalgae. We attempted to directly measure the yield of products in the aqueous phase instead of relying solely on calculating this value by difference. We also provide the most detailed characterization to date for the different forms of carbon, nitrogen, and phosphorus in the aqueous phase products.

\subsection{Overview}

We performed HTL reactions on slurries of Nannochloropsis biomass at temperatures of $200,250,350$, and $400{ }^{\circ} \mathrm{C}$ for 10 to 90 minutes. We also included "zero minute" reactions where loaded reactors were not heated, but still processed with the same product extraction method. Figure 2.1 illustrates our experimental procedure for separating gas, solid, aqueous, and biocrude oil products. The biocrude oil was separated into light and heavy fractions based on n-hexane solubility. Products were subjected to various analyses, including mass yield, energy yield, and elemental composition.

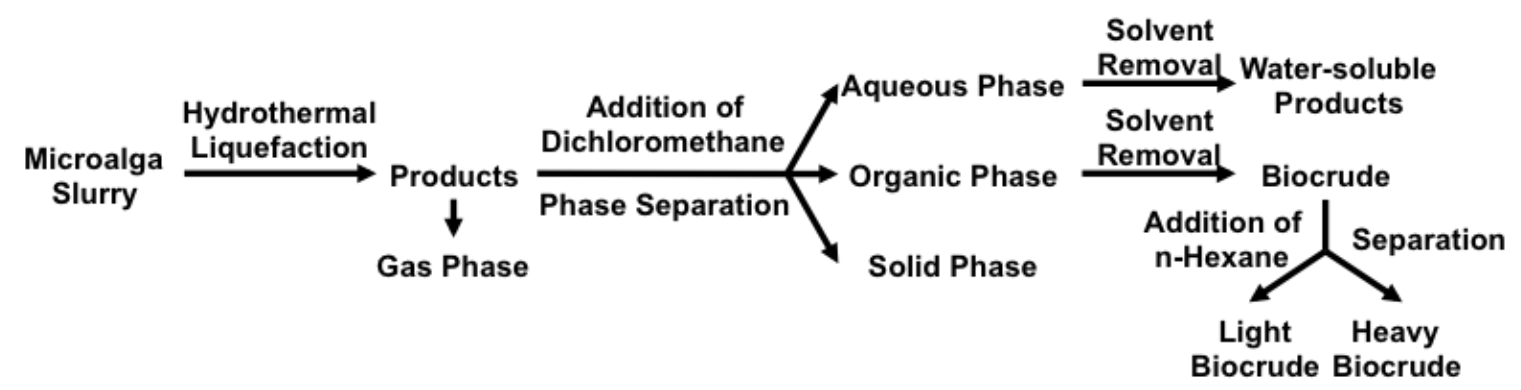

Figure 2.1 Procedure for reaction and product work-up

\subsection{Results and Discussion}

This section reports the four metrics of yield, elemental composition, elemental distribution, and energy distribution, which we quantified for each of the product fractions. 
We first discuss how time, temperature, and wt \% loading affect the metrics for each of the liquefaction product fractions. The values for the four metrics from experiments at different water densities, but otherwise identical conditions, were so close to one another that the small differences are very likely within the experimental error. Therefore, we report only the results from one water density $(0.5 \mathrm{~g} / \mathrm{mL})$ to compare results from supercritical and subcritical conditions. Yields of the product fractions at lower water density values are available in the supplemental information.

The present experiments were not replicated, except for one experiment at $350{ }^{\circ} \mathrm{C}$ for 60 min to demonstrate run-to-run variation, although past research in our lab has shown that the variation in gravimetric yields was typically $3-8 \%$ on a relative basis [18], [20]. Replicates from the experiment at $350{ }^{\circ} \mathrm{C}$ for 60 min verified that run-to-run variation of the yields of aqueous-phase products, biocrude, and solids were 5,6 , and $12 \%$ on a relative basis, respectively. The variation from the analytical results from chemical measurements of the aqueous phase with the exception of total $\mathrm{N}$ were $<10 \%$ on a relative basis. Measurements of total $\mathrm{N}$ in the aqueous phase varied by $16 \%$ on a relative basis. When applicable, we report the mean and the uncertainties reported in tables and figures are one standard deviation.

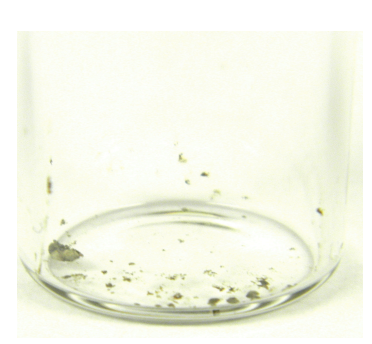

(a)

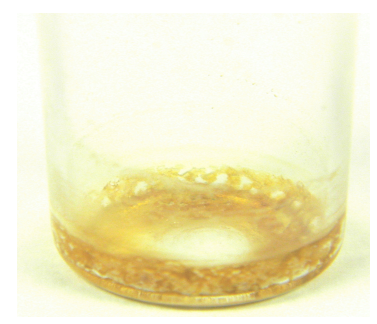

(b)

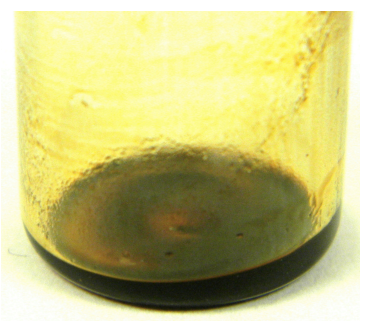

(c)

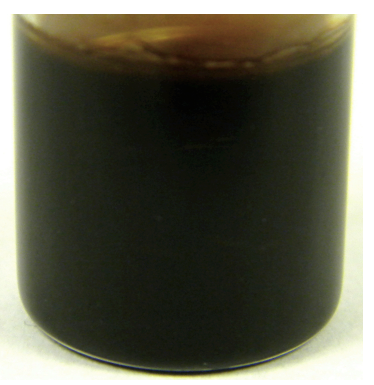

(d)

Figure 2.2 Liquefaction product fractions: a) solids, b) water-soluble products, c) light biocrude, and d) heavy biocrude produced at $350^{\circ} \mathrm{C}$ for $60 \mathrm{~min}$ 


\subsubsection{Product Yields}

Table 2.1 shows the yield of each product fraction obtained from the original algal biomass. The yields varied at the different reaction temperatures, presumably because modestly different amounts of slurry were used to load reactors. The results show that some biocrude and aqueous-phase products can be obtained from the algae simply by extraction using the outlined procedure. Of course much higher biocrude yields are available through liquefaction.

Table 2.1 Average initial content of product fractions in the feedstock

\begin{tabular}{cc}
\hline product fraction & initial content (wt \\
$\%$ ) \\
gas & 0 \\
light biocrude & $6.5 \pm 1.8$ \\
heavy biocrude & $2.7 \pm 1.5$ \\
solids & $68 \pm 5$ \\
water-soluble & $21 \pm 2$ \\
products & \\
\hline total & $98 \pm 6$ \\
\hline
\end{tabular}

We collected and directly measured the yields of the solids, light and heavy biocrudes, and water-soluble products. Figure 2.2a shows that the solids appear as a gray powder. At lower reaction temperatures or times the solids showed more green hues suggesting compounds such as chlorophyll from the cells may be present and intact in the solids. The aqueous phase was always amber in color with a strong odor of ammonia. The amber color intensified as time and temperature increased and the solution was always semitransparent. Figure $2.2 \mathrm{~b}$ shows that when the aqueous phase was dried, the water-soluble products maintained a similar amber color. The water-soluble products were malleable 
and soft with a similar foul odor. Both biocrudes (light and heavy) were dark-brown as shown in Figure 2.2c and Figure 2.2d, respectively. The volume of heavy biocrude cannot be inferred from the photograph since the highly viscous product adheres to the walls of the vial when dried.

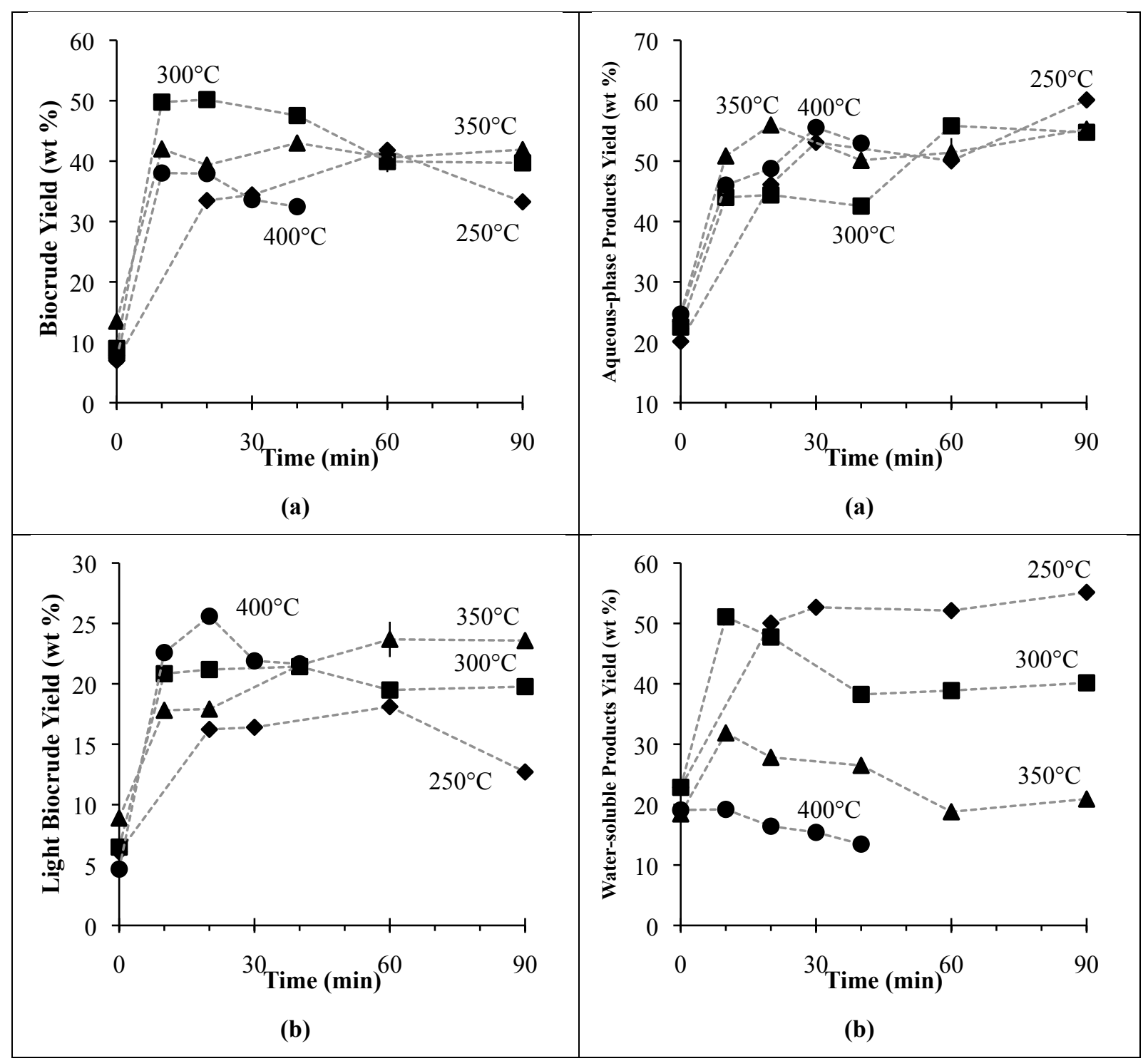




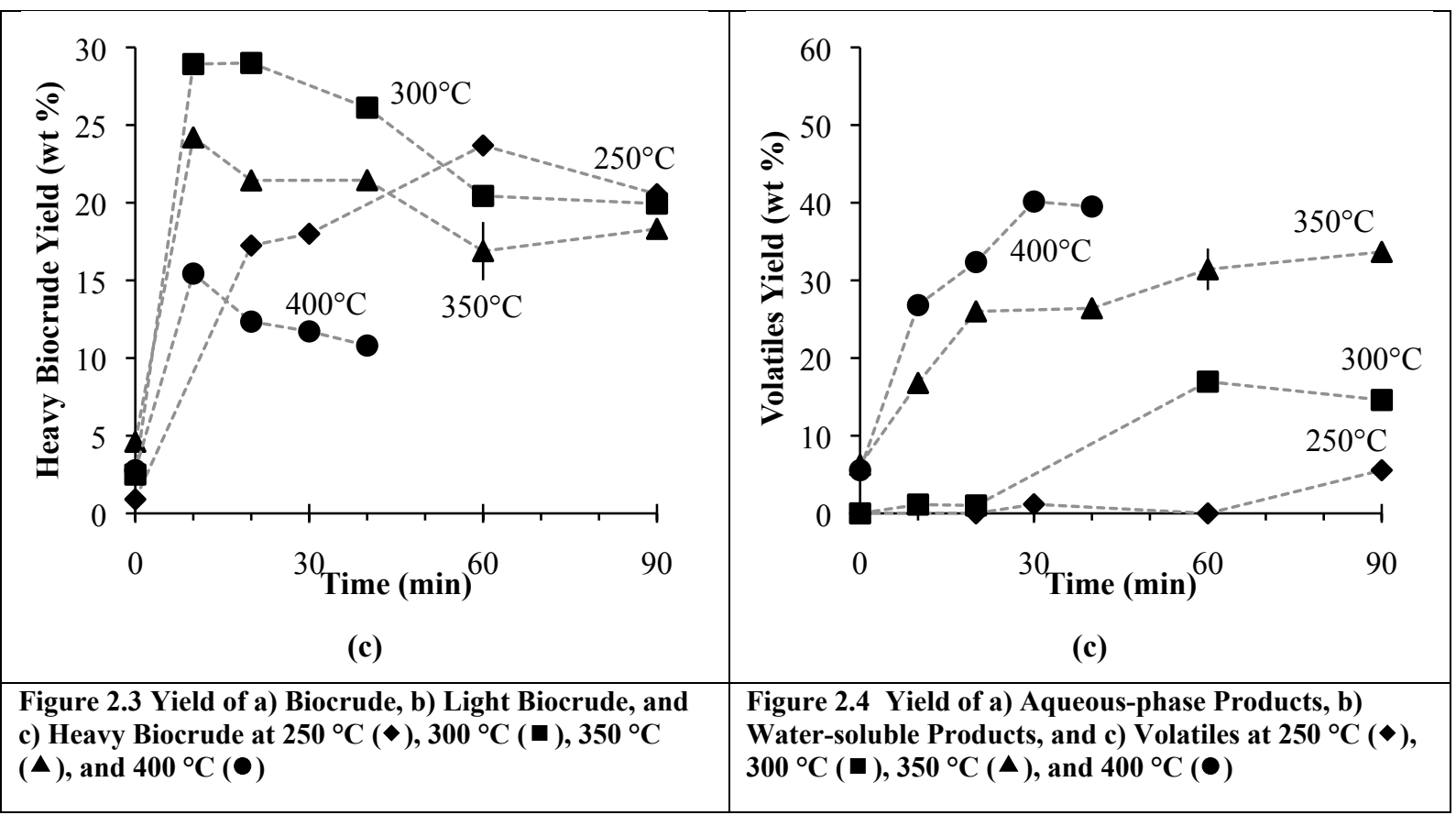

The total yield of biocrude (light plus heavy), illustrated in Figure 2.3a, is between 40 $50 \%$ at 300 and $350{ }^{\circ} \mathrm{C}$. Although some biocrude is lost during drying, it is generally $<3 \%$ of the total mass of the biocrude [20]. There is little variation in the biocrude yield with time at $350{ }^{\circ} \mathrm{C}$. The yields at 250 and $400{ }^{\circ} \mathrm{C}$ were almost always lower than the yields at 300 and $350{ }^{\circ} \mathrm{C}$, which is consistent with previous work with this alga [18]. Above the critical point of water there is a decrease in the overall yield of biocrude at longer times, perhaps because some of the biocrude is thermally converted to smaller gaseous molecules.

Figure 2.3b shows that the yield of light biocrude at a fixed time often increases with temperature. These results are consistent with the trend suggested by Jena et al. [10]. The highest yield of light biocrude is 25 wt $\%$ at $400{ }^{\circ} \mathrm{C}$ and $20 \mathrm{~min}$. The yield then drops as time progresses. At $300{ }^{\circ} \mathrm{C}$ the light biocrude yield is fairly constant with time variations. The yield of light crude produced at $350{ }^{\circ} \mathrm{C}$ shows a steady increase with increasing time. The yield is lowest at $250{ }^{\circ} \mathrm{C}$. Analysis of the light biocrude via gas chromatography showed the presence of fatty acids, chlorophyll derivatives, and cholesterol derivatives, as had been reported from previous analyses of biocrude from this alga [18], [20]. 
Figure 2.3c shows that the yield of heavy biocrude is initially higher than that of the light biocrude at 300 and $350{ }^{\circ} \mathrm{C}$. Above $300{ }^{\circ} \mathrm{C}$, increasing temperature produces less heavy biocrude and the yield of heavy crude decreases as time increases. The initial increase in the yield of heavy biocrude at $250{ }^{\circ} \mathrm{C}$ and its subsequent decrease at the more severe reaction conditions may discount the previous assumption of free radical polymerization forming large amounts of high molecular weight compounds during liquefaction [54], [60]. Polymerization may still take place, but it may not be a dominant reaction, allowing for the conversion of other heavy biocrude components into gas, light biocrude, and aqueous-phase products.

There appears to be some interconversion of material in the light and heavy biocrude fractions as the reaction progresses. At $350{ }^{\circ} \mathrm{C}$ and $20 \mathrm{~min}$ and at more severe conditions, the yield of light biocrude surpasses the yield of heavy biocrude. The increasing yield of the light biocrude, coupled with the concomitant decrease of heavy biocrude, suggests the transformation of heavy biocrude compounds into light biocrude compounds.

Figure 2.4a shows the total yield of products distributed to the aqueous phase. This yield is determined as the difference between $100 \mathrm{wt} \%$ and the sum of the yields of the other product fractions (biocrude, gas, and solids). About $51 \pm 5 \mathrm{wt} \%$ of the initial mass of algae resides in the aqueous phase after the reaction. The change in yield of products distributed to the aqueous phase is almost entirely independent of the liquefaction temperature. As time increases, so does the yield of the aqueous phase products, but only slightly. These results suggest that the products in the aqueous phase primarily come directly from the algal biomass and that compounds originally partitioned into the biocrude fraction that get converted into aqueous-phase products account for only a small amount of the material.

Figure $2.4 \mathrm{~b}$ illustrates that the yield of the water-soluble products quickly increases to about 50 wt $\%$ at both 250 and $300^{\circ} \mathrm{C}$. In fact, the yields of water-soluble products at 250 ${ }^{\circ} \mathrm{C}$, which were determined directly, are on average within $1 \mathrm{wt} \%$ of the yield of aqueousphase products in Figure 2.4a, which was determined by difference. These yields being essentially equal at the mild liquefaction conditions lends credence to calculating the total 
yield of aqueous-phase products by difference. At $250{ }^{\circ} \mathrm{C}$ we accounted for, on average 100 \pm 4 wt $\%$ of the initial biomass in all of the products. The yield of water-soluble products in Figure $2.4 \mathrm{~b}$ decreases with time at 300,350 , and $400^{\circ} \mathrm{C}$. Presumably, the compounds in the aqueous phase decompose into highly volatile constituents that are not recovered [7]. We determined the yield of these volatiles formed in the aqueous phase as the difference between $100 \mathrm{wt} \%$ and the sum of the yields of the other product fractions (biocrude, gas, solids, and water-soluble products).

Figure 2.4c shows that the yield of the volatiles is nearly the mirror image of the yield of the water-soluble products. The yield of volatiles increases steadily with both time and temperature. These trends are consistent with the hypothesis that the volatiles, which are lost during the drying of the aqueous phase, come from the decomposition of compounds originally partitioned into the aqueous phase very early in the reaction. Thus, there appear to be significant reactions taking place within the aqueous phase itself, wherein large compounds with low vapor pressure are converted into lighter products.

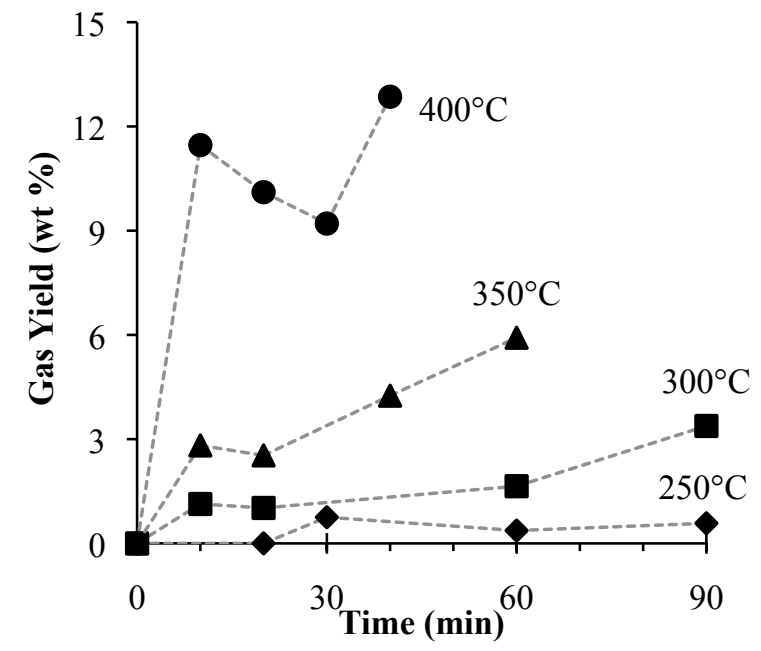

(a)

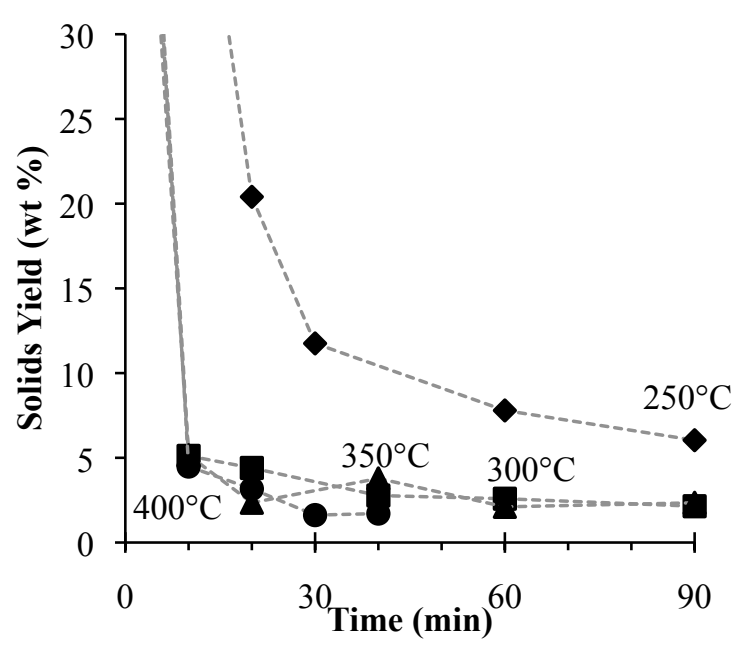

(b)

Figure 2.5 Yield of a) Gas and b) Solids at $250^{\circ} \mathrm{C}(\bullet), 300^{\circ} \mathrm{C}(\square), 350^{\circ} \mathrm{C}(\Delta)$, and $400{ }^{\circ} \mathrm{C}(\bullet)$

Figure 2.5a illustrates that the yield of gases $\left(\mathrm{H}_{2}, \mathrm{CO}, \mathrm{CH}_{4}, \mathrm{CO}_{2}, \mathrm{C}_{2} \mathrm{H}_{4}, \mathrm{C}_{2} \mathrm{H}_{6}\right)$ increases as both time and temperature increase. At $250{ }^{\circ} \mathrm{C},<1 \mathrm{wt} \%$ of the algae is converted into gas, but the yield reaches $13 \mathrm{wt} \%$ at $400{ }^{\circ} \mathrm{C}$. Similar to previous results, at least $77 \mathrm{~mol} \%$ of the product gases is composed of $\mathrm{CO}_{2}$ at supercritical conditions [18], [20]. At subcritical 
conditions, $>93 \mathrm{~mol} \%$ of the gas product is $\mathrm{CO}_{2}$. Although there is some value in flammable gases such as $\mathrm{H}_{2}, \mathrm{CH}_{4}, \mathrm{C}_{2} \mathrm{H}_{4}$, and $\mathrm{C}_{2} \mathrm{H}_{6}$ they are in small concentrations of $<14 \mathrm{~mol} \%$ combined at $400{ }^{\circ} \mathrm{C}$, and $<7 \mathrm{~mol} \%$ at subcritical conditions.

The initial algal biomass is composed primarily of solid material that is insoluble in both water and dichloromethane. Figure $2.5 \mathrm{~b}$ shows that as time progresses, more of the solid material is transformed into the other product fractions. The yield of solids decreases rapidly at short reaction times and the rate of this initial decrease increases with temperature. At $250{ }^{\circ} \mathrm{C}$ the yield of solids drops to $<8 \mathrm{wt} \%$ after $60 \mathrm{~min}$ of reaction time. The yield of solids is just $3 \mathrm{wt} \%$ after $60 \mathrm{~min}$ of treatment at higher temperatures. Note that there is no region where the yield of solids increases with time and temperature. This result suggests that over-reaction of the biocrude to produce large dichloromethaneinsoluble compounds does not occur to any appreciable extent at the conditions investigated. After a short period of hydrothermal treatment, it is likely that the intracellular contents of the alga are released so that there is a sharp decrease in yield of solids and sharp increase in the yields of water-soluble products and light and heavy biocrude fractions.

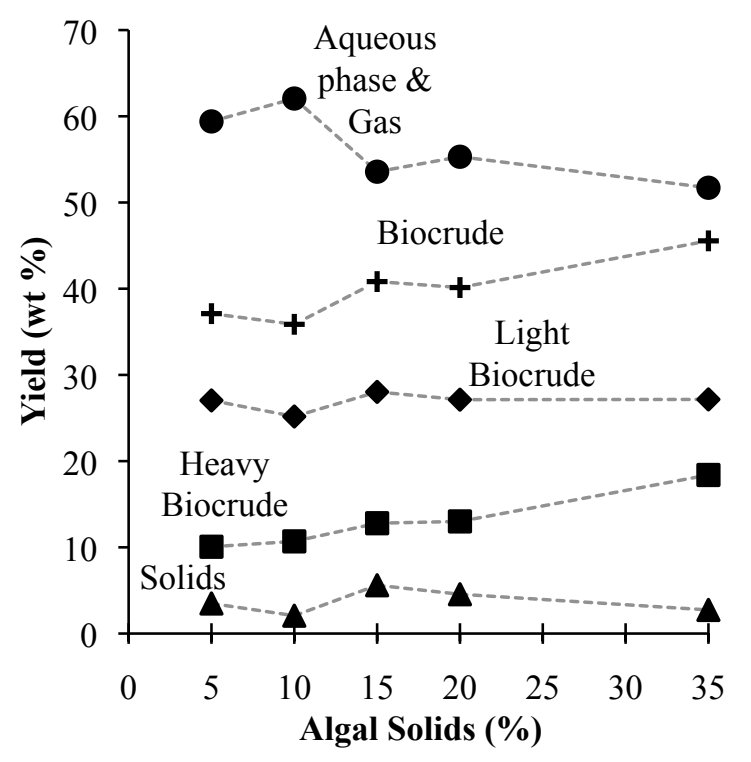

Figure 2.6 Yields of biocrude $(+)$, light biocrude $(\bullet)$, heavy biocrude $(\bullet)$, aqueous-phase products and gas $(\bullet)$, and solids $(\Delta)$ produced at $350{ }^{\circ} \mathrm{C}$ for $60 \mathrm{~min}$ 
Figure 2.6 depicts the change in yields of the liquefaction product fractions with respect to variation of the loading of algal solids. At $350^{\circ} \mathrm{C}$ for $60 \mathrm{~min}$, the biocrude yield increases from $36-46 \mathrm{wt} \%$ as the algae concentration in the slurry increases from 5 - $35 \mathrm{wt} \%$. These results differ from those of Jena et al., which showed no significant variation in the biocrude yield when biomass loading was varied from 10 - 50 wt \% concentrations of Spirulina in water [10]. Thus, it seems that different species of algae might behave differently during hydrothermal liquefaction. The increase in the combined yields of biocrude as the loading increased is due to the increase in the yield of heavy biocrude. The yield of light biocrude remains relatively constant in Figure 2.6. Although higher loadings resulted in higher biocrude yields, Peterson et al. suggest that for hydrothermal processes to be energy and economically efficient, the target biomass loading is 15 - 20 wt \% [59]. The yields of aqueous-phase products and gas are determined by difference and decrease with increased loading. The yield of solids remains relatively unchanged at $4 \pm 1 \mathrm{wt} \%$, as loading increased.

\subsubsection{Elemental Composition}

The alga was $51 \mathrm{wt} \% \mathrm{C}, 7 \mathrm{wt} \% \mathrm{H}, 9 \mathrm{wt} \% \mathrm{~N}, 0.6 \mathrm{wt} \% \mathrm{~S}, 0.6 \mathrm{wt} \% \mathrm{P}$ and $28.8 \mathrm{wt} \% 0$ (by difference). The ash content of the alga was $3 \mathrm{wt} \%$. Qualitative analysis using inductively coupled plasma optical emission spectroscopy (ICP-OES) showed traces of $\mathrm{Co}, \mathrm{Mn}, \mathrm{Fe}, \mathrm{Cu}$, $\mathrm{Zn}, \mathrm{Mo}, \mathrm{Cr}, \mathrm{Mg}, \mathrm{Al}, \mathrm{Na}, \mathrm{K}, \mathrm{Ca}$, and $\mathrm{Cl}$ present in the alga. This section provides information about the elemental composition of the product fractions formed at different liquefaction times and temperatures. 


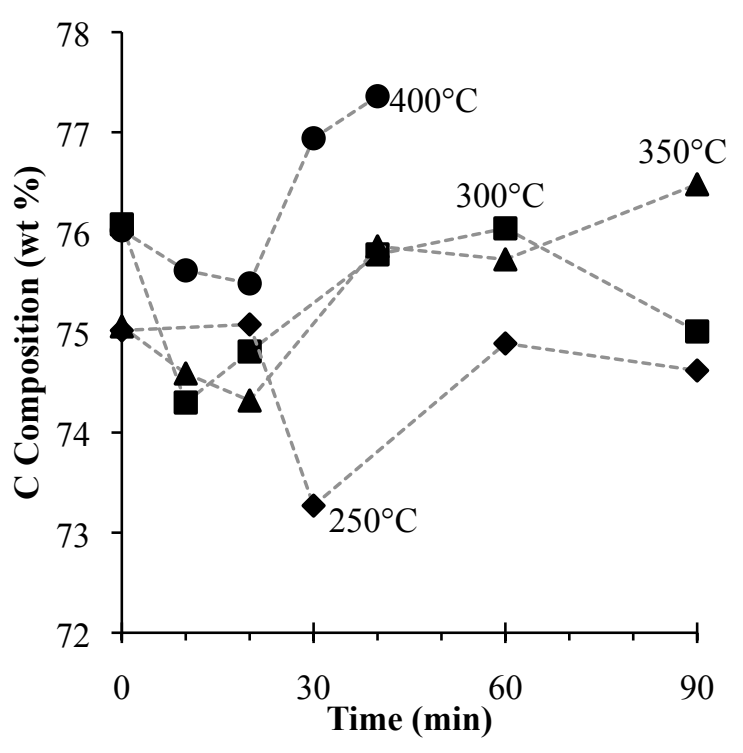

(a)

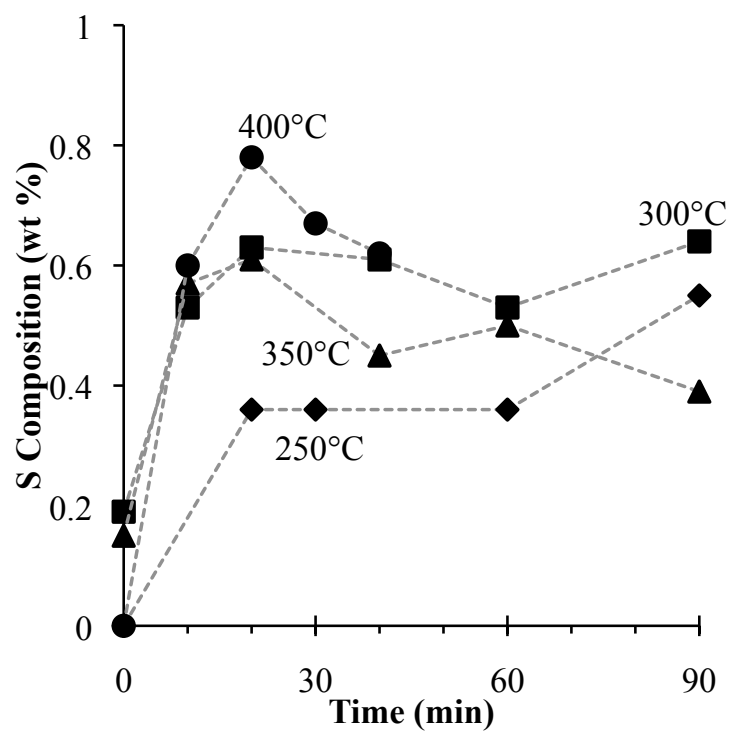

(c)

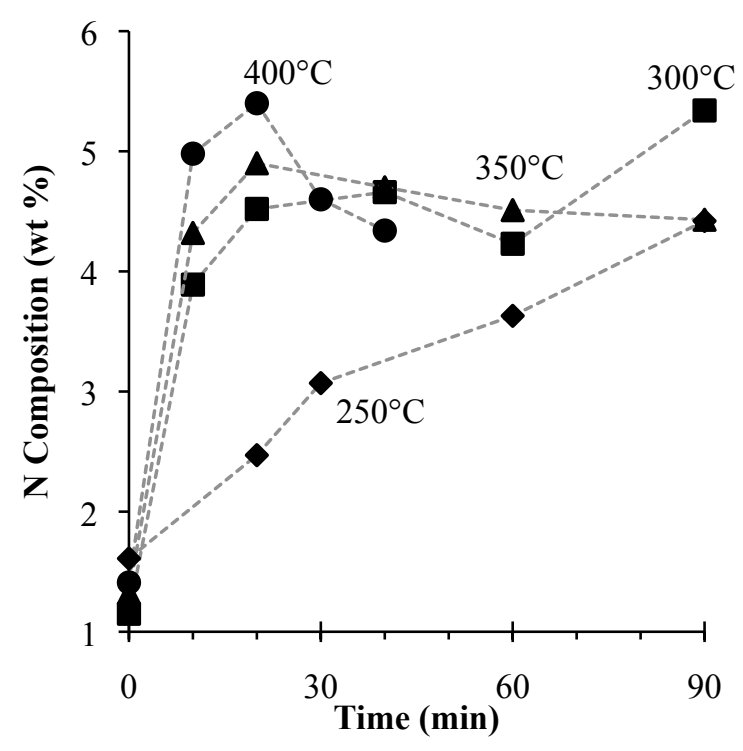

(b)

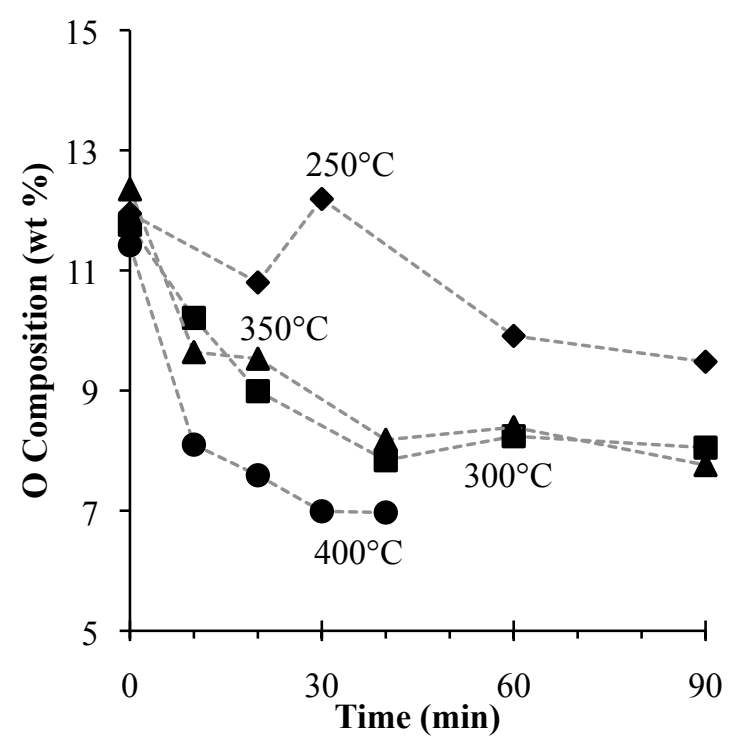

(d)

Figure 2.7 Elemental composition a) $\mathrm{C}$, b) $\mathrm{N}, \mathrm{c}) \mathrm{S}$, and d) $\mathrm{O}$ in the light biocrude at $250{ }^{\circ} \mathrm{C}(\bullet), 300{ }^{\circ} \mathrm{C}(\boldsymbol{\square}), 350^{\circ} \mathrm{C}(\Delta)$, and $400{ }^{\circ} \mathrm{C}(\bullet)$

Figure 2.7 shows the variation of the wt \% of $\mathrm{C}, \mathrm{N}, \mathrm{S}$, and $\mathrm{O}$ in the light biocrude with time and temperature. The $\mathrm{H}$ content of the light biocrude $(10.4 \pm 0.4 \mathrm{wt} \%)$ is independent of both time and temperature and hence not presented here. As shown in Figure $2.7 \mathrm{a}$, the $\mathrm{C}$ composition of the light biocrude varies slightly from as low as 73 wt \% 
at $250{ }^{\circ} \mathrm{C}$ to as high as $77 \mathrm{wt} \%$ at $400{ }^{\circ} \mathrm{C}$, but there is no clear trend with respect to time. The C wt \% generally being $75 \pm 1$ wt \% is consistent with fatty acids and other hydrocarbons being dominant in the biocrude [20]. Palmitic acid, a major component of biocrude from this alga, is $75 \mathrm{wt} \% \mathrm{C}$ which is similar to the average $\mathrm{C}$ composition of the light biocrude [18], [20].

Figure $2.7 \mathrm{~b}$ shows that the $\mathrm{N}$ composition is both time and temperature dependent. The $\mathrm{N}$ composition is initially low, around $2 \mathrm{wt} \%$, but increases to approximately $5 \mathrm{wt} \%$. Increasing temperature increases the rate at which the $\mathrm{N}$ composition reaches this $5 \mathrm{wt} \%$ value. Garcia Alba et al. documented a similar trend in the $\mathrm{N}$ content of biocrude produced at $300{ }^{\circ} \mathrm{C}$ [58]. The increase of the $\mathrm{N}$ content in the biocrude is undesired, reaffirming the need to develop methods to remove the $\mathrm{N}$ from the light biocrude. Many pyrroles and indoles are common in biocrudes and likely contribute to the $\mathrm{N}$ content of the light biocrude since they are difficult to decompose at these conditions [7], [20].

Figure $2.7 \mathrm{c}$ shows that there is some variation in the $\mathrm{S}$ content with time and temperature. The $\mathrm{S}$ content is highest at $400{ }^{\circ} \mathrm{C}$ and lowest at $250{ }^{\circ} \mathrm{C}$. Even so, the $\mathrm{S}$ content is relatively low $(<0.8 \mathrm{wt} \%)$ and not detectable initially. Sulfur in the light biocrude is likely from dimethyl disulfide, which accounts for the majority of the sulfur found in the microalgae [20].

Figure $2.7 \mathrm{~d}$ shows that the 0 content in the light biocrude decreases as time and temperature increase. The terminal value of the wt $\%$ of 0 is strongly dependent on the temperature, ranging from approximately 9 wt $\%$ at $250{ }^{\circ} \mathrm{C}$ to $7 \mathrm{wt} \%$ at $400{ }^{\circ} \mathrm{C}$. Reducing the 0 content in the biocrude is important because doing so increases its energy content. 


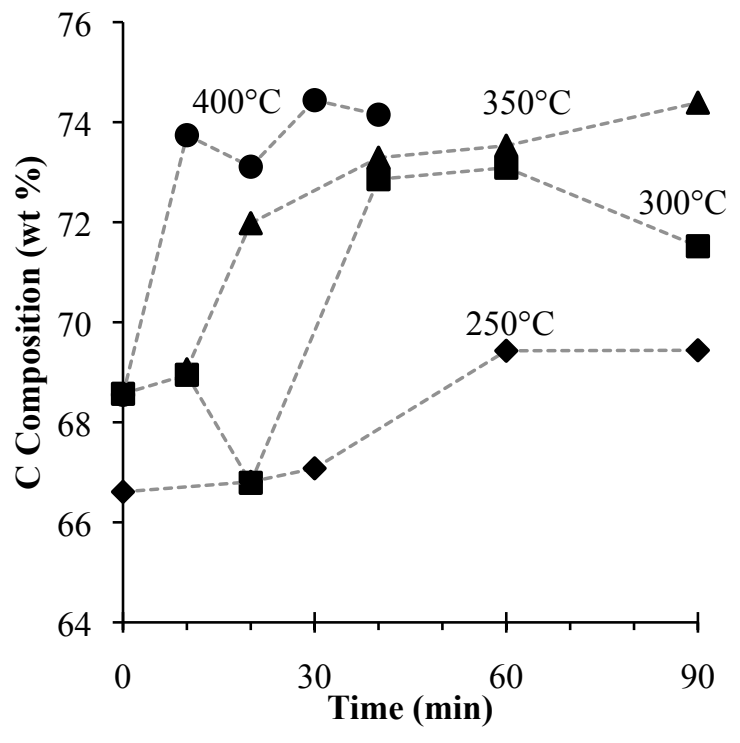

(a)

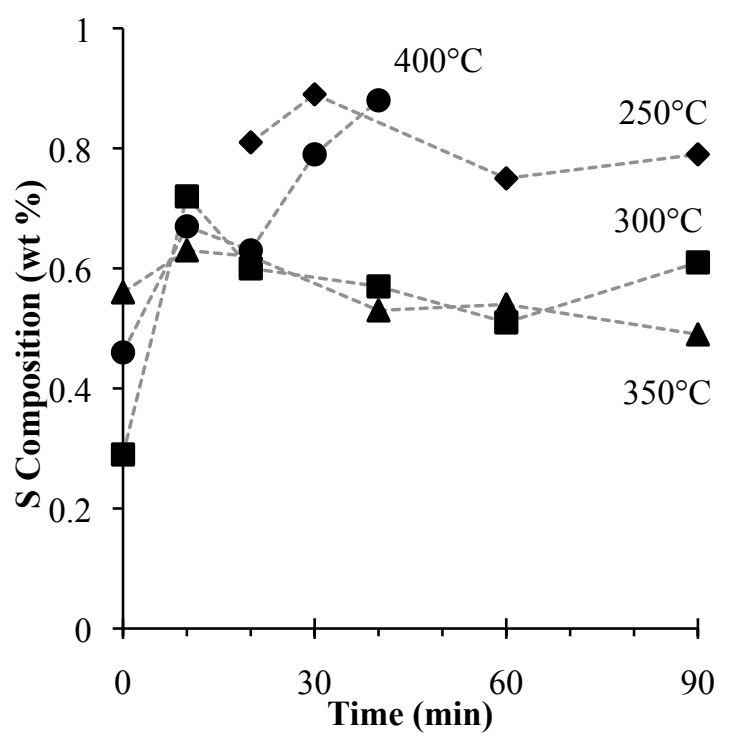

(c)

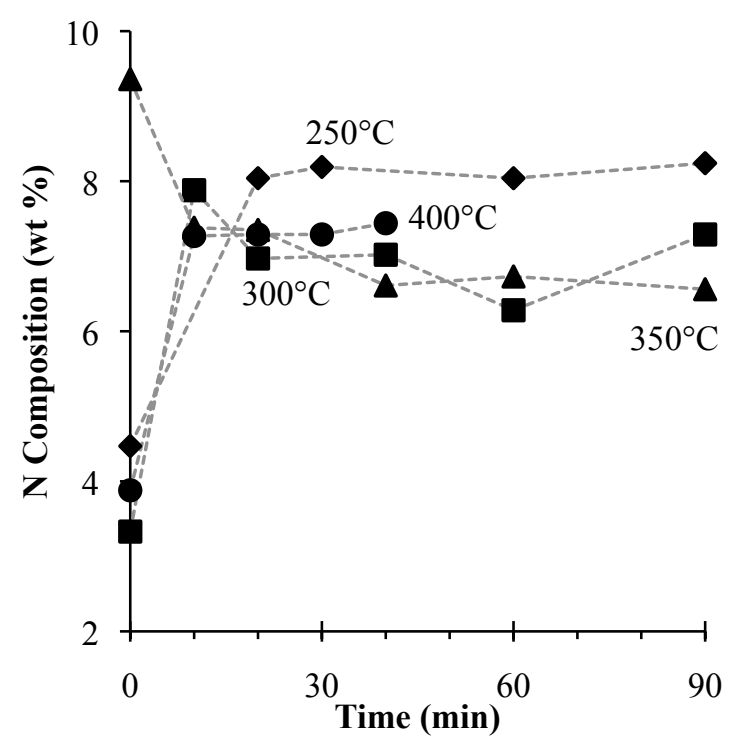

(b)

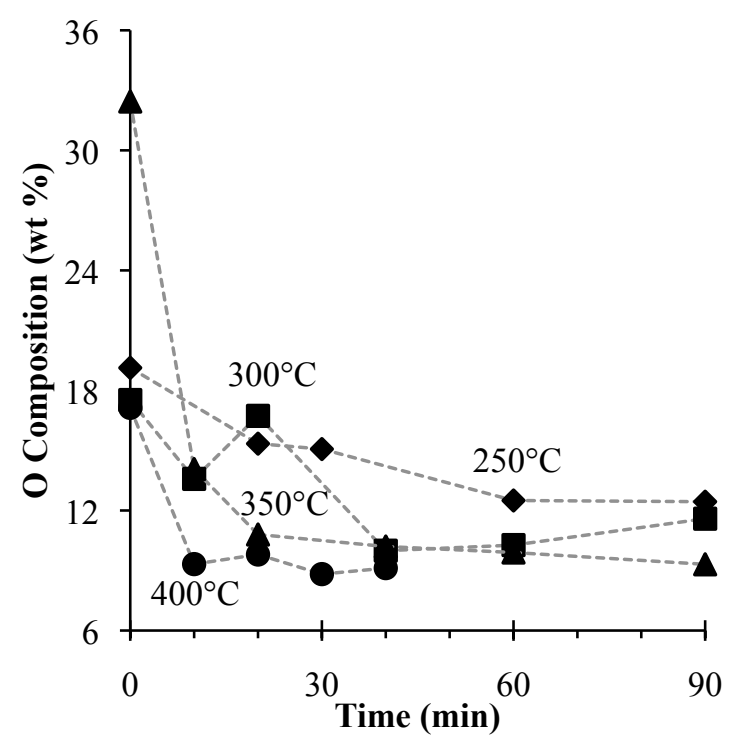

(d)

Figure 2.8 Elemental composition a) $\mathrm{C}, \mathrm{b}) \mathrm{N}, \mathrm{c}) \mathrm{S}$, and d) $\mathrm{O}$ in the heavy biocrude at $250^{\circ} \mathrm{C}(\diamond), 300^{\circ} \mathrm{C}(\boldsymbol{\square}), 350^{\circ} \mathrm{C}(\Delta)$, and $400{ }^{\circ} \mathrm{C}(\bullet)$

Figure 2.8 shows the $\mathrm{C}, \mathrm{N}, \mathrm{S}$, and $\mathrm{O}$ wt \% of the heavy biocrude. Figure 2.8 shows that the composition of $\mathrm{C}$ increases with both time and temperature. The $\mathrm{C}$ composition of the heavy crude was always lower than that of the light biocrude produced at the same conditions. Figure 2.8 shows that the $\mathrm{N}$ composition in the heavy biocrude settles to a time independent value of about $7 \pm 1$ wt $\%$, which exceeds that of the light biocrude. The sulfur 
wt \%, shown in Figure 2.8c is not a strong function of time at any of the temperatures investigated, and it has its lowest values at the intermediate liquefaction temperatures. Figure 2.8d illustrates how the composition of 0 decreased as time progressed. Increasing temperature also resulted in a lower 0 content after $20 \mathrm{~min}$. The terminal value of the 0 content of the heavy biocrude formed at a given temperature was higher than the corresponding value in the light biocrude. Jena et al. also determined the elemental content of heavy biocrude from a different alga, showing similar values for $\mathrm{C}, \mathrm{N}$, and $\mathrm{O}$ composition but nearly three times the $\mathrm{S}$ composition [10]. The higher $\mathrm{S}$ composition may be inherent to the algal species.

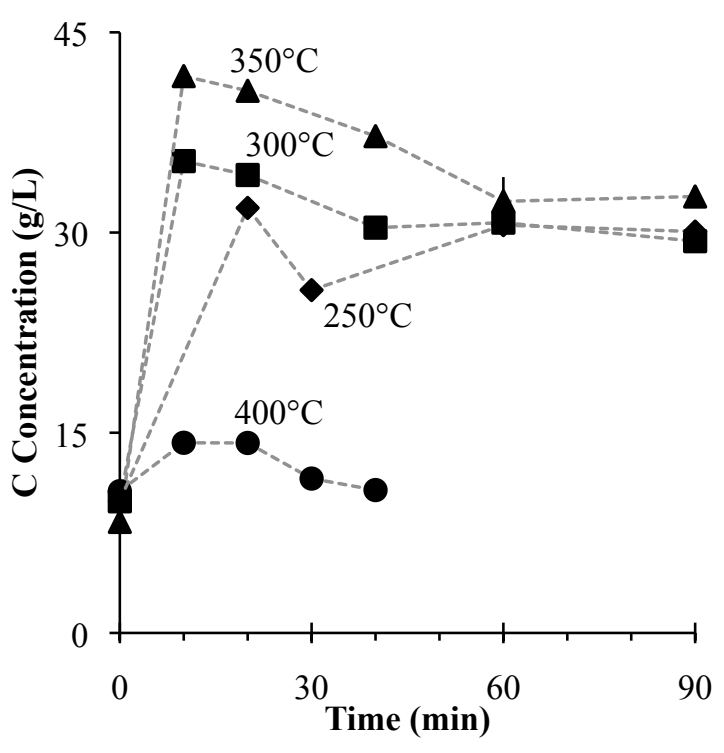

(a)

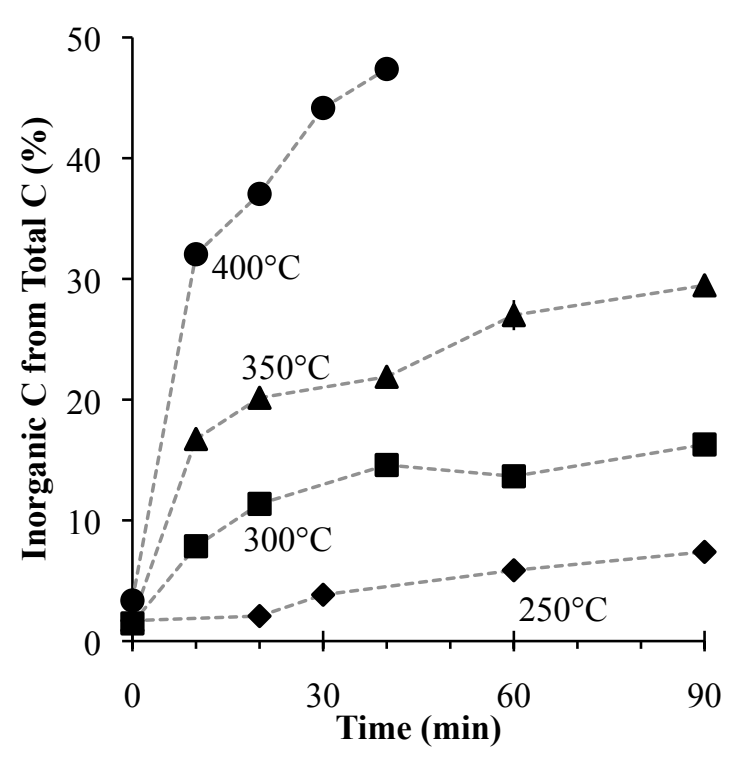

(b)

Figure 2.9 Concentration of $\mathrm{C}$ (a) and \% of $\mathrm{C}$ present as inorganic carbon (b) in the aqueous phase at $250{ }^{\circ} \mathrm{C}(\bullet), 300{ }^{\circ} \mathrm{C}$ $(\square), 350{ }^{\circ} \mathrm{C}(\Delta)$, and $400{ }^{\circ} \mathrm{C}(\bullet)$

Figure 2.9a, which shows the concentration of $\mathrm{C}$ in the aqueous phase produced after liquefaction, illustrates that the $\mathrm{C}$ concentration at a given temperature is highest at the shortest reaction time and then gradually decreases as time increases. Figure 2.9a also shows that more $\mathrm{C}$ is released into the aqueous phase at a fixed time as temperature increases, up to $350^{\circ} \mathrm{C}$. At supercritical conditions, the $\mathrm{C}$ concentration is roughly half that of subcritical conditions, perhaps because at the higher temperature, C containing 
compounds in the aqueous phase are decomposed more easily into volatile compounds. Figure $2.9 \mathrm{~b}$ shows that increased time and temperature also increase the portion of total carbon in the aqueous phase that is inorganic carbon, up to $47 \%$ at the most severe conditions.

Preliminary analysis of the aqueous phase via high-performance liquid chromatography revealed the presence of citric acid, glucose (trace), glycerol, lactic acid, acetic acid, and pyroglutamic acid. These compounds only account for $\sim 30 \%$ of the total organic $\mathrm{C}$ in the aqueous phase, so a continued investigation is still needed.

The $\mathrm{N}$ concentration in the aqueous phase is $11 \pm 1 \mathrm{~g} / \mathrm{L}$ after a short amount of hydrothermal treatment, regardless of temperature (Table 2.2). Since most of the intracellular nitrogen $(>90 \%)$ in marine microalgae resides in proteins [61], it appears that many of those proteins are readily decomposed into water-soluble amino acids and ammonia.

Table 2.3 shows the $\mathrm{C}$ composition of the solids is $52 \pm 1 \mathrm{wt} \%$ at $0 \mathrm{~min}$, which is similar to the $\mathrm{C}$ composition of the dried biomass feedstock. Upon experiencing liquefaction conditions, the $\mathrm{C}$ composition drops to as low as $5.18 \mathrm{wt} \%$ at $300{ }^{\circ} \mathrm{C}$, indicating that most of the organic material has been transferred from the solid algal biomass to the other product fractions. At $250^{\circ} \mathrm{C}$, the $\mathrm{C}$ content is relatively high, only dropping as low to as 31.7 wt \%. Table 2.3 also shows that the $\mathrm{N}$ composition in the solids is approximately $10 \mathrm{wt} \%$ initially and then drops to $<5 \mathrm{wt} \%$ by the end of the reaction. We did not include the data for sulfur in Table 2.3, as $\mathrm{S}$ in the solids is undetectable after $20 \mathrm{~min}$. Sulfur was $0.6 \mathrm{wt} \% \mathrm{in}$ the initial biomass. 
Table 2.2 Table 2. $\mathrm{N}$ Concentration in the aqueous phase $(\mathrm{g} / \mathrm{L})$

\begin{tabular}{ccccc}
\hline $\begin{array}{c}\text { Time } \\
(\min )\end{array}$ & $250{ }^{\circ} \mathrm{C}$ & $300{ }^{\circ} \mathrm{C}$ & $350{ }^{\circ} \mathrm{C}$ & $400{ }^{\circ} \mathrm{C}$ \\
\hline 0 & 2 & 2 & 1 & 2 \\
10 & $-{ }^{\mathrm{a}}$ & 11 & 11 & 12 \\
20 & 10 & 11 & 13 & 13 \\
30 & 11 & - & - & 11 \\
40 & - & 11 & 12 & 11 \\
60 & 11 & 12 & $12 \pm 2$ & - \\
90 & 11 & 12 & 14 & - \\
\hline \multicolumn{5}{c}{ aNo data available }
\end{tabular}

Table 2.3 Carbon and Nitrogen composition (wt \%) of the solids

\begin{tabular}{|c|c|c|c|c|c|c|c|c|}
\hline \multirow{2}{*}{$\begin{array}{l}\text { Time } \\
(\min )\end{array}$} & \multicolumn{2}{|c|}{$250^{\circ} \mathrm{C}$} & \multicolumn{2}{|c|}{$300^{\circ} \mathrm{C}$} & \multicolumn{2}{|c|}{$350^{\circ} \mathrm{C}$} & \multicolumn{2}{|c|}{$400{ }^{\circ} \mathrm{C}$} \\
\hline & $\mathrm{C}$ & $\mathrm{N}$ & $\mathrm{C}$ & $\mathrm{N}$ & $\mathrm{C}$ & $\mathrm{N}$ & $\mathrm{C}$ & $\mathrm{N}$ \\
\hline 0 & 53.2 & 10.5 & 51.0 & 9.21 & 51.2 & 9.98 & 52.7 & 10.1 \\
\hline 10 & $-^{a}$ & - & 21.4 & 3.77 & 22.6 & 3.22 & 12.6 & 1.29 \\
\hline 20 & 45.6 & 10.8 & 11.4 & 2.12 & 16.9 & 2.26 & 8.88 & 1.44 \\
\hline 30 & 42.5 & 8.71 & - & - & - & - & 18.3 & 2.34 \\
\hline 40 & - & - & 10.1 & 1.29 & 23.9 & 2.55 & 16.6 & 2.19 \\
\hline 60 & 43.8 & 6.41 & 5.18 & 0.88 & - & - & - & - \\
\hline 90 & 31.7 & 4.59 & 7.18 & 1.31 & 35.8 & 2.41 & - & - \\
\hline
\end{tabular}

aNo data available 


\subsubsection{Elemental Distribution}

Having reported gravimetric yields and elemental composition (wt \%) for each of the product fractions, we now discuss how the reaction conditions affected the fraction of the initial $\mathrm{C}, \mathrm{N}$, and $\mathrm{P}$ that is distributed to each product fraction.

Summing the mass of $\mathrm{C}$ in the biocrude, gases, solids, and aqueous phase and comparing that value with the mass of $\mathrm{C}$ in the initial algal biomass permits calculation of the $\mathrm{C}$ balance in each experiment. The average $C$ balance is $97 \pm 14 \%$ from all of the reaction conditions. The large standard deviation comes from the $\mathrm{C}$ balance being lower from the reactions at supercritical conditions. As previously mentioned, there is a significant amount of volatile compounds produced at supercritical conditions that is likely lost during transfers or cannot be analyzed with the gas phase products.

Table 2.4 Carbon (\%) distributed to the light and heavy biocrudes

\begin{tabular}{|c|c|c|c|c|c|c|c|c|}
\hline \multirow{2}{*}{$\begin{array}{l}\text { Time } \\
\text { (min) }\end{array}$} & \multicolumn{4}{|c|}{ Light Biocrude } & \multicolumn{4}{|c|}{ Heavy Biocrude } \\
\hline & $250^{\circ} \mathrm{C}$ & $300^{\circ} \mathrm{C}$ & $350^{\circ} \mathrm{C}$ & $400{ }^{\circ} \mathrm{C}$ & $250^{\circ} \mathrm{C}$ & $300{ }^{\circ} \mathrm{C}$ & $350^{\circ} \mathrm{C}$ & $400^{\circ} \mathrm{C}$ \\
\hline 0 & 9.0 & 9.7 & 13 & 7.0 & 1.2 & 3.4 & 4.5 & 3.7 \\
\hline 10 & $-{ }^{a}$ & 30 & 26 & 34 & - & 39 & 34 & 22 \\
\hline 20 & 24 & 31 & 28 & 38 & 23 & 38 & 29 & 18 \\
\hline 30 & 24 & - & - & 33 & 24 & - & - & 17 \\
\hline 40 & - & 32 & 34 & 33 & - & 37 & 28 & 16 \\
\hline 60 & 27 & 29 & 35 & - & 32 & 29 & 24 & - \\
\hline 90 & 19 & 29 & 37 & - & 28 & 28 & 26 & - \\
\hline
\end{tabular}

aNo data available

Table 2.4 shows that the fraction of the $\mathrm{C}$ in the algae that is partitioned to the light biocrude tends to increase with the reaction severity. That is, at a fixed reaction time, the amount of the initial $\mathrm{C}$ that appears in the light biocrude tends to increase with temperature. Likewise, at a fixed temperature, the amount tends to increase with time. After reaching its highest value of $38 \%$ at $400{ }^{\circ} \mathrm{C}$ and $20 \mathrm{~min}$, the percent of initial $\mathrm{C}$ in the 
light biocrude appears to decrease, which suggests decomposition of some of the compounds, perhaps to gases.

The data in Table 2.4 for the heavy biocrude shows a similar maximum value $39 \%$ in this case) but at much milder conditions $\left(300{ }^{\circ} \mathrm{C}, 10 \mathrm{~min}\right)$. As the reaction severity increases beyond this point, the fraction of initial $\mathrm{C}$ partitioned to the heavy biocrude decreases. This region is also where the fraction of initial $\mathrm{C}$ partitioned to the light biocrude increases, which suggests that some of the larger molecules in the heavy biocrude are being converted to smaller ones that appear in the light sub-fraction.

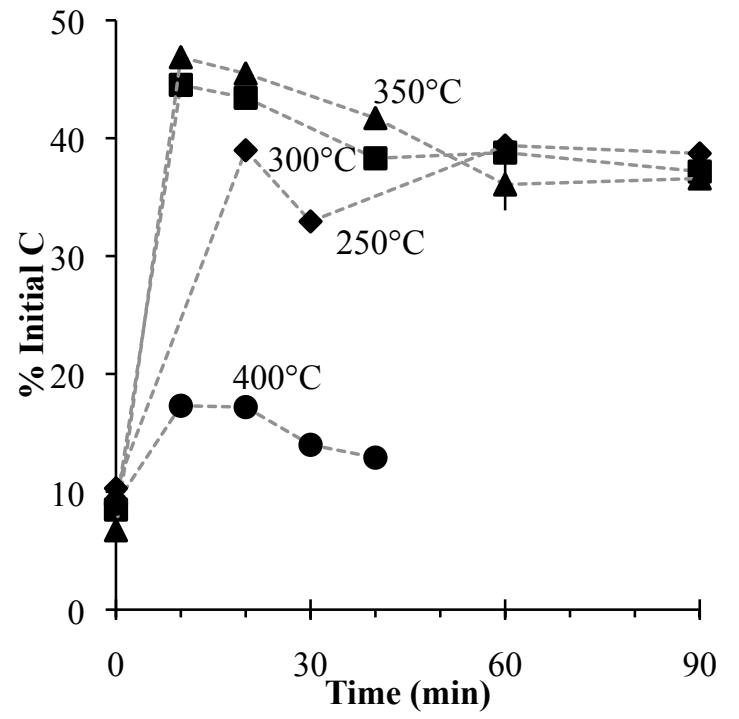

(a)

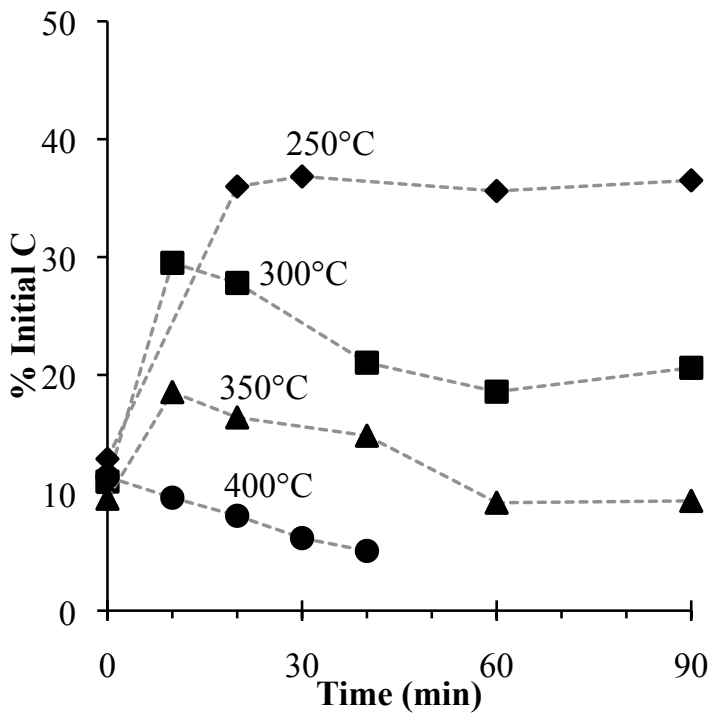

(b)

Figure 2.10 Carbon distribution in a) in the aqueous phase and b) the water-soluble products at $250{ }^{\circ} \mathrm{C}(\diamond), 300$ ${ }^{\circ} \mathrm{C}(\boldsymbol{\square}), 350{ }^{\circ} \mathrm{C}(\Delta)$, and $400{ }^{\circ} \mathrm{C}(\bullet)$

Figure 2.10a shows that much of the initial $\mathrm{C}$ appears in the aqueous phase. At subcritical conditions, approximately $40-45 \%$ of the $\mathrm{C}$ resides in the aqueous phase. Much less $C$ is in the aqueous phase from liquefaction at supercritical conditions. Figure $2.10 \mathrm{~b}$ shows that the percent of the initial $\mathrm{C}$ that resides in the dried aqueous phase (water soluble products) varies much more significantly with time and temperature than did the results in Figure 2.10a. It seems that at increasing temperatures and longer times, more and more of the C-containing compounds in the aqueous phase are lighter products that escape during evaporation of the water. 
The results presented thus far show that $>95 \%$ of the initial $\mathrm{C}$ is distributed to the biocrude and the aqueous phase. The small balance of the remaining $C$ is distributed to the gas and solid products. Less than $4 \%$ of the $\mathrm{C}$ from the biomass is converted into gas phase products at subcritical conditions, mainly as $\mathrm{CO}_{2}$. At temperatures above $250{ }^{\circ} \mathrm{C}$, the recovery of $\mathrm{C}$ in the solids is $<1 \%$.

Table 2.5 Nitrogen (\%) distributed to the light and heavy biocrudes

\begin{tabular}{cccccccccc}
\hline Time & \multicolumn{5}{c}{ Light Biocrude } & & \multicolumn{5}{c}{ Heavy Biocrude } \\
\cline { 2 - 3 } \cline { 8 - 10 }$(\min )$ & $250{ }^{\circ} \mathrm{C}$ & $300{ }^{\circ} \mathrm{C}$ & $350{ }^{\circ} \mathrm{C}$ & $400{ }^{\circ} \mathrm{C}$ & & $250{ }^{\circ} \mathrm{C}$ & $300{ }^{\circ} \mathrm{C}$ & $350{ }^{\circ} \mathrm{C}$ & $400{ }^{\circ} \mathrm{C}$ \\
\hline 0 & 1.1 & 0.8 & 1.3 & 0.7 & & 0.5 & 0.9 & 4.8 & 1.2 \\
10 & $-{ }^{\mathrm{a}}$ & 9.1 & 8.5 & 13 & & - & 26 & 21 & 13 \\
20 & 4.5 & 11 & 11 & 16 & & 16 & 23 & 17 & 10 \\
30 & 5.7 & - & - & 11 & & 17 & - & - & 10 \\
40 & - & 11 & 12 & 11 & & - & 21 & 15 & 9.0 \\
60 & 7.4 & 9.3 & 12 & - & & 21 & 14 & 13 & - \\
90 & 6.3 & 12 & 12 & - & & 19 & 16 & 13 & - \\
\hline
\end{tabular}

aNo data available

Similar to the calculation of the $\mathrm{C}$ balance, summing the mass of $\mathrm{N}$ in the biocrude, solids, and aqueous phase and comparing that value with the mass of $\mathrm{N}$ in the initial algal biomass permits calculation of the mean $\mathrm{N}$ balance for these experiments as $104 \pm 9 \%$.

Table 2.5 shows that the amount of the original $\mathrm{N}$ in the algae that is partitioned to the light biocrude levels out at about $11 \%$ after reaching moderate liquefaction conditions. Conversely, the $\mathrm{N}$ recovery in the heavy biocrude initially increases, reaches a maximum of $26 \%$, and then decreases with time at each of the three highest temperatures investigated. Although there is a significant reduction in the amount of $\mathrm{N}$ partitioned in the heavy biocrude as time progresses at the higher temperatures, the absence of a corresponding increase in the light biocrude indicates that the N-containing compounds are not simply transferred to the light biocrude. 
Table 2.6 Nitrogen (\%) distributed to the aqueous phase

\begin{tabular}{ccccc}
\hline $\begin{array}{c}\text { Time } \\
(\min )\end{array}$ & $250{ }^{\circ} \mathrm{C}$ & $300{ }^{\circ} \mathrm{C}$ & $350{ }^{\circ} \mathrm{C}$ & $400{ }^{\circ} \mathrm{C}$ \\
\hline 0 & 14 & 12 & 8 & 17 \\
10 & $-^{\mathrm{a}}$ & 75 & 67 & 83 \\
20 & 66 & - & - & 81 \\
30 & 76 & 79 & 83 & 71 \\
40 & - & 78 & 74 & 67 \\
60 & 75 & 80 & $69 \pm 10$ & - \\
90 & 82 & 81 & 84 & - \\
\hline
\end{tabular}

${ }^{\mathrm{a}}$ No data available

Table 2.6 shows that about two-thirds of the initial $\mathrm{N}$ is immediately distributed into the aqueous phase even at the mildest liquefaction conditions. Although there is some variation, the $\mathrm{N}$ distribution is always $75 \pm 9 \%$, regardless of the conditions used. Ideally, all of the $\mathrm{N}$ would be distributed to the aqueous phase so that it could possibly be recovered and re-used for algae cultivation. Also, if all of the $\mathrm{N}$ were distributed to the aqueous phase, then there would be none in the biocrude, which would also be a desirable outcome. 


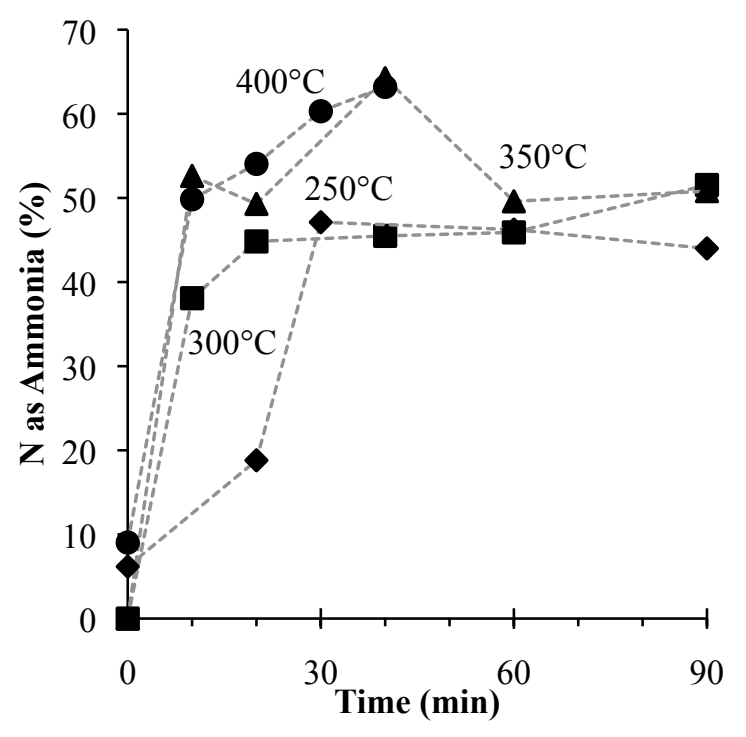

Figure 2.11 Figure 11. Percent of total nitrogen as ammonia in the aqueous phase at $250{ }^{\circ} \mathrm{C}(\bullet), 300{ }^{\circ} \mathrm{C}(\boldsymbol{\square}), 350$ ${ }^{\circ} \mathrm{C}(\Delta)$, and $400^{\circ} \mathrm{C}(\bullet)$

Figure 2.11 shows that the portion of $\mathrm{N}$ in the aqueous phase that is present as ammonia generally increases with temperature for a fixed time. For example, at 20 min, the amount of aqueous phase nitrogen present as ammonia is 19,45, 49, and 54\%, respectively, at $250,300,350$, and $400{ }^{\circ} \mathrm{C}$, respectively. This result indicates that the reactions that take place during liquefaction to convert organic nitrogen to ammonia become more favorable at higher liquefaction temperatures. The results in Figure 2.11 also show that only a portion of the aqueous-phase $\mathrm{N}$ is present as ammonia, in contrast to what others had previously assumed [7]. Most of the ammonia is likely derived from the decomposition of proteins.

At $250{ }^{\circ} \mathrm{C},<5 \%$ of the $\mathrm{N}$ is in the solids and at higher temperatures, $<1 \%$ of the $\mathrm{N}$ is distributed to the solid phase. Although $\mathrm{N}$ containing gases are not easily detected in the gas phase, Ross et al. identified $\mathrm{HCN}, \mathrm{N}_{2} \mathrm{O}$ and $\mathrm{NO}_{\mathrm{x}}$ in their experiments [19]. It is likely that they are present here only at very low concentrations since $>99 \%$ of the initial $\mathrm{N}$ in the algae appears in the biocrude and aqueous phase. 
Table 2.7 Phosphorus (\%) distributed to the aqueous phase

\begin{tabular}{ccccc}
\hline Time & \multicolumn{4}{c}{ P Distribution } \\
\cline { 2 - 5 }$(\min )$ & $250{ }^{\circ} \mathrm{C}$ & $300{ }^{\circ} \mathrm{C}$ & $350{ }^{\circ} \mathrm{C}$ & $400{ }^{\circ} \mathrm{C}$ \\
\hline 10 & $-^{\mathrm{a}}$ & 71 & 57 & 51 \\
20 & 82 & 67 & 60 & 58 \\
30 & 81 & - & - & 48 \\
40 & - & 66 & 48 & 50 \\
60 & 76 & 75 & $38 \pm 3$ & - \\
90 & 85 & 74 & 44 & - \\
\hline \multicolumn{5}{c}{ aNo data available }
\end{tabular}

Phosphorus is an important element for algal cultivation and its limited quantities emphasize the need to recover and recycle it for sustainable production of algal biofuels [62]. The $\mathrm{P}$ content of the algal feedstock is $0.6 \%$. We used this value to calculate the fraction of $\mathrm{P}$ distributed to the aqueous phase. The values of total phosphate, shown in Table 2.7, illustrate that more than half of the initial P partitioned into the aqueous phase, except at the more severe processing conditions. At a given reaction time, the amount of $\mathrm{P}$ partitioned to the aqueous phase decreases with increasing temperature. Clearly, using milder liquefaction conditions favors retention of phosphorus in the aqueous phase. Values determined for total phosphorus and phosphate were similar, demonstrating that most of the $\mathrm{P}$ in the aqueous phase is present as free phosphate. Garcia Alba et al. also reported that all of the $\mathrm{P}$ in the aqueous phase from the liquefaction of Desmodesmus sp. was in the form of phosphate [58]. The $\mathrm{P}$ content of the biocrude and solids produced at $350^{\circ} \mathrm{C}$ for $60 \mathrm{~min}$ is 0.6 and $18 \%$, respectively. These content values correspond to a $\mathrm{P}$ distribution of $26 \%$ to the biocrude and $24 \%$ to the solids. It is likely that at harsher reaction conditions more $\mathrm{P}$ may partition to the biocrude instead of to the solids or aqueous phase. This is the first time that $\mathrm{P}$ has been shown to be present in a biocrude formed via hydrothermal liquefaction of microalga. 


\subsubsection{Energy Distribution}

Table 2.8 shows that over half of the chemical energy in the algal biomass is recovered almost immediately in the biocrude, even at very mild conditions. At $300{ }^{\circ} \mathrm{C}$, about $80 \%$ of the energy in the algae is recovered in the biocrude fractions, regardless of whether liquefaction proceeded for 10,20 , or $40 \mathrm{~min}$. A similar insensitivity to reaction time is also apparent in the data from liquefaction at $350{ }^{\circ} \mathrm{C}$. This result suggests that short reaction times might be sufficient for hydrothermal liquefaction of algae, if the main objective is to convert the wet algae paste to a smaller amount of energy-dense biocrude that retains most of the chemical energy. Garcia Alba et al. reported similar values for energy recovery from a biocrude produced from Desmodesmus sp. at $300^{\circ} \mathrm{C}$ [58]. Brown et al. demonstrated that $67-90 \%$ of the energy in the alga is recovered in the biocrude and gas products [18]. The heating value of the biocrude produced in these experiments, as estimated from the Dulong equation, ranged from 35 to $38 \mathrm{MJ} / \mathrm{kg}$, values that are in accord with those reported previously for hydrothermal liquefaction of this alga [18], [20].

Table 2.8 Biocrude energy metrics at different conditions

\begin{tabular}{|c|c|c|c|c|c|c|c|c|}
\hline \multirow{2}{*}{$\begin{array}{l}\text { Time } \\
(\min )\end{array}$} & \multicolumn{4}{|c|}{ Energy distributed to the biocrude (\%) } & \multicolumn{4}{|c|}{ Energy Return on Energy Invested } \\
\hline & $250{ }^{\circ} \mathrm{C}$ & $300^{\circ} \mathrm{C}$ & $350^{\circ} \mathrm{C}$ & $400^{\circ} \mathrm{C}$ & $250{ }^{\circ} \mathrm{C}$ & $300^{\circ} \mathrm{C}$ & $350^{\circ} \mathrm{C}$ & $400^{\circ} \mathrm{C}$ \\
\hline 0 & 12 & 11 & 20 & 12 & - & - & - & - \\
\hline 10 & $-^{a}$ & $79 / 7$ & $67 / 5$ & $64 / 3$ & - & 10 & 7.0 & 5.7 \\
\hline 20 & $53 / 2$ & $78 / 7$ & $66 / 3$ & $65 / 3$ & 8.8 & 10 & 6.7 & 5.7 \\
\hline 30 & $54 / 3$ & - & - & $58 / 6$ & 8.9 & - & - & 5.1 \\
\hline 40 & - & $80 / 78$ & 71 & $56 / 4$ & - & 11 & 7.6 & 4.9 \\
\hline 60 & $68 / 6$ & $68 / 6$ & $69 / 7 \pm 3 / ?$ & - & 11 & 8.9 & $7.2 \pm 0.4$ & - \\
\hline 90 & $53 / 2$ & $66 / 4$ & $73 / 0$ & - & 8.8 & 8.6 & 7.5 & - \\
\hline
\end{tabular}

aNo data available

We calculated the energy return on energy invested (EROEI) of a hypothetical liquefaction reactor by dividing the amount of chemical energy in the biocrude produced at a given set of liquefaction conditions with the amount of energy needed to heat and pressurize the algae paste from $20^{\circ} \mathrm{C}$ and 1 atm to those liquefaction conditions. We used the steam tables to determine the enthalpies of the feed and effluent streams and assumed 
conservatively that the algae paste had the same enthalpy as water. A well-engineered, large-scale liquefaction process would incorporate heat integration such that the hot reactor effluent would be used to heat the feed stream. We assumed that $80 \%$ of the heat in the effluent can be recovered in this way. Table 2.8 shows that the EROEI exceeds unity at all conditions investigated. Even at the most harsh reaction conditions investigated, there is approximately a fivefold increase in the energy return. Keep in mind that this calculation deals only with the liquefaction reactor. The EROEI for an entire process would be lower as additional energy inputs would be required for other unit operations (e.g., mixing, separations).

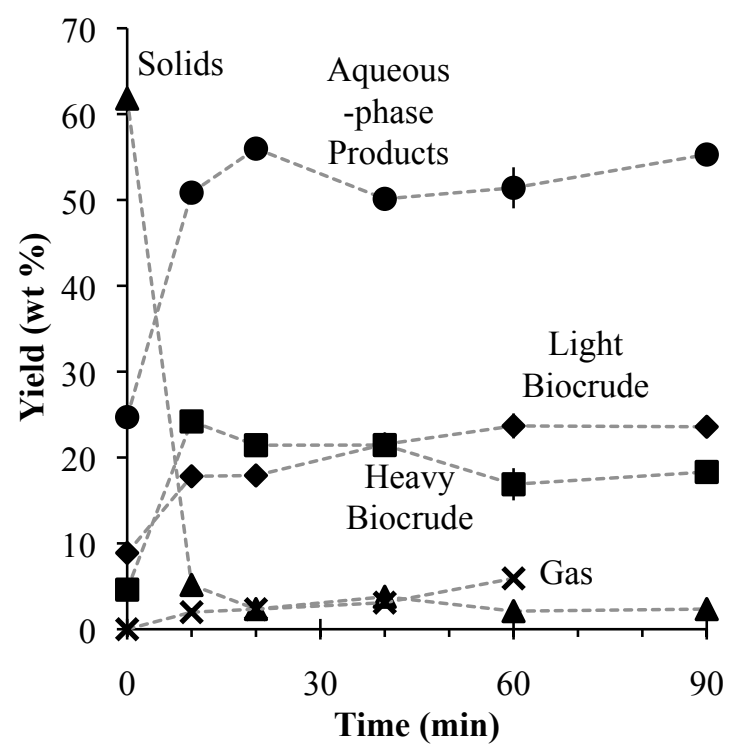

Figure 2.12 Temporal variation of yields of light biocrude $(\bullet)$, heavy biocrude $(\bullet)$ ), aqueous-phase products $(\bullet)$, solids $(\Delta)$, and gas $(X)$ at $350^{\circ} \mathrm{C}$

\subsubsection{Reaction Network}

Figure 2.12 illustrates the yields of the product fractions as they change with time at $350{ }^{\circ} \mathrm{C}$. These data, along with that at the other temperatures, can be used to deduce a potential reaction network for the liquefaction of microalgae. Most of the initial algal biomass exists as materials classified as solids in our protocol. As the reaction begins, the yields of gases, volatiles, aqueous-phase products, light biocrude, and heavy biocrude increase as the yield of solids decreases, suggesting direct reaction paths from the solids to all of the other product fractions. The reaction of solids to aqueous-phase products likely represents the release of intracellular proteins and carbohydrates and their subsequent 
decomposition in the hydrothermal environment. The pathway from solids to light and heavy biocrudes presumably represents the decomposition of the cell wall as the phospholipids are hydrolyzed. Any intracellular lipids are also released and hydrolyzed as the reaction progresses. It is likely that the aqueous-phase products and biocrudes contribute to the continued formation of gases as the reaction severity increases, thus suggesting at least a minor pathway from these product fractions to gas production. The biocrude contributes some gaseous compounds that are formed during cracking reactions [20], although it is not clear whether they originate from the light or heavy biocrude or both. The simultaneous rise in light biocrude yield as the heavy biocrude yield decreases suggests a pathway between these two product fractions. The path from heavy to light biocrude may be reversible if polymerization reactions occur as has been assumed [60]. Light and possibly heavy biocrude probably contribute to the aqueous-phase products as triglycerides and phospholipids are hydrolyzed and water-soluble glycerol and phosphates are formed. Reactions such as these could account for the slight increase in the yield of aqueous-phase products with increasing time. Using the aforementioned observations and assumptions, we offer Figure 2.13 as a potential reaction network, showing the dominant reaction directions and paths for the hydrothermal liquefaction of this microalga.

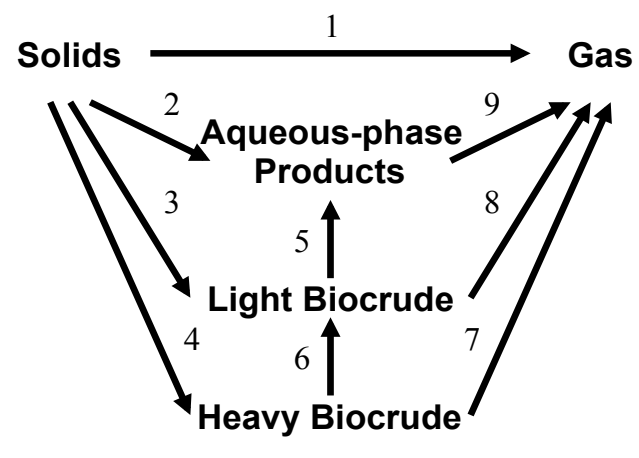

Figure 2.13 Potential reaction network for hydrothermal liquefaction of algae

\subsection{Conclusions}

Hydrothermal liquefaction at $300{ }^{\circ} \mathrm{C}$ or higher converted $\sim 95$ wt $\%$ of the initial cellular material to water- and dichloromethane-soluble compounds even at the shortest 
time examined in this study (10 min). At these short times, about half of the converted material partitioned to the aqueous phase and the other half to the biocrude product fraction. This high conversion at short times suggests that liquefaction at even shorter times should be examined. If effective, it may be possible to develop a hydrothermal liquefaction process that requires residence times of just a few minutes rather than tens of minutes. Such a process would require smaller equipment volumes and hence lower capital costs.

The lowest liquefaction temperature examined $\left(250^{\circ} \mathrm{C}\right)$ led to the partitioning of more than $80 \%$ of the initial phosphorus into the aqueous phase, primarily as phosphate. The $\mathrm{P}$ content of the aqueous phase generally decreased as the reaction severity increased (e.g., higher temperature, longer time) and it is possible that more $\mathrm{P}$ is being partitioned to the biocrude. The behavior for $\mathrm{N}$ was different, as the amount partitioned to the aqueous phase (primarily as ammonia) first increased, reached a maximum of $80-85 \%$, and then decreased as the reaction severity increased. Thus, it appears that there is a tradeoff to be considered in the liquefaction severity as different liquefaction conditions gave the highest $\mathrm{P}$ (mild conditions) and $\mathrm{N}$ (moderate conditions) recoveries in the aqueous phase. High recoveries of both are desired to facilitate nutrient recycling.

The molecular form of $\mathrm{N}$ and $\mathrm{P}$ compounds in the aqueous phase is important for nutrient recycling as well, with ammonia and phosphate being the most desirable molecules bioavailable to algae. While we confirmed that most $\mathrm{P}$ is in the form of free phosphate, only an average of $48 \%$ of the $\mathrm{N}$ present in the aqueous phase was ammonia. Bacteria have been shown to grow poorly, yet convert a large amount of organic $\mathrm{N}$ to ammonia in low organic $\mathrm{C} / \mathrm{N}$ ratio $(<10$ by weight) growth media [63]. The average organic $\mathrm{C} / \mathrm{N}$ ratio of the aqueous phase was 1.57 , so growing a microbial side-culture with it may facilitate ammonia regeneration before it is recycled to the algae operation [63].

Up to $80 \%$ of the chemical energy resident in the algal biomass can be recovered in the biocrude and in most cases the energy in the biocrude is at least 5 times greater than the unrecovered energy needed to produce it. The oxygen content in the biocrude decreased with increasing reaction severity. The nitrogen content, on the other hand, increased with 
the reaction severity until it reached a limiting value. Neither heteroatom is desired in the biocrude. The present results show that liquefaction conditions can be selected to produce bio-oil with either relatively low oxygen or nitrogen content, but not both simultaneously.

The tradeoffs noted above indicate that selecting optimal conditions for hydrothermal liquefaction will not be a simple exercise. A quantitative kinetics model that faithfully represents the experimental results reported herein would be an invaluable tool for elucidating these tradeoffs and optimizing the process. The reaction network we propose could serve as a foundation for a first-generation model.

The yield of heavy biocrude decreased with increasing reaction severity. One potential pathway for the heavy material is its overreaction to form even heavier, insoluble solid material, as often happens in pyrolytic processes. This pathway does not appear to be important under the conditions examined, however, as the yield of solids (insolubles) monotonically decreased with time and were in $<3 \mathrm{wt} \%$ yield at the most severe conditions examined. Rather, the increase in the yield of light biocrude that accompanies the decrease in yield of heavy crude as the reaction severity increases, suggests that the heavy biocrude converts to lighter, not heavier, material during this hydrothermal process.

The water density used for liquefaction at $400{ }^{\circ} \mathrm{C}$ had little effect on the yields of the different product fractions. The biomass loading (wt \%), however, did have an effect. Liquefaction with higher loadings produced higher yields of biocrude, primarily by increasing the amount of the heavy crude fraction produced.

The results reported in this article are specific to the alga used in the experiments. This alga has a very high protein content and modest lipid content. Hydrothermal liquefaction of other species, which have less protein and more lipid would probably provide biocrude in higher yield and with a lower $\mathrm{N}$ content. 


\subsection{Materials and Methods}

\subsubsection{Materials}

We purchased Nannochloropsis sp. slurry ( 35 wt \% solids) from Reed Mariculture Inc., who reported its composition to be 59 wt \% proteins, 14 wt \% lipids, and 20 wt \% carbohydrates. The slurry was diluted as needed with deionized water for experimentation. We used Optima grade (>95\%) dichloromethane and n-hexane from Fisher scientific. We purchased 99.998\% pure $\mathrm{N}_{2}$ from Metro Welding Supply Corp.

\subsubsection{Experimental Apparatus}

We assembled $4.1 \mathrm{~mL}$ mini-batch reactors using $1 / 2$ in. Swagelok $®$ port connectors made from 316 stainless steel. We capped one end of the port connector and fitted a High Pressure Equipment Co. high pressure $(30,000$ psi) valve on the other end via a $1 / 2$ in. to $1 / 8$ in. reducing union and 8.5 in. of $1 / 8$ in. 316 stainless steel tubing (0.028 in. wall thickness). The total volume added to the reactor by the valve assembly was $0.5 \mathrm{~mL}$. We loaded the reactors with enough slurry so that the liquid water would expand to fill $95 \%$ of the reactor volume at the subcritical reaction temperatures, while maintaining a constant slurry concentration of $15 \mathrm{wt} \%$. We sealed the reactors, keeping ambient air in the reactor headspace. We then placed the reactors in a Techne SBL-2 fluidized sandbath and agitated them using a Burrell Wrist-Action $®$ shaker. The shaking angle was approximately $2^{\circ}$ and the shaking speed was approximately 385 oscillations per minute, as stated by the manufacturer. A Techne TC-8D temperature controller maintained the sandbath temperature to within $\pm 2{ }^{\circ} \mathrm{C}$ of the reaction temperature. Using a similar reactor fitted with a thermocouple instead of a high-pressure valve, we measured the heat-up time to the reaction temperature to be $<3 \mathrm{~min}$. At the completion of the desired reaction time, we removed the reactors from the sandbath, quenched them in a room-temperature water bath for $5 \mathrm{~min}$ and allowed them to equilibrate to room temperature for at least $60 \mathrm{~min}$ before analyzing the products.

\subsubsection{Product Work-up, Analysis, and Metrics}

We analyzed the gas in the reactor headspace using the method outlined by Brown et al. [18]. We quantitatively measured the hydrogen, carbon dioxide, carbon monoxide, 
methane, ethane, ethene, and nitrogen in the reactor headspace after reaction. The nitrogen gas present in the reactor headspace from residual air served as an internal standard and was used to determine molar and gravimetric yields of gas products.

We opened the reactors by removing the valve assembly, and we then poured the contents into a conical tube. In most cases, the products separated naturally in the conical tube into a biocrude phase, an aqueous phase, and a solid phase. We then rinsed the reactors with $9 \mathrm{~mL}$ dichloromethane to ensure that all of the contents of the reactor were collected. We added the dichloromethane to the reactor in small aliquots $(\leq 3 \mathrm{~mL})$, agitated it, and poured the contents into the conical tube. We verified that this procedure left behind $<1 \mathrm{wt} \%$ of the original mass loaded into the reactors. After collecting the reactor contents and dichloromethane rinses, we vortexed the conical tube at $3000 \mathrm{rpm}$ for $1 \mathrm{~min}$ and then centrifuged the tube in an Eppendorf 5810 centrifuge at 500 relative centrifugal force (rcf) for $1 \mathrm{~min}$. After centrifugation, the solid products accumulated at the interface between the aqueous (top layer) and organic (bottom layer) phases. We transferred the organic phase via pipet to another tube and centrifuged the conical tube again at $1500 \mathrm{rcf}$ for $3 \mathrm{~min}$ to remove suspended solids from the aqueous phase. A control experiment verified the absence of additional solids in the organic or aqueous phase after centrifuging both phases at a higher speed for longer time. We transferred the aqueous phase to another tube via pipet. We then dried the remaining material in the conical tube in a $70{ }^{\circ} \mathrm{C}$ oven for $72 \mathrm{hr}$ to drive off residual dichloromethane and water. The dried solids were weighed and analyzed.

We removed the dichloromethane from the organic phase by flowing $\mathrm{N}_{2}$ over the organic phase tubes for approximately $6 \mathrm{hrs}$. The dichloromethane-soluble product that remained is classified as the biocrude. After removing the dichloromethane, we scraped the biocrude from the walls of the tube with a metal spatula, added $8 \mathrm{~mL}$ of $\mathrm{n}$-hexane to the tube and vortexed it at $1000 \mathrm{rpm}$ for $60 \mathrm{~min}$. We centrifuged the tube for $3 \mathrm{~min}$ at $1500 \mathrm{rcf}$ and decanted the hexane phase to another tube. We removed the hexane by flowing $\mathrm{N}_{2}$ over the hexane phase tubes for $6 \mathrm{hr}$. We classify the hexane-soluble biocrude as light biocrude, while the hexane-insoluble but dichloromethane-soluble fraction is the heavy biocrude. We weighed and analyzed the light and heavy biocrude products. We examined 
some light biocrude samples using gas chromatography with previously described methods [20].

We transferred a $500 \mu \mathrm{L}$ aliquot of the aqueous phase to a pre-weighed 1 dram vial and removed the water by flowing $\mathrm{N}_{2}$ over the vial for $6 \mathrm{hr}$. The dried material is classified as the water-soluble products and we determined its gravimetric yield and elemental composition. We analyzed the remaining aqueous phase for ammonia, total nitrogen, total carbon, inorganic carbon (carbonate and bicarbonate), total phosphorus and free phosphate (orthophosphate). To bring component concentrations into assay ranges, we diluted the pure aqueous phase 1:70 with deionized water for phosphorus and phosphate measurement, 1:200 for ammonia assay, 1:600 for carbon measurement, and 1:4000 for total nitrogen assay. We measured total carbon and inorganic carbon using a Shimadzu TOC-VCSH total organic carbon analyzer, and calculated total organic carbon (TOC) by the difference. We used Hach ${ }^{\circledR}$ Nitrogen-ammonia reagent set (high-range) test kits to measure ammonia and an established persulfate method [64] to measure total N. We measured free phosphate (orthophosphate) via an established vanadomolybdophosphoric acid method [65] and total $\mathrm{P}$ by first converting all $\mathrm{P}$ to phosphate via the oxidative digestion procedure used to measure total $\mathrm{N}$. We measured the absorbance of the analyte solutions for N and P assays with a Thermo Scientific Genesys20 or Molecular Devices Spectramax M5 spectrophotometer. To determine non-cellular aqueous nitrogen and phosphorus in the unreacted algae slurry, we centrifuged a $2.5 \mathrm{wt} \%$ slurry at $18,500 \mathrm{rcf}$, filtered the supernatant through a $0.22 \mu \mathrm{m}$ acetate filter to remove all of the microalgae cells, and measured the $\mathrm{N}$ and $\mathrm{P}$ content of the filtrate. We subtracted these background values from the aqueous phase measurements for $\mathrm{N}$ and $\mathrm{P}$ to ensure reported values are only from cellular $\mathrm{N}$ and $\mathrm{P}$, and not from algae growth media. We also measured total $\mathrm{P}$ content of unfiltered slurry. The difference between this value and that of the filtrate was used to establish initial algae solids phosphorus content.

Aqueous samples were passed through a $0.22 \mu \mathrm{m}$ acetate filter to remove particulates before analysis via High-Performance Liquid Chromatography (HPLC). Filtered samples were injected $(5 \mu \mathrm{L})$ into a stream of $0.0025 \mathrm{M} \mathrm{H}_{2} \mathrm{SO}_{4}$ flowing at $0.5 \mathrm{~mL} / \mathrm{min}$ through a 
Phenominex brand Rezex ROA-Organic Acid $\mathrm{H}^{+}$column heated to $60^{\circ} \mathrm{C}$. An Agilent 1200 Refractive Index detector was used to identify and quantify certain organic compounds.

We modified the work-up procedure to determine the yields of the various solubilitybased product fractions originally present in the algal biomass feedstock. We loaded the same amount of algae slurry into a glass test tube that we would have added to the reactors and then dried the tube in an oven at $70{ }^{\circ} \mathrm{C}$ for $72 \mathrm{hr}$ to remove the water. After adding 9 $\mathrm{mL}$ of dichloromethane to the tube and vortexing the tube at $1000 \mathrm{rpm}$ for $1 \mathrm{hr}$, we added the amount of water that would be present in the reaction system and vortexed the algae, solvent, and water for $1 \mathrm{hr}$ at $1000 \mathrm{rpm}$. After this step, the phases were easily separated using the aforementioned procedure. We measured triplicate samples of the slurry mixture and we report averages with one standard deviation being the reported uncertainty.

After drying the alga paste in an oven at $70^{\circ} \mathrm{C}$ for $72 \mathrm{hr}$, we measured the dry mass of the alga remaining to determine the wt $\%$ of solids in the algal slurry. To measure ash content, we placed the dried alga in a laboratory furnace and heated it to $250{ }^{\circ} \mathrm{C}(15$ $\left.{ }^{\circ} \mathrm{C} / \mathrm{min}\right)$. We held the sample at $250^{\circ} \mathrm{C}$ for $30 \mathrm{~min}$ and then heated the sample to $580^{\circ} \mathrm{C}(20$ ${ }^{\circ} \mathrm{C} / \mathrm{min}$ ) and held the temperature for $3 \mathrm{hr}$. We cooled the sample in a desiccator for $1 \mathrm{hr}$ and then weighed the remaining material. We identified trace metals in the microalgae by dissolving $15 \mathrm{mg}$ of algae into $3 \mathrm{~mL}$ concentrated nitric acid, diluting to $12 \mathrm{~mL}$ with deionized water, and then injecting a sample into a Varian 710ES inductively coupled plasma optical emission spectrometer. We scanned the emission results for positive identification of trace metals.

We weighed the light and heavy biocrudes, solids, and water-soluble product fractions to calculate gravimetric yields and we sent the dried alga and product fractions to Atlantic Microlab Inc. for measurement of $\mathrm{C}, \mathrm{H}, \mathrm{N}$, and $\mathrm{S}$ composition. We estimated $\mathrm{O}$ composition in the light and heavy biocrude by difference, assuming a minimal contribution of other elements were present. Midwest Microlab measured total $\mathrm{P}$ in the solid and biocrude product fractions. We calculated the heating value of the products using Dulong's formula and the $\mathrm{C}, \mathrm{H}, \mathrm{O}$, and $\mathrm{S}$ weight percentages.

$$
\mathrm{HHV}(\mathrm{MJ} / \mathrm{kg})=0.338 \cdot \mathrm{C}+1.428 \cdot(\mathrm{H}-\mathrm{O} / 8)+0.095 \cdot \mathrm{S}
$$


The gravimetric yield, elemental composition, elemental distribution, and energy distribution are defined as follows:

$$
\text { Yield }(w t \%)=(\text { Mass of Product Fraction } / \text { Mass of Alga }) \times 100 \%
$$

Elemental Composition (wt \%) $=($ Mass of Element in Product Fraction $/$ Mass of Product Fraction) $\times 100 \%$

Elemental Distribution (\%) $=$ (Mass of Element in Product Fraction $/$ Mass of Element in

$$
\text { Alga) } \times 100 \%
$$

Energy Distribution (\%) $=($ Energy in Product $/$ Energy in Alga $) \times 100 \%$

\subsubsection{Reaction Parameters}

We reacted a $15 \mathrm{wt} \%$ slurry of Nannochloropsis sp. at four different temperatures; 250, 300,350 , and $400{ }^{\circ} \mathrm{C}$. We studied times from 10 to $90 \mathrm{~min}$. At $400{ }^{\circ} \mathrm{C}$ we examined water densities of $0.3,0.4$, and $0.5 \mathrm{~g} / \mathrm{mL}$. We obtained the different water densities at supercritical conditions $\left(400^{\circ} \mathrm{C}\right)$ by loading the appropriate amount of water into the reactor. To study the effect of biomass loading we examined slurries with $5,10,15,20$, and $35 \mathrm{wt} \%$ algae. 


\section{Chapter 3}

\section{Microbial Utilization of Aqueous Co-products from Hydrothermal Liquefaction of Microalgae Nannochloropsis oculata}

\subsection{Summary}

Hydrothermal liquefaction of algae biomass is a promising technology for the production of sustainable biofuels, but the non-oil, aqueous co-product of the process has only been examined to a limited extent. The aqueous phase from liquefaction of the alga Nannochloropsis oculata (AqAl) was used to make growth media for model heterotrophic microorganisms Escherichia coli, Pseudomonas putida, and Saccharomyces cerevisiae. Growth rates, yields, and carbon/nitrogen/phosphorus uptake were measured. E. coli and P. putida could grow using $\mathrm{AqAl}$ as the sole $\mathrm{C}, \mathrm{N}$, and $\mathrm{P}$ source in media containing $10 \mathrm{vol} \%$ to $40 \mathrm{vol} \% \mathrm{AqAl}$ with the best growth occurring at $20 \mathrm{vol} \%$. S. cerevisiae could grow under these conditions only if the media were supplemented with glucose. The results indicate that in a biorefinery utilizing algae liquefaction, the aqueous co-product may be recycled via microbial cultures with significantly less dilution than previously published methods.

The entirety of the material presented in this section has been published in Bioresource Technology as follows: "Microbial utilization of aqueous co-products from hydrothermal liquefaction of microalgae Nannochloropsis oculata." Authors: Nelson, M.C., Zhu, L., Thiel, A., Wu, Y., Guan, M., Minty, J., Wang, H.Y., Lin, X.N. Bioresource Technology. 2013. 136, 522-8.

\subsection{Introduction}

Hydrothermal liquefaction is a promising technology for producing bio-fuels and substantial research is underway investigating its potential use on several feedstocks, including algae [66]. The process involves heating an aqueous suspension of biomass to $200-350{ }^{\circ} \mathrm{C}$ at pressures high enough to keep water in liquid phase. At these temperatures, macromolecules in the biomass break down into smaller molecules that may then repolymerize into a viscous "bio-crude" oil product similar to crude petroleum [59].

This type of processing avoids the costly drying steps of other algal biofuel 
production methods. It also produces bio-crude oil from lipid, protein, and carbohydrate biomass components [7], [18]. However, previous studies focused primarily on the characteristics and potential uses of the bio-crude oil product of the hydrothermal treatment, while the aqueous co-product remains largely uninvestigated. While not directly upgradable to usable fuels, this aqueous co-product contains up to $45 \%$ of the initial carbon of the biomass feedstock and the majority of other components such as nitrogen and phosphorous [11]. Disposal of this material as a waste stream would be an energetic and economic burden on a large-scale process from both a nutrient loss and wastewater-processing standpoint. A recent life cycle analysis study concluded, for example, that compared to fuels derived from terrestrial crops, algae fuels may actually cost more energy to produce, a result heavily influenced by fertilizer production energy requirement [67]. Efficient utilization of the aqueous co-product is a key factor affecting the overall sustainability of the process since the nutrients contained within it may represent a substantial amount of the energy investment. This work examines the composition of this aqueous co-product from hydrothermally treated algae (AqAl) and investigates its utility as a substrate for microbial cell culture.

Recycling AqAl as a media component for algae feedstock cultivation is one potential method for utilizing this material and has been investigated recently. However, algae growth inhibition has been observed at very dilute levels of AqAl concentration [13], [14], [68]. Even if AqAl could be recycled back to an algae growth operation, it would cause accumulation within the system of toxic compounds and substrates not utilizable by the algae. Inserting a microbial growth operation into the process may serve to detoxify the AqAl before it is fed back to the algae. For instance, phenols are known algae growth inhibitors that have been found in AqAl [14], [68], and bacterial cultures have been shown to reduce the concentrations of these compounds in wastewater [69]. Furthermore, microbes grown on AqAl could provide a supplementary source of biomass to the hydrothermal reaction operation, increasing the carbon efficiency of the whole process. It has also been proposed that an anaerobic fermentation step could convert organic carbon in the AqAl to biogas, which could be burned to power the biorefinery facility [70]. 
However, there has been little investigation regarding the quantity, quality, or toxicity of the substrates in AqAl for such an operation. The goal of this study was to investigate the culturability of model microorganisms on media containing AqAl as the sole C, N, and P sources. E. coli and S. cerevisiae were chosen since they have been extensively studied and widely utilized in industrial processes, and each have well-established methods for genetic manipulation and process scale-up. P. putida has also been studied substantially for its potential in bioremediation and was included in this work due to its robust and versatile metabolism [71].

\subsection{Overview}

The study began with the hydrothermal reaction of algae biomass and separation of the aqueous phase (AqAl) from the solid and oil products. This AqAl was used to formulate cell culture media where it was the only $\mathrm{C}, \mathrm{N}$, and P source. These media were used to aerobically culture bacteria Escherichia coli and Pseudomonas putida in 50mL tubes. After incubation, cells were separated from the supernatant and concentrations of $\mathrm{C}, \mathrm{N}$, and $\mathrm{P}$ compounds in spent media were compared to those of the initial, fresh media. Cultures were also grown in 96-well microplates in order to discern growth kinetics (through periodic measurement of optical density). This experimental setup is summarized in Figure 3.1. The growth of the yeast Saccharomyces cerevisiae on AqAl was investigated as well, though not as thoroughly due to poor initial performance. 


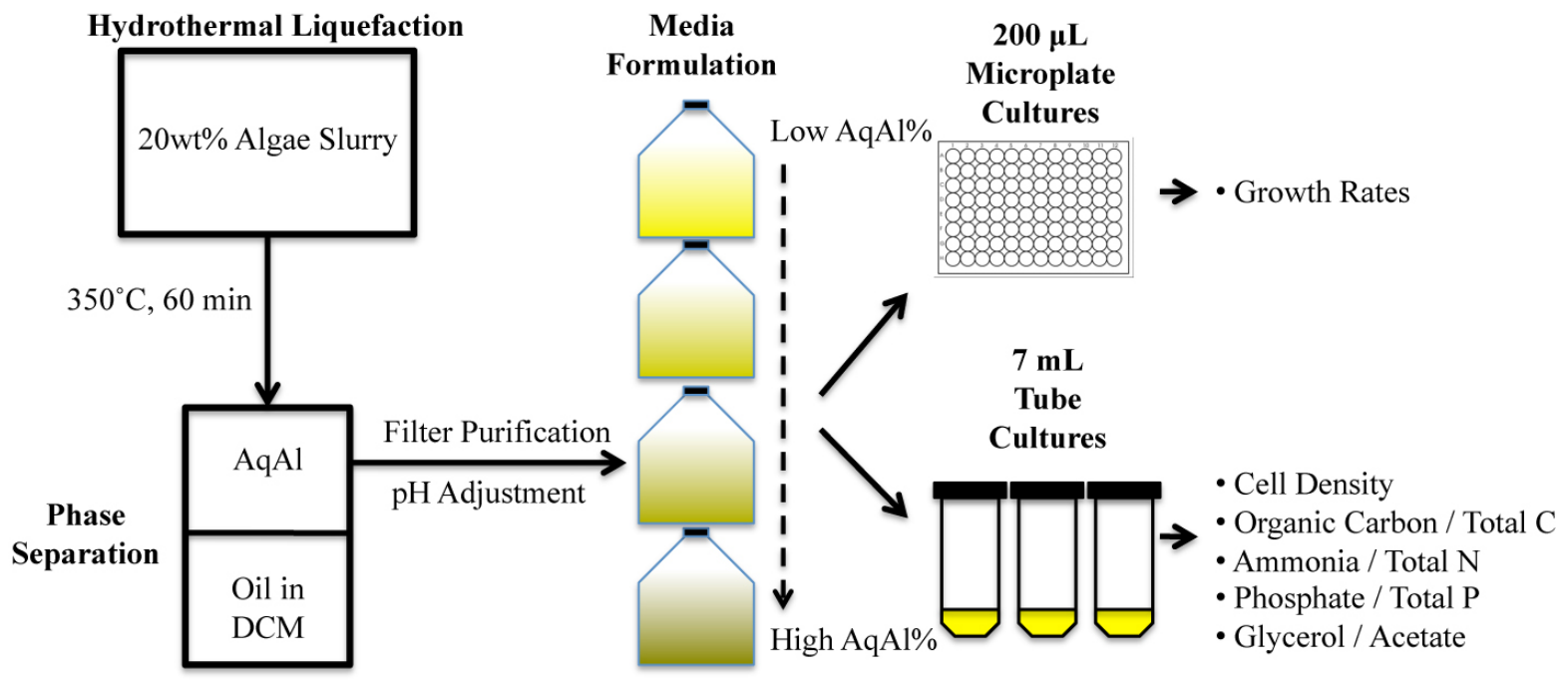

Figure 3.1 Experimental flowchart for bacterial growth studies illustrating material generation steps and the data acquired from each culture study.

\subsection{Results and Discussion}

\subsubsection{Pure AqAl Analysis}

A number of studies on algae liquefaction have collected and analyzed all products of the reaction (oil, gas, solid, and the aqueous phase) [7], [10], [11]. Among these efforts, the authors of this study recently participated in a collaborative work that utilized $N$. oculata as the feedstock algae. This work examined hydrothermal reactions of algae slurry across a range of temperatures, times, and loading conditions, followed by analysis of all products to determine energy and elemental distribution [11]. This current study specifically focuses on AqAl generated from liquefaction at $350{ }^{\circ} \mathrm{C}$ for 1 hour, a condition that produced a high yield of biocrude oil, and investigates its suitability as a microbial growth medium.

Table 3.1 shows the concentrations of carbon, nitrogen, and phosphorus measured in AqAl. Nitrogen and phosphorus have high bioavailability, with nitrogen mostly in the form of ammonia, and phosphorus entirely in the form of phosphate. It is unclear how much of the organic carbon is bioavailable, with HPLC data showing a large assortment of unknown compounds. Two common substrates for microbial growth, glycerol and acetate, were identified, but they only make up a small fraction of the total organic carbon. Even if all organic carbon was bioavailable, the atomic C:N:P ratio of $\sim 33: 19: 1$ is significantly lower in carbon than the average composition of 60:7:1 in terrestrial biomass [72] or 
106:16:1 in planktonic biomass [73]. Therefore, the growth of any heterotrophic organism in AqAl, regardless of processing conditions, should be carbon limiting. These results were expected, since previous work indicated that up to $85 \%$ of the nitrogen and phosphorus in the algae, but only $40 \%$ of the carbon, can be distributed to the AqAl at this reaction condition. Also, much of this carbon is inorganic or volatile [11]. 
Table 3.1 Concentrations of all measured chemicals in AqAl ("*” indicates all phosphorus is in the form of phosphate). Organic Carbon datum is the average of two replicate tests and +/- is one standard error of the mean. All other data are single measurements, and +/- values represent an estimate of variation using the average standard error of the mean of duplicate calibration standards.

\begin{tabular}{lr}
\hline & g/L in AqAl \\
\hline Organic Carbon & $19.70 \pm \mathbf{0 . 1 9}$ \\
\hline Glycerol & $1.01 \pm \mathbf{0 . 0 0}$ \\
\hline Acetate & $1.52 \pm \mathbf{0 . 0 1}$ \\
\hline N as Ammonia & $12.05 \pm 0.92$ \\
\hline Total Nitrogen & $13.09 \pm \mathbf{0 . 2 7}$ \\
\hline P as Phosphate* & $1.54 \pm \mathbf{0 . 0 7}$ \\
\hline
\end{tabular}

\subsubsection{Growth Performance}

Figure 3.2 shows the final optical density change of the $7 \mathrm{~mL}$ tube cultures after 48 hours of incubation. Optical density correlates directly with cell density, though the relationship is not necessarily the same between different species (i.e. a given $\mathrm{OD}_{650}$ may indicate a different dry cell weight concentration for E. coli and P. putida). These results indicate an optimal cell yield for both organisms in media containing between 20-30 vol\% AqAl and a complete inhibition of growth between 40-50 vol\%. No other studies of heterotrophic microbial growth on algae hydrothermal liquefaction aqueous product were found, so there are no published data (to the authors' knowledge) with which to compare these results. However, there have been two studies using algae feedstock and very similar hydrothermal reaction conditions in which AqAl was used to make media for algae growth. These studies showed optimal growth / complete inhibition in AqAl dilution ranges of 0$0.2 \%$ / 1-10\% [14] and 0.25-0.5\% / $0.5-2 \%$ [68]. While this study involved heterotrophic bacteria and direct comparisons cannot be made to these phototrophic algae growth experiments, it is notable that the bacteria can utilize/withstand AqAl concentrations more than one order of magnitude greater than algae can. 


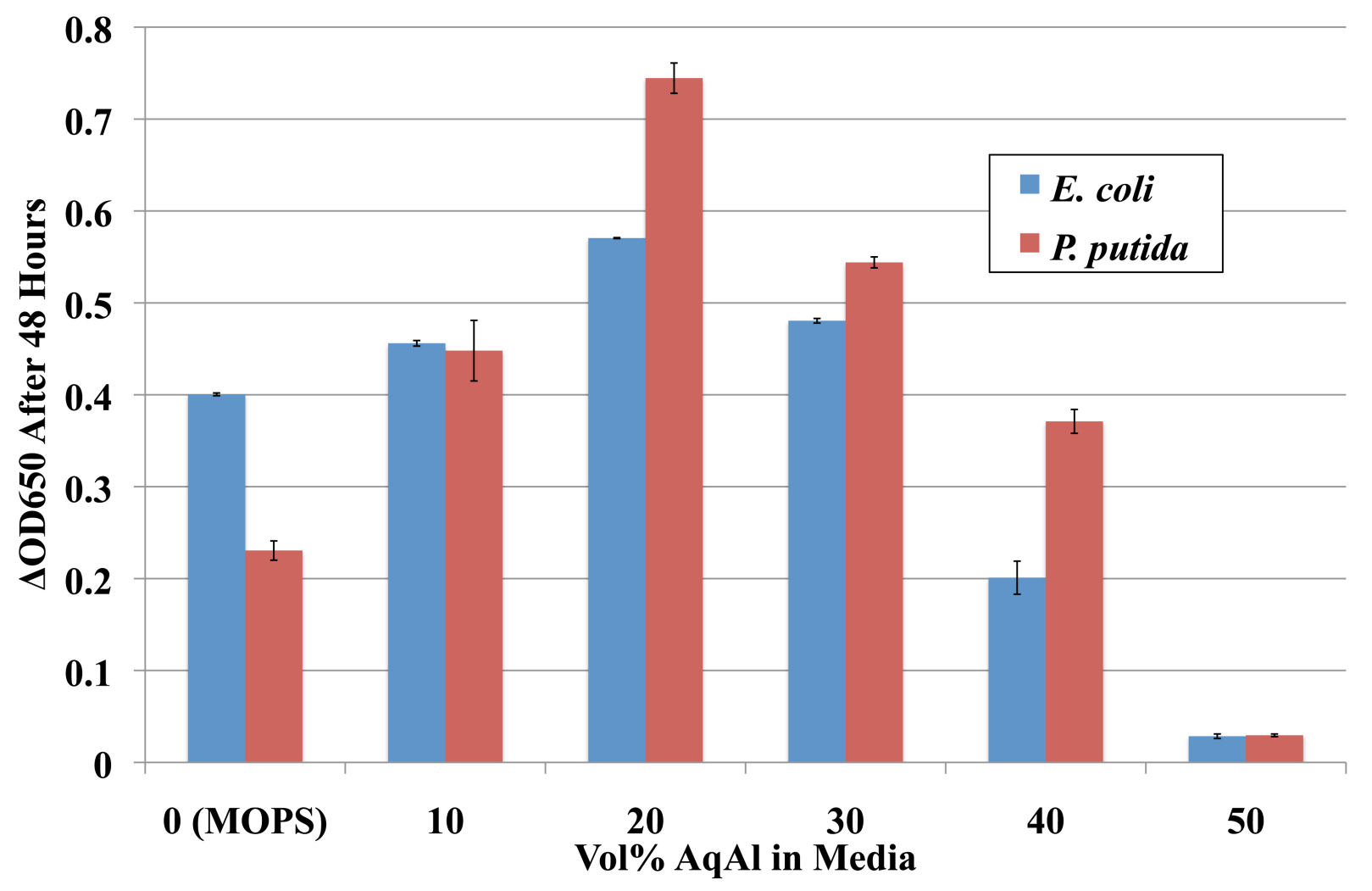

Figure 3.2 Final cell densities measured by $\Delta \mathrm{OD}_{650}$ (sample OD minus medium blank OD) of $7 \mathrm{~mL}$ culture experiments after 48-hour incubation. Control medium (MOPS) contained $2 \mathrm{~g} / \mathrm{L}$ glucose, $1 \mathrm{~g} / \mathrm{L}$ ammonium chloride, and $0.23 \mathrm{~g} / \mathrm{L}$ potassium phosphate. Other media used AqAl as the sole source of $\mathrm{C}$, $\mathrm{N}$, and $\mathrm{P}$. Data presented are averages of two replicate cultures and error bars represent one standard error of the mean.

Figure 3.3 shows growth rate results from the microplate culture test. Figure 3.3A provides an example of the $\mathrm{OD}_{650}$ vs. time data for the $20 \mathrm{vol} \%$ AqAl test. Multiple $\log$ growth phases are evident for each organism, indicating growth on multiple carbon sources. E. coli exhibits typical diauxic growth in all AqAl media, with an initial log growth phase corresponding to a preferred carbon source, followed by a brief lag phase, then a second log growth phase with a lower corresponding $\mu_{\max }$ value (Figure 3.3C). P. putida shows three log growth phases at lower AqAl concentrations and two log growth phases at higher AqAl concentrations, which suggests certain compounds are not metabolized at high AqAl concentrations ( Figure 3.3D). It could also indicate that multiple carbon sources are consumed simultaneously or that the lag time and $\mu_{\max }$ difference between two carbon sources are too small to be detected. Figure 3.3B provides a measurement of the average growth rate, calculated by dividing the $\Delta \mathrm{OD}_{650}$ achieved over all log growth phases by the total log growth time (including intermediate lag phases). E. coli shows no obvious trend in 
the different AqAl media, while P. putida shows increasing average growth rates with increasing AqAl concentrations.
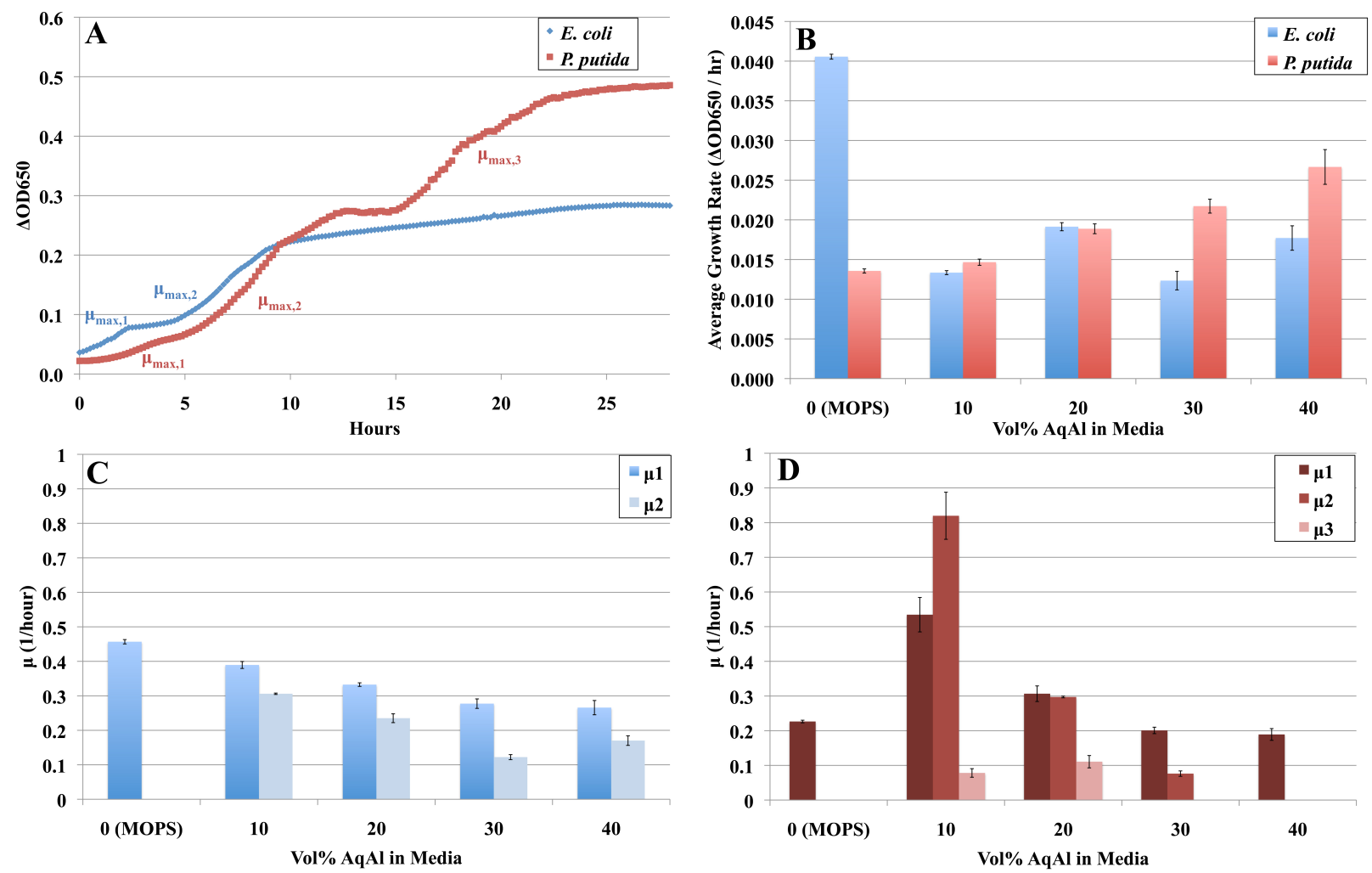

Figure 3.3 Growth rate results from microplate culture test. A) An example of growth curve data obtained for both bacteria in 20 vol\% AqAl medium, with different log growth phases labeled. B) The average growth rate $\left(\Delta 0_{650}\right.$ per hour) of both bacteria in 0-40 vol\% AqAl. C) $\mu_{\max }$ values calculated for each log growth period for $E$. coli. D) $\mu_{\max }$ values calculated for each log growth period for $P$. putida. Data presented in B, C, and D are averages of three replicate cultures and error bars represent one standard error of the mean.

\subsubsection{Substrate and Nutrient Analysis}

Elemental analyses on spent media of $7 \mathrm{~mL}$ bacterial tube cultures (after cells were separated via centrifugation) could not detect a statistically significant difference in ammonia, total nitrogen, phosphate, or total phosphorus concentration compared to initial media. This indicates that the bacterial growth was not limited by nitrogen or phosphorus. Organic carbon differences, however, were significant and are summarized in Figure 3.4A (as a comparison of absolute values) and Figure 3.4B (as percentage differences from initial media). Carbon consumption efficiency was highest in $10 \mathrm{vol} \% \mathrm{AqAl}$ at a level of $45 \%$, decreasing with increasing $\mathrm{AqAl}$ concentration to $~ 5 \%$ in 40 vol\% AqAl. Figure $3.4 \mathrm{C}$ and D 
show glycerol and acetate consumption, respectively. Both compounds were well metabolized at low AqAl concentrations, but not at 40 vol\% AqAl. Smaller amounts of each compound were consumed at this AqAl concentration, and acetate was actually produced by E. coli, potentially due to metabolic perturbations caused by AqAl [74]. 

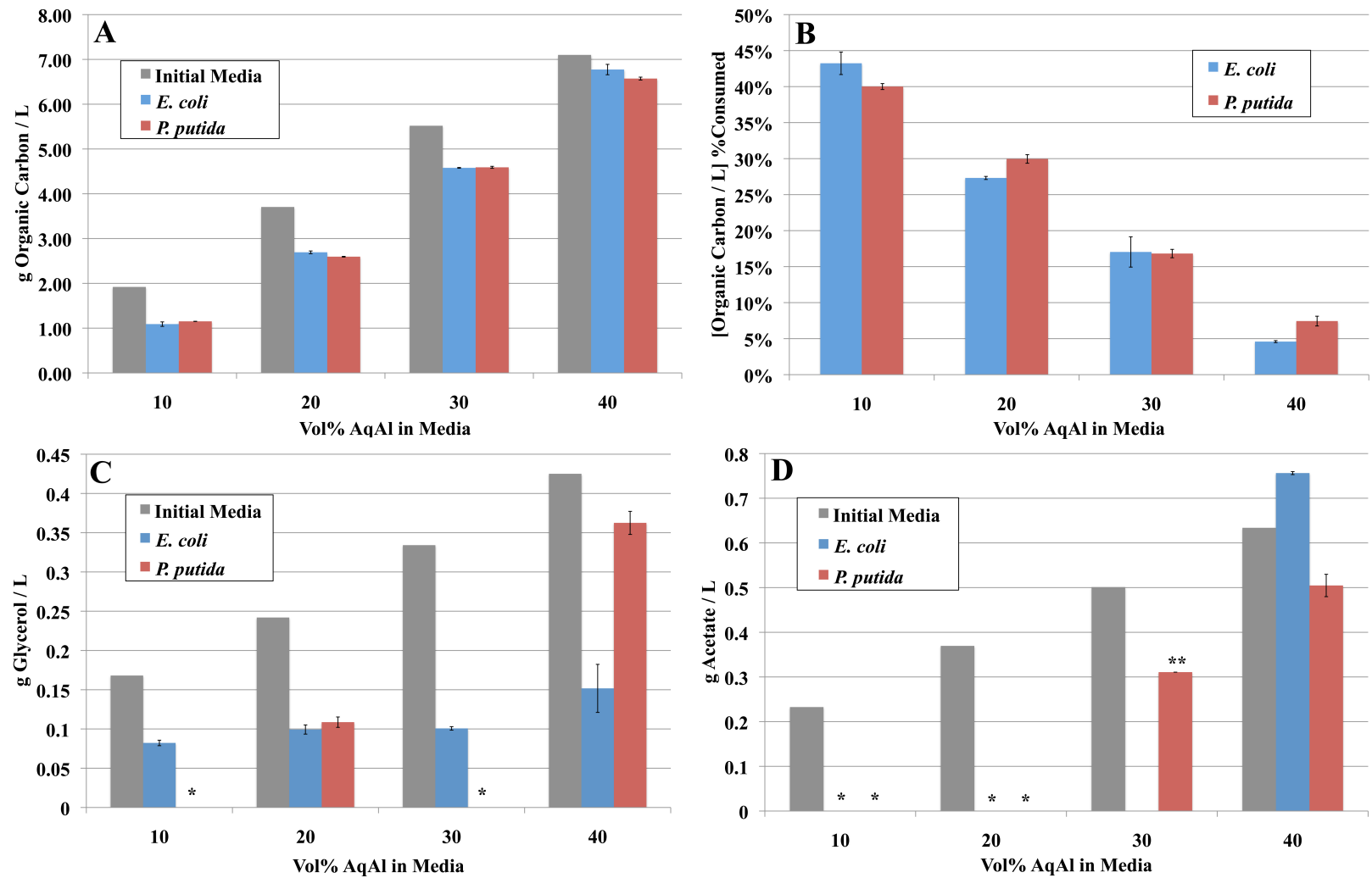

Figure 3.4 Carbon consumption data from $7 \mathrm{~mL}$, 48-hour culture experiments. A) Organic carbon in initial media compared to organic carbon remaining after incubation. B) Percentage of organic carbon consumed in each experiment. C) Glycerol in initial media compared to glycerol remaining after incubation. D) Acetate in initial media compared to acetate remaining after incubation. Initial media data presented are from single measurements, and other data presented are the average of two replicate cultures. Error bars represent one standard error of the mean. "*” indicates both replicates are below the $\sim 0.05 \mathrm{~g} / \mathrm{L}$ limit of detection. "**” indicates the value of only one replicate is shown, and the other was below the limit of detection. Note: MOPS medium (not shown) contained $2 \mathrm{~g} / \mathrm{L}$ glucose initially, which was completely consumed in all control experiments, as verified via HPLC.

\subsubsection{Yeast Growth Tests}

Figure 3.5 shows the results from a series of culturing experiments starting with AqAl media spiked with $5 \mathrm{~g} / \mathrm{L}$ of glucose and gradually decreasing glucose concentration in increments of $1 \mathrm{~g} / \mathrm{L}$ glucose every 48 hours (inoculating a new culture each time with cells from the previous one). Even when gradually acclimated to AqAl over many generations, $S$. cerevisiae showed barely measurable growth without glucose even at low AqAl concentrations. A control test was also performed in tandem with this experiment where the same initial yeast culture was grown and passaged six times into a medium containing no AqAl and a constant $5 \mathrm{~g} / \mathrm{L}$ glucose. The resulting cultures reached final $\Delta \mathrm{OD}_{650}$ values consistently between 0.8 and 0.9 , indicating the cells should have remained viable over the time period of the tests. These results suggest that AqAl has a strong inhibitory effect on $S$. 
cerevisiae, yet its growth in the presence of glucose indicates that substrate selectivity may have contributed to the yeast's poor performance compared to E. coli and P. putida.

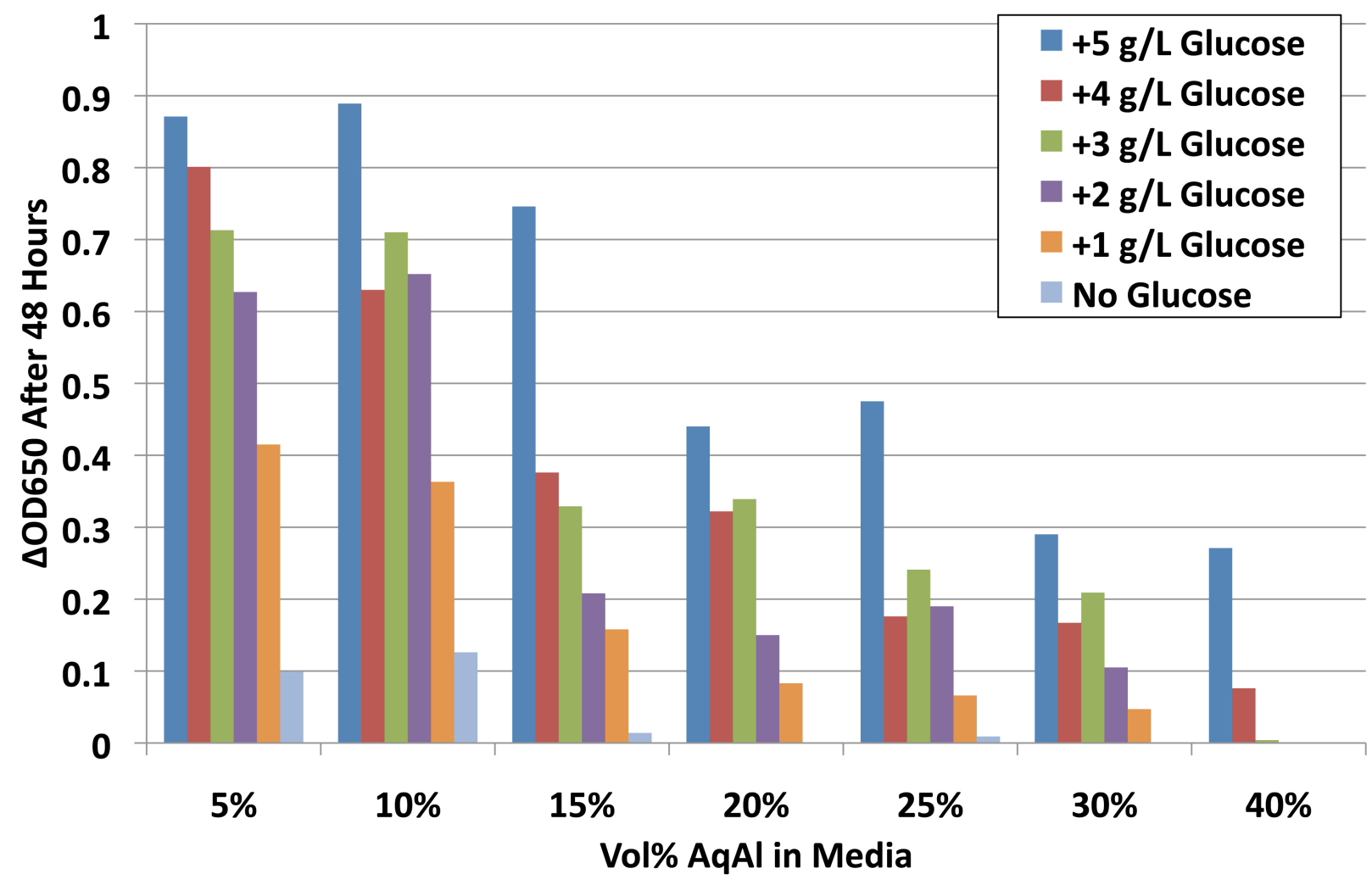

Figure 3.5 Final $\Delta \mathbf{O D}_{650}$ of $2 \mathrm{~mL}$ S. cerevisiae cultures after 48-hour incubation in glucose-spiked media. Cells from each glucose level (starting with $5 \mathrm{~g} / \mathrm{L}$ ) were used to inoculate the corresponding culture with $1 \mathrm{~g} / \mathrm{L}$ less glucose. All data are from single culture measurements.

\subsection{Conclusion}

E. coli and P. putida exhibited growth in culture media containing AqAl as the sole source of C, N, and P. Growth was the best at $20 \mathrm{vol} \% \mathrm{AqAl}$ and ceased at $50 \mathrm{vol} \%$, which indicates bacterial tolerance and utilization of AqAl in concentrations higher than those reported in published algae growth studies. These data indicate that a microbial side culture in an algae-based fuel production process may potentially provide value as a source of supplementary biomass, a detoxification step for AqAl recycle, or pretreatment for waste disposal. 


\subsection{Materials and Methods}

\subsubsection{Hydrothermal Liquefaction}

Nannochloropsis oculata (a strain often used for hydrothermal liquefaction studies due to high bio-crude oil yields and commercial availability) algae slurry was purchased from Reed Mariculture Inc. as the source of biomass. The material was $~ 35 \mathrm{wt} \%$ solids (the remainder as water), and composed of $59 \mathrm{wt} \%$ proteins, $14 \mathrm{wt} \%$ lipids, and $20 \mathrm{wt} \%$ carbohydrates on a dry basis, as reported by the supplier. Deionized water was added to the slurry to adjust it to $20 \mathrm{wt} \%$ solids content before reaction. For each reaction, $150 \mathrm{~mL}$ of 20 wt $\%$ slurry were loaded into a Parr 4570 Pressure Reactor with a calculated total volume of $360 \mathrm{~mL}$. A LC Miller PR-15AB induction coil heater was used to raise the temperature to $350^{\circ} \mathrm{C}$ and then hold it for a period of one hour. The average heat-up time for each reaction was 8.7 minutes and the temperature was maintained within $2^{\circ} \mathrm{C}$ of the target. The reactor was agitated by an impeller at 800 RPM and reached a stable pressure of 100 bar during the reaction. After one-hour of reaction time, the reactor was cooled to room temperature and the gas product was vented. The contents of the reactor (consisting of solid, aqueous, and oil products) were mixed with $100 \mathrm{~mL}$ of dichloromethane (Optima grade, $>95 \%$ purity) and transferred to a glass separatory funnel. The dichloromethanesoluble product fraction was defined as the "biocrude oil" product and the remaining water-soluble phase as the aqueous product (AqAl). This is a common practice among researchers investigating hydrothermal liquefaction of algae [7], [14]. After one hour of equilibration time, the AqAl was decanted from the oil phase. The AqAl was further purified by vacuum filtration through a Corning $0.2 \mu \mathrm{m}$ cellulose acetate filtration apparatus in order to remove residual oil and solids. This final product was considered "raw AqAl". This entire reaction and separation process was repeated three times in order to generate enough AqAl for bacterial growth experimentation and the resulting batches were combined. Hydrothermal liquefaction reactions to generate AqAl for yeast growth tests were done on a separate date, and varied slightly from the above method in that they used a $15 \mathrm{wt} \%$ algae slurry. Previous work has indicated that distribution of products for hydrothermal reactions of this alga vary little between $15 \mathrm{wt} \%$ and $20 \mathrm{wt} \%$ [11]. 


\subsubsection{Media formulation}

The medium for the E. coli and P. putida growth tests was formulated based on a standard organic-buffered 3-(N-morpholino)propanesulfonic acid (MOPS) medium for enterobacteria (Neidhardt et al., 1974). It contained $0.04 \mathrm{M}$ MOPS and $0.004 \mathrm{M}$ tricine as buffers, adjusted to a $\mathrm{pH}$ of 7.4 with $\mathrm{KOH}$. The $\mathrm{C}, \mathrm{N}$, and P sources were $2 \mathrm{~g} / \mathrm{L}$ glucose, $1 \mathrm{~g} / \mathrm{L}$ $\mathrm{NH}_{4} \mathrm{Cl}$, and $0.23 \mathrm{~g} / \mathrm{L} \mathrm{K}_{2} \mathrm{HPO}_{4}$. The medium also contained $2.91 \mathrm{nM}\left(\mathrm{NH}_{4}\right)_{6} \mathrm{Mo}_{7} \mathrm{O}_{24}\left(\mathrm{H}_{2} \mathrm{O}\right)_{4}$, 401.1nM H $\mathrm{H}_{3} \mathrm{BO}_{3}, 30.3 \mathrm{nM} \mathrm{CoCl}{ }_{2}\left(\mathrm{H}_{2} \mathrm{O}\right)_{6}, 9.61 \mathrm{nM} \mathrm{CuSO}_{4}\left(\mathrm{H}_{2} \mathrm{O}\right)_{5}, 51.4 \mathrm{nM} \mathrm{MnCl}_{2}, \quad 6.1 \mathrm{nM}$ $\mathrm{ZnSO}_{4} \cdot \mathrm{H}_{2} \mathrm{O}, 0.01 \mathrm{mM} \mathrm{FeSO}_{4}\left(\mathrm{H}_{2} \mathrm{O}\right)_{7}, 1 \mathrm{mM} \mathrm{Mg}_{2} \mathrm{SO}_{4}, 0.1 \mathrm{mM} \mathrm{CaCl}_{2}$, and $0.5 \mathrm{~g} / \mathrm{L} \mathrm{NaCl}$. All ingredients were combined with deionized water and sterilized with a Corning $0.2 \mu \mathrm{m}$ vacuum filtration system. This medium was used for control experiments and will be referred to as "MOPS medium". "Stripped" MOPS medium was also prepared which did not contain glucose, $\mathrm{NH}_{4} \mathrm{Cl}, \mathrm{K}_{2} \mathrm{HPO}_{4}$, or $\mathrm{NaCl}$. AqAl (neutralized from its initial pH of $\sim 10$ to a pH of 7 using $\mathrm{HCl}$ ) was combined with stripped MOPS medium to make mixtures of $10-50$ vol\% AqAl media.

The medium for Saccharomyces cerevisiae growth tests consisted of Difco Yeast Nitrogen Base Without Amino Acids or Ammonium Sulfate (YNB) buffer/nutrient mix, which was used at $1.7 \mathrm{~g} / \mathrm{L}$ with deionized water, along with $5 \mathrm{~g} / \mathrm{L}$ glucose and $5 \mathrm{~g} / \mathrm{L} \mathrm{NH}_{4} \mathrm{Cl}$ added as the $\mathrm{C}$ and $\mathrm{N}$ sources. This was used as the control medium and will be referred to it as "YNB medium". A stripped version of this medium was also prepared without glucose or ammonia and mixed with AqAl (adjusted to a pH of 6 with $\mathrm{HCl}$ ) to make $5-40 \% \mathrm{AqAl}$ media.

\subsubsection{Cell Culture}

For growth experiments, cryogenically preserved $\left(-80^{\circ} \mathrm{C}\right)$ stocks of E. coli $\mathrm{K} 12$ MG1655, P. putida KT2440, and S. cerevisiae S288C were recovered in 2mL of LuriaBertani (LB) rich media (for bacteria) or yeast peptone dextrose (YPD) media (for yeast) for 24 hours at $30^{\circ} \mathrm{C}$ in a New Brunswick E24 incubator agitated at $250 \mathrm{rpm}$. All cultures were contained in tightly capped $15 \mathrm{~mL}$ Falcon tubes. Milliliter aliquots of each culture were "washed" by centrifuging cells at 16,000 rcf for 1 minute in an Eppendorf 5415C microcentrifuge, decanting supernatant, and resuspending the cell pellet with an equal volume of sterile, deionized water. These cell suspensions were used at a ratio of $20 \mu \mathrm{L}$ to 2 
$\mathrm{mL}$ medium, approximately 7.5E6 cells per $\mathrm{ml}$ [75], to inoculate new individual cultures of MOPS (for bacteria), YNB (for yeast), and all AqAl-containing media. These cultures were incubated for 48 hours, after which the optical density at $650 \mathrm{~nm}\left(\mathrm{OD}_{650}\right)$ was measured with a Molecular Devices Versamax plate reader. Although optical density at $600 \mathrm{~nm}$ is more commonly used to gauge cell densities in bacterial studies, it was found that AqAl had a strong native absorbance at this wavelength and thus $\mathrm{OD}_{650}$ was used. Milliliter aliquots of each culture were washed, centrifuged, and resuspended with sterile, deionized water such that $\mathrm{OD}_{650}$ equaled 2, approximately $2.2 \mathrm{E} 9$ cells per $\mathrm{ml}$ [75]. The resulting cell suspensions were used as inocula for either tube or plate culture tests. It is common to inoculate with cells recovered from cryopreservation in rich media, but initial tests conducted in this fashion showed very long lag times ( $>72$ hours) with AqAl-containing media. The additional 48-hour intermediate step was used to acclimate the cells to the exact media used for the final growth test. Inoculum cultures of both E. coli and P. putida in $50 \% \mathrm{AqAl}$ did not exhibit measurable growth, so the $40 \%$ AqAl-acclimated cells were used to inoculate the final experiments for both $40 \%$ and $50 \%$ AqAl media.

S. cerevisiae cultures in AqAl media exhibited only slight growth $\left(\Delta \mathrm{OD}_{650}<0.15\right)$ in $5 \%$ and $10 \% \mathrm{AqAl}$ media, and no measurable growth at higher AqAl concentrations. In an effort to slowly adapt the yeast to $\mathrm{AqAl}$, a study was performed where cultures were grown for 48 hours in $2 \mathrm{~mL} \mathrm{AqAl-containing} \mathrm{yeast} \mathrm{media} \mathrm{spiked} \mathrm{with} 5 \mathrm{~g} / \mathrm{L}$ of glucose, then washed and used to inoculate fresh media containing $4 \mathrm{~g} / \mathrm{L}$ glucose. This procedure was repeated in $1 \mathrm{~g} / \mathrm{L}$ glucose increments until glucose was eliminated. No change was noticed in the performance of $S$. cerevisiae on glucose-free AqAl media, so the organism was not included in further microplate and large-volume tube tests.

Standard 96-well microplates were used for a microculture study on E. coli and $P$. putida. Three replicates of $200 \mu \mathrm{L}$ of each medium were inoculated with $4 \mu \mathrm{L}$ of the corresponding inoculum. One well of each medium was left sterile as a blank. The plate was shaken continuously and incubated at $30^{\circ} \mathrm{C}$ for 72 hours in a Molecular Devices Versamax plate reader. The $\mathrm{OD}_{650}$ of each well was measured in 10-minute intervals. 
While the microplate test was useful for measuring growth kinetics, the $200 \mu \mathrm{L}$ culture volume was not large enough for chemical analysis, so larger-volume growth tests were conducted in sealed $50 \mathrm{~mL}$ Falcon tubes. Two duplicate samples of $7 \mathrm{~mL}$ of each medium were inoculated with $25 \mu \mathrm{L}$ of the corresponding inoculum and incubated at $30^{\circ} \mathrm{C}$ for 48 hours at $250 \mathrm{rpm}$ agitation in a New Brunswick E24 incubator. The final $\mathrm{OD}_{650}$ of each culture was measured and each tube was centrifuged at 17,500 rcf for 5 minutes in an Eppendorf 5810R centrifuge in order to separate cells from spent media. The supernatant was withdrawn and stored it at $4^{\circ} \mathrm{C}$.

\subsubsection{C, N, and P Analysis}

Raw AqAl, fresh media, and spent media from the $7 \mathrm{~mL}$ growth tests were subjected to several assays to determine the concentrations of various carbon, nitrogen, and phosphorus compounds. Concentrations of the following chemicals were measured in each sample: ammonia, total nitrogen, phosphate, total phosphorus, total/inorganic/organic carbon, glycerol and acetate.

Hach Brand "Nitrogen-ammonia reagent set, high range" (product \#2606945) premade kits were used to measure nitrogen content present as ammonia. A potassium persulfate/boric acid/sodium hydroxide reagent method established for use in seawater was utilized to measure total nitrogen [64]. Free phosphate (orthophosphate) was measured via an established vanadomolybdophosphoric acid method [65]. Total phosphorus was measured by performing the phosphate assay on samples pre-processed with the total nitrogen digestion, since the reagent involved also converts all phosphorus to phosphate [64]. All ammonia, total nitrogen, phosphate, and total phosphorus tests were colorimetric and data were gathered via either a Thermo Scientific Genesys20 or Molecular Devices Spectramax M5 spectrophotometer. Total carbon and inorganic carbon were measured with a Shimadzu TOC-VCSH total organic carbon analyzer, and organic carbon was inferred by difference. Glycerol and acetate were measured via high-performance liquid chromatography (HPLC). Samples of $5 \mu \mathrm{L}$ were injected into a mobile solvent stream of $0.005 \mathrm{~N} \mathrm{H}_{2} \mathrm{SO}_{4}$ flowing at $0.5 \mathrm{~mL} / \mathrm{min}$ through a Phenominex Rezex ROA-Organic Acid $\mathrm{H}^{+}$ column. Individual, separated compounds were analyzed with an Agilent 1200 Series 
Refractive Index Detector. Glycerol and acetate were identified and quantified in samples via comparison to the elution time and peak area, respectively, of standards.

\subsubsection{Data Analysis}

All optical density data for cell cultures (both microplate and tube tests) will be presented as the difference in $\mathrm{OD}_{650}$ between cell culture and blank media $\left(\Delta \mathrm{OD}_{650}\right)$. This is preferential to absolute $\mathrm{OD}_{650}$ since the media varies in initial $\mathrm{OD}$ depending on the AqAl fraction.

Specific growth rates $\left(\mu_{\max }, \mathrm{h}^{-1}\right)$ of the microplate cultures were quantified via linear regression of $\ln \left(\mathrm{OD}_{650}\right)$ vs. time $(\mathrm{h})$ over the time interval corresponding to log growth phase. Most of the AqAl-containing cultures showed multiple log growth phases with different growth rates, so one $\mu_{\max }$ was calculated for each phase. An average growth rate was also calculated as defined by the ratio of combined $\Delta \mathrm{OD}_{650}$ of all log growth phases divided by total time of all log growth phases.

The total carbon and total nitrogen values measured in bacterial media were adjusted to account for the non-bioavailable organic carbon and nitrogen that the MOPS/tricine buffer added. All values reported represent total carbon and nitrogen due to AqAl. 


\section{Part II}

\section{Improvement of bacterial strain growth on AqAl through adaptive evolution, and investigation of tolerance and substrate utilization phenotypes through genome and transcriptome sequencing}

\section{Chapter 4}

\section{Adaptive Evolution of Bacteria Improves Growth on Aqueous Co-Products from Hydrothermal Liquefaction of Microalgae Nannochloropsis Oculata}

\subsection{Summary}

Model bacteria Escherichia coli and Pseudomonas putida were grown for $\sim 300$ cell division generations in media containing the aqueous phase (AqAl) generated from hydrothermal liquefaction of algae biomass, a product that is inhibitory to microbial growth at high concentrations. Strains of each species were isolated that had evolved improved growth phenotypes. The full genomes of these species were sequenced to identify the single nucleotide polymorphisms (SNPs) acquired. Six of these SNPs (one each from genes ilvI, ilvN, ilvH, and ptsP, and two from $g l p K$ ) were individually inserted into wild type E. coli via homologous recombination and their growth phenotypes were studied in a variety of selective media.

Additionally, the top-performing evolved E. coli strain, as well as its parent strain, were grown in both chemically defined minimal medium and AqAl medium, and RNA samples were taken for full transcriptome sequencing. The gene expression levels of each strain in the different growth conditions were compared in order to determine transcriptional alterations acquired through evolution.

\subsection{Introduction}

Hydrothermal liquefaction (HTL) is a promising technology for converting algae biomass into "biocrude" oil, a potential drop-in substitute for petroleum. It avoids the land usage and cellulose hydrolysis issues associated with biofuel formation from terrestrial 
crops, and also has a more complete biomass conversion than algae transesterification processes that only convert lipids into biofuels [66]. However, the aqueous algae coproduct (AqAl) of HTL can contain up to $40 \%$ of the carbon initially present in the algae biomass, and the majority of the nitrogen and phosphorus [7], [11]. Efficient recycle of this product within the process is critical for overall financial and material sustainability and ideally, it could be fed back to an algae growth operation to recycle nutrients. Yet research into this has found that some of the compounds, for example, organically bound nitrogen, do not appear to be bioavalable to algae [17]. AqAl also has an apparent growth inhibition effect on algae in certain cases [14], [76].

A prior study has been performed suggesting the possibility of an intermediate microbial treatment process for the $\mathrm{AqAl}$, where a heterotrophic organism would use the organic compounds as carbon and energy sources, thus producing more biomass for the hydrothermal reaction process and potentially detoxifying the $\mathrm{AqAl}$ and remineralizing its nitrogen and phosphorus before refeed back to the algae growth operation [77]. A separate HTL study confirmed that bacterial and fungal biomass slurries could produce biocrude of a similar yield and quality as algae [78] and a separate life-cycle analysis of an algae HTL process with an intermediate microbial growth step could improve the process in areas such as land footprint and reduce the overall cost per volume of biocrude [79]. However, the organisms used in the initial AqAl growth study, Escherichia coli and Pseudomonas putida, experienced significant growth inhibition above $20 \mathrm{vol} \% \mathrm{AqAl}$ in the growth media, and consumed only a small fraction of the organic carbon present [77]. The goal of this study was to improve microbial growth rate and yield through a directed evolution experiment and elucidate the mechanisms of improved growth through genetic resequencing and investigation of individual mutations.

$\mathrm{AqAl}$ is a complicated product that contains diverse array of organic compounds that have not been fully characterized [7], [11]. Several suspected growth-inhibitory classes of compounds, such as phenolics, have been identified in AqAl and similar byproducts, yet their actual toxicity towards algae or microbes has not been proven [14], [16]. Because of the elusive nature of which compounds in AqAl are challenging to microbial growth, or even what exactly the AqAl is comprised of, rational genetic 
engineering of bacterial strains for improved growth would be difficult. We instead attempted to increase microbial tolerance and metabolism through evolution, with AqAl providing the selective environmental pressure while the culture undergoes random mutagenesis and natural selection over many cell division generations. When strains with improved growth are generated from this process, whole genome sequencing technology can then facilitate identification of the mutations they acquired for further study. Adaptive evolution through serial passage of cell cultures and genetic resequencing has proven successful for optimization of substrate metabolism [80] as well as resistance to growthinhibiting compounds [42], but these studies were performed on chemically defined media with known selective factors. Recently, evolution of yeast monocultures has been carried out on whole biomass hydrolysate [27], [41], but these studies do not utilize full genome sequencing for the identification of exact beneficial mutations. This study is the first to our knowledge that studies bacterial monocultures cultures evolved on a complex biomass lysate and also characterizes the mutations generated with full genome sequencing. Our project design is summarized in Figure 4.1. First, a 20 wt\% slurry of Nannochloropsis oculata was processed in a HTL reaction at $350{ }^{\circ} \mathrm{C}$ for one hour. The products were separated and AqAl was isolated. This AqAl was used to make bacterial growth media between 10 and 30 vol\% AqAl as the sole C/N/P source. The organisms used in the initial Nelson et al study, E. coli and P. putida, were grown in three separate 10 vol\% AqAl tube cultures (Lineages) each, and a small amount of each culture was passaged into fresh media every $\sim 5$ generations. The AqAl concentration was increased to 20 vol\% after 31 passages, and 30 vol\% after 54. Evolution was stopped after $\sim 300$ generations after cell passage 60 . Ten isogenic isolates were chosen from each Lineage and screened with cell growth and carbon consumption studies to narrow each group of ten to down to three Strains of Interest. These strains were analyzed with Illumina whole-genome sequencing technology and single nucleotide polymorphisms (SNPs) attained over the course of evolution were identified. A selection of six interesting SNPs in E. coli were individually incorporated into a host E. coli strain and studied for their unique effects on cell growth in AqAl media as well as media with other known growth-affecting factors in order to elucidate the specific mechanisms of their effects. 
The same Illumina sequencing technology that facilitates efficient full genome sequencing also allows for precise quantification of the RNA transcriptome [45]. We grew the top-performing evolved E. coli strain along with its parent strain in both standard minimal medium as well as AqAl medium. RNA samples were isolated from each culture and their full transcriptome was sequenced in order to determine gene expression changes between strain and growth conditions. 


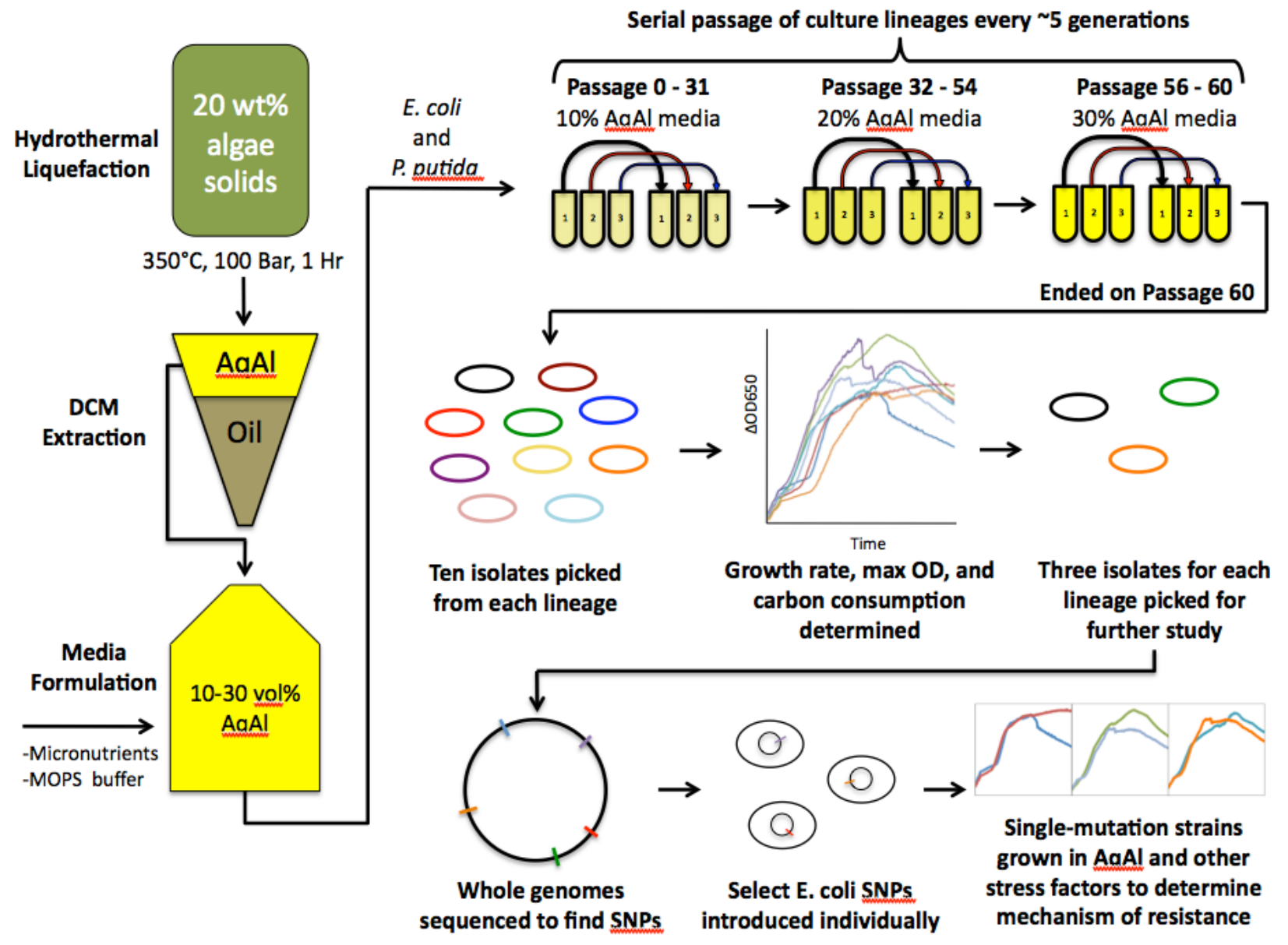

Figure 4.1 Project design 


\subsection{Results}

\subsubsection{Strains of Interest Phenotype}

Ten Sequencing Candidate isolates from each evolution project Lineage were chosen and narrowed down to three Strains of Interest through growth and carbon consumption screening (Table 4.4 in Methods and Materials). Several "E. coli" K12 strains (K1C1 and K1C2) and "P. putida" strains (P1C9, P29, and P3C2) were found to be contaminants of the other species through 16S RNA gene sequencing. However, rather than choosing new strains of the intended species, we chose to continue study of these strains to see if coevolution had any interesting effects on them.

The Strains of Interest were grown in a microplate study using 30 vol\% AqAl medium. Their growth curves are shown in Figure 4.2 and maximum optical densities (Max ODs) and overall growth rates (OGRs) are shown in Figure 4.3.

All evolved P. putida strains showed clear superiority over their parent strain and very similar behavior as each other. All strains seem to share a very similar initial exponential growth phase between four and nine hours, and differ primarily by a second "tapering" growth phase before stationary phase. The parent P. putida strain had a "wavy" growth curve, which is often indicative of flocculation or film formation, which all evolved strains lacked. Evolved strains' Max ODs are very similar, but strain P1C2 clearly has the highest OGR.

E. coli Strains of Interest had more varied performance, with some reaching significantly higher Max ODs at faster OGRs, some reaching similar Max ODs with only slightly faster OGRs (notably, all the contaminant strains), and one (K3C1) actually growing worse than the parent strain in both respects. Of all of the E. coli strains, $\mathrm{K} 2 \mathrm{C} 2$ is clearly the best performing with regards to both Max OD and OGR. 


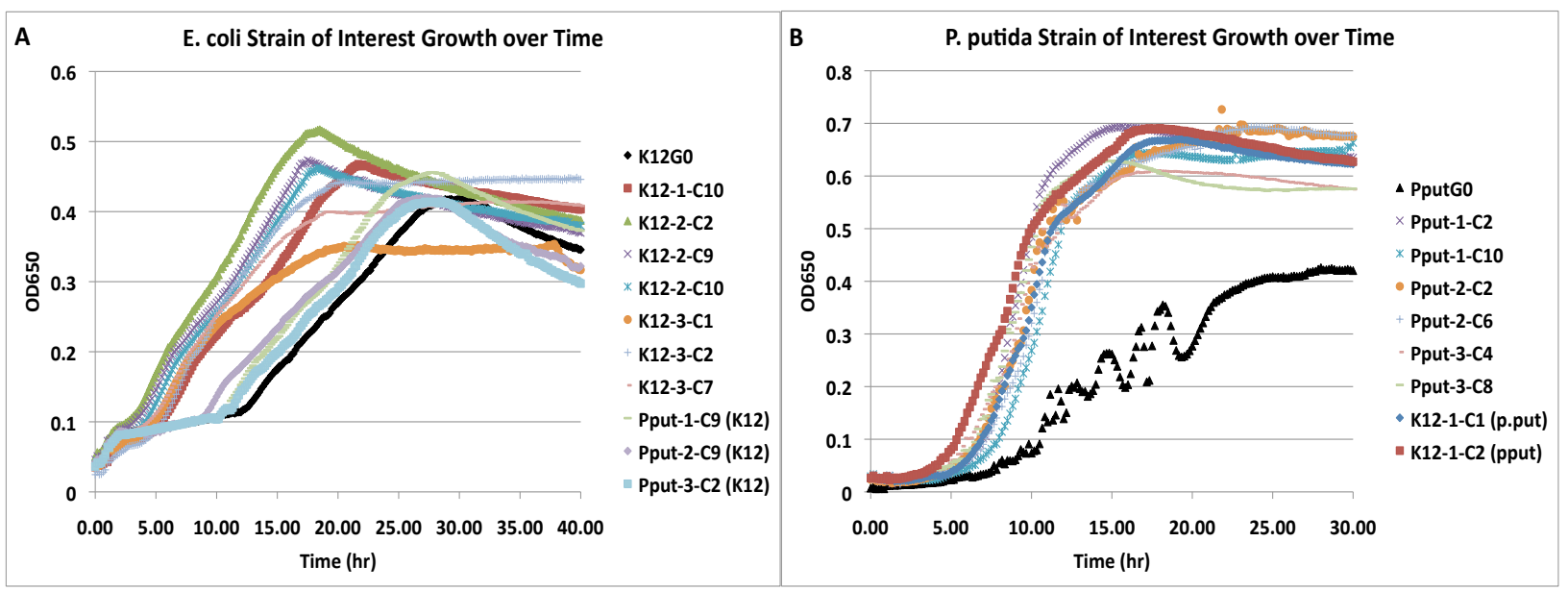

Figure 4.2 E. coli (A) and P. putida (B) Strains of Interest grown in 30 vol\% AqAl Medium. Note that contaminant strains are compared to their appropriate parent strain.

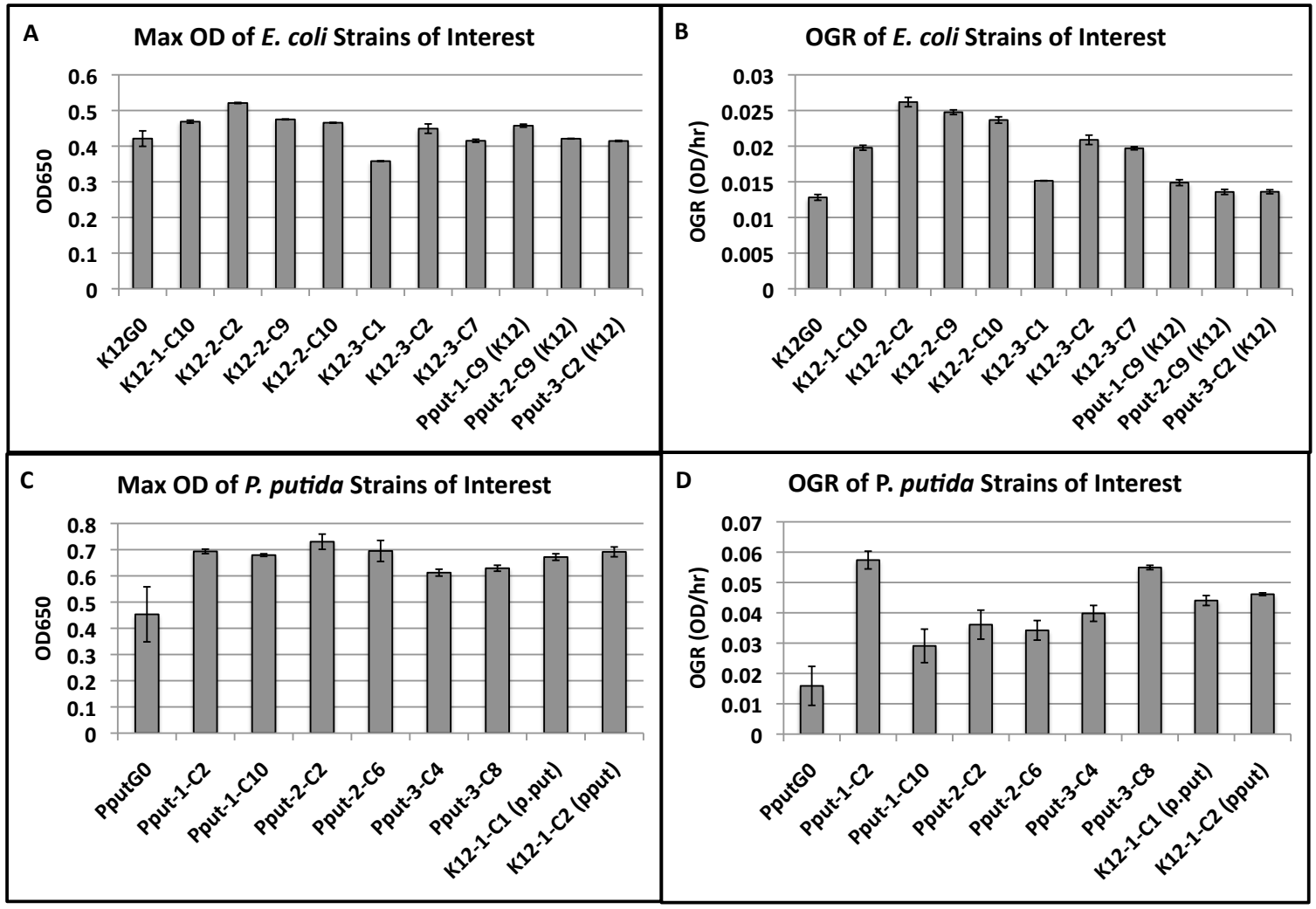

Figure 4.3 Maximum optical densities (A, C) and overall growth rates (B, D) of E. coli and P. putida Strains of Interest when grown in 30 vol\% AqAl. Error bars represent the standard error of the mean of two replicates.

\subsubsection{Full Genome Sequencing}

All Strains of Interest were analyzed with Illumina full genome sequencing technology by the University of Michigan Sequencing Core and the data generated were used to find single nucleotide polymorphisms (SNPs) in these evolved strains. We used an 
in-house alignment software pipeline developed by Minty et al [42] to identify the SNPs and analyzed our parent strains as well to account for any differences our lab strain may have had from the reference genome sequences we used. Figure 4.4 shows results for $P$. putida strains and Figure 4.5 shows results for E. coli strains.

In P. putida several mutations are present across multiple evolution lineages. For example, the "gltA-PP_4195-A" mutation is present in one strain in each lineage. It would be remarkable if the same mutation in the same nucleotide appeared independently across all lineages, but there is also the possibility of cross contamination during culture passaging.

There are six mutations located in intergenic space, indicating a likely regulatory effect, perhaps formation/prevention of a secondary DNA structure or an effect on the binding of a promoter/repressor molecule.

Surprisingly, the strain that performed the best in phenotype characterization (P1C2) had only two mutations, the fewest of any Strain of Interest. However, one of its mutations is unique. It is in the PP_2793 gene, which codes for an acyl-CoA dehydrogenase enzyme. This gene is defined as such through homology with other enzymes and there is no relevant literature at this time investigating its exact function. However, similar acyl-CoA dehydrogenase enzymes in various $P$. putida strains have been found to be key in metabolism of various fatty acids and aromatic compounds [81], [82]. The P1C2 strain may be uniquely able to metabolize complex denatured lipid and protein products present in AqAl. 


\begin{tabular}{|c|c|c|c|c|c|}
\hline Gene Name & Gene Function & Location & WT & Mutant & Residue Change \\
\hline PP_2793 & acyl-CoA dehydrogenase & $\mathbf{3 1 8 2 7 4 4}$ & $\mathbf{C}$ & $\mathbf{G}$ & PRO-136 = > ARG-136 \\
\hline PP_3486 & cytochrome c & 3951158 & $\mathbf{A}$ & $\mathbf{T}$ & ILE-22 => LYS-22 \\
\hline \multirow{3}{*}{ PP_1695 } & \multirow{3}{*}{ integral membrane sensor hybrid histidine kinase } & 1886927 & C & $\mathbf{T}$ & GLY-1115 => SER-1115 \\
\hline & & 1887316 & $\mathbf{A}$ & G & ILE-985 => THR-985 \\
\hline & & 1888040 & $\mathbf{T}$ & $\mathbf{C}$ & SER-744 => GLY-744 \\
\hline \multirow{3}{*}{ gltA - PP_4195 } & \multirow{3}{*}{ (gltA) type II citrate synthase : hypothetical protein } & 4741121 & $\mathbf{A}$ & $\mathbf{T}$ & - \\
\hline & & 4741135 & $\mathbf{G}$ & $\mathbf{T}$ & - \\
\hline & & 4741365 & C & $\mathbf{T}$ & - \\
\hline \multirow{2}{*}{ PP_1488 } & \multirow{2}{*}{ methyl-accepting chemotaxis sensory transducer } & 1692767 & G & $\mathbf{A}$ & ALA-290 => THR-290 \\
\hline & & 1692775 & $\mathbf{G}$ & $\mathbf{A}$ & GLU-292 => GLU-292 \\
\hline PP_1690 - PP_1691 & hypothetical proteins & 1883248 & $\mathbf{T}$ & $\mathbf{C}$ & - \\
\hline PP_1709 - PP_1710 & $\begin{array}{c}\text { fumarylacetoacetate hydrolase : major facilitator } \\
\text { superfamily transporter phthalate permease }\end{array}$ & 1909387 & $\mathbf{T}$ & C & - \\
\hline PP_4929 - PP_4930 & $\begin{array}{l}\text { LysR family transcriptional regulator : small } \\
\text { multidrug resistance protein }\end{array}$ & 5610571 & G & $\mathbf{T}$ & - \\
\hline PP_0264 & sensor histidine kinase & 318616 & $\mathbf{G}$ & $\mathbf{A}$ & SER-375 => LEU-375 \\
\hline PP_2729 & hypothetical protein & 3114367 & $\mathbf{G}$ & $\mathbf{A}$ & ARG-224 => HIS-224 \\
\hline PP_4929 & LysR family transcriptional regulator & 5610082 & G & $\mathbf{T}$ & ALA-143 => GLU-143 \\
\hline PP_2554 & 4-hydroxyphenylpyruvate dioxygenase & 2901458 & $\mathbf{C}$ & G & ASP-181 => GLU-181 \\
\hline
\end{tabular}

\begin{tabular}{|c|c|c|c|c|c|c|c|c|}
\hline Gene Nam & P1C2 & P1C10 & P2C2 & P2C6 & P3C4 & P3C8 & K1C1 & K1C2 \\
\hline \multicolumn{9}{|l|}{ PP_2793 } \\
\hline \multicolumn{9}{|l|}{ PP_3486 } \\
\hline \multicolumn{9}{|l|}{ PP_1695-A } \\
\hline \multicolumn{9}{|l|}{ PP_1695-B } \\
\hline \multicolumn{9}{|c|}{ PP_1695-C } \\
\hline \multicolumn{9}{|c|}{ gltA - PP_4195-A } \\
\hline \multicolumn{9}{|c|}{ gltA - PP_4195-B } \\
\hline \multicolumn{9}{|c|}{ gltA - PP_4195-C } \\
\hline \multicolumn{9}{|l|}{ PP_1488-A } \\
\hline \multicolumn{9}{|l|}{ PP_1488-B } \\
\hline \multicolumn{9}{|c|}{ PP_1690 - PP_1691 } \\
\hline \multicolumn{9}{|c|}{ PP_1709 - PP_1710 } \\
\hline \multicolumn{9}{|c|}{ PP_4929 - PP_4930 } \\
\hline \multicolumn{9}{|c|}{ PP_0264 } \\
\hline \multicolumn{9}{|l|}{ PP_2729 } \\
\hline PP_4929 & & & & & & & & \\
\hline PP_2554 & & & & & & & & \\
\hline
\end{tabular}

Figure 4.4 P. putida SNP identification. Top contains gene identity and function, and location of mutation in the genome. Hyphens in between gene names indicate mutations in space between genes. Bottom shows what mutation each strain contains (-A/-B/-C used to indicate different mutations in same gene).

In E. coli there are no identical cross-lineage mutations, which means contamination among E. coli lineages was less likely. There are many mutations shared by strains within Lineage Two or Three, indicating common ancestry with an intermediate strain. The topperforming E. coli strain, $\mathrm{K} 2 \mathrm{C} 2$, contained the largest total amount of mutations (unlike the top P. putida strain, which had the least). It shared three SNPs with the other two Lineage Two strains (which were the second and third best performing Strains of Interest) and had two unique mutations of its own, one in a gene with an unknown product, and one in a 
phosphotransferase system (PTS) gene. No mutations were identified in P2C9, one of the contaminant strains.

We performed PCR on the suspected mutation sites in each E. coli Strain of Interest and confirmed the mutation was present via Sanger sequencing of the product formed. We did the same thing to each possible mutation site on the parent E. coli K12 strain to confirm the absence of the mutations before the evolution project.

\begin{tabular}{|c|c|c|c|c|c|c|c|c|c|c|c|c|c|}
\hline \multicolumn{2}{|c|}{ Gene Name } & \multicolumn{5}{|c|}{ Gene Function } & \multicolumn{2}{|c|}{ Location } & WT & Mutant & \multicolumn{3}{|c|}{ Residue Change } \\
\hline \multirow{2}{*}{\multicolumn{2}{|c|}{ polB }} & \multicolumn{5}{|c|}{ DNA polymerase II } & \multicolumn{2}{|c|}{65318} & $\mathbf{T}$ & $\mathrm{C}$ & \multicolumn{3}{|c|}{ ILE-15S => VAL-15S } \\
\hline & & \multicolumn{5}{|c|}{ Acetolactate Synthase Subunit } & \multicolumn{2}{|c|}{85999} & $\mathbf{C}$ & $\mathbf{T}$ & \multicolumn{3}{|c|}{ PRO-124 => SER-124 } \\
\hline \multicolumn{2}{|l|}{ ilvH } & \multicolumn{5}{|c|}{ Acetolactate Synthase Subunit } & \multicolumn{2}{|c|}{87397} & $\mathbf{G}$ & $\mathbf{A}$ & \multicolumn{3}{|c|}{ GLY-14 => ASP-14 } \\
\hline \multicolumn{2}{|l|}{ ilvN } & \multicolumn{5}{|c|}{ Acetolactate Synthase Subunit } & \multicolumn{2}{|c|}{3851043} & $\mathbf{T}$ & C & \multicolumn{3}{|c|}{ ASN-17 $=>$ SER-17 } \\
\hline \multicolumn{2}{|l|}{ sapD } & \multicolumn{5}{|c|}{ Antimicrobial Peptide Transport } & \multicolumn{2}{|c|}{1352926} & $\mathbf{C}$ & $\mathbf{T}$ & \multicolumn{3}{|c|}{ GLY-235 $=>$ SER-235 } \\
\hline \multicolumn{2}{|l|}{$\operatorname{sapA}$} & \multicolumn{5}{|c|}{ Antimicrobial Peptide Transport } & 13 & 6347 & $\mathbf{C}$ & $\mathbf{A}$ & GL & $255=7$ & L-25S \\
\hline $\operatorname{manY}$ & & $\mathrm{Ma}$ & inose-s & cific PT & Compon & & 19 & 3524 & C & $\mathbf{T}$ & AL & $148=>$ & $L-148$ \\
\hline yejA & & & Micre & C Tran & orter & & 22 & 2940 & $\mathbf{T}$ & A & TY & $193=$ & $\mathrm{N}-193$ \\
\hline & & & & & & & 27 & 0457 & C & $\mathbf{T}$ & GLI & $169=>$ & $p-169$ \\
\hline pka & & Pr & tein Ly & e Acety & ransfera & & 27 & 1067 & C & $\mathbf{T}$ & $\mathrm{SE}$ & $372=$ & $U-372$ \\
\hline prov & & Glyc & ne Bet: & Trans & rter Sub & & 28 & 5823 & $\mathbf{C}$ & $\mathbf{T}$ & GLI & $337 \Rightarrow$ & $p-337$ \\
\hline ptsP & & & Fu: & PTS En & me & & 29 & 6838 & $\mathbf{C}$ & A & GLL & $333=>$ & $p-533$ \\
\hline yhhH & & & Hyp & etical $\mathrm{F}$ & tein & & 36 & 3658 & C & A & & $-87=$ & $N-87$ \\
\hline & & & & & & & 41 & 6935 & C & A & & $-96=>$ & $N=96$ \\
\hline glpK & & & & erol Kin & & & 41 & 7061 & C & A & & $-54=?$ & S-54 \\
\hline & Line & age 1 & & ineage : & & & & Lineage & & & & ntamin: & \\
\hline Gene Name & $\mathrm{K}:$ & C10 & $\mathrm{K} 2 \mathrm{C} 2$ & $\mathrm{~K} 2 \mathrm{C} 9$ & $\mathrm{~K} 2 \mathrm{C} 10$ & K3 & & $\mathrm{K} 3 \mathrm{C} 2$ & & $\mathrm{~K} 3 \mathrm{C7}$ & P1C9 & P2C9 & P3C2 \\
\hline poeds & & & & & & & & & & & & & \\
\hline ilvI & & & & & & & & & & & & & \\
\hline invet & & & & & & & & & & & & & \\
\hline inN & & & & & & & & & & & & & \\
\hline $\operatorname{sapo}$ & & & & & & & & & & & & & \\
\hline $\operatorname{saph}$ & & & & & & & & & & & & & \\
\hline $\operatorname{manY}$ & & & & & & & & & & & & & \\
\hline$x \in A$ & & & & & & & & & & & & & \\
\hline plata-A & & & & & & & & & & & & & \\
\hline plaa-b & & & & & & & & & & & & & \\
\hline $\operatorname{sen} 2$ & & & & & & & & & & & & & \\
\hline pespe & & & & & & & & & & & & & \\
\hline ginht & & & & & & & & & & & & & \\
\hline glpK-A & & & & & & & & & & & & & \\
\hline gipk-8 & & & & & & & & & & & & & \\
\hline zrea & & & & & & & & & & & & & \\
\hline
\end{tabular}

Figure 4.5 E. coli SNP identification. Top contains gene identity and function, and location of mutation in the genome. Bottom shows what mutation each strain contains (-A/-B used to indicate different mutations in same gene). 


\subsubsection{Introduction of Individual SNPs through Homologous Recombination}

With all mutations confirmed, we chose three specific mutation classes to focus on for further study: the two glpK SNPs, the ptsP SNP, and the SNPs in the ilv family of genes. Our goal was to introduce these SNPs individually to a lab strain of E. coli and measure their effects on cell growth in both AqAl media as well as various media designed to shed light on their mechanism of action.

The $g l p K$ gene codes for glycerol kinase, an enzyme in the glycerol metabolism pathway. A previous serial-passaging evolution study on E. coli using glycerol as the only organic carbon source in the minimal media produced evolved strains with mutations in glpK [80]. These strains could grow faster on glycerol, though not necessarily more efficiently. Since glycerol is a known component in AqAl [77] it is likely that the mutations in our evolved strains improve its metabolism.

The $p t s P$ gene is suspected to be involved with the coordination of carbon and nitrogen assimilation [83]. AqAl is known to have a carbon/nitrogen ratio much lower than ideal for microbial growth [77] so the SNP identified in this gene (causing a premature stop in transcription and likely deactivating it) may help the strain better handle this.

The ilvH, ilvN, and ilvI genes all code for a subunit of the acetolactate synthase enzyme, a vital enzyme in the production of essential branched-chain amino acids. A SNP in one of these genes was found in every E. coli Strain of Interest in every Lineage (though not in contaminants), indicating its likely importance in AqAl tolerance. Acetolactate synthase enzymes in plants are the target of various classes of herbicides, including imidizolinones. Several of these cyclic nitrogen-containing compounds have been found in AqAl by members of the Philip Savage Lab at University of Michigan (publication pending), so the SNPs may be interfering with the inactivation of the enzyme by these compounds. Indeed, plant species have been made resistant to these compounds by single mutations in their own acetolactate synthase genes [84]. 
All six of these mutations ( $g l p K A, g l p K B, p s t P$, ilvH, ilvN, and ilvI) were encoded on 90bp single-stranded DNA and introduced to lab strain E. coli EcHW24 via electroporation. This strain contains a defective mutS gene as well as " $\lambda$-Red" phage-derived genes that make it susceptible to incorporation of partially homologous oligomers on the lagging strand of the DNA replication fork. Efficiency with this method is high enough that we did not need to use selection factors. We screened isolates from post-electroporation cultures with allele-specific PCR, using primers designed to only yield products from templates containing the desired SNP, then confirmed the results with Sanger sequencing. In this way, we successfully produced EcHW24 strains with the six individual SNPs, which will be henceforth referred as strains "E. coli [name of mutated gene]".

\subsubsection{Growth of Single SNP Strains in AqAl and Minimal Media}

The new single SNP strains were all grown in $30 \mathrm{vol} \% \mathrm{AqAl}$ as well as Complete MOPS minimal media in a plate study similar to the one used on the original Strains of Interest. The Complete MOPS medium (with glucose, nitrogen, and phosphorus as the $\mathrm{C} / \mathrm{N} / \mathrm{P}$ sources instead of AqAl) was included to see if the mutations' effects were specific to the unique properties of AqAl. Figure 4.6 shows growth curves over time and Figure 4.7 shows Max OD and OGR data.

The ilv mutations appear to confer the best effect on growth in AqAl, producing the highest Max ODs and OGRs. The ptsP mutation also has a positive effect in AqAl, with a slight Max OD increase but a significant OGR increase. The $g l p K$ mutations produce almost identical Max ODs and OGRs as the parent strain. However, their growth curves show that they initially grow much faster than the parent strain during the initial phases of diauxic growth, yet they slow during the very last stage and the parent strain "catches up", with all three strains reaching similar Max ODs at similar total times. This gives them each similar overall growth rates in AqAl media.

In Complete MOPS media, most strains grew almost identically to the parent, showing that their benefits in AqAl media likely address some specific property of AqAl and not just general cell health and growth. The glpKB mutant showed slightly better than normal growth in Complete MOPS media, while ilvN showed a decrease. This is contrary to 
their effects in AqAl media, where glpKB showed no overall advantage and ilvN conferred an advantage.
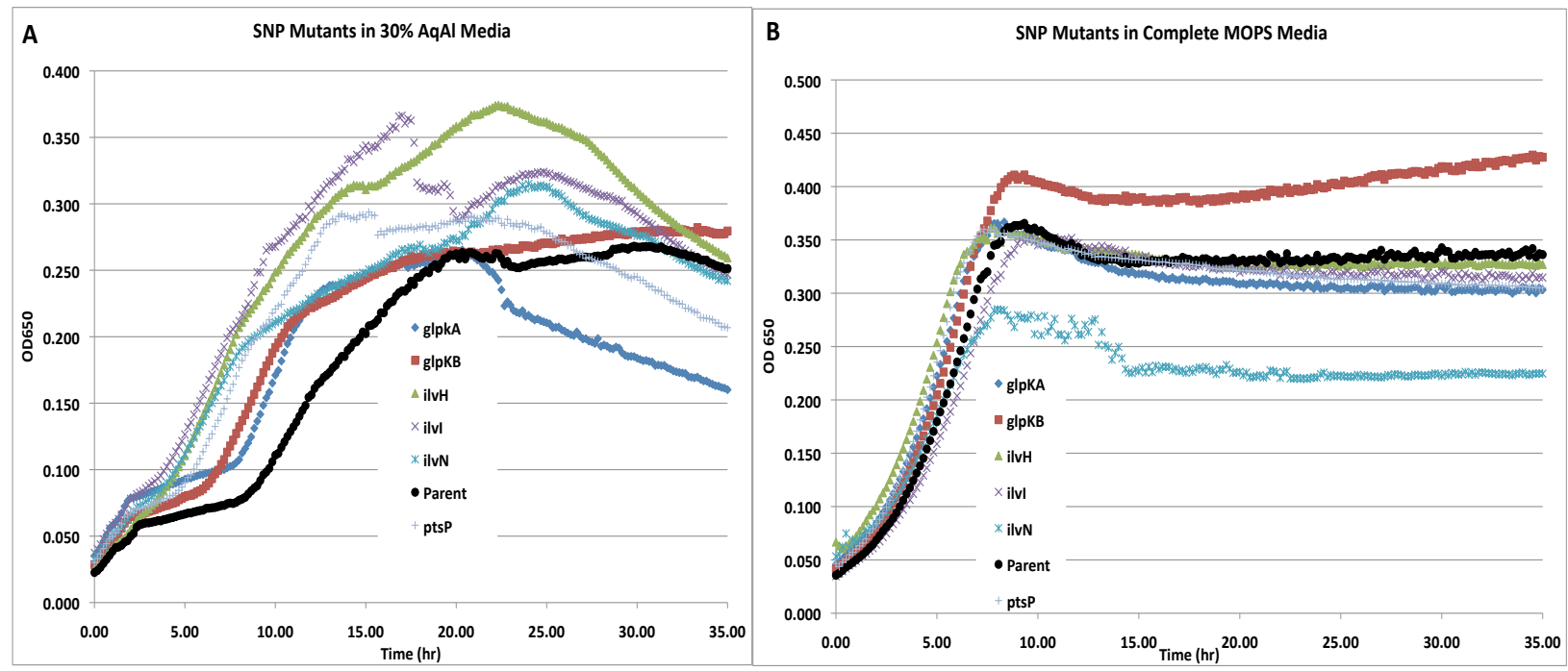

Figure 4.6 Growth data for single-SNP strains and parent EcHW24 strain (black) grown in 30 vol\% AqAl media (A) and Complete MOPS media (B). Each data point is the average of three replicates.

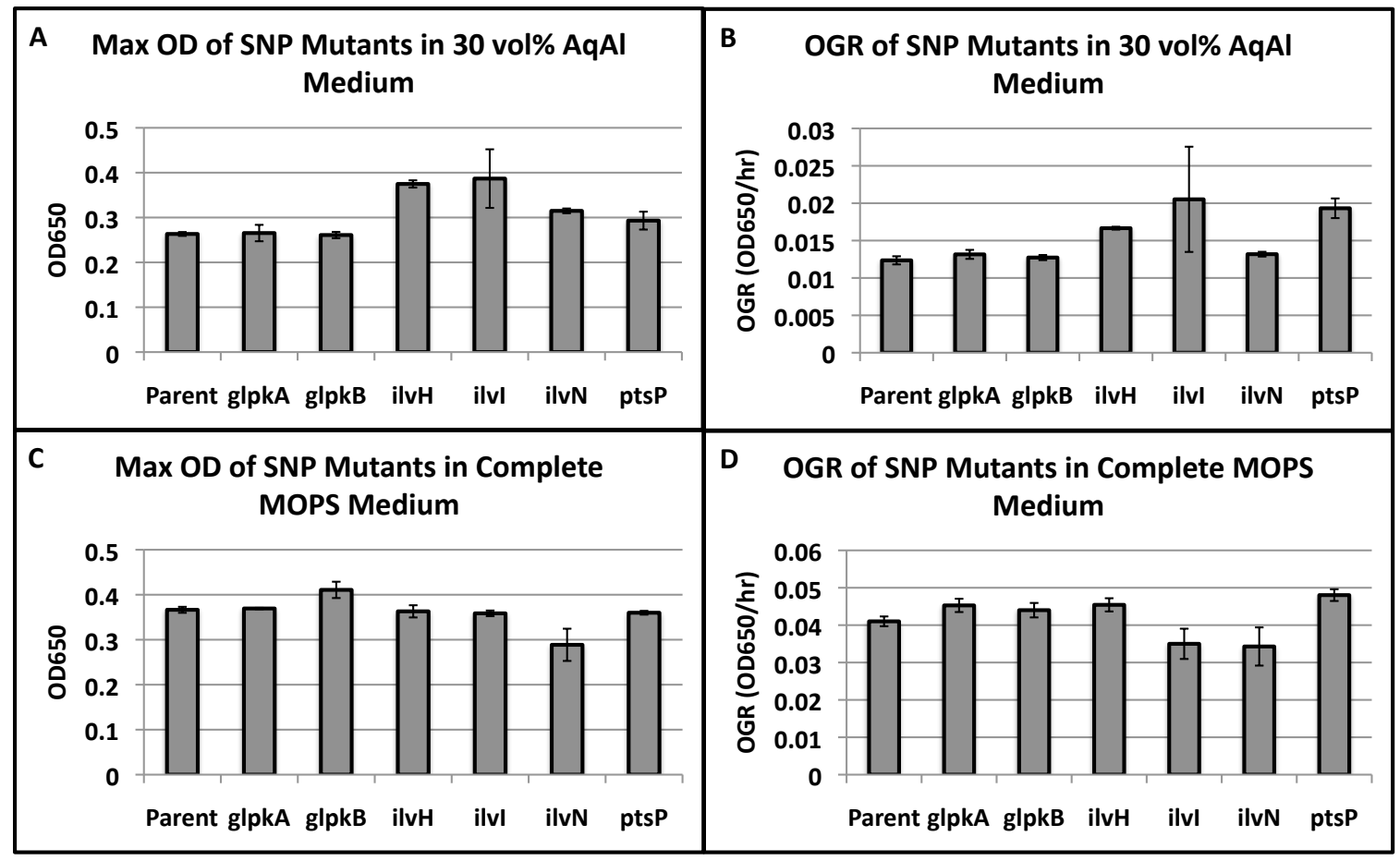

Figure 4.7 Max OD (A, C) and OGR (B, D) of single-SNP mutant strans and parent EcHW24 strain grown in 30 vol\% AqAl Media and Complete MOPS media. Error bars represent standard deviation of three replicates. 


\subsubsection{Growth of SNP Mutants in Special Media}

The single SNP mutant strains were grown along with their EcHW24 parent strain in various versions of minimal MOPS media designed to elucidate the mechanism of the mutations' effect on AqAl tolerance. GlpK mutants were grown in media with glycerol, the $p t s P$ mutant was grown in media with varying $\mathrm{C} / \mathrm{N}$ ratios, and the ilv mutants were grown in media containing acetolactate synthase inhibitors.

\subsubsection{GlpK Mutants Growth in Glycerol Media}

The two strains with a SNP in the glpK gene (referred to as $g \operatorname{lp} K A$ and $g \operatorname{lp} K B$ ) as well as their parent strain were grown in a microplate study where varying combinations of glycerol and glucose were used as the carbon sources in MOPS minimal media. 0 shows growth curves and Figure 4.8 shows Max ODs and OGRs. The glpKB slightly outperformed the parent strain in both Max OD and OGR at all concentrations containing at least some glucose, while the $\operatorname{glpKA}$ strain performed very similarly to the parent in partial-glucose media. But there is a striking reduction in parent strain growth rate in glycerol-only media that both $g l p K$ strains do not experience. The parent does eventually get to a similar Max OD in this situation, though.

The glycerol kinase encoded by the $g l p K$ gene accomplishes an important initial step in the metabolism of glycerol (transfer of a phosphoryl group to make glycerol-3phosphate). It exists as an active dimer or inactive tetramer and glycolytic pathway intermediate fructose 1,6-bisphosphate (FBP) and sugar phosphotransferase system phosphocarrier protein IIAGlc both allosterically inhibit glycerol kinase by stabilizing the inactive tetramer form or binding to a site on the dimer and inducing a conformational change, respectively [85]. These two compounds increase in concentration within the cell during the metabolism of other non-glycerol substrates (including glucose), so wild type $E$. coli is naturally configured to repress glycerol metabolism when more favored substrates are present. Other mutations in glpK discovered by the Palsson group relieved this inhibition in the presence of both glycerol and glucose [86]. This is likely the method of action our unique mutations, since our glucose/glycerol mix phenotype results are quite similar to theirs. 


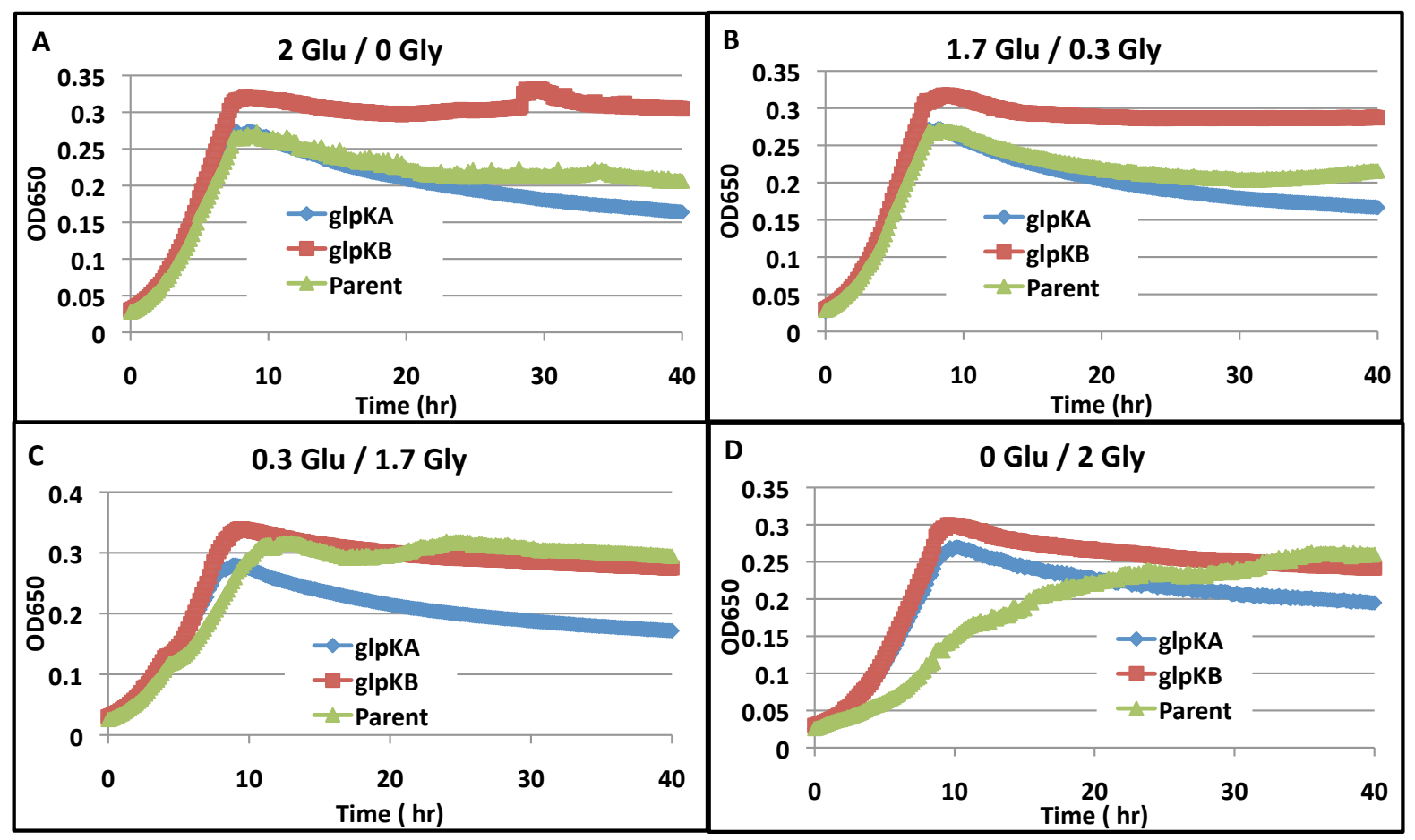

Figure 4.8 Growth of $g l p K$ mutants and parent strain in MOPS minimal media containing different concentrations of glucose (Glu) and Glycerol (Gly), from $2.0 \mathrm{~g} / \mathrm{L}$ Glu and $0 \mathrm{~g} / \mathrm{L}$ Gly (A) to $0 \mathrm{~g} / \mathrm{L} \mathrm{Glu}$ and $2.0 \mathrm{~g} / \mathrm{L}$ Gly (B). Each data point is the average of three replicates.

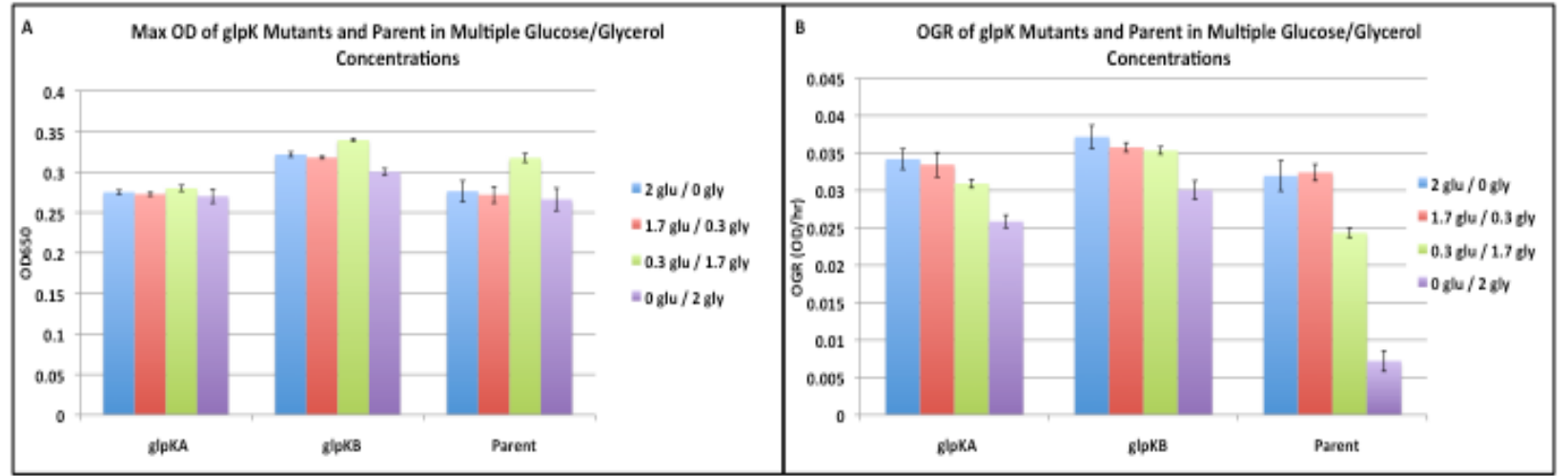

Figure 4.9 Max OD (A) and Overall Growth Rate (B) of glpK mutants and parent strain in MOPS minimal media containing various concentrations of glucose and glycerol. Error bars represent one standard deviation or three replicates 


\subsubsection{PtsP Mutant growth in Varying $C / N$ ratio Media}

The ptsP mutant was grown in a microplate experiment alongside of the parent strain in media with a set glucose content of $2 \mathrm{~g} / \mathrm{L}$ and varying ammonia content to make the C:N ratio (by weight) 0.2 to 3.0. The C:N ratio in AqAl itself is $\sim 1.5: 1$ [77]. This range was chosen to include twice the $\mathrm{C}: \mathrm{N}$ of $\mathrm{AqAl}$, all the way down to a level containing so much nitrogen that the ammonia concentration starts to become independently toxic. Figure 4.10 shows the growth curves of the microplate experiment and Figure 4.11 shows the Max OD and OGR data. Both strains had similar Max ODs at each condition, with smaller values at the lowest C:N ratio likely due to inhibitory ammonia levels. The $p s t P$ mutant outperforms the parent in OGR at all C:N levels. However, the difference between the OGR of both strains remains consistent at each C:N ratio. These Max OD and OGR behaviors are similar to the comparison of the two strains in AqAl media (Figure 4.6), but since there is little change in performance across varying C:N ratios, it is unclear whether the $p t s P$ mutation's method of action is related to the regulation of carbon and nitrogen uptake. 


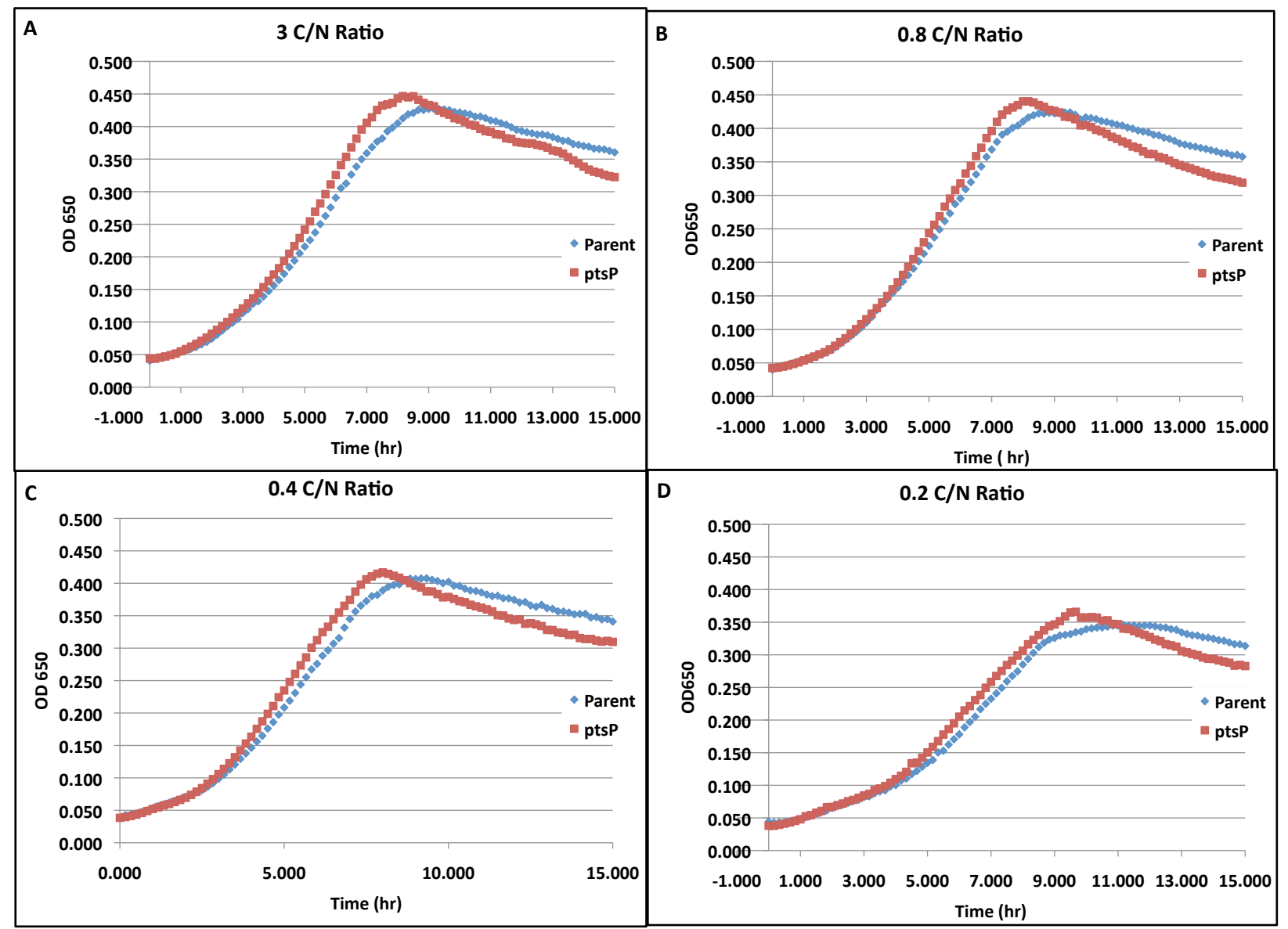

Figure 4.10 Growth of PtsP and parent strain in media with varying C:N ratios. Each data point is the average of three replicates.

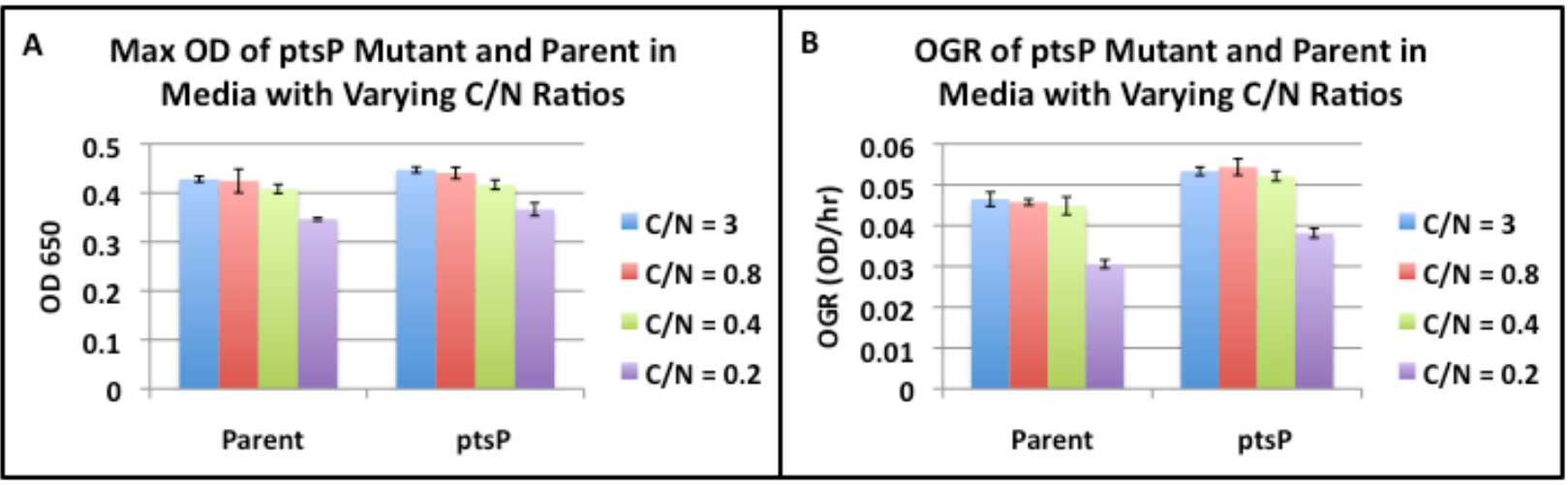

Figure 4.11 Maximum optical density (A) and OGR (B) of ptsP mutant and parent strains growing in MOPS minimal media with varying $\mathrm{C}: \mathrm{N}$ ratios. Error bars represent the standard deviation of three replicates. 


\subsubsection{Ilv Mutants growth in Acetolactate Synthase Inhibitor Media}

We investigated the effects of known acetolactate synthase inhibitors (in plants) suspected to be present in AqAl. The cyclic imidazolinone-class compounds imazaquin, imazapyr, and imazamethabenz were first added to Complete MOPS Media over a wide range of concentrations to give us a general idea of when they started to become inhibitory to wild type E. coli growth. Then the compounds were added at varying concentrations around these toxicity levels and ilvI, ilvH, and ilvN strains, as well as the EcHW24 parent, were grown in multiple microplate tests.

IlvN consistently grew worse in all media, even Complete MOPS with no inhibitors added, when compared to the parent strain (data not shown). This is consistent with its performance in our initial Complete MOPS growth tests (Figure 4.6B) but does little to explain its improved growth rate in actual AqAl (Figure 4.6A). Tests in imazapyr and imazamethabenz proved inconclusive as well, with all ilvH and ilvI strains showing very similar performance to the parent strain as inhibitor concentration increased (data not shown).

Tests with imazaquin at $100 \mathrm{ppm}$ and $500 \mathrm{ppm}$, and Complete MOPS as a control, produced some promising results. Figure 4.12 shows the growth curves and Figure 4.13 shows the Max OD and OGR data. Both ilv strains are mostly unaffected across the both levels of imazaquin, while the parent strain has a decrease in both Max OD and OGR. However, the parent strain starts out at lower performance when compared to the mutants in the control medium, and high variability in culture growth (common when using growthinhibiting compounds) makes it hard to conclude if the mutants are indeed outperforming the parent based on imazaquin resistance. 

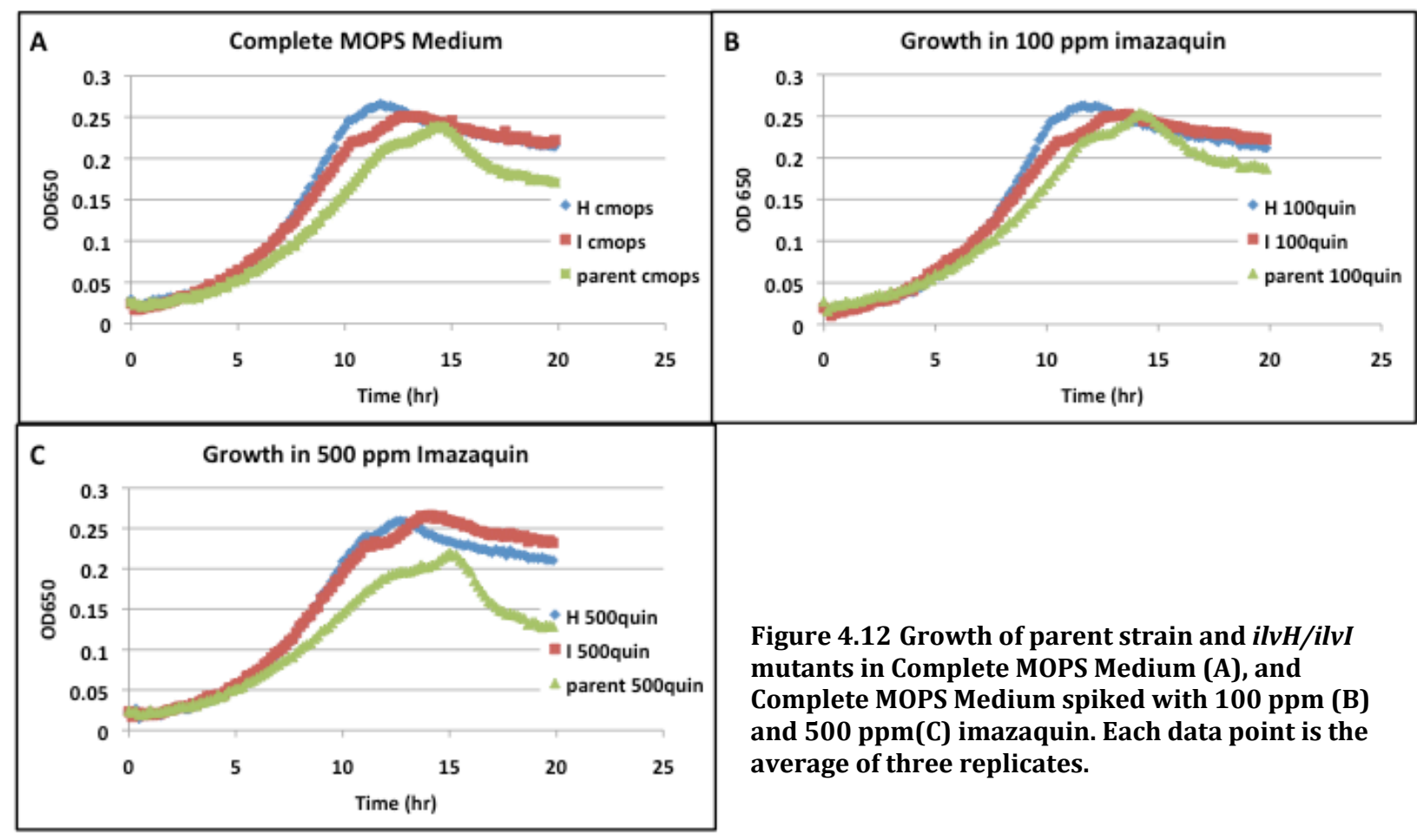

Figure 4.12 Growth of parent strain and ilvH/ilvI mutants in Complete MOPS Medium (A), and Complete MOPS Medium spiked with 100 ppm (B) and 500 ppm(C) imazaquin. Each data point is the average of three replicates.

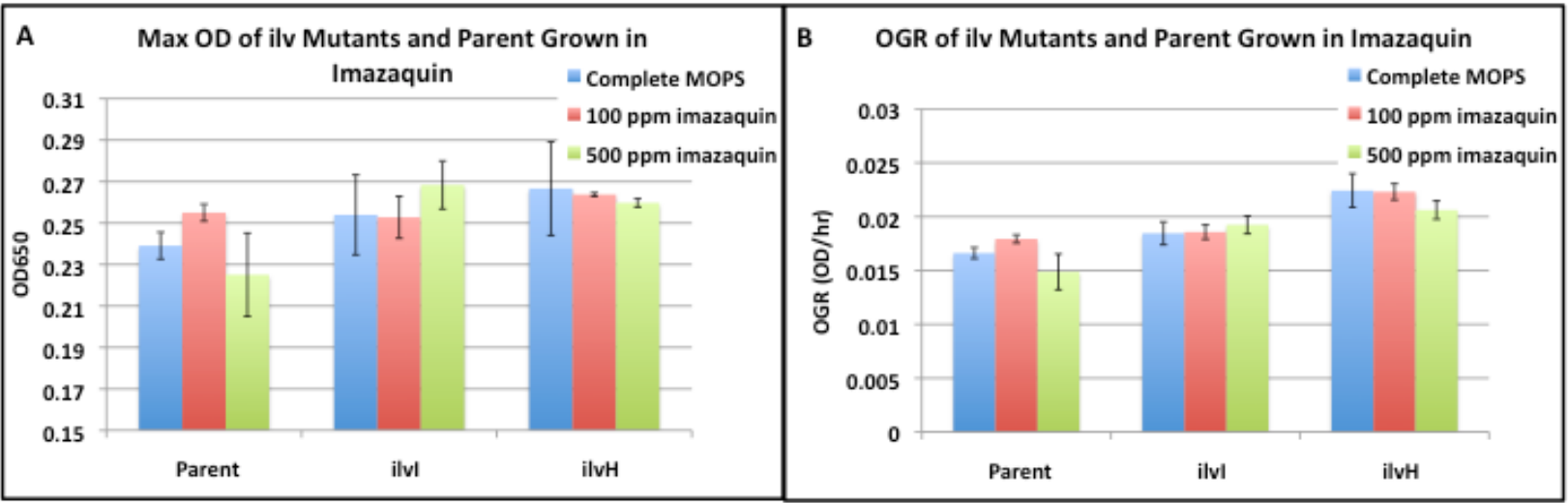

Figure 4.13 Maximum ODs (A) and Overall Growth Rates (B) of ilv mutants and parent strain grown in imazaquinspiked media. Error bars are standard deviations of three replicates. 


\subsubsection{Transcriptome analysis}

In addition to the genetic comparison of several evolved E. coli and P. putida strains, we chose the one particular evolved E. coli strain (K2C2) to compare to its parent strain in terms of their RNA transcriptomes.

We grew parent E. coli K12 and evolved E. coli K2C2 in both Complete MOPS and 30 vol\% AqAl media. We harvested cultures in mid-log growth phase, extracted RNA, depleted ribosomal RNA (a large fraction of total bacterial RNA that has little to do with transcription rates), and sent the RNA to the University of Michigan Sequencing Core. The Sequencing Core converted the RNA to cDNA libraries and analyzed the samples with the same Illumina sequencing technology as we previously utilized for full genome sequencing. The data were sent to the University of Michigan Bioinformatics Core, which performed full transcriptome alignment and gene expression quantification. They then determined differential gene expression between both organisms in the same media (K12 and K2C2 in Complete MOPS medium, K12 and K2C2 in AqAl medium) as well as the same organism in differing media (K12 in both Complete MOPS and AqAl media, K2C2 in both Complete MOPS and AqAl media), for a total of four differential comparisons.

\subsubsection{Differential Expression of Individual Genes}

Figure 4.14 summarizes the total number of differentially expressed (DE) genes identified in the study. Figure 4.14a shows a Venn diagram of DE genes between the mutant (Mut) and parent (WT) strain in both Complete MOPS and 30 vol\% AqAl media, with the intersection representing genes differentially expressed in both media. Roughly 250 unique genes are DE in each media, with $70 \mathrm{DE}$ in both media. Figure $4.14 \mathrm{~b}$ shows DE genes of each strain compared to themselves between Complete MOPS and AqAl media growth conditions. Both strains share a large set of 2120 DE genes between Complete MOPS and AqAl, showing much of the parent's reaction to AqAl is conserved after evolution. The parent also has a unique set of $342 \mathrm{DE}$ genes in the different media while the mutant has 288. This shows that the mutant ceased roughly 300 reactions to AqAl and gained roughly 300 new ones when compared to the parent. 


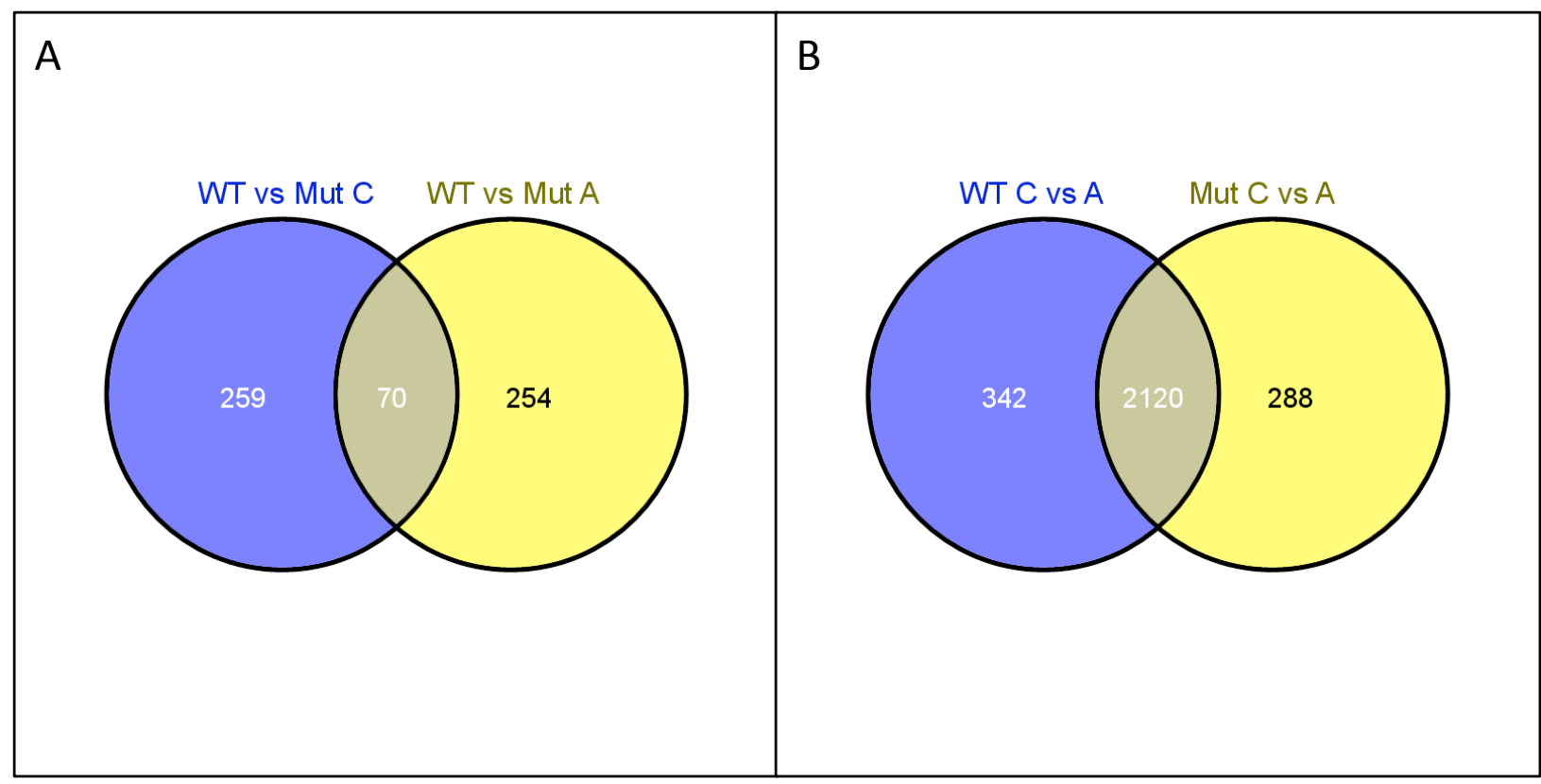

Figure 4.14 Number of unique and shared differentially expressed genes in different comparisons. " $A$ " shows differential expression between parent (WT) and mutant (Mut) in both Complete MOPS (C) and 30 vol\% AqAl (A). "B" shows differential expression between the two media types for both parent and mutant.

Table 4.1 lists the most differentially expressed genes in terms of the magnitude of difference between mutant and parent transcription when grown in 30 vol\% AqAl medium. Two lists of ten each show the "top ten" genes with highest over-expression in the mutant as well as the "bottom ten" with lowest under-expression when compared to the parent. The mutant over-expresses genes involved in acid stress response, particularly ones relating to glutamate/glutamic acid metabolism. One gene, hyaA plays a role in nitrotoluene degredation, a problematic xenobiotic compound. The mutant appears to mainly downregulate genes involved with the synthesis of biotin and specific amino acids. 
Table 4.1 The most differentially expressed genes in the mutant when grown in 30 vol\% AqAl medium (in terms of fold change from parent). Expression is measured in Fpkm, fragments of gene per kilobase of gene transcript per million total mapped reads.

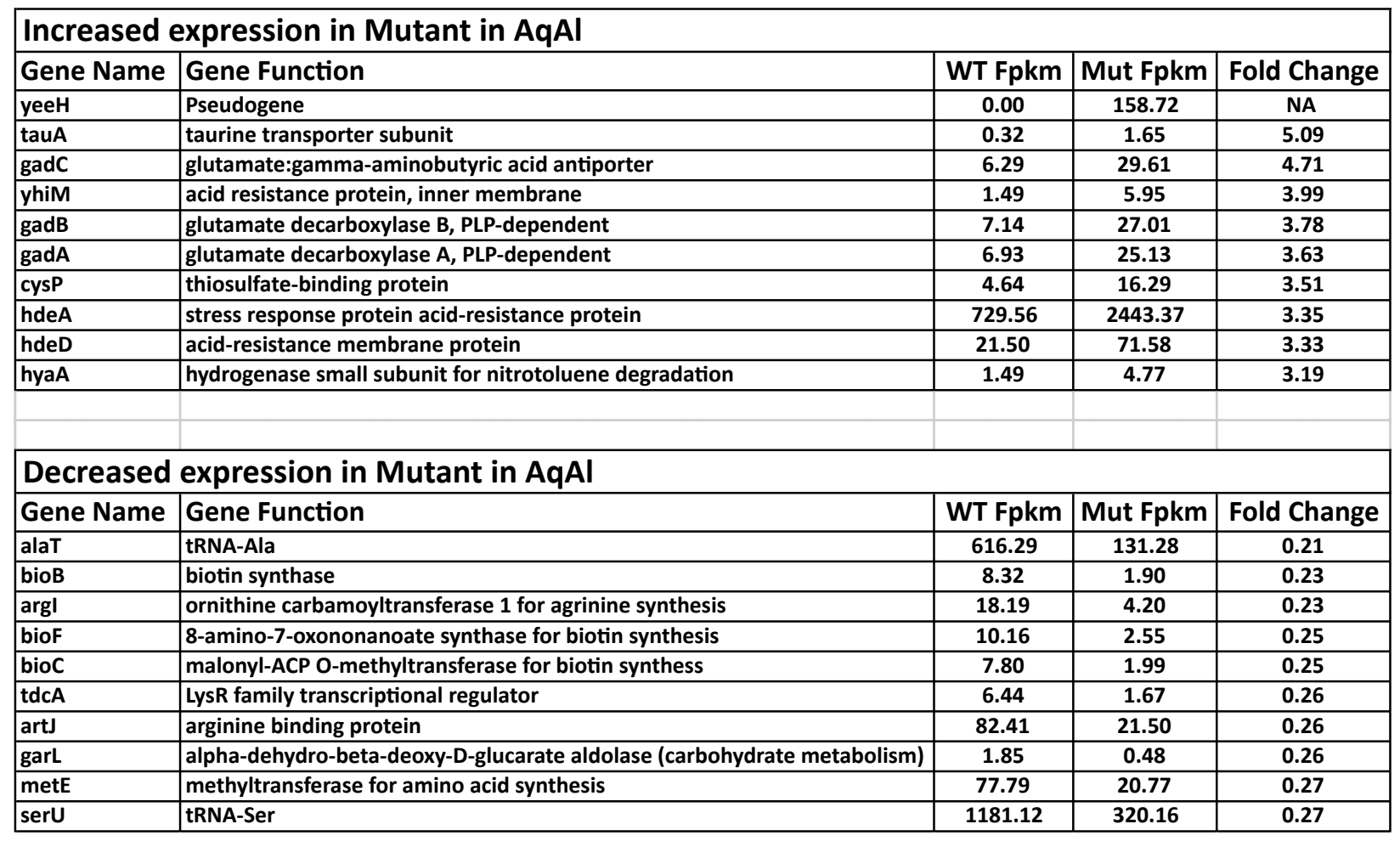




\subsubsection{Differential Expression of Pathways}

Examining small "top ten" lists is only a cursory analysis of the large data set generated in the transcriptome study. Table 4.1 presents only 20 genes identified in one DE gene comparison, when there are actually four comparison data sets with over 2000 genes identified. To make these data more manageable, we analyzed it in terms of metabolic pathway representation rather than individual gene expression. We took the lists of differentially expressed genes in each comparison and split them into lists of overexpressed and underexpressed genes. We then used the EcoCyc E. coli genome and pathway database and enrichment tools to determine metabolic pathways that were statistically overrepresented in each list.

The most important comparison to make in this project was which metabolic pathways were differentially expressed between strains K12 and K2C2 when grown in 30 vol\% AqAl medium. This is shown in Table 4.2, with highlighted cells indicating which pathways were also differentially expressed between the two strains in Complete MOPS medium. The specific genes differentially expressed are also shown. Another interesting comparison is the differentially expressed pathways in K2C2 when grown in 30\% AqAl medium opposed to Complete MOPS medium. This is shown in Table 4.3, with highlighted cells indicating pathways also differentially expressed in K12 between the two medium types. 
Table 4.2 Metabolic pathways with higher expression (top) and lower expression (bottom) in K2C2 when compared to the parent K12 strain when grown in 30 vol\% AqAl media. Highlighted entries indicate pathways that were also differentially expressed between K2C2 and K12 when grown in Complete MOPS medium.

\begin{tabular}{|c|c|c|c|c|c|c|c|c|}
\hline Pathways with Higher Expression in K2C2 in AqAl Medium & & & & rentia & expres & gene & & \\
\hline Acid Resistance & gadB & gadA & & & & & & \\
\hline Activation/Inactivation/Interconversion & cysC & cysN & cysD & & & & & \\
\hline Amino Acids Biosynthesis & ilvC & leuD & leuc & leuA & leuB & cysk & & \\
\hline Detoxification & hchA & grxB & gadB & gadA & katE & & & \\
\hline Generation of Precursor Metabolites and Energy & fbaB & poxB & appC & narv & narz & nary & tktB & talA \\
\hline Inorganic Nutrients Metabolism & narV & narz & narY & cysC & cysN & cysD & cysI & cys] \\
\hline Leucine Biosynthesis & leuD & leuc & TeuA & leuB & & & & \\
\hline nitrate reduction III (dissimilatory) & narV & narz & narY & & & & & \\
\hline Nucleosides and Nucleotides Biosynthesis & add & nrdD & deoA & pyri & pyrB & & & \\
\hline pyruvate oxidation pathway & poxB & & & & & & & \\
\hline sulfate activation for sulfonation & cysc & cysN & cysD & & & & & \\
\hline Sulfate Reduction & cysc & cysN & cysD & cysI & cysJ & & & \\
\hline Sulfur Compounds Metabolism & cysC & cysN & cysD & cysI & cysj & & & \\
\hline superpathway of leucine, valine, and isoleucine biosynthesis & ilvC & leuD & leuc & leuA & leuB & & & \\
\hline superpathway of sulfate assimilation and cysteine biosynthesis & cysk & cysc & cysN & CysD & cysI & cysJ & & \\
\hline Trehalose Biosynthesis & ots A & & & & & & & \\
\hline Unusual Fatty Acid Biosynthesis & cfa & & & & & & & \\
\hline
\end{tabular}

Pathways with Lower Expression in K2C2 in AqAl Medium

\begin{tabular}{l} 
2-oxopentenoate degradation \\
3-phenylpropanoate and 3-(3-hydroxyphenyl)propanoate degradation to 2-oxopent-4-enoate \\
\hline Alchenypropronata
\end{tabular} 3-phenylpropionate and 3-(3-hydroxyphenyl)propionate degradation

Amines and Polyamines Biosynthesis

Amino Acids Biosynthesis

Aminoacyl-tRNA Charging

Ammonia Assimilation

Aromatic Compounds Degr

aspartate superpathway

Biotin Biosynthesis

biotin biosynthesis from 8-amino-7-oxononanoate $I$

cinnamate and 3-hydroxycinnamate degradation to 2-oxopent-4-enoate

D-sorbitol degradation II

fucose degradation

Glycine Degradation

glycolate and glyoxylate degradation II

Histidine Biosynthesis

Metabolic Clusters

Other Amino Acid Biosynthesis

superpathway of $\langle i>S</ i>$-adenosyl-L-methionine biosynthesis

superpathway of arginine and polyamine biosynthesis

superpathway of fucose and hamnose degradation

superpathway of glycol metabolism and degradation

Vitamins Biosynthesis

ZraSR Two-Component Signal Transduction System

\begin{tabular}{|c|c|c|c|c|c|c|c|c|c|c|c|}
\hline \multicolumn{12}{|c|}{ Genes Differentially Expressed } \\
\hline mhpF & mhpE & mhpD & & & & & & & & & \\
\hline hcaE & hcaF & hcaD & hcaB & mhpA & mhpB & $\mathrm{mhpC}$ & & & & & \\
\hline mhpF & mhpE & mhpD & hcaE & hcaF & hcaD & hcaB & mhpA & mhpB & mhpC & & \\
\hline mhpF & glpQ & fuco & glcF & glcE & gICD & glcB & & & & & \\
\hline argD & $\operatorname{argC}$ & $\operatorname{argB}$ & $\operatorname{argA}$ & argF & $\operatorname{argI}$ & argh & argG & & & & \\
\hline hisG & hisI & hisH & hisB & tyrA & aspA & metE & metA & $\operatorname{gln} A$ & IysC & argD & $\operatorname{argC}$ \\
\hline $\operatorname{argB}$ & $\operatorname{argA}$ & $\operatorname{argF}$ & $\operatorname{argI}$ & $\operatorname{argH}$ & argG & $\operatorname{asn} A$ & IysA & tdcB & ansB & arof & \\
\hline cysT & $g \ln V$ & $g \ln W$ & leuP & leuv & leuW & leuT & valT & valX & valu & valY & valz \\
\hline alat & thru & thrV & serU & metU & metw & metT & IysW & glyT & & & \\
\hline \multicolumn{12}{|l|}{$\operatorname{gln} A$} \\
\hline & argC & $\operatorname{argB}$ & $\operatorname{argA}$ & argF & argI & argH & argG & & & & \\
\hline mhpA & hcaB & mhpC & mhpB & hcaE & hcaF & hcaD & mhpF & mhpE & mhpD & & \\
\hline metE & metA & lysC & argD & IysA & & & & & & & \\
\hline biof & bioc & bioA & bioB & biod & & & & & & & \\
\hline bioA & bioB & bioD & & & & & & & & & \\
\hline mhpA & $\mathrm{hcaB}$ & mhpC & mhpB & hcaE & hcaF & hcaD & & & & & \\
\hline \multicolumn{12}{|l|}{ srID } \\
\hline fucU & fucA & & & & & & & & & & \\
\hline gcvH & gcvT & gcvP & & & & & & & & & \\
\hline glcF & glcE & glcD & glcB & & & & & & & & \\
\hline glcF & glcE & glcD & glcB & & & & & & & & \\
\hline hisG & hisI & hisH & hisB & & & & & & & & \\
\hline $\begin{array}{c}\text { ansB } \\
\text { valu }\end{array}$ & $\begin{array}{l}\text { asnA } \\
\text { valy }\end{array}$ & $\begin{array}{c}\text { aspA } \\
\text { valz }\end{array}$ & $\begin{array}{l}\text { cyst } \\
\text { alaT }\end{array}$ & $\begin{array}{l}\operatorname{gln} V \\
\text { thrU }\end{array}$ & $\begin{array}{l}\ln W \\
\text { thrv }\end{array}$ & $\begin{array}{l}\text { leuP } \\
\text { serU }\end{array}$ & $\begin{array}{l}\text { leuv } \\
\text { motu }\end{array}$ & $\begin{array}{l}\text { leuW } \\
\text { metw }\end{array}$ & $\begin{array}{l}\text { leut } \\
\text { mett }\end{array}$ & $\begin{array}{l}\text { valT } \\
\text { IyswW }\end{array}$ & $\begin{array}{l}\text { valX } \\
\text { glyT }\end{array}$ \\
\hline IysC & $\frac{\text { valy }}{\text { argD }}$ & $\frac{\text { valz }}{\text { argC }}$ & $\begin{array}{l}\text { alat } \\
\text { argB }\end{array}$ & $\frac{t h r u}{\operatorname{argA}}$ & & & & & & & \\
\hline lysC & metE & metA & & & & & & & & & \\
\hline argD & argC & argB & $\arg A$ & argF & $\operatorname{argI}$ & $\operatorname{argh}$ & argG & & & & \\
\hline fuco & fucU & fucA & & & & & & & & & \\
\hline fuco & glcF & glcE & glcD & glcB & & & & & & & \\
\hline tdcB & tdcD & tdcE & & & & & & & & & \\
\hline gcvH & metF & gcvT & gcvP & biof & bioc & bioA & bioB & biod & & & \\
\hline zraS & & & & & & & & & & & \\
\hline
\end{tabular}




\subsubsection{Higher Differential Expression in K2C2 in AqAl Medium}

Acid resistance stands out as a pathway uniquely overexpressed in $\mathrm{K} 2 \mathrm{C} 2$ in $\mathrm{AqAl}$ medium, and the gad genes involved are two of the highest-fold differentially expressed genes overall. These genes specifically are important to the metabolism of glutamic acid [87]. Since the majority of Nannochloropsis protein content is glutamate [88], the overexpression of these genes in $\mathrm{K} 2 \mathrm{C} 2$ likely helps the strain in both acid neutralization as well as eventual consumption of glutamic acid as a carbon source. These genes are actually downregulated in $\mathrm{K} 2 \mathrm{C} 2$ between Complete MOPS and AqAl media, yet their expression still remains higher than that of K12.

"Unusual" fatty acid synthesis, specifically cyclopropane fatty acid, is also overexpressed through the $c f a$ gene. This essential cell membrane fatty acid is known to be important to acid stress in E. coli [89]. Trehalose production is also overexpressed by K2C2 in AqAl medium via the otsA gene, which is a known response to osmotic stress [90]. The $\operatorname{gad} A / \operatorname{gad} B, c f a$, and otsA genes are all linked in expression to a high-level regulator $r p o S$ which is known to be important to various stress tolerances specifically during stationary phase [91]. However, RNA for this project was harvested during log phase growth and there was no differential expression of $r p o S$ between $\mathrm{K} 2 \mathrm{C} 2$ and parent $\mathrm{K} 12$ in either AqAl or Complete MOPS media, neither were there any mutations detected in the gene itself. Strain K2C2 may have acquired alternate regulatory means of expressing these genes during log growth.

Assimilation of sulfurous compounds via cysteine production is another overexpressed pathway for $\mathrm{K} 2 \mathrm{C} 2$ in AqAl. Stress by furfural, a common byproduct of biomass hydrolysis, has been found to limit sulfur assimilation in E. coli [92], so this may be a means of counteracting a similar stress.

K2C2 also shows a higher expression in branched chain amino acid production genes, both in AqAl and Complete MOPS media. Along with the mutations found in acetolactate synthase subunit genes ilvI, ilvN, and ilvH observed in many different evolved E. coli strains, this further indicates AqAl has some sort of inhibitory effect on branched chain amino acid production. 


\subsubsection{Lower Differential Expression in K2C2 in AqAl Medium}

K2C2 shows a lower expression of cinnamate and other phenolic compound metabolism pathway genes in AqAl medium when compared to parent strain K12. However, both strains show an increase in expression of these pathways between Complete MOPS and AqAl media. The parent strain may be "overshooting" its response to metabolism of these compounds, with $\mathrm{K} 2 \mathrm{C} 2$ having developed a more appropriate level of expression. Alternately, there could be toxic intermediate compounds formed from the associated enzymes after reaction of the intended substrates (or even similarly-structured, unintended substrates), allowing K2C2 to fare better via lower expression.

K2C2 also has a lower expression of ammonia assimilation in AqAl through the $g \ln A$ gene, while showing higher nitrate reduction through nar genes. While not found in strain $\mathrm{K} 2 \mathrm{C} 2$, other strains in evolution Lineage 3 contain an inactivation mutation in the ptsP gene, which also is involved in ammonia assimilation [83]. This seems to confirm that the non-ideal, nitrogen-rich environment in $\mathrm{AqAl}$ is an important issue.

There is a lower level of $\mathrm{K} 2 \mathrm{C} 2$ expression of the biosynthesis pathways for various amino acids, specifically in AqAl. Considering that eight amino acids are expressed at a lower level while the three specific branched chain amino acids are expressed more, this could be a diversion of resources to an amino acid pool threatened by AqAl.

\subsubsection{Expression Changes Between Complete MOPS and AqAI Media}

Both $\mathrm{K} 2 \mathrm{C} 2$ and parent $\mathrm{K} 12$ strains share many up and down regulatory changes between Complete MOPS and AqAl media. These changes do little to show evolutionary changes in the $\mathrm{K} 2 \mathrm{C} 2$ strain, but they do help provide some insight into the stress effects imposed by AqAl media. Both strains up-regulate purine and pyrimidine biosynthesis while down-regulating DNA degradation, showing that AqAl likely stresses DNA synthesis and/or repair. In a similar fashion, Lipid A core biosynthesis is enhanced and fatty acid and lipid degredation is reduced, showing a likely stress on the cell membrane. However, other phospholipid biosynthesis genes are reduced, suggesting a possible reallocation of resources to specific membrane lipids. The waa locus genes responsible for lipid A core synthesis, all upgraded in an AqAl environment, have been shown to be important in outermembrane vesicle formation in E. coli during membrane stress [93]. 
One of the few down-regulations specific to strain $\mathrm{K} 2 \mathrm{C} 2$ is the lower expression of glycolysis pathways when grown on AqAl media. Glucose is the only carbon source in Complete MOPS media and is the preferred carbon source of $E$. coli. There is no detectable glucose in $\mathrm{AqAl}$, so the evolved $\mathrm{K} 2 \mathrm{C} 2$ strain may be more suited to grow immediately on different carbon sources, while the parent strain is still expressing pathways for metabolism of an absent energy source. 
Table 4.3 Pathways differentially expressed between Complete MOPS and 30 vol\% AqAl media growthin in K2C2. Highlighted cells represent pathways differentially expressed in $\mathrm{K} 12$ under the same conditions.

\begin{tabular}{|c|c|}
\hline $\begin{array}{l}\text { Pathways with Higher Expression in K2C2 } \\
\text { between Complete MOPS and AqAI Media }\end{array}$ & $\begin{array}{l}\text { Pathways with Lower Expression in K2C2 } \\
\text { between Complete MOPS and AqAI Media }\end{array}$ \\
\hline 2-oxoglutarate decarboxylation to succinyl-CoA & Acid Resistance \\
\hline 2-oxopentenoate degradation & Alanine Biosynthesis \\
\hline $\begin{array}{l}\text { 3-phenylpropanoate and 3-(3- } \\
\text { hydroxyphenyl)propanoate degradation to 2-oxopent-4- } \\
\text { enoate }\end{array}$ & Alanine Degradation \\
\hline $\begin{array}{l}\text { 3-phenylpropionate and 3-(3-hydroxyphenyl)propionate } \\
\text { degradation }\end{array}$ & Alcohols Degradation \\
\hline ADP-sugar Biosynthesis & Antibiotic Resistance \\
\hline Aerobic Respiration & Arginine Degradation \\
\hline Amino Acids Biosynthesis & arginine degradation II (AST pathway) \\
\hline anhydromuropeptides recycling & AtoSC Two-Component Signal Transduction System \\
\hline Arginine Biosynthesis & Degradation/Utilization/Assimilation \\
\hline Aromatic Compounds Degradation & Detoxification \\
\hline Arsenate Detoxification & Fatty Acid and Lipids Degradation \\
\hline aspartate superpathway & Fermentation \\
\hline Biosynthesis & Folate Biosynthesis \\
\hline biotin-carboxyl carrier protein assembly & Generation of Precursor Metabolites and Energy \\
\hline C1 Compounds Utilization and Assimilation & glycerol and glycerophosphodiester degradation \\
\hline $\begin{array}{l}\text { cinnamate and 3-hydroxycinnamate degradation to 2- } \\
\text { oxopent-4-enoate }\end{array}$ & glycolysis I (from glucose-6P) \\
\hline dTDP-sugar Biosynthesis & glycolysis II (from fructose-6P) \\
\hline enterobacterial common antigen biosynthesis & $\begin{array}{l}\text { KdpDE Two-Component Signal Transduction System, } \\
\text { potassium-dependent }\end{array}$ \\
\hline formate to dimethyl sulfoxide electron transfer & L-arabinose Degradation \\
\hline Generation of Precursor Metabolites and Energy & Lactaldehyde Degradation \\
\hline glutathione redox reactions II & NAD Metabolism \\
\hline glycerol degradation $\mathbf{V}$ & nitrate reduction III (dissimilatory) \\
\hline Glycine Degradation & Other Biosynthesis \\
\hline Inorganic Nutrients Metabolism & $\begin{array}{l}\text { PhoQP Two-Component Signal Transduction System, } \\
\text { magnesium-dependent }\end{array}$ \\
\hline Leucine Biosynthesis & $\begin{array}{l}\text { PhoRB Two-Component Signal Transduction System, } \\
\text { phosphate-dependent }\end{array}$ \\
\hline Lipid A-core biosynthesis & Phospholipid Biosynthesis \\
\hline lipoate biosynthesis and incorporation II & polymyxin resistance \\
\hline Lipopolysaccharide Biosynthesis & pyrimidine ribonucleosides degradation \\
\hline Metabolic Clusters & respiration (anaerobic) \\
\hline methylglyoxal degradation I & RstBA Two-Component Signal Transduction System \\
\hline NADH to cytochrome bd oxidase electron transfer & salvage pathways of pyrimidine deoxyribonucleotides \\
\hline NADH to cytochrome bo oxidase electron transfer & Sugar Acids Degradation \\
\hline NADH to dimethyl sulfoxide electron transfer & $\begin{array}{l}\text { superpathway of D-glucarate and D-galactarate } \\
\text { degradation }\end{array}$ \\
\hline NADH to fumarate electron transfer & $\begin{array}{l}\text { superpathway of hexuronide and hexuronate } \\
\text { degradation }\end{array}$ \\
\hline NADH to trimethylamine $\mathbf{N}$-oxide electron transfer & $\begin{array}{l}\text { superpathway of purine deoxyribonucleosides } \\
\text { degradation }\end{array}$ \\
\hline nitrate reduction VIII (dissimilatory) & $\begin{array}{l}\text { superpathway of pyrimidine deoxyribonucleosides } \\
\text { degradation }\end{array}$ \\
\hline Nitrogen Compounds Metabolism & \\
\hline Nitrogen Regulation Two-Component System & \\
\hline Nucleosides and Nucleotides Biosynthesis & \\
\hline O-Antigen Biosynthesis & \\
\hline pyruvate decarboxylation to acetyl CoA & \\
\hline superpathway of chorismate metabolism & \\
\hline superpathway of fatty acid biosynthesis I & \\
\hline $\begin{array}{l}\text { superpathway of histidine, purine, and pyrimidine } \\
\text { biosynthesis }\end{array}$ & \\
\hline superpathway of lipopolysaccharide biosynthesis & \\
\hline TCA cycle I (prokaryotic) & \\
\hline Vitamins Biosynthesis & \\
\hline
\end{tabular}




\subsection{Discussion and Conclusions}

The E. coli and P. putida strains produced in the adaptive evolution study were shown to grow faster and to a higher yield than their parent strains when grown in media using AqAl as the sole C/N/P source. Illumina full-genome sequencing analysis yielded a list of single nucleotide polymorphism mutations likely responsible for the improvements. The mutations were not limited to one type of gene or class of genes, but varied over many functions, including substrate metabolism, transport, regulatory processes, and cell synthesis. This indicates that there is likely an assortment of selective properties in the complex aqueous phase product that can be addressed and improved upon via evolution.

These improved strains and known mutations could provide a functional benefit to any group seeking to use aqueous phase from hydrothermal liquefaction of biomass as a substrate and nutrient source for a microbial growth operation.

Among these improved strains are both E. coli and P. putida that cross-contaminated the other's cultures during the serial passaging process. Yet neither organism overpowered the other and both were found coexisting in terminal cultures, suggesting each found a niche that was mutually beneficial or at least non-competitive.

We also implemented a select group of six E. coli mutations into a laboratory strain's chromosome via homologous recombination in order to study their individual effects. All mutations provided some sort of benefit to E. coli when grown in AqAl media. Two mutations, glpKB and ilvN showed a clear improvement and detriment (respectively) when a control minimal medium with defined $\mathrm{C} / \mathrm{N} / \mathrm{P}$ sources was used, while the others had minimal effects, showing that most of these mutations had mechanisms of action specific to factors introduced by AqAl.

Mutations in the glycerol-metabolism glpK gene greatly improved growth rate of $E$. coli when glycerol was the only available carbon source. AqAl has been shown previously to contain a small amount of glycerol in addition to a large amount of unknown organic carbon compounds [77], so it is not surprising to see an evolved strain that consumes it more readily. Also, the $g l p K$ mutations were only found in $E$. coli that had contaminated $P$. 
putida cultures, and evolved $P$. putida strains tended to grow faster and to a higher density than E. coli, but with a longer lag phase. So glpK mutant E. coli may be able to compete with the otherwise dominant $P$. putida by quickly metabolizing glycerol first.

The $p t s P$ mutation was tested in media of various carbon to nitrogen ratios, since it is known to regulate the import of sources of each. The mutation provided a slight advantage over a wide range of $\mathrm{C}: \mathrm{N}$ ratios, but not of the magnitude observed for AqAl media. Further work with more extreme ranges of C:N ratios and the inclusion of complex organic nitrogen sources, instead of just ammonia, may be necessary to show a direct effect.

Mutations in the ilv acetolactate synthase genes were tested with compounds suspected to be present in AqAl that are known imidazolinone-class acetolactate synthase inhibitors in plants. IlvI and ilvH showed a slight benefit towards imazaquin growth inhibition. But more apparent results are needed before a conclusion can be made. One option would be to attempt to measure the activity of mutants' acetolactate synthase enzyme in the presence of AqAl in comparison to the wild type strain. This could confirm that the enzyme is indeed resistant to inhibition from some compound (or mix of compounds) in AqAl, even if the compound identities remain unknown. 


\subsection{Materials and Methods}

We separated aqueous phase (AqAl) from the hydrothermal liquefaction of algae biomass and used it as the sole Carbon, Nitrogen, and Phosphorus source in bacterial growth media. We evolved two bacteria species, Escherichia coli and Pseudomonas putida in AqAl media via serial passaging and examined growth phenotypes of evolved cultures and clonal isolates thereof. Isolates showing the most difference from their parent strain were subjected to full genetic sequencing to determine single nucleotide polymorphisms (SNPs) that developed over the course of evolution. A set of these SNPs in E. coli were introduced to a laboratory strain individually and tested against the base strain to determine individual contributions to AqAl tolerance.

We also ran a full RNA transcriptome sequencing analysis on the top-performing $E$. coli isolate and its parent strain after growth in both standard minimal media and AqAl minimal media in order to determine their difference in genetic regulation under these conditions.

\subsubsection{Hydrothermal Liquefaction}

We purchased Nannochloropsis oculata microalgae from Reed Mariculture Inc. as our source of biomass, since this strain is often used in hydrothermal liquefaction studies due to its high oil yields. Deionized water was combined with the biomass to make a 20 wt\% solids slurry, and $150 \mathrm{ml}$ of the mixture was used in each hydrothermal liquefaction (HTL) reaction. We used a Parr 4570 Pressure Reactor with a total internal volume of 283 $\mathrm{ml}$, heated by a LC Miller PR-15AB induction coil heater. The reactor was heated to a temperature of $350{ }^{\circ} \mathrm{C}$ (taking an average of $8.7 \mathrm{~min}$ to reach target temperature) and held within two degrees of that target for one hour while being agitated by an impeller at 800 rpm. Reactor pressure stabilized at roughly 100 bar during the reaction. Afterward, the reactor was cooled, the gas product was vented, and the contents of the reactor were mixed with $100 \mathrm{ml}$ of dichloromethane (Optima grade, $>95 \%$ purity) and transferred to a separatory funnel. The solids and dichloromethane-soluble product fraction (containing the "bio-crude" oil) were decanted and the water-soluble fraction was isolated and ran through a Corning $0.2 \mu \mathrm{m}$ cellulose acetate filter in order to remove any residual solids or 
oil. We considered the resulting product "raw AqAl" and combined AqAl from multiple batches in order to make a single amount large enough to run the entire evolution serial passaging study with. Before use in any media, AqAl was neutralized to a $\mathrm{pH}$ of seven with hydrochloric acid.

\subsubsection{Media formulation}

The base medium for bacterial growth was based on a standard organic-buffered 3(N-morpholino)pro-panesulfonic acid (MOPS) medium for enterobacteria [94]. The medium contained $0.04 \mathrm{M}$ MOPS and $0.004 \mathrm{M}$ tricine as buffers, adjusted to a $\mathrm{pH}$ of 7.4 with $\mathrm{KOH}$. It also contained $2.91 \mathrm{nM}\left(\mathrm{NH}_{4}\right)_{6} \mathrm{Mo}_{7} \mathrm{O}_{2}, 401.1 \mathrm{nM} \mathrm{H}_{3} \mathrm{BO}_{3}, 30.3 \mathrm{nM} \mathrm{CoCl}_{2}\left(\mathrm{H}_{2} \mathrm{O}\right)_{6}, 9.61 \mathrm{nM}$ $\mathrm{CuSO}_{4}\left(\mathrm{H}_{2} \mathrm{O}\right)_{5}, 51.4 \mathrm{nM} \mathrm{MnCl} 2,6.1 \mathrm{nM} \mathrm{ZnSO}_{4} \mathrm{H}_{2} \mathrm{O}, 0.01 \mathrm{mM} \mathrm{FeSO}_{4}\left(\mathrm{H}_{2} \mathrm{O}\right)_{7}, 1 \mathrm{mM} \mathrm{Mg}_{2} \mathrm{SO}_{4}$, and $0.1 \mathrm{mM} \mathrm{CaCl}$, sterilized with a Corning $0.2 \mu \mathrm{m}$ vacuum filtration system. This formulation itself was referred to as "stripped MOPS" and all media used in this study shared at least these ingredients. We added $2 \mathrm{~g} / \mathrm{L}$ glucose, $1 \mathrm{~g} / \mathrm{L} \mathrm{NH} \mathrm{NH}_{4} \mathrm{Cl} 0.23 \mathrm{~g} / \mathrm{L} \mathrm{K} \mathrm{KPO}_{4}$ dibasic (for $\mathrm{C} / \mathrm{N} / \mathrm{P}$ sources) and $0.5 \mathrm{~g} / \mathrm{L} \mathrm{NaCl}$ to Stripped MOPS medium to make Complete MOPS medium, the reference minimal medium used for the study. We also combined Stripped MOPS with 10-30 vol\% AqAl to make AqAl media, used as media for serial passaging during evolution as well as various growth phenotype tests.

For later growth tests featuring E. coli strain ECHW24 and mutants derived from it we added biotin to the media at $0.25 \mathrm{mg} / \mathrm{L}$ due to the strain missing its biotin locus. For a test with glpk mutant strains we used Complete MOPS media with various concentrations of glycerol substituted for glucose. With the pstP mutant strain we used Complete MOPS media with various ratios of carbon and nitrogen by altering ammonia levels. We also grew ilvI, ilvN, and ilvH mutants with Complete MOPS spikes with varying levels of known acetolactate synthase enzyme inhibitor compounds imazaquin, imazapyr, and imazamethabenz.

\subsubsection{Adaptive Evolution}

In a previous study, bacteria strains Escherichia coli K12 MG1655 and Pseudomonas putida KT2440 showed the ability to grow using AqAl as the sole C/N/P source, although substrate usage was quite inefficient and there was a considerable decline in growth in media above $20 \mathrm{vol} \% \mathrm{AqAl}$ [77]. We chose to use E. coli since it was thoroughly researched, 
much is known about its genome and individual gene function, and there are available tools and methods for its genetic manipulation (important for eventually interpreting the results of full-genome sequencing and introduction of individual mutations). P. putida is known for its diverse metabolic network and may be more suited for growth on the complex substrate mix in AqAl.

For this project we used E. coli and P. putida as "parent strains" for an adaptive evolution study, with AqAl providing the selective pressure on continually passaged cultures of each organism. Cryostock samples of each organism were grown in $10 \mathrm{vol} \%$ AqAl media overnight at $30^{\circ} \mathrm{C}$ in a New Brunswick E24 incubator agitated at $250 \mathrm{rpm}$, then $40 \mu \mathrm{L}$ of each culture were added to three $15 \mathrm{~mL}$ sterile plastic tubes containing $2 \mathrm{~mL}$ of fresh media. These six cultures were treated as biological replicates of the same evolution study (Lineages) and will be referred to as Lineages K1, K2, and K3 (for E. coli K12) and P1, P2, P3 (for P. putida).

Cultures were grown in the same incubation conditions $\left(30^{\circ} \mathrm{C}, 250 \mathrm{rpm}\right)$ and every two to three days (Monday, Wednesday, and Friday of every week) a $40 \mu \mathrm{L}$ sample of each culture was transferred to $2 \mathrm{~mL}$ of fresh media, each incubation/transfer period constituting one "Passage". Each Passage contained five to six doublings (or "Generations") of cells during exponential growth over the course of roughly one day, followed by a stationary phase.

Cultures were passaged in this manner 60 times. Cultures from every one to five Passages were cryogenically preserved by combining equal parts sterile $50 \mathrm{wt} \%$ glycerol solution and cell culture and storing at $-80{ }^{\circ} \mathrm{C}$. At Passage 32 we increased the AqAl concentration in the media to $20 \mathrm{vol} \%$, and then to $30 \mathrm{vol} \%$ at Passage 56 . We concluded the experiment at Passage 60 and made a final cryostock of each Lineage culture. We also spread dilutions of each culture on $10 \mathrm{vol} \% \mathrm{AqAl}$ media agar plates, grew them overnight at $30^{\circ} \mathrm{C}$, picked ten isogenic colonies for each Lineage, and made individual cultures and cryostocks of them. These strains were named with the convention "Organism-LineageClone-\#", so strain K2C2, for example, is derived from the second colony isolated from $E$. coli K12 Lineage Two. These strains were deemed "Sequencing Candidates", and were 
further screened for phenotypic differences from their parent strains to determine if mutations improving AqAl tolerance had likely taken place.

\subsubsection{Phenotype Testing of Sequencing Candidates}

We performed multiple types of growth tests on individual Sequencing Candidates derived from the evolution project. The properties measured were growth rate, maximum cell density (inferred from optical density), and organic carbon consumption. Cultures were grown in either $200 \mu \mathrm{L}$ volumes in 96-well microplates so that optical density measurements could be taken automatically every ten minutes, or $2 \mathrm{~mL}$ volumes in capped $15 \mathrm{~mL}$ Falcon-brand plastic tubes so that a larger amount of culture supernatant could be harvested.

\subsubsection{Inoculum Generation}

For all growth phenotype tests, $2 \mathrm{~mL}$ cultures of the strains involved (from $-80{ }^{\circ} \mathrm{C}$ cryostock samples) were grown overnight $\left(30^{\circ} \mathrm{C}, 250 \mathrm{rpm}\right)$ in $20 \mathrm{vol} \% \mathrm{AqAl}$ media. The cells were then washed once with $1 \mathrm{ml}$ sterile deionized water through centrifugation and resuspension with an Eppendorf 5415C benchtop centrifuge at $16000 \mathrm{rcf}$ for one minute, centrifuged again, then resuspended in an amount of DI water such that the OD650 was equal to 2.0. Then a specific amount of each inoculum suspension was used in each test such that all cultures compared would have an approximately identical amount of starting viable cells.

\subsubsection{Growth Curve Generation}

We used a Molecular Devices Versamax or Spectramax M5 microplate reader to agitate and incubate $200 \mu \mathrm{L}$ cell cultures in a 96-well Corning microplate. The devices kept cultures at a temperature of $30^{\circ} \mathrm{C}$ and took optical density (OD) readings at $650 \mathrm{~nm}$ every ten minutes such that kinetic growth data could be generated over several days. We used $650 \mathrm{~nm}$, instead of the more standard $600 \mathrm{~nm}$ for measuring bacterial culture turbidity, since AqAl has a high native $600 \mathrm{~nm}$ absorbance.

These microplate tests provided a maximum OD value achieved for each culture, as

well as growth rate (both measured as the average of three replicates). AqAl contains a mixture of bioavailable substrates such that cultures often exhibited multiple diauxic growth phases. We chose the most prominent exponential growth phase (the one taking 
place over the longest period of time) and determined the Monod kinetics model maximum specific growth rate $\left(\mu_{\max }, \mathrm{hr}^{-1}\right)$ by fitting a line to a log-plot of OD vs. time [95].

\subsubsection{Organic Carbon Consumption Tube Tests}

We grew $2 \mathrm{~mL}$ cell cultures of the sequencing candidate strains as well as their parent strains for 48 hours $\left(30^{\circ} \mathrm{C}, 250 \mathrm{rpm}\right)$ in $20 \mathrm{vol} \%$ AqAl medium, measured the final OD650, sterile-filtered the culture, and collected the supernatant. The cell-free "spent media" was examined with a Shimadzu TOC-VCSH Organic Carbon Analyzer. Organic carbon consumption was inferred from the difference between pre and post-culture media.

\subsubsection{Selection of Sequencing Strains of Interest}

We narrowed down the ten "sequencing candidate" strains from each final evolution lineage to three sequencing "strains of interest" based on their phenotype difference from the parent strain. The four phenotypes considered were microplate study max OD650, microplate study $\mu_{\max }$, tube test max OD650, and tube test organic carbon consumption. Unitless numbers indicating overall improvement in phenotype over the parent strain as well as overall difference from the parent strain were generated via the following formulas: 


$$
\text { improvement }=\sum_{i=1}^{4} \frac{s_{i}-p_{i}}{p_{i}} \quad \text { difference }=\sum_{i=1}^{4}\left|\frac{s_{i}-p_{i}}{p_{i}}\right|
$$

Where:

$\mathrm{s}_{\mathrm{i}}=$ Strain of interest phenotype

$\mathrm{p}_{\mathrm{i}}=$ Parent phenotype

For all four phenotypes, a greater value than that of the parent was considered an improvement, so the sum of their relative differences (with negative values reducing the sum) was considered the overall improvement. The overall difference of the strains was defined as the sum of the absolute values of the relative differences.

From each set of ten isolated strains from each Lineage, we picked the two with the highest overall difference values as well as the one with the lowest overall improvement for investigation via full genome sequencing. The goal of this screening was to choose strains with the most varied sets of mutations.

\subsubsection{Species Verification}

A polymerase chain reaction (PCR) was performed on each of the Strains of Interest that amplified the majority ( $\sim 1.5 \mathrm{~kb})$ of the 16S RNA gene (sequence in Table 4.5$)$, a gene commonly used to identify bacteria due to it having certain regions tightly conserved among most bacteria species (allowing one primer set to be suitable for all bacteria) as well as certain regions that are species dependent. The primers were synthesized by Integrated DNA Technologies. The fragments were amplified using 5 PRIME Master Mix-brand premade PCR polymerase/dNTP mix using the manufacturer's recommended thermocycler protocol and annealing temperatures no higher than the lowest supplier-calculated melting temperature of each primer pair. One $\mu \mathrm{L}$ of overnight cell culture was used as the DNA template for the reactions. Correct fragment size and lack of side products was confirmed via gel electrophoresis using 1\% agarose in tris-borate-EDTA gels and ethidium bromide as a DNA-binding fluorescent stain. These fragments were purified with Qiagen-brand 
Qiaquick kits and sent for Sanger genetic sequencing at the University of Michigan. Chromatograms of the data were manually inspected. Sequencing results were analyzed with the National Center for Biotechnology Information Basic Local Alignment Search Tool (BLAST) and showed unique 100\% genetic homology with either E. coli MG1655 or P. putida KT2440.

However, two strains from the E. coli Lineage 1 group were shown to be E. coli and one strain from each $P$. putida Lineage was E. coli. This implied that no foreign organisms contaminated the evolution experiment, but there had been a cross-contamination between cultures. We confirmed this by growing final lineage cryostocks on agar plates coated with Fisher-brand Chromomax (an IPTG/X-Gal solution), which causes blue colony color for organisms with a lacZ operon, which E. coli contains but P. putida lacks. The Lineages with suspected contamination all contained colonies of mixed colors, showing definite contamination. It is unknown when this contamination happened, but the mix of species in each affected terminal evolution Lineage indicates that no contaminant organism completely took over the population.

\subsubsection{Full Genome Sequencing}

The strains of interest, along with their parent strains, were grown overnight in 30 vol\% AqAl medium and genomic DNA was extracted with Qiagen-brand DNEasy kits. Samples were sent to the University of Michigan Sequencing Core.

Sequencing Core personnel fragmented the DNA to 400bp using standard Covaris sonication. Fragmented DNA was then prepared as a standard Illumina library using IntegenX reagents on an Apollo instrument. (fragmented, end repaired, A tailed, adapter ligated and PCR amplified) and pooled ( $\sim 20$ libraries per pool). Final libraries were checked for quality and quantity by Agilent TapeStation and qPCR using Kapa's library quantification kit for Illumina Sequencing platforms (catalog \# KK4835). They were clustered and sequenced 20 samples per lane in 50 cycle single end mode on a HiSeq 2000 Illumina Genome Analyzer in High Output mode using Version 3 reagents according to manufacturer's protocols. 
This device sequenced roughly $50 \mathrm{bp}$ from the end of each fragment, yielding data that provided roughly 60-100X genome coverage (based on total genome size, total library size, and assumed equal representation throughout the genome). In other words, any given base pair in the genome should be represented within 60 to 100 of the 50bp data fragments.

We used the custom Perl script from Minty et al. to align the data fragments for each strain to form a consensus sequence [42]. This script utilized Novoalign v2.04.02 to generate a list of single nucleotide polyorphisms (SNPs) in each Strain of Interest when compared to its parent strains' (Escherichia coli K12 MG1655 or Pseudomonas putida KT2440) known genome sequences, retrieved from the NCBI database. Identities and functions of the genes affected, along with amino acid residue changes due to the mutations, were determined by referencing the Kyoto Encyclopedia of Genes and Genomes (KEGG) database. Several SNPs were identified in all strains of a given species, including the parent strain. We removed these from our results, assuming they were due to either mutations our lab strain contained before the evolution project began, or possibly errors in the NCBI sequence itself. We also disregarded any mutations appearing in fewer than $90 \%$ of the aligned sequence fragments containing the nucleotide in question.

At this point we ceased genetic sequence processing for the $P$. putida strains. For the E. coli strains, we confirmed each mutation via Sanger sequencing and introduced select mutations into a lab strain of $E$. coli for individual study.

\subsubsection{Sanger Sequencing Confirmation of SNPS}

DNA fragments in all E. coli Strains of Interest containing the suspected SNPs were amplified using the PCR materials and protocol mentioned in Section 4.5.9 (sequence in Table 4.5). Fragment size/specificity, DNA purification, and Sanger Sequencing were carried out in the same fashion as well. All SNPs suspected were confirmed. The parent $E$. coli strain was also sequenced at all suspected SNP sites to confirm the mutations were not present at the beginning of the evolution experiment. 


\subsubsection{Homologous Recombination}

We implemented individual SNPs of interest onto the chromosome of $E$. coli EcHW24. This strain contains " $\lambda$-Red" genes as well as a deactivated mutS gene which render it very susceptible to incorporation of partially homologous ssDNA oligomers under certain conditions. We used a method first defined by Wang et al then modified by Minty et al for this process [42], [96].

Six SNP mutations were chosen for investigation, with locations in the ilvI, ilvN, ilvH, $p t s P$, and $g l p K$ (two mutations) genes. DNA Oligomers $90 \mathrm{bp}$ in length were designed that were homologous to the lagging-replication fork strand in these genes but with the appropriate single nucleotide exchanged in the middle. The fragments all had a $\Delta \mathrm{G}$ greater than $-12.5 \mathrm{kcal} / \mathrm{mol}$ for secondary structures (calculated with mfold software: http://mfold.rna.albany.edu/?q=mfold). The 90-mer oligos were produced by Integrated DNA Technologies and contained four 5' phosphorothioated base pairs to enhance stability (sequence in Table 4.5).

To prepare cells for recombination, two mL EcHW24 cultures were grown overnight at $30{ }^{\circ} \mathrm{C}$ in $\mathrm{LB}$ broth. One $\mathrm{mL}$ of culture was added into $10 \mathrm{~mL}$ of fresh $\mathrm{LB}$ broth and incubated again until its OD600 was 0.6-0.7 (done to catch the cells in mid-log growth phase). Cultures were then heated in a $42{ }^{\circ} \mathrm{C}$ water bath for 15 minutes in order to activate the $\lambda$-Red genes then immediately chilled in an ice bath for ten minutes. From this point until eventual electroporation, cells were kept in either an ice bath or a cold room with a temperature of $4{ }^{\circ} \mathrm{C}$ to slow cellular processes and prevent any "distress" functions from being activated.

Cultures were centrifuged in an Eppendorf 5415C benchtop centrifuge at $16000 \mathrm{rcf}$ for one minute. The supernatant was removed, and cells were resuspended in $1 \mathrm{~mL}$ of UltraPure-brand DNase/RNase-free water. This was repeated twice more in order to prepare recombination-competent cells, with final resuspension in only $50 \mu \mathrm{L}$ of water. The competent cells were then transferred to an electroporation cuvette and combined with a 90-mer (described previously) for a final DNA concentration of $5 \mu \mathrm{M}$ and exposed to a 1.8 $\mathrm{kV}$ shock in an Eppendorf Electroporator 2510. The cell/DNA suspension was then mixed 
with $5 \mathrm{~mL}$ of LB medium and allowed to recover at $30^{\circ} \mathrm{C}$ for 30 minutes to three hours. The recovered cultures were then diluted $1: 10^{4}$ to $1: 10^{5}$ and $100 \mu \mathrm{L}$ of each were spread on LB agar plates and incubated overnight at $30^{\circ} \mathrm{C}$.

This method for recombination is reported to be efficient enough (1\%-40\% success rate) to avoid selection factors and instead directly screen individual colonies via Sanger sequencing. However, since efficiencies could be as low as 1\%, allele-specific PCR primers (intended to only amplify DNA from successful SNP mutants) were designed so a large amount of colonies could be screened first with allele-specific PCR then only ones yielding a product could be sent for confirmation via Sanger sequencing.

Allele-specific primer pairs were designed for each of the six SNP mutations investigated containing one primer with a single nucleotide on its 3' end corresponding to the SNP nucleotide (sequence in Table 4.5). This promotes fragment elongation of only DNA templates containing the right SNP. The design was carried out using the WASP allelespecific primer design tool (http://bioinfo.biotec.or.th/WASP). The primers were synthesized by Integrated DNA Technologies. The PCR reagents and cycling conditions we used in allele-specific experiments were identical to those described in Section 4.5.9. However, the manufacturer-calculated melting temperatures of primers are not very useful when deciding annealing temperatures for allele-specificity, so gradient tests over large ranges of annealing temperatures with appropriate negative and positive controls (EcHW24 and confirmed mutant Strains of Interest, respectively) were carried out to determine the most specific annealing temperatures. For some primer pairs, no such annealing temperature was found, so primers were redesigned (also with WASP) containing the SNP-specific 3' nucleotide plus an additional flanking nucleotide with a mismatch. These primers were confirmed to function as intended.

Individual colonies from the electroporation experiments were picked and screened via allele-specific PCR in groups of 15 to 30. The colonies were suspended in $100 \mu \mathrm{L}$ of sterile water and $1 \mu \mathrm{L}$ of suspension was used as the DNA template for the PCR. Positive and negative control strains were analyzed on each PCR run to ensure the allele-specific primers were working with the intended specificity. If a colony showed a product from the 
PCR, it was subcloned (the cell suspension was plated and incubated overnight again) and colonies from the new plate were picked and screened again. We did this since previous experience with this recombination technique showed that the EcHW24 strain is prone to making polyploid cells after the electroporation process and still often contains multiple chromosome copies even after several hours of recovery and cell division. Thus agar plate colonies from dilutions of these cultures may not be perfectly clonal and contain monoploid cells both with and without the SNP. The second round of sub-cloning usually guarantees pure genotypes in each colony.

Subclones that produced allele-specific PCR products were then confirmed via the process described in the Section 4.5.11. At least one successful mutant was found for each SNP containing only the SNP nucleotide in its amplified DNA fragment (i.e. no interfering wild-type nucleotide peak was present in the chromatogram data, meaning there was no mixed genotype due to polyploidy complications). The cell suspensions of these mutants were added to $2 \mathrm{~mL}$ of LB media, grown overnight at $30^{\circ} \mathrm{C}$, combined in equal parts with sterile $50 \mathrm{wt} \%$ glycerol solution, and stored at $-80^{\circ} \mathrm{C}$ as "Individual Mutants" cryostocks for further experimentation.

\subsubsection{Growth Phenotype Testing of Strains of Interest and Individual Mutants}

We performed several microplate reader growth experiment studies in the same fashion as described in Section 4.5.6 in order to compare evolved Strains of Interest of both species to their parent strains (K12 MG1655 for E. coli and KT2440 for P. putida) and also compare Individual Mutant E. coli to their EcHW24 parent strain. Cultures in 30 vol\% AqAl media were grown in either duplicate or triplicate with parent strains always located on the same plate used as cultures of their evolved/mutated derivatives. We also grew each strain in standard Complete MOPS medium.

We used the data generated to determine maximum OD change in the same way we did for the Sequencing Candidate strains. However, we measured growth rate in a different fashion. Instead of fitting the longest of the several diauxic growth phases to a Monod $\mu_{\max }$ value (thus ignoring other growth periods) we used a simpler overall growth rate (OGR) to capture an average growth metric. We defined OGR as the difference between maximum 
and initial OD divided by the time between the start of the first exponential growth phase and beginning of stationary phase.

\subsubsection{GlpK Mutant Tests}

The two glpK Individual Mutants (referred to as strains $g l p k A$ and $g l p k B$ ) and strain EcHW24 were grown in a microplate test using Complete MOPS media with varying levels of glucose and glycerol as the carbon source. The media used contained 2.0/0, 1.7/0.3, $0.3 / 1.7$, and $0 / 2.0$ glucose/glycerol concentrations (in $\mathrm{g} / \mathrm{L}$ ) along with all the other components of standard Complete MOPS media. Cultures were grown in triplicate on the same microplate and max OD change and OGR were calculated.

\subsubsection{PtsP Mutant Tests}

The ptsP Individual Mutant and EcHW24 strains were both grown in a microplate study using Complete MOPS media with a set $2.0 \mathrm{~g} / \mathrm{L}$ of glucose and varying levels of ammonia to make media with $\mathrm{C}: \mathrm{N}$ ratios (by weight) of $3,0.8,0.4$, and 0.2 . Cultures were grown in triplicate on the same microplate and max OD change and OGR were calculated.

\subsubsection{Ilv-Family Mutant Tests}

The ilvI, ilvN, and ilvH Individual Mutant and EcHW24 strains were grown in a microplate study using Complete MOPS spiked with varying levels of acetolactate synthase inhibitor compounds suspected to be present in AqAl. The compounds chosen were imazaquin, imazapyr, and imazamethabenz, which we purchased in pure crystalline form from Fisher Scientific. After some preliminary testing to determine toxicity ranges, we chose levels of $100 \mathrm{ppm}$ and $500 \mathrm{ppm}$ (for imazaquin and imazamethabenz) and $500 \mathrm{ppm}$ and 1000 ppm (for imazapyr) as ideal concentrations to investigate further.

A $50 \mathrm{~g} / \mathrm{L}$ imazapyr stock (in sterile DI water) was used to spike Complete MOPS medium to make $500 \mathrm{ppm}$ and $1000 \mathrm{ppm}$ media. Imazaquin and imazamethabenz were difficult to dissolve in water, so were instead dissolved as $50 \mathrm{~g} / \mathrm{L}$ stocks in Dimethyl Sulfoxide (DMSO). These stocks were then used to make $100 \mathrm{ppm}$ and $500 \mathrm{ppm}$ level media. And to standardize any affect the DMSO might have, pure DMSO was added to control Complete MOPS media as well as $100 \mathrm{ppm}$ media to make them contain the same concentration of DMSO (1 vol\%) as the 500 ppm media. Cultures were grown in triplicate and max OD change and OGR were calculated. 


\subsubsection{RNA Transcriptome Sequencing}

The highest-performing Strain of Interest (the one with the highest Max OD and OGR from plate test results) was chosen to be further studied for transcriptional variation in comparison to its parent strain, E. coli K12 MG1655. This strain was designated E. coli "K2C2" due to being derived from the second colony chosen (C2) from the K12 evolution Lineage \#2 (K2).

Both parent and $\mathrm{K} 2 \mathrm{C} 2$ cryostocks were grown overnight at $30{ }^{\circ} \mathrm{C}$ in both Complete MOPS and $30 \mathrm{vol} \%$ AqAl media. The OD650 of each culture was measured and the cultures were spun down in an Eppendorf 5415C benchtop centrifuge at $16000 \mathrm{rcf}$ for one minute. They were then resuspended in $1 \mathrm{ml}$ of sterile DI water, centrifuged again, and resuspended in a volume of water such that their OD650 was approximately 2.0. We then added $20 \mu \mathrm{L}$ of the suspensions to four replicates each of Complete Mops and 30\% AqAl media, for 16 new cultures in total (four parent and K2C2 cultures in Complete MOPS inoculated with cells that had been grown overnight in Complete MOPS, and four parent and K2C2 cultures in 30 vol\% AqAl media inoculated with cells grown overnight in 30 vol\% AqAl media).

These new cultures were incubated at $30^{\circ} \mathrm{C}$ for approximately 12 hours and harvested with cultures having a measured OD650 of 0.20, 0.19, 0.21, and 0.27 for parent-cMOPS, $\mathrm{K} 2 \mathrm{C} 2-\mathrm{cMOPS}$, parent-AqAl, and $\mathrm{K} 2 \mathrm{C} 2-\mathrm{AqAl}$, respectively. This was considered mid-log growth phase, since previous studies that grew identically prepared cultures to stationary phase produced cultures with OD650 measurements of $0.43,0.53,0.3$, and 0.44 . The cultures were each processed with Qiagen-brand RNeasy Mini RNA extraction kits. The optional RNeasy Protect reagent was used to stabilize the RNA after cell lysis, as well as DNase on-column reagent. We followed the manufacturer's recommended protocol for bacterial total RNA extraction. We made suspensions containing $2 \mu \mathrm{g}$ total RNA in $50 \mu \mathrm{L}$ Ultrapure water, froze them at $-80{ }^{\circ} \mathrm{C}$, and sent them to the University of Michigan Sequencing Core.

At the Sequencing Core, RNA samples were assessed for quality using the Agilent TapeStation. Samples with RINs (RNA Integrity Numbers) of 8 or greater were ribosomal RNA depleted using Invitrogen's Ribo Minus kit. The rRNA-depleted samples were then 
prepped using the Illumina TruSeq mRNA Sample Prep v2 kit. The entire fraction of 0.1-3ug of rRNA-depleted total RNA was fragmented and copied into first strand cDNA using reverse transcriptase and random primers. The 3 prime ends of the cDNA were then adenylated and adapters were ligated. One of the adapters that was ligated had a 6 nucleotide barcode that was unique for each sample which allowed them to sequence more than one sample in each lane of a HiSeq flow cell. The products were purified and enriched by PCR to create the final cDNA library. Final libraries were checked for quality and quantity by Agilent TapeStation and qPCR using Kapa's library quantification kit for Illumina sequencing platforms. They were clustered and sequenced 16 samples per lane in a 50 cycle single end read mode on a HiSeq 2000 Illumina Genome Analyzer in High Output mode using Version 3 reagents according to manufacturer's protocols. The analysis produced a combined $\sim 139$ million 52 -bp reads for a total of $\sim 7.25$ billion bp of data. These data generated from this project were sent to the University of Michigan Bioinformatics Core.

The Bioinformatics Core aligned the single-read data for each sample to a reference E. coli genome and quantified each gene's level of expression. Then the four replicates of the four different cell/media combinations (parent in both cMOPS and AqAl media, and $\mathrm{K} 2 \mathrm{C} 2$ in both cMOPS and AqAl media) were treated as groups and these groups were compared for differential expression levels. Bioinformatics Core personnel concatenated the single-read data files into a single ".fastq" formatted file for each sample. They checked the quality of the raw reads data for each sample using FastQC Version 0.10.0 (http://www.bioinformatics.bbsrc.ac.uk/projects/fastqc/) to identify features of the data that may indicate quality problems (e.g. low quality scores, over-represented sequences, inappropriate GC content, etc.). They used the software package Tuxedo Suite for alignment, differential expression analysis, and post-analysis diagnostics [97]-[99]. Briefly, they aligned reads to the reference genome (ENSEMBL E. Coli K12MG1655, from: http://bacteria.ensembl.org) using Bowtie Version 2.1.0.0. They used default parameter settings for alignment, with the exception of "--b2-very-sensitive" telling the software to spend extra time searching for valid alignments. In addition, they used FastQC for a second round of quality control (post-alignment), to ensure that only high quality data would be 
input to expression quantitation and differential expression analysis. They used Cufflinks/CuffDiff (version 2.1.1) for expression quantitation and differential expression analysis, using ENSEMBL E. Coli K12MG1655.fa as the reference genome sequence and ENSEMBL E. Coli K12MG1655.gtf as the reference transcriptome annotation. For this analysis, they used parameter settings: "--multi-read-correct" to adjust expression calculations for reads that map in more than one locus, as well as "--compatible-hits-norm" and "--upper-quartile -norm" for normalization of expression values. They generated diagnostic plots using the CummeRbund package. They used locally developed scripts to format and annotate the differential expression data output from CuffDiff. Briefly, they identified genes and transcripts as being differentially expressed based on three criteria: test status $=$ "OK", FDR $<0.05$ and fold change $\geq 1.5$. They annotated genes and isoforms with ENSEMBL gene IDs.

We used Bioinformatics Core results to determine lists of differentially expressed genes in comparisons "WT vs Mut in Control", "WT vs Mut in Waste", "WT Control vs WT in Waste", and "Mut Control vs Mut Waste", where WT and Mut refer to parent strain K12 MG1655 and evolved strain K2C2, respectively, and Control and Waste refer to Complete MOPS media and $30 \mathrm{vol} \%$ AqAl media, respectively. So, there were two comparisons of different strains grown in the same media, and two comparisons of the same strain growing in different media. The lists of differentially expressed genes were divided into up and down regulated genes. For example, the list of "up" genes in the "WT vs Mut in Waste" comparison are those that strain K2C2 expresses more than parent strain K12 in 30 vol\% AqAl medium.

We individually loaded these gene lists into the EcoCyc E. coli genome and metabolic pathway database "SmartTable" web tool (http://ecocyc.org/groups?orgid=ECOLI). We used this tool to perform an "enrichment analysis" to determine metabolic pathways that were statistically overrepresented in the lists. This was performed using Fischer Exact analysis with Parent-Child Union and a cut-off for significance at a p-value less than 0.1. Parent-Child Union correction is a common tool for reducing false positives when enriching lists of genes with multiple references in hierarchical structures, like pathway or gene ontology systems [100]. The enriched pathway lists for the "WT vs Mut Waste" comparison 
were crossreferenced to the lists for "WT vs Mut Control" to determine which pathways differentially expressed between $\mathrm{K} 2 \mathrm{C} 2$ and parent $\mathrm{K} 12$ in AqAl medium were also differentially expressed in Complete MOPS medium. In the same fashion, enriched pathway lists for "Mut Control vs Mut Waste" were compared to that of "WT Control vs WT Waste" to determine which pathways differentially expressed in K2C2 between Complete MOPS and AqAl media were also differentially expressed in strain $\mathrm{K} 12$ between the different media. 
Table 4.4 Selection data for Strains of Interest from Sequencing Candidates

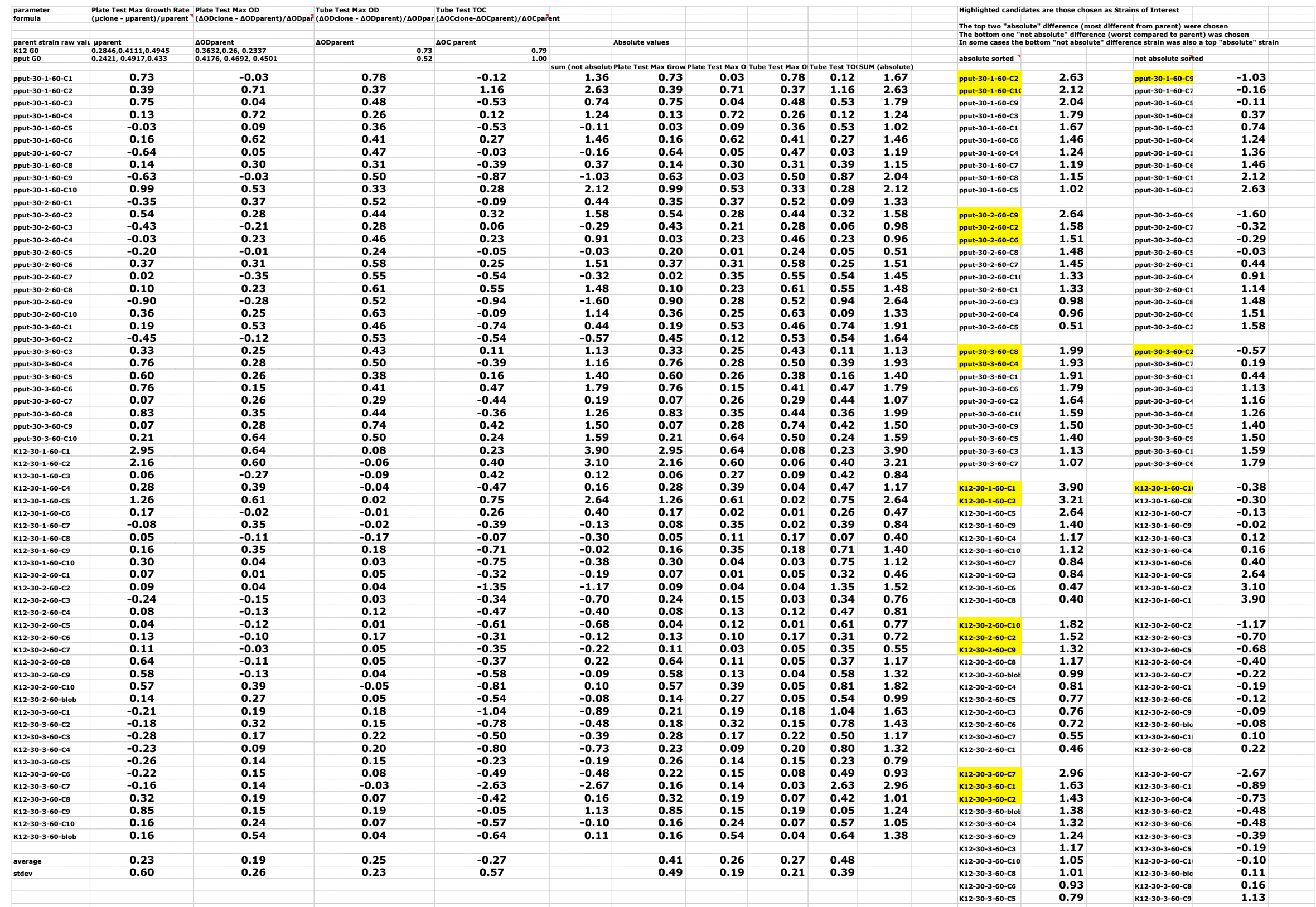


Table 4.5 Primer and oligomer design

Primers designed for amplification of $16 \mathrm{~S}$ gene for species confirmation via Sanger Sequencing

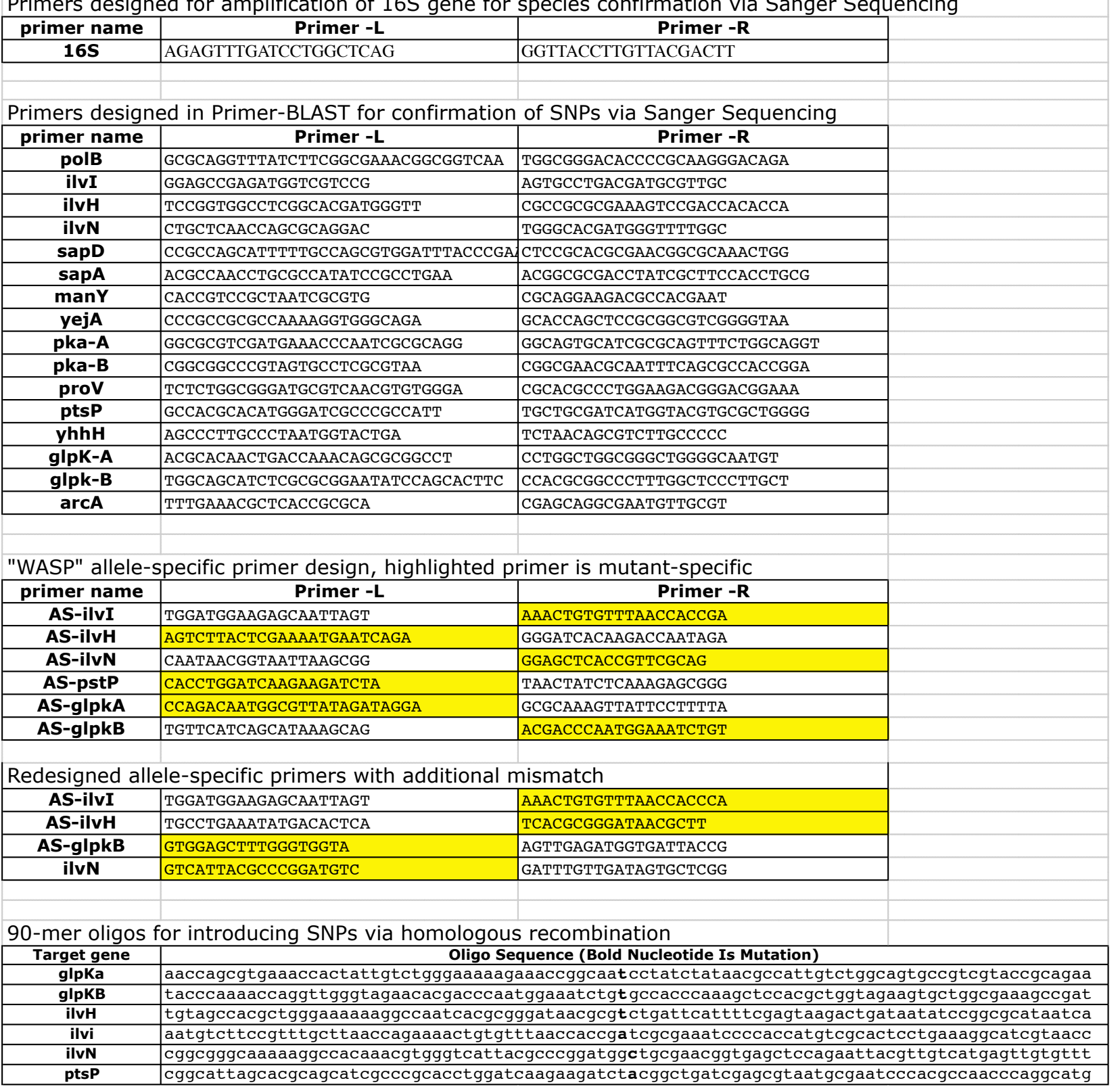




\section{Part III}

\section{Examining the Utility of a Microbial Side-Culture: Hydrothermal Liquefaction of Microbial Biomass and Regrowth of Algae on Bacteria-processed AqAl}

\section{Chapter 5 \\ Hydrothermal Liquefaction of Bacteria and Yeast Monocultures}

\subsection{Summary}

We hydrothermally treated monocultures of Escherichia coli, Pseudomonas putida, Bacillus subtilis, and Saccharomyces cerevisiae at isothermal $\left(350{ }^{\circ} \mathrm{C}\right.$ for $\left.60 \mathrm{~min}\right)$ and at fast (rapid heating for $1 \mathrm{~min}$ ) liquefaction conditions. Fast hydrothermal liquefaction (HTL) of P. putida and S. cerevisiae produced the highest biocrude yields of $47 \pm 13$ wt $\%$ and $48 \pm 9$ wt \%, respectively. Biocrudes generated via fast hydrothermal liquefaction were always richer in $\mathrm{O}$ and $\mathrm{N}$ and had a higher yield of hexane-insoluble products. Isothermal HTL of all microorganisms always produced an aqueous phase richer in $\mathrm{NH}_{3}$ than the aqueous phase from fast HTL. Up to $62 \pm 9 \%$ of the chemical energy in the biomass could be recovered in the biocrude product fraction. These results demonstrate the feasibility of applying HTL to produce high yields of biocrude from bacteria and yeast that are high in protein (> $80 \mathrm{wt} \%$ d.a.f.) and low in lipids (<3 wt \% d.a.f.) Such microorganisms could serve as a renewable feedstock for biofuels.

The entirety of the material presented in this section was published in Energy and Fuels as follows: Peter J. Valdez, Michael C. Nelson, Julia L. Faeth, Henry Y. Wang, Xiaoxia Nina Lin, and Phillip E. Savage. 2013. "Hydrothermal liquefaction of bacteria and yeast monocultures". Energy and Fuels, 28, 67-75.

Michael Nelson, the $2^{\text {nd }}$ author, was responsible for the growth and concentration of microbial biomass and analysis of the aqueous phase generated from the HTL reactions. 
Peter Valdez, the $1^{\text {st }}$ author, was responsible for performing "isothermal HTL" reactions, separating the products, and analyzing the solids, gasses, and oil formed. Julia Faeth was responsible for performing "fast HTL" reactions, separating the products, and analyzing the solids, gasses, and oil formed. Material from this publication is being used with permission from the authors. 


\subsection{Introduction}

Bacteria and yeasts are widely used in industrial biochemical processes that convert feedstock substrates into finished products such as specialty chemicals, [50] food products, [101] and biofuels. [102]-[105]. Although there is significant value in the aforementioned products, the microbial biomass itself is usually discarded as a waste. Biochemical processes are also used in wastewater treatment and they create a secondary sludge that is rich in microorganisms, which are often collected and discarded. [106] This microbial biomass can have heating values comparable to that of microalgae, which has received tremendous attention as a feedstock for biofuels. Hydrothermal liquefaction (HTL), a hightemperature and high-pressure process, can convert whole wet biomass into energy-dense bio-oils, also referred to as biocrude. [7], [11], [18], [107] HTL of bacteria and yeasts may create an opportunity to produce a renewable biofuel from low-value materials.

The hydrothermal liquefaction of microbial communities in sludge is not new. HTL of sludge from biologically treated cornstarch and pulp/paper waste resulted in biocrude yields of 15 - 30 wt \% and 42 - 65 wt \%, respectively. [108], [109] HTL of anaerobically digested sludge from municipal sources has been less successful, producing biocrude yields of $\leq 10 \mathrm{wt} \%$ [110], [111].

Recent studies of HTL of microorganisms have focused on microalgae as a feedstock and produced biocrude yields as high as $66 \mathrm{wt} \%$ [7], [11], [18], [58], [112], [113] Although a majority of the algal biomass is converted into biocrude, the aqueous co-product also contains some organic carbon along with nutrients such as nitrogen- and phosphoruscontaining substrates. This aqueous co-product can be utilized for cultivating additional biomass. Using it to grow more algae can be difficult as several studies have shown that it can be toxic or nutrient-limited. [14], [16], [17], [68] Moreover, recycling this water, which contains organic carbon, to an open pond for algae growth may substantially increase the risk of invasion of contaminating heterotrophs into the algae culture. However, Nelson et al. have recently demonstrated the feasibility of cultivating Escherichia coli and Pseudomonas putida monocultures utilizing this aqueous-phase with minimal dilution and nutrient supplementation [77]. Adding a microbial cultivation step in an algae HTL biorefinery to utilize the organic carbon in the aqueous phase can enhance ease of its recycling to an open pond and provide additional biomass for hydrothermal liquefaction. 
To the best of our knowledge, HTL of microbial monocultures, such as bacteria and yeast, has not been examined.

We cultivated Escherichia coli, Pseudomonas putida, Bacillus subtilis, and Saccharomyces cerevisiae and subjected the biomass to hydrothermal treatment at fast (rapid heating for $1 \mathrm{~min}$ ) and isothermal $\left(350^{\circ} \mathrm{C}, 60 \mathrm{~min}\right.$ ) liquefaction conditions. E. coli and $S$. cerevisiae are bacterial and yeast species, respectively, that are frequently used in industrial bioprocesses. We selected P. putida because it is known to metabolize a diverse array of substrates, which makes it a good candidate for growth on complex waste streams [71]. B. subtilis, a widely studied Gram-positive bacterium, [114] was included to investigate the impact of its differing cellular composition (particularly in the peptidoglycan-abundant cell wall) on liquefaction products when compared to the other two Gram-negative bacteria (E. coli and P. putida). We report herein the results of how microorganism selection, growth media, cellular structure (Gram-positive vs. Gramnegative), and hydrothermal treatment conditions affect the yield and composition of the different product fractions from HTL.

\subsection{Overview}

We grew Gram-negative bacteria E. coli, and P. putida, Gram-positive bacterium $B$. subtilis, and yeast $S$. cerevisiae in rich medium. We also grew E. coli in a chemically defined minimal medium. We concentrated these cultures into slurries for hydrothermal liquefaction reactions under both "conventional HTL" conditions of $350^{\circ} \mathrm{C}$ for 60 minutes and "fast HTL" conditions of $600{ }^{\circ} \mathrm{C}$ for one minute $\left(600{ }^{\circ} \mathrm{C}\right.$ being the temperature of the ambient environment around the reactor, with internal temperatures not exceeding 400 ${ }^{\circ} \mathrm{C}$ ). We separated biocrude oil, aqueous, gas, and solids products and analyzed them to determine overall yield, energy yield, elemental composition, and molecular composition. Biocrude oil yields for most of the microbial species were found to be comparable to those published for microalgae species.

\subsection{Results and Discussion}

This section first reports the characteristics of the microorganisms we cultivated and then reports the results of the hydrothermal treatment of the biomass. The latter section describes the yield, elemental composition, and selected molecular composition of 
the product fractions. We also report the heating value and energy recovery of the biocrudes and compare results among the various microorganisms.

\subsubsection{Feedstock Analysis}

Table 5.1 Elemental (wt \%), biochemical composition (wt \%), and HHV (MJ/kg) of the biomass

\begin{tabular}{|c|c|c|c|c|c|c|c|c|c|c|}
\hline & $\mathrm{C}$ & $\mathrm{H}$ & $\mathrm{N}$ & S & 0 & ash & lipid & $\begin{array}{l}\text { protei } \\
\mathrm{n}\end{array}$ & $\begin{array}{l}\text { carbohydrat } \\
\text { e }\end{array}$ & HHV \\
\hline E. coli TB & 46.54 & 6.69 & 13.70 & 0.67 & 25.58 & $\begin{array}{c}6.82 \\
\pm \\
0.02\end{array}$ & $\begin{array}{c}0.57 \pm \\
0.36\end{array}$ & 86 & 7 & 22 \\
\hline E. coli MM & 47.32 & 6.88 & 13.17 & 0.58 & 27.15 & $\begin{array}{c}4.9 \pm \\
0.1\end{array}$ & $\begin{array}{c}2.6 \pm \\
0.1\end{array}$ & 82 & 10 & 23 \\
\hline P. putida & 46.58 & 7.08 & 13.23 & 0.55 & 21.48 & 11 & $\begin{array}{c}2.7 \pm \\
0.7\end{array}$ & 83 & 4 & 23 \\
\hline B. subtilis & 42.65 & 6.56 & 11.45 & 0.43 & 25.91 & $\begin{array}{r}13.0 \\
\pm 0.4\end{array}$ & $\begin{array}{c}0.55 \pm \\
0.03\end{array}$ & 72 & 15 & 21 \\
\hline S. cerevisiae & 46.47 & 7.31 & 12.04 & 0.47 & 29.03 & $\begin{array}{c}4.68 \\
\pm \\
0.01\end{array}$ & $\begin{array}{c}2.7 \pm \\
0.6\end{array}$ & 75 & 17 & 22 \\
\hline
\end{tabular}

Table 5.1 shows the elemental and biochemical content of each of the biomass feedstocks. There are but modest variations in the elemental composition of $\mathrm{C}, \mathrm{H}, \mathrm{N}$, and S. The $\mathrm{C}$ and $\mathrm{N}$ wt $\%$ for $E$. coli are within $6 \%$ relative difference of those reported previously [115], [116]. Although the two E. coli cultures were cultivated using different growth media their $\mathrm{C}, \mathrm{H}, \mathrm{N}$, and $\mathrm{O}$ contents were within $10 \%$ of each other on a relative basis. B. subtilis and P. putida had the highest ash compositions of 13 and $11 \mathrm{wt} \%$, respectively, whereas the other organisms had $<7 \mathrm{wt} \%$ ash content.

The E. coli grown with minimal media, S. cerevisiae, and P. putida had the highest lipid contents, but they were all $\leq 2.7 \mathrm{wt} \%$. E. coli grown with terrific broth and B. subtilis had lipid values $<0.6$ wt $\%$. The E. coli TB was grown in a nutrient-rich condition so it is 
reasonable that the cells would accumulate less lipids than the E. coli MM. Likewise, Grampositive organisms such as $B$. subtilis have fewer lipids possibly due to the lack of an outer membrane in the cell envelope compared to Gram-negative organisms. The carbohydrate content of each biomass sample varied between 4 and $17 \mathrm{wt} \%$. All biomass samples were rich in protein ( $\geq 72 \mathrm{wt} \%$ ). Compared to microalgae feedstocks typically subjected to HTL, the yeast and bacteria have a much lower lipid content and much higher protein and $\mathrm{N}$ content [117].

\subsubsection{Yields of Product Fractions}

As a control experiment we exposed dried, unreacted biomass to fresh solvents at room temperature. After exposure to dichloromethane and water, $\leq 4 \mathrm{wt} \%$ of the biomass partitioned to the organic phase as biocrude. The remaining biomass partitioned to the solid product fraction. Therefore, solvent extraction alone does not generate the high biocrude yields that are typical of hydrothermal treatment.

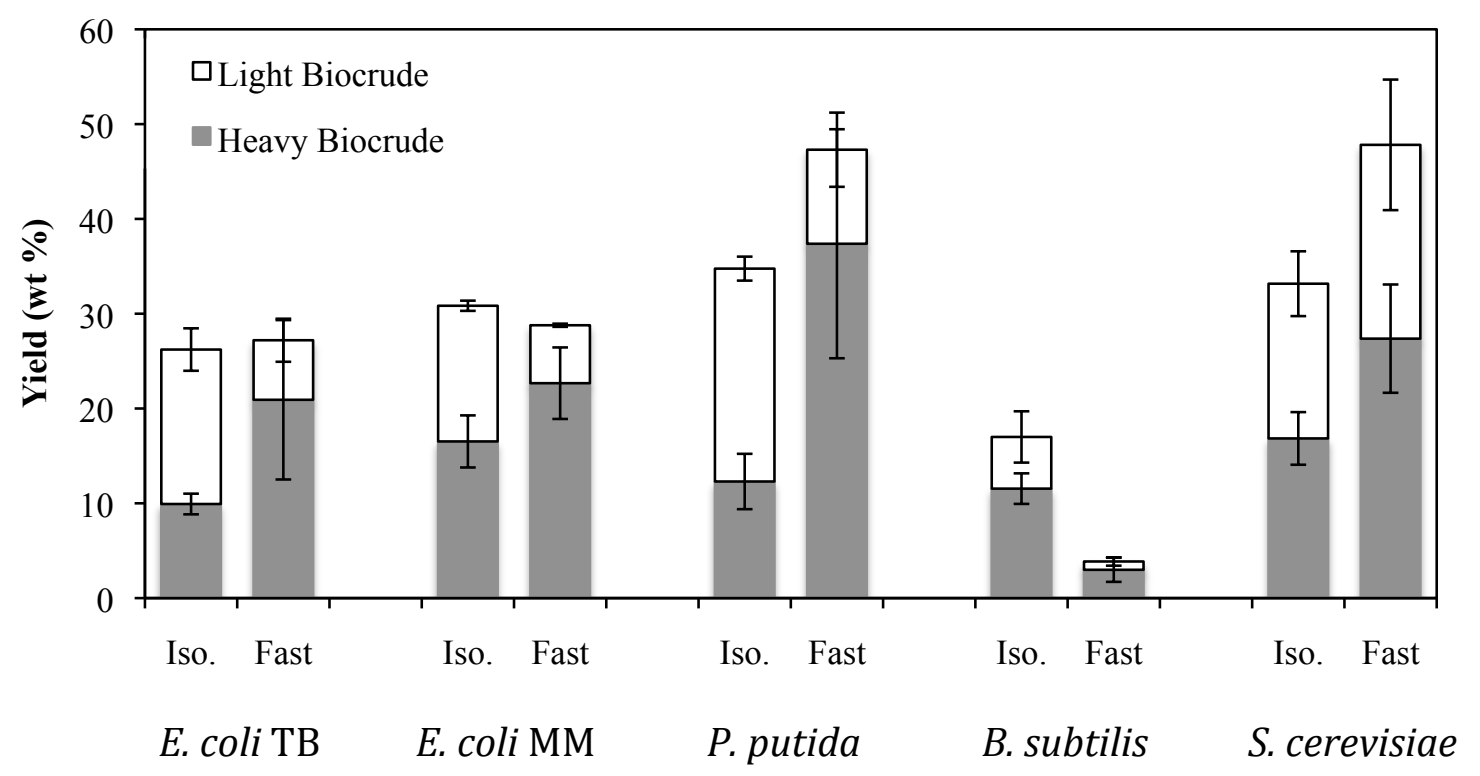

Figure 5.1 Yields of light and heavy biocrude product fractions (wt \% daf) for each biomass and isothermal and fast HTL

Figure 5.1 shows the dry ash-free (daf) yields of light and heavy biocrudes for each organism at both hydrothermal treatment conditions. Yields of biocrudes are presented on a dry basis in Table 5.1 of the supplemental information. The isothermal treatment at 350 ${ }^{\circ} \mathrm{C}$ for $60 \mathrm{~min}$ is common practice [7], [10], [18]-[20] so the present results from the yeast 
and bacterial biomass can be compared with those from HTL of other feedstocks. We also used rapid heating or fast HTL, which can increase the biocrude yield [113]. The average maximum temperature observed in the dummy reactor during fast HTL was $276 \pm 41^{\circ} \mathrm{C}$, and the average heating rate of the reactors at fast liquefaction conditions was $216 \pm 37$ ${ }^{\circ} \mathrm{C} / \mathrm{min}$, with the uncertainty representing one standard deviation of the population.

$B$. subtilis showed the lowest total yield of biocrude for both conventional isothermal ( $17 \pm 3 \mathrm{wt} \% \mathrm{daf}$ ) and fast ( $4 \pm 1 \mathrm{wt} \%$ daf) liquefaction conditions. These low yields may be linked to its cellular structure. One contributing factor may be the different molecular composition of the cell envelope. Gram-positive bacteria, including B. subtilis, have a thick layer of peptidoglycans, which are polysaccharides cross-linked by polypeptides. These biomacromolecules likely hydrolyze to simple sugars and amino acids that would reside within the aqueous-phase product fraction. Past research has shown that continued hydrothermal processing of water-soluble amino acids and carbohydrates can form organic-solvent soluble products [54]. The 1 min reaction time for fast HTL may have been long enough to form these water-soluble products, yet too brief to allow their repolymerization into biocrude components. Such processes could account for the higher biocrude yield at isothermal HTL conditions observed in Figure 5.1 for B. subtilis.

Fast hydrothermal treatment of $P$. putida and $S$. cerevisiae produced the most biocrude with yields of $47 \pm 13$ wt $\%$ and $48 \pm 9$ wt \%, respectively. The higher yield of biocrude from $S$. cerevisiae may also be linked to the cell structure of the yeast, in this case improving the yield, unlike the result from B. subtilis.

The larger standard deviation in the yields from fast HTL probably arises from the lower biomass loadings. There is less material to recover in these experiments and transfer losses become more significant, on a relative basis, compared to conventional liquefaction [113]. The reaction conditions did not affect the total yield of biocrude for both types of $E$. coli. Figure 5.1 shows that fast liquefaction always produced a higher fraction of heavy biocrude than light biocrude, regardless of organism. This trend is also true for the fast liquefaction of the microalga Nannochloropsis sp [113]. Figure 5.1 shows that, with the exception of $B$. subtilis, the yield of total biocrude produced at conventional HTL conditions did not vary much, ranging between 26 - 35 wt \% daf for the different microorganisms. 
Table 5.2 Yields of Solid, Aqueous-Phase, and Gas Product Fractions (wt \% dry basis)

\begin{tabular}{|c|c|c|c|c|c|c|}
\hline & \multicolumn{2}{|c|}{ Solids } & \multicolumn{2}{|c|}{ Aqueous-phase Products } & \multicolumn{2}{|c|}{ Gas } \\
\hline & Iso. & Fast & Iso. & Fast & Iso. & Fast \\
\hline E. coli $\mathrm{TB}$ & $2.1 \pm 0.5$ & $8.5 \pm 4.3$ & $72 \pm 2$ & $66 \pm 9$ & $\begin{array}{c}1 \pm 0.1 \\
1.2 \pm\end{array}$ & -1 \\
\hline E. coli MM & $5.0 \pm 0.6$ & $26 \pm 3$ & $64 \pm 3$ & $46 \pm 5$ & $\begin{array}{c}0.7 \\
3.8 \pm\end{array}$ & 0.30 \\
\hline P.putida & $1.8 \pm 0.6$ & $4.4 \pm 2.0$ & $64 \pm 3$ & $52 \pm 11$ & 1.7 & 1.7 \\
\hline B. subtilis & $3.6 \pm 0.6$ & $3.4 \pm 1$ & $81 \pm 3$ & $92 \pm 2$ & $\begin{array}{c}1 \pm 0.4 \\
1.9 \pm\end{array}$ & 1.8 \\
\hline S. cerevisiae & $4.6 \pm 1.1$ & $5.6 \pm 0.3$ & $62 \pm 4$ & $43 \pm 9$ & 0.5 & 6.9 \\
\hline
\end{tabular}

Table 5.2 shows the yield of solids (water- and dichloromethane-insoluble), aqueous-phase-products, and gas for both isothermal and fast HTL of each organism. The aqueous phase was translucent amber in color for all of the different microorganisms. More than 42 wt \% of the biomass is converted to aqueous-phase products. The solids were gray powders that settled at the bottom of the test tube. Solid yields were always $<9$ wt \% with the exception of E. coli MM treated with fast HTL. E. coli differentially expresses genes that affect cell structure when grown in minimal versus rich medium [118]. The milder reaction conditions in fast HTL may not have been sufficient to break down certain components in the $E$. coli cell, especially when grown in minimal media. The data in Table 5.2 suggest that the residual solids remaining after fast liquefaction likely form mostly aqueous-phase products when reacted under the harsher isothermal HTL conditions.

Table 5.2 shows that $<7$ wt $\%$ of the biomass is converted to gas. The differences in the composition of the gas products from HTL of each biomass were not statistically significant. Therefore, Figure 5.2 shows the average composition of the gas phase from 
isothermal and fast HTL. The gas phase from isothermal HTL was on average $93 \pm 6 \mathrm{~mol} \%$ $\mathrm{CO}_{2}$ with the balance being $\mathrm{H}_{2}, \mathrm{CO}, \mathrm{CH}_{4}, \mathrm{C}_{2} \mathrm{H}_{4}$, and $\mathrm{C}_{2} \mathrm{H}_{6}$. The gas from fast HTL had significantly less $\mathrm{CO}_{2}, 64 \pm 15 \mathrm{~mol} \%$, and significantly more $\mathrm{CO}, \mathrm{CH}_{4}, \mathrm{C}_{2} \mathrm{H}_{4}$, and $\mathrm{C}_{2} \mathrm{H}_{6}$. At harsher reaction conditions, $\mathrm{CO}_{2}$ is a common product from the hydrothermal decomposition of amino acids [119].

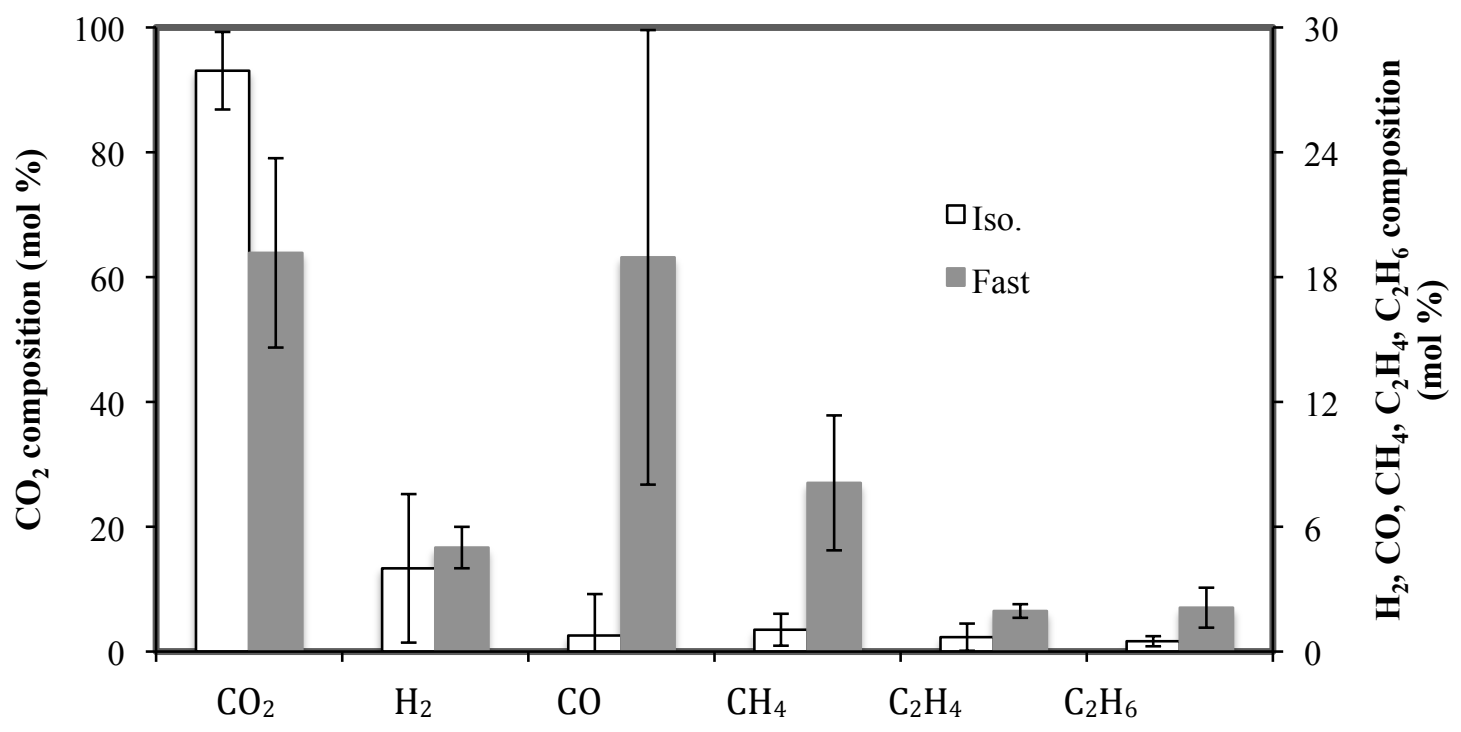

Figure 5.2 Average composition of the gas phase from isothermal and fast HTL

\subsubsection{Elemental Composition of the Light and Heavy Biocrudes}

Figure 5.3 shows the $\mathrm{C}, \mathrm{N}, \mathrm{O}$, and $\mathrm{S}$ content (wt \%) of the light and heavy biocrudes for each organism (exact values are included in the supplemental information). Elemental ratios of $\mathrm{H}: \mathrm{C}, \mathrm{N}: \mathrm{C}, \mathrm{O}: \mathrm{C}$, and $\mathrm{S}: \mathrm{C}$ in the light and heavy biocrudes are presented in the supplemental information. Figure 5.3a shows that regardless of the conditions of the hydrothermal treatment, the light biocrude always had a higher wt $\% \mathrm{C}$ than the heavy biocrude, similar to the results obtained previously for HTL of Nannochloropsis sp [11], [113]. Fast HTL biocrudes, both light and heavy, always had a lower wt \% $\mathrm{C}$ than the biocrudes from conventional isothermal liquefaction. Figure 5.3b Figure 5.3c show that the light and heavy biocrudes from fast HTL are always richer in $\mathrm{N}$ and $\mathrm{O}$ content than their counterparts from isothermal HTL. Previous results for HTL of Nannochloropsis sp. showed that increasing the reaction severity, that is increasing holding time and/or increasing reaction temperature, reduces the 0 content in the biocrude [11]. The results in Figure 5.3c 
show the same trend. Even so, the $\mathrm{N}$ and $\mathrm{O}$ content in these biocrudes are roughly an order of magnitude greater than those in most petroleum crudes, [120] and may present challenges to upgrade the biocrude to a hydrocarbon fuel. All of the biocrudes, however, have reduced 0 content compared to the original biomass.

Figure 5.3d shows that the $\mathrm{S}$ content in the biocrude varies among the different organisms, but it is always $<1 \mathrm{wt} \%$ putting it within range of the $S$ content of most petroleum crudes [120]. The heavy biocrude from fast liquefaction is always richer in $S$ than the heavy biocrude from conventional liquefaction. The $\mathrm{S}$ content in the light biocrude from $S$. cerevisiae treated by fast liquefaction was the lowest at 0.15 wt $\%$. 
(a)

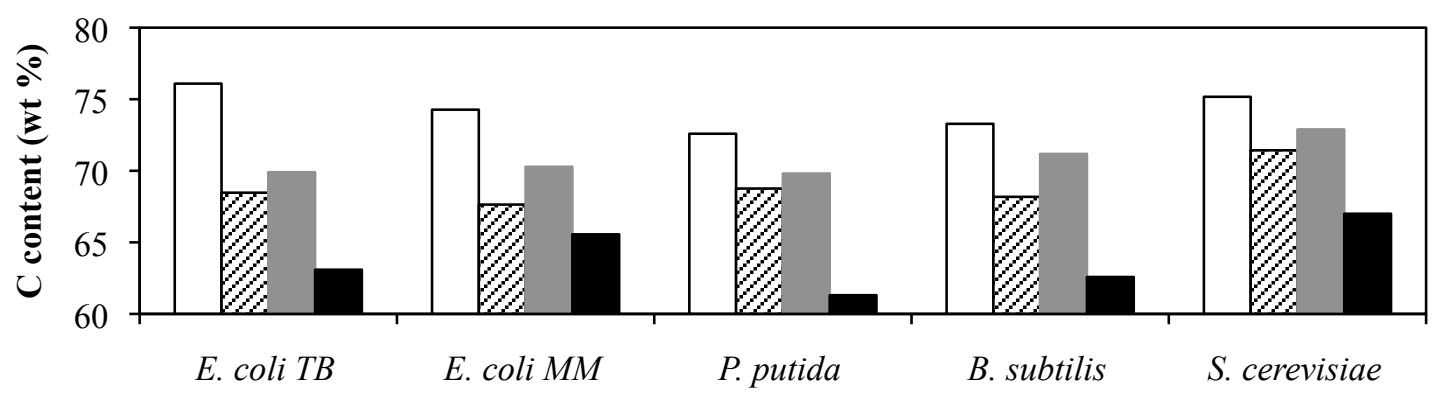

(b)

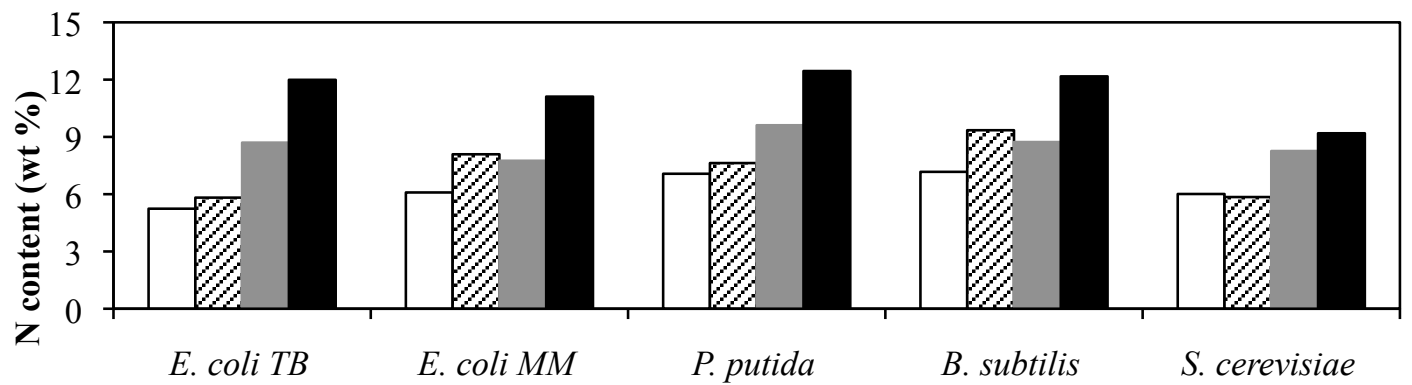

(c)

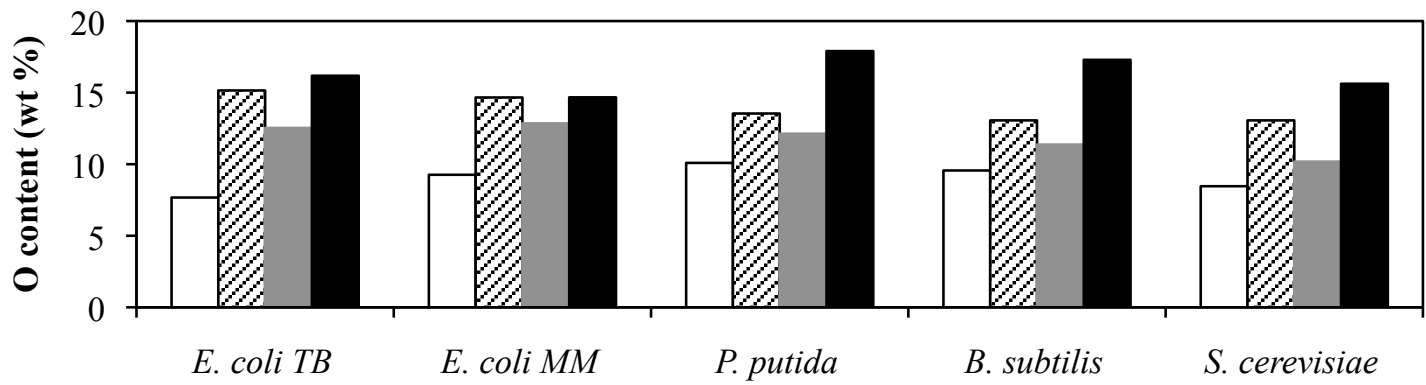

(d)

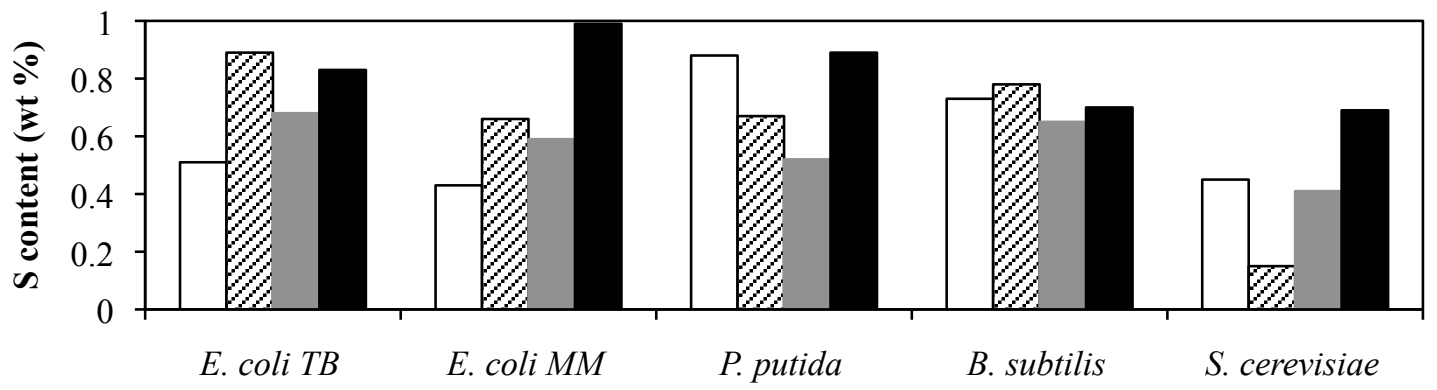

$\square$ Light Biocrude, Iso. $⿴$ L Light Biocrude, Fast $\square$ Heavy Biocrude, Iso.

Figure 5.3 Composition of a) C, b) $N$, c) 0 , and d) $S$ in the light and heavy biocrudes 


\subsubsection{Ammonia in the Aqueous Phase}

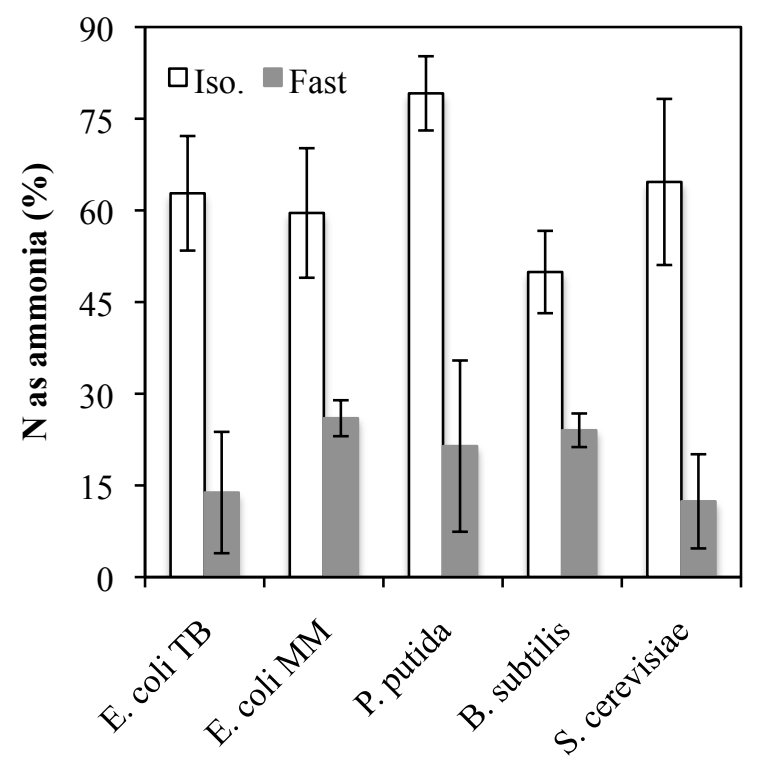

Figure 5.4 Percentage of $\mathrm{N}$ as ammonia in the aqueous phase

Figure 5.4 shows the percentage of the total $\mathrm{N}$ from the biomass that is present as dissolved ammonia in the aqueous phase. Isothermal HTL of the biomass favors the near complete conversion of water-soluble $\mathrm{N}$ products into ammonia. As reaction severity increases more of the $\mathrm{N}$ in the biomass is converted into ammonia [11]. Converting $\mathrm{N}$ containing molecules into dissolved ammonia makes the nitrogen more bioavailable for use by most microorganisms. This conversion facilitates the recycling of $\mathrm{N}$ in a biorefinery, which is an important consideration for environmental sustainability.

\subsubsection{Elemental Distribution}

Knowing the gravimetric yields and the elemental composition of each product fraction allows one to calculate how the various elements are distributed among the product fractions. Carbon and nitrogen are of most interest since the C content strongly influences heating value and $\mathrm{N}$ recycling is essential for a sustainable hydrothermal biorefinery. The present results show that at most $64 \%$ of the $\mathrm{C}$ in the biomass goes to the biocrude. B. subtilis had the lowest C distributed to the biocrude while P. putida and $S$. cerevisiae had the highest distribution of $\mathrm{C}$ because of their higher yields of biocrude. 
Similar to the results from the HTL of microalgae, the majority of the N ( $80 \%$ or more for conventional isothermal HTL) resides in the aqueous phase and solids [11]. This outcome is desirable as it facilitates recycling the $\mathrm{N}$-containing compounds as nutrients for cultivation of additional biomass. Less than $41 \%$ of the total $\mathrm{N}$ from the biomass is distributed to the biocrude.

\subsubsection{Heating Value and Energy Recovery}

Table 5.3 Higher Heating Value and Energy Recovery of the Biocrude

\begin{tabular}{|c|c|c|c|c|}
\hline \multirow[t]{2}{*}{ Organism } & \multirow[t]{2}{*}{ HTL } & \multicolumn{2}{|c|}{ HHV (MJ/kg) } & \multirow{2}{*}{$\begin{array}{c}\text { Energy Recovery } \\
\text { in the Biocrude } \\
(\%)\end{array}$} \\
\hline & & $\begin{array}{c}\text { Light } \\
\text { Biocrude }\end{array}$ & $\begin{array}{c}\text { Heavy } \\
\text { Biocrude }\end{array}$ & \\
\hline \multirow[t]{2}{*}{ E. coli TB } & Iso. & 38 & 33 & $43 \pm 3$ \\
\hline & Fast & 34 & 30 & $38 \pm 8$ \\
\hline \multirow[t]{2}{*}{ E. coli $\mathrm{MM}$} & Iso. & 37 & 34 & $48 \pm 4$ \\
\hline & Fast & 33 & 31 & $40 \pm 5$ \\
\hline \multirow[t]{2}{*}{ P. putida } & Iso. & 36 & 33 & $52 \pm 6$ \\
\hline & Fast & 34 & 29 & $62 \pm 10$ \\
\hline \multirow[t]{2}{*}{ B. subtilis } & Iso. & 36 & 34 & $29 \pm 6$ \\
\hline & Fast & 33 & 29 & $6 \pm 2$ \\
\hline \multirow{2}{*}{$\begin{array}{l}\text { S. } \\
\text { cerevisiae }\end{array}$} & Iso. & 37 & 35 & $53 \pm 10$ \\
\hline & Fast & 35 & 31 & $60 \pm 25$ \\
\hline
\end{tabular}

Table 5.3 shows the higher heating values of the light and heavy biocrudes and the percentage of the chemical energy in the biomass that is recovered in the biocrude. The heating value of the light biocrude was always higher than that of the heavy biocrude and 
biocrudes produced at isothermal HTL conditions had higher heating values than biocrudes produced at fast HTL conditions. These trends are simply a manifestation of the trends in the $\mathrm{C}$ and $\mathrm{O}$ content of the various biocrude fractions. Regardless of the biomass feedstock processed, the variation in heating value for a given biocrude (light or heavy) fraction from a given HTL approach (isothermal or fast) is always $<2 \mathrm{MJ} / \mathrm{kg}$. It appears that the processing conditions and product fractionation protocol play a larger role in determining heating value than does the biomass feedstock [20].

The last column of Table 5.3 shows the total energy recovered in the biocrude from the original biomass. S. cerevisiae and P. putida had the highest energy recovery values for both hydrothermal treatment conditions. B. subtilis had the lowest energy recovery (recall that it gave the lowest yields of biocrude). The energy recoveries in HTL biocrude from bacteria and yeast are not as high as those often observed from HTL of microalgae where values exceeded $70 \%$ at the same processing conditions [11], [113]. Of course microalgae typically have lipid contents that are an order of magnitude higher than those in the bacteria and yeast used in this study. Higher lipid contents tend to correlate with higher biocrude yields [7] and higher energy recoveries in the biocrude. Nevertheless, Table 5.3 shows that HTL can produce energy-dense biocrudes containing $40 \%$ or more of the chemical energy in the microbial biomass.

\subsubsection{Molecular Composition of the Light Biocrude}

We analyzed the light biocrude using GC-MS. Table 5.4 shows the components in the light biocrude, for which the GC-MS software gave at least a > $50 \%$ match factor with a compound in its mass spectra library, and their relative abundance quantified by peak area. Not all of the compounds in the biocrude could be identified because of the large number of low intensity peaks present in each chromatogram. All chemical identities in Table 5.4 remain tentative, as we used no authentic standards to get positive identities. The peak areas listed in Table 5.4 provide a qualitative representation of the relative abundance of that compounds in the light biocrude.

Table 5.4 shows that the fast HTL biocrude was less likely to contain heterocycles and aromatics. The nitrogen-containing heterocycles such as substituted indoles and 
amines possibly derived from the decomposition of porphyrins or the proteins that are abundant in the microorganisms [121]. The nitrogen-containing heterocycles are not uncommon in petroleum crude, but the presence of such compounds in higher concentrations in the biocrude make it more difficult to upgrade to a hydrocarbon fuel. Free fatty acids appear in Table 5.4, and these are also common products in biocrudes from microalgae HTL [18]. For the microbial biomass studied here they are likely to be mostly derived from the cell membrane. The fast HTL biocrudes contain a higher percentage of fatty acids than the biocrudes from isothermal HTL, potentially showing that lipids in the cell hydrolyze faster than the other biomolecules. Fatty acid amides are more common in the isothermal HTL products, suggesting that the higher concentration of $\mathrm{NH}_{3}$ present in the reactor facilitates replacing the hydroxyl group in the fatty acid. Table 5.4 shows that more of the cyclic proline dimer [122], which is likely a decomposition product of proteins, is found in the fast HTL biocrude, suggesting the incomplete decomposition of proteins to amino acids at milder conditions.

Table 5.4 Tentative identities and relative abundance of different compounds in the light biocrude

\begin{tabular}{|c|c|c|c|c|c|c|c|c|c|c|c|}
\hline \multirow{3}{*}{ Name } & \multirow{3}{*}{ Structure } & \multicolumn{10}{|c|}{$\%$ Total Area } \\
\hline & & \multicolumn{2}{|c|}{ E. coli $\mathrm{TB}$} & \multicolumn{2}{|c|}{ E. coli MM } & \multicolumn{2}{|c|}{ P.putida } & \multicolumn{2}{|c|}{ B. subtilis } & \multicolumn{2}{|c|}{ S. cerevisiae } \\
\hline & & Iso. & Fast & Iso. & Fast & Iso. & Fast & Iso. & Fast & Iso. & Fast \\
\hline 4-methylphenol & & 2.43 & -1 & - & - & 1.82 & - & 5.7 & - & 1.14 & - \\
\hline 4-ethylphenol & & 1.88 & - & 1.4 & - & 1.41 & - & 4.09 & - & 0.58 & - \\
\hline piperidin-2-one & & 0.8 & - & 0.44 & - & 0.79 & - & 2.72 & - & 0.29 & - \\
\hline $\begin{array}{l}\text { hexahydro-2H- } \\
\text { azepin-2-one }\end{array}$ & & 0.81 & - & - & - & 0.56 & - & 2.66 & - & 0.31 & - \\
\hline indole & & 0.75 & - & 0.49 & 1.32 & 0.57 & 1.04 & 1.65 & 1.78 & 0.12 & - \\
\hline
\end{tabular}




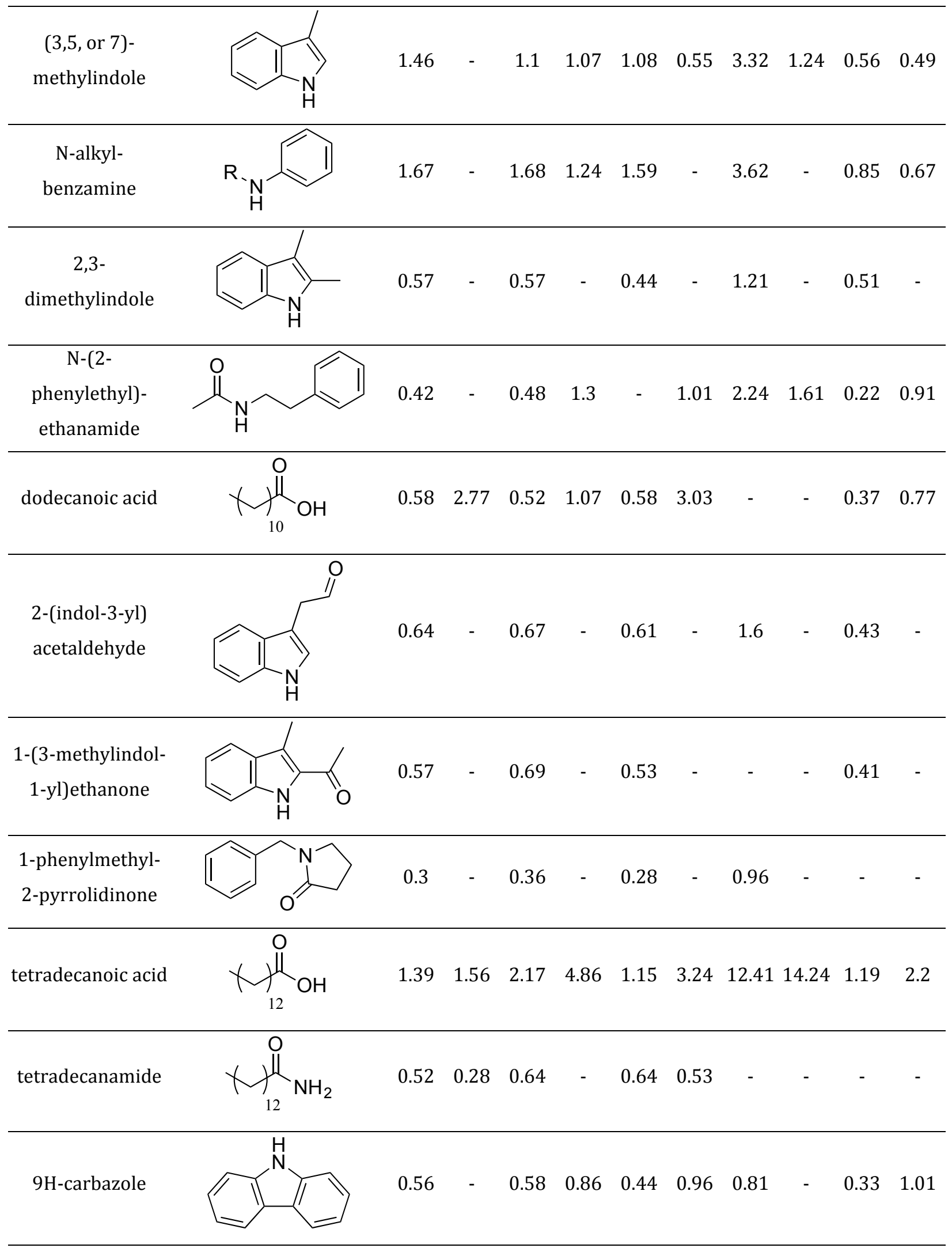




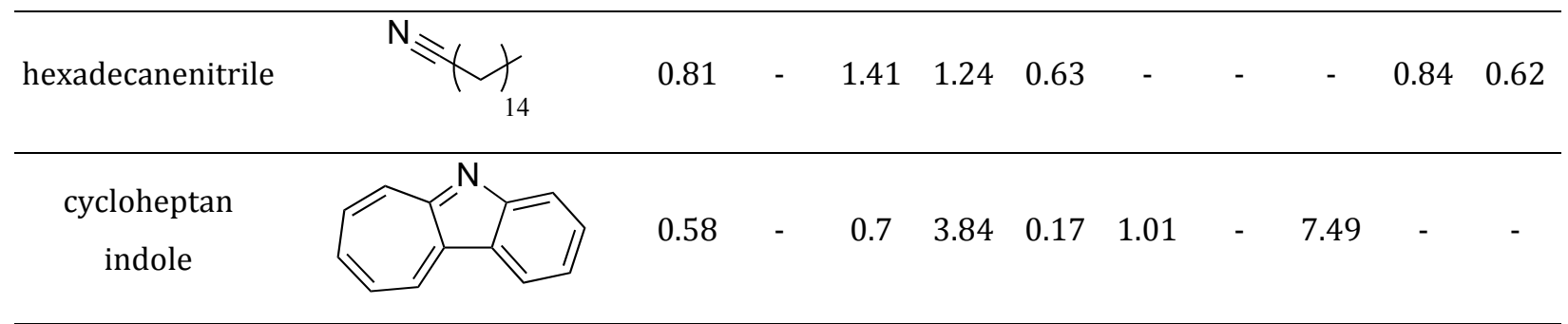

\section{9-hexadecenoic} acid

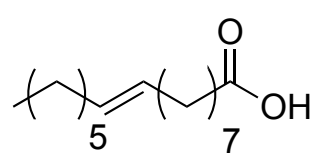

$$
3.630 .32
$$

$0.42 \quad 1.6$

$4.81 \quad 21.07$

\begin{tabular}{llllllllllllll}
\hline cyclic proline & 0.64 & 3.56 & 0.75 & 3.18 & 0.83 & 2.43 & - & - & 1.23 & 7.67 \\
hexadecanoic acid & 3.97 & 30.02 & 3.48 & 12.32 & 2.13 & 7.17 & - & 3.35 & 5.19 & 9.75
\end{tabular}

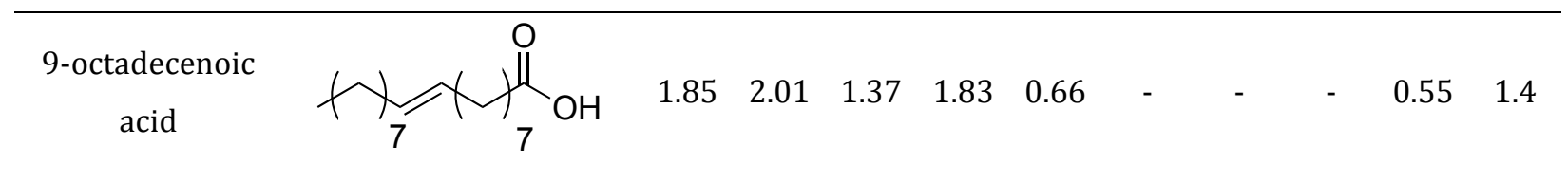

1-Methyl-9H-betacarboline<smiles>Cc1nccc2c1[nH]c1ccccc12</smiles>

0.6 0.27 0.8 $\begin{array}{llllll}0.54 & 1.04 & 0.65 & - & 0.74 & -\end{array}$

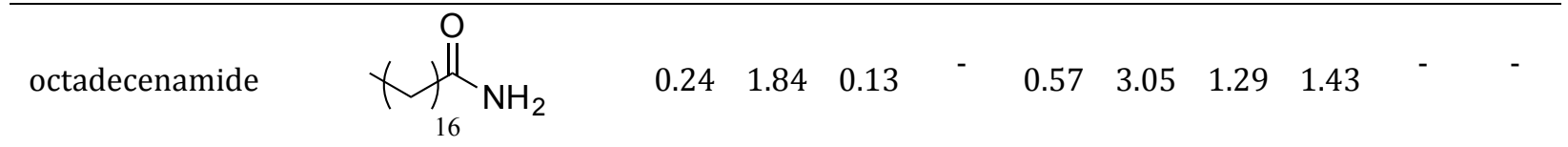

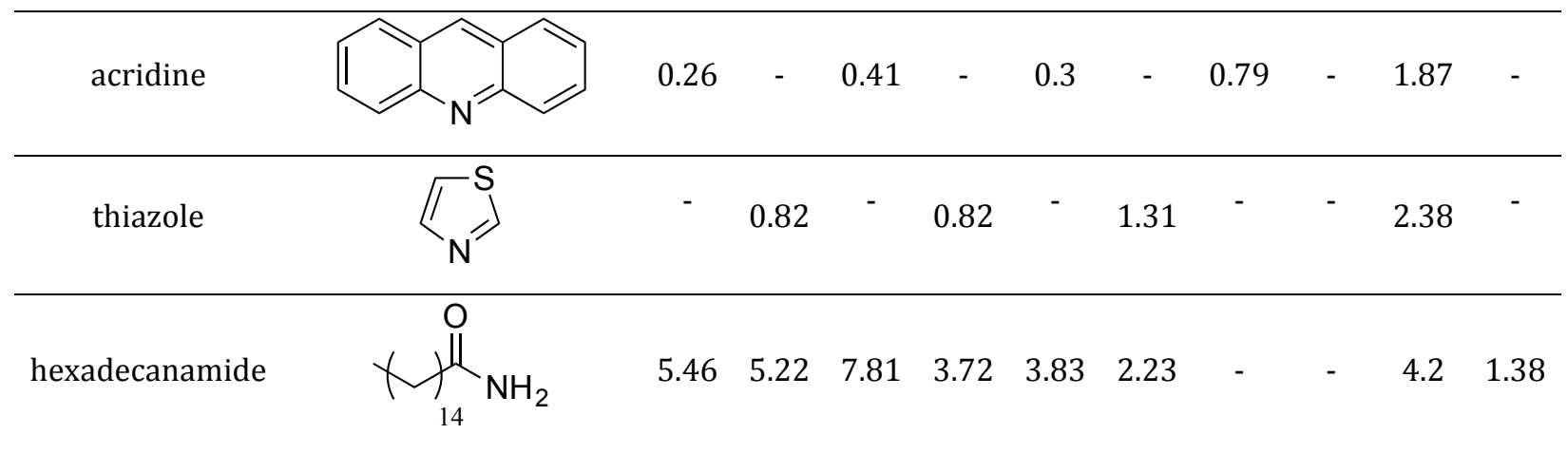




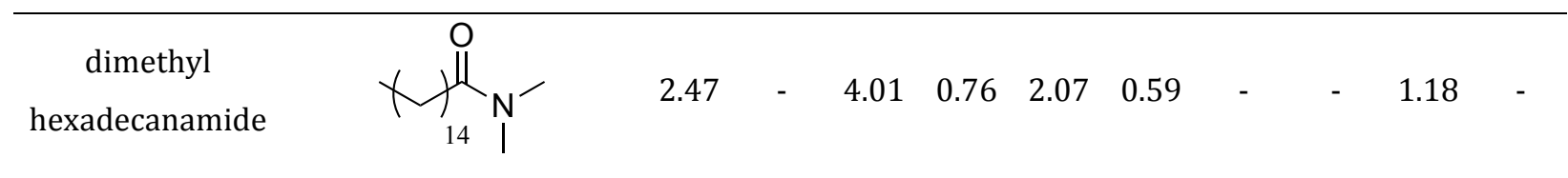

1Not detected

\subsection{Conclusions}

We demonstrated the feasibility of using microbial monocultures as a feedstock for HTL to produce biocrude. The high $\mathrm{N}$ and $\mathrm{O}$ content of the biocrude, for all organisms and all treatment conditions, necessitates additional treatment of the biocrude before its use as a liquid transportation fuel. The cultivation of bacteria with aqueous-phase byproducts from HTL of microalgae [77] provides an opportunity to improve overall utilization of nutrients and total biocrude output in an algal biorefinery.

The E. coli cultivated in the TB (nutrient-rich) media was higher in ash and lower in lipid than the E. coli cultivated in minimal media. The growth media used to cultivate the bacterium did not significantly affect the elemental composition of the harvested biomass. The biocrude yields produced at both isothermal and fast HTL conditions were not significantly different for the two different cultivation media. This insensitivity of the HTL outcomes to the growth media suggest that the aqueous streams that are nutrient-depleted or contain substrates that are not easily metabolized [77] may nevertheless be suitable for biocrude production via $E$. coli cultivation.

The Gram-positive organism, B. subtilis, provided the lowest yield of biocrude compared to all other microorganisms in this study. Its modestly lower lipid content is probably not fully responsible for the reduced yield as the E. coli TB had a similar lipid content, but significantly higher biocrude yields. The B. subtilis biomass did decompose during HTL, however, as the yield of residual solids was only about 4 wt \%. The decomposition products were primarily water-soluble, which might be in part due to this Gram-positive bacterium's cell envelope containing a thick peptidoglycan layer.

S. cerevisiae had a higher average yield of biocrude than did the bacteria. The higher yield also resulted in a higher average recovery of the energy in the biomass. However, $P$. putida had similar yields and is capable of growing on media lacking nutrients and 
containing possible toxins that can inhibit growth, specifically, the by-product aqueousphase from the liquefaction of microalgae [77].

As literature and the results presented herein indicate, fast HTL can, in many cases, lead to higher total biocrude yields than isothermal HTL [113]. The shorter reaction time necessary for fast HTL would reduce reactor size and capital costs in an industrial process. However, in exchange for these benefits, fast HTL biocrudes appear to have a less desirable composition. For example, a higher percentage of the total biocrude exists as the heavy fraction. Fast HTL biocrudes also have higher $\mathrm{O}, \mathrm{N}$, and S content, which is less desirable for use as a biofuel or biofuel precursor compared to isothermal HTL biocrudes. More of the N in the isothermal HTL aqueous phase is converted to $\mathrm{NH}_{3}$, making the $\mathrm{N}$ preferable as a nutrient for algae cultivation [17]. Further economic and environmental analysis of these trade-offs is required to determine which of these processes is preferable for the conversion of biomass to biocrude.

\subsection{Materials and Methods}

This section first describes the cultivation and analysis of the biomass feedstocks and then describes the procedures for the hydrothermal treatment and the collection, separation, and analysis of the product fractions.

\subsubsection{Biomass Cultivation and Harvesting}

We grew all four organisms in various "rich" media containing high concentrations of complex biologically derived materials such as yeast extract and peptone to maximize biomass yield per volume of culture. For the rich media, we used Luria-Bertani medium (10 g/L tryptone, $5 \mathrm{~g} / \mathrm{L}$ yeast extract, $10 \mathrm{~g} / \mathrm{L} \mathrm{NaCl})$ for P. putida, Terrific Broth medium $(12 \mathrm{~g} / \mathrm{L}$ tryptone, $24 \mathrm{~g} / \mathrm{L}$ yeast extract, $4 \mathrm{ml} / \mathrm{L}$ glycerol, $2.31 \mathrm{~g} / \mathrm{L} \mathrm{KH}_{2} \mathrm{PO}_{4}, 12.54 \mathrm{~g} / \mathrm{L} \mathrm{K} \mathrm{KPO}_{4}$ ) for $E$. coli and B. subtilis, and Yeast Peptone Dextrose medium (10 g/L yeast extract, $20 \mathrm{~g} / \mathrm{L}$ peptone, $20 \mathrm{~g} / \mathrm{L}$ dextrose) for S. cerevisiae. We also grew E. coli in a "minimal" medium, which contained only chemically defined substrates and nutrients. For the E. coli minimal medium we used M9 medium (20 g/L glucose, $1 \mathrm{~g} / \mathrm{L} \mathrm{NH}_{4} \mathrm{Cl}, 6 \mathrm{~g} / \mathrm{L} \mathrm{Na} 2 \mathrm{HPO}_{4}, 3 \mathrm{~g} / \mathrm{L} \mathrm{KH}_{2} \mathrm{PO}_{4}$, $0.5 \mathrm{~g} / \mathrm{L} \mathrm{NaCl}, 1 \mathrm{mM} \mathrm{Mg}_{2} \mathrm{SO}_{4}, 0.1 \mathrm{mM} \mathrm{CaCl}$ ). Both seed cultures and final cultures were 
grown in the same media. E. coli grown in Terrific Broth and M9 minimal medium will be referred to as E. coli $\mathrm{TB}$ and E. coli MM, respectively.

To grow the biomass feedstock, we first obtained cryogenically preserved $\left(-80{ }^{\circ} \mathrm{C}\right)$ stocks of Escherichia coli K12 MG1655, Pseudomonas putida KT2440, Bacillus subtilis SB491, and Saccharomyces cerevisiae S288C and grew a $20 \mathrm{~mL}$ seed culture in a $50 \mathrm{~mL}$ test tube. We incubated the seed culture for $24 \mathrm{~h}$ at $30^{\circ} \mathrm{C}$ and agitated it at $250 \mathrm{rpm}$ in a New Brunswick Excella E24 incubator. We then added the seed cultures to fresh growth media at a ratio of $20 \mathrm{~mL}$ seed culture to $2 \mathrm{~L}$ medium in 1.5 to $2.0 \mathrm{~L}$ cultures and grew them for 24 - $48 \mathrm{~h}$ in a New Brunswick I26 or Innova 4400 incubator agitated at $120 \mathrm{rpm}$. Incubation temperatures ranged from $30-37^{\circ} \mathrm{C}$, and growth vessels were capped with a sterile filter to allow for oxygen transport.

We harvested cell cultures by centrifuging them in $1 \mathrm{~L}$ vessels at $5000 \mathrm{rcf}$ (relative centrifugal force) for $15 \mathrm{~min}$ in a Beckman Coulter Avanti J-20XP or Thermo Scientific Sorvall Lynx 6000 centrifuge. To "wash" the biomass to remove potential substrates remaining from the media, we discarded the supernatant media and re-suspended the cell pellets in an equal volume of de-ionized water, then reformed the pellet again with a 20 min centrifugation. We then discarded the supernatant water and re-suspended each cell pellet in $10 \mathrm{~mL}$ of water, transferred this slurry to $50 \mathrm{~mL}$ centrifuge tubes, and centrifuged it once more at $5000-12000 \mathrm{rcf}$ in an Allegra 21R or Eppendorf 5810R centrifuge. We discarded the supernatant water from these tubes and considered the resulting cell pellets raw wet biomass. The collected biomass was always at least a $12 \mathrm{wt} \%$ slurry.

\subsubsection{Biomass Feedstock Analysis}

We dried biomass samples in a $70{ }^{\circ} \mathrm{C}$ oven for 48 hours to completely remove the water. To determine the ash content, we measured $50 \mathrm{mg}$ of the dried biomass into a preweighed aluminum weigh boat. A Ney Vulcan 3-130 muffle furnace heated the sample to $250{ }^{\circ} \mathrm{C}$ from room temperature at a rate of $10^{\circ} \mathrm{C} / \mathrm{min}$. After a $30 \mathrm{~min}$ holding period, the temperature was increased at a rate of $20{ }^{\circ} \mathrm{C} / \mathrm{min}$ to $550{ }^{\circ} \mathrm{C}$ and then held for $30 \mathrm{~h}$. After removing the samples from the furnace, we cooled them in a desiccator to room temperature and then recorded the mass of ash. We sent dried samples of the biomass 
feedstock to Atlantic Microlabs, Inc. for analysis of C, H, N, and S content. We calculated $\mathrm{O}$ content as the difference between $100 \mathrm{wt} \%$ and the combined content of $\mathrm{C}, \mathrm{H}, \mathrm{N}, \mathrm{S}$, and ash.

We developed a lipid analysis method by combining practices from Levine et al. and Lewis et al [123], [124]. and purchased chemicals of > $99 \%$ purity, from Fisher Scientific. We measured approximately $20 \mathrm{mg}$ of dried biomass into a glass test tube and then added $2 \mathrm{~mL}$ of a $5 \mathrm{v} / \mathrm{v} \%$ solution of acetyl chloride in methanol and a magnetic stir bar. We vigorously stirred the reaction mixture ( $>100 \mathrm{rpm}$ ) for $90 \mathrm{~min}$ at $100{ }^{\circ} \mathrm{C}$ using a magnetic stir plate and temperature-controlled heating block. After the holding period, we quenched the reaction by adding $1 \mathrm{~mL}$ of room-temperature de-ionized water. After the solution cooled for $10 \mathrm{~min}$, we added $4 \mathrm{~mL}$ of $\mathrm{n}$-heptane and agitated each tube for $10 \mathrm{~min}$ on a vortexer set to $1000 \mathrm{rpm}$. We centrifuged the mixture for $3 \mathrm{~min}$ at $1500 \mathrm{rcf}$ to separate and then collect the heptane layer for gas chromatographic analysis. We injected $1 \mu \mathrm{L}$ of sample, with a 2:1 split ratio, into an Agilent 7890 gas chromatograph equipped with an Agilent DB-FFAP column ( $30 \mathrm{~m} \times 320 \mu \mathrm{m} \times 0.25 \mu \mathrm{m})$. Helium at a column flow of $1 \mathrm{~mL} / \mathrm{min}$ was the carrier gas. The injector temperature was $250{ }^{\circ} \mathrm{C}$. The oven temperature was maintained at $60^{\circ} \mathrm{C}$ until the injection and then increased to $200{ }^{\circ} \mathrm{C}$ at a rate of $20^{\circ} \mathrm{C} / \mathrm{min}$ and then to $240{ }^{\circ} \mathrm{C}$ at a rate of $5{ }^{\circ} \mathrm{C} / \mathrm{min}$. The final temperature was held for $3 \mathrm{~min}$. We generated calibration curves using a RESTEK Marine Oil mixture of 20 fatty acid methyl esters as an external standard.

We estimated the protein content (wt \%) of the biomass by multiplying its $\mathrm{N}$ content (wt \%) by 6.25 [125], [126]. We calculated the carbohydrate content as the difference between $100 \mathrm{wt} \%$ and the sum of the lipid, protein, and ash contents.

\subsubsection{Hydrothermal Liquefaction}

We constructed Swagelok $®$ reactors using $3 / 8$ " port connectors fitted with a cap on one end and a $3 / 8$ " to $1 / 8$ " union on the other end. The union allowed for the attachment of a 15,000 psi-rated High Pressure Equipment Co. valve, with grafoil packing, for sampling the gas products. The valve was attached via 8.5 " of $1 / 8$ " OD tubing. The nominal volume added by the valve was $0.5 \mathrm{~mL}$. The total volume of the reactor and valve is approximately $2.2 \mathrm{~mL}$ (depicted in Figure 5.5). 


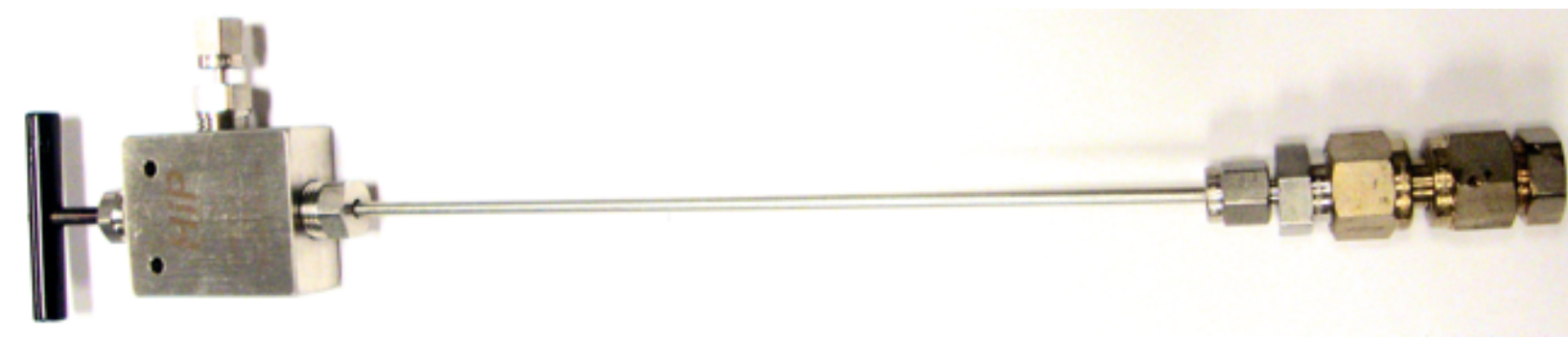

Figure 5.5 Reactor with gas valve assembly

For conventional, isothermal HTL, we loaded $1.35 \mathrm{~g}$ of 12 wt \% biomass slurry to each Swagelok reactor. At this loading, water would fill 95\% of the reactor volume at the reaction conditions. We sealed the reactors and placed them into a Techne Fluidized sand bath set at $350{ }^{\circ} \mathrm{C}$. The reactors were submerged in the sand bath and agitated using a Burrell Wrist Action shaker [11] for $60 \mathrm{~min}$. For fast HTL reactions, done with rapid heating, we loaded the reactor with $0.30 \mathrm{~g}$ of $12 \mathrm{wt} \%$ biomass slurry. This water loading matched previous experiments in our laboratory [113]. After sealing the reactors, they were placed in a $600{ }^{\circ} \mathrm{C}$ sandbath for $1 \mathrm{~min}$. As previously described by Faeth et al., we used dummy reactors (depicted in Figure 5.6) fitted with a thermocouple to record temperature and calculate the heating rate [113]. In both cases, after the desired holding time had elapsed, we removed the reactors from the sandbath and quenched them in a room temperature water bath.

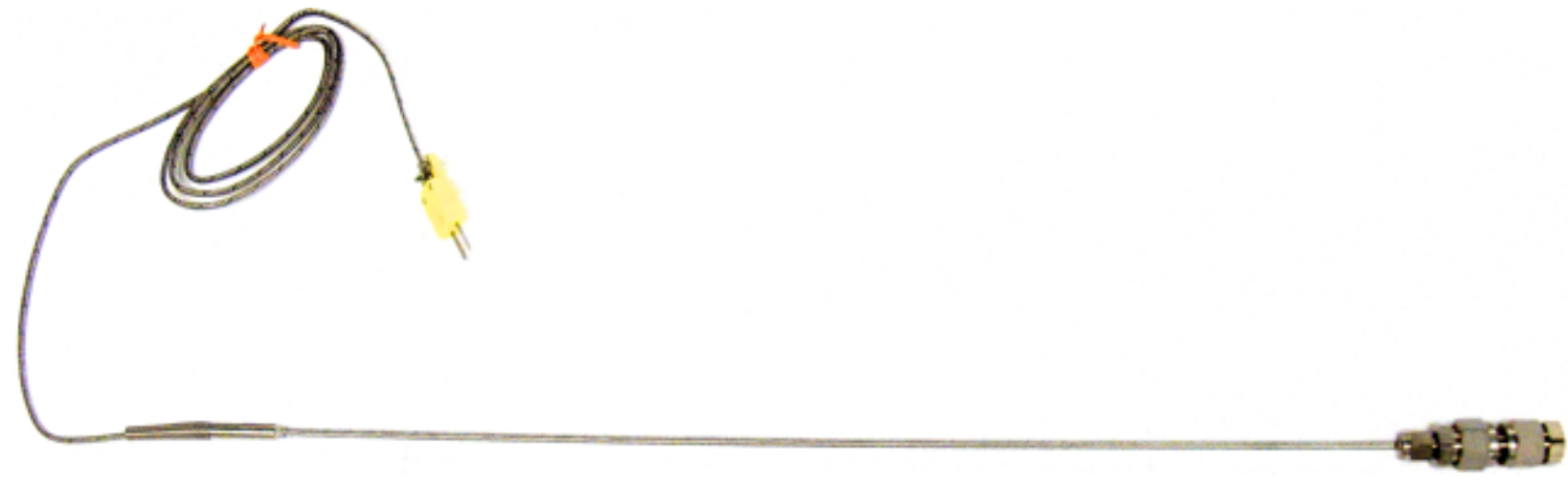

Figure 5.6 Dummy reactor with K-type thermocouple

\subsubsection{Recovery and Analysis of HTL Product Fractions}

We followed the procedure published previously to collect and separate the solids, aqueous-phase products, light biocrude (hexane-solubles), heavy biocrude (hexaneinsoluble, dichloromethane soluble), and gas product fractions from each reactor [11], [113]. We report the gravimetric yield of each product fraction as its mass divided by the 
mass of biomass loaded into the reactor on a dry basis (wt \%). We also report the biocrude yields on a dry, ash-free basis (wt \% daf) as the ash content cannot contribute to the yield of biocrude.

Solvent-free samples of the light and heavy biocrude were sent to Atlantic Microlab, Inc. for measurement of wt \% $\mathrm{C}, \mathrm{H}, \mathrm{N}$, and $\mathrm{S}$. $\mathrm{O}$ content in the light and heavy biocrudes was calculated by difference. We report elemental distribution as the mass of an element in each of the product fractions per total mass of that element in the biomass. We diluted the aqueous phase 1:600 with deionized water and measured $\mathrm{NH}_{3}$ in the aqueous-phase using methods described previously [11].

The hexane-soluble product, also referred to as the light biocrude, was analyzed with an Agilent 6890N GC fitted with an Agilent 5973 MS. We injected $1 \mu \mathrm{L}$ into a $300{ }^{\circ} \mathrm{C}$ split injection port using a split ratio of 2:1 onto an Agilent HP-5 capillary column (50 m $\times$ $200 \mu \mathrm{m} \times 0.33 \mu \mathrm{m})$. The oven was set to $100^{\circ} \mathrm{C}$ and the temperature increased to $300^{\circ} \mathrm{C}$ at a rate of $5{ }^{\circ} \mathrm{C} / \mathrm{min}$ immediately after injection. The samples exited the column into an electron ionization mass spectrometer. We used matching software to tentatively identify molecular constituents in the biocrude sample based on mass spectra.

We calculated the higher heating value of the light and heavy biocrude using the Boie formula [127] and the elemental composition data (wt \%) on a dry basis:

Higher Heating Value $(\mathrm{MJ} / \mathrm{kg})=0.3516 \cdot \mathrm{C}+1.16225 \cdot \mathrm{H}+0.0628 \cdot \mathrm{N}+0.10465 \cdot \mathrm{S}-0.1109 \cdot 0$

We used the heating values and gravimetric yields of the biocrude product fractions to estimate the energy recovered in the biocrudes from the original biomass. We report the average of at least three replicate experiments, unless otherwise noted. When available, we report experimental error as one standard deviation.

\subsubsection{Control Experiments}

We added approximately $160 \mathrm{mg}$ of dried biomass to a glass test tube, which is similar to the biomass loading in the reactor for isothermal liquefaction. We then added 9 $\mathrm{mL}$ of dichloromethane and agitated the samples on a vortexer set at $1000 \mathrm{rpm}$ for $1 \mathrm{~h}$. After mixing, we added $1.2 \mathrm{~mL}$ of deionized water, which mimics the water loading in a reactor, and agitated the samples for another hour. We then followed the work-up 
procedure described previously to collect and measure the yield of each of the product fractions, except for gas [11]. This control experiment provides the yields of each product fraction available from the biomass simply by wet extraction without HTL. 


\section{Chapter 6}

\section{Growth of Algae on AqAl Pre-Processed by Bacterial Culture}

\subsection{Summary}

We grew alga Chlorella vulgaris in media containing various concentrations of the aqueous phase product (AqAl) generated from hydrothermal liquefaction of Nannochloropsis oculata algae biomass as its sole carbon and nitrogen source. We used both raw AqAl directly from the hydrothermal reaction as well as AqAl that had first been pre-processed in two-day bacterial growth cultures. The bacteria used were Escherichia coli, Pseudomonas putida, and a strain of E. coli evolved to better utilize AqAl.

Growth of Chlorella in 0.5 vol\%, 3 vol\%, and 6 vol\% AqAl (using both raw and preprocessed AqAl) was monitored over two weeks. Bacterial pre-processing had a positive effect on the growth of algae at the highest concentration of AqAl.

\subsection{Introduction}

The prospect of biomass conversion into biofuels presents concerns in regards to nutrient and fertilizer recycle. Regardless of biomass source or desired fuel, the final product will be a hydrocarbon stripped of most of the nitrogen and phosphorus present in the initial biomass. If discarded in wastewater, these compounds must be handled at a wastewater processing operation, and nitrogen and phosphorus, along with other nutrients, must also be purchased to grow more biomass, imposing a double charge to the biofuel process. In one proposed process, the production of "biocrude" oil from algal biomass, the internal recycling of nitrogen and phosphorus is key to the financial feasibility and overall sustainability of the system [67], [79].

Microalgae have the potential to be grown year-round in much larger yields than terrestrial biomass and without competition for arable farmland or even fresh water [128]. Wet algae biomass slurries can be processed via hydrothermal liquefaction (heated to subcritical temperatures and pressurized), creating a biocrude oil similar to crude petroleum. But the aqueous byproduct formed from this algae reaction (AqAl) contains the majority of 
the nitrogen and phosphorus from the initial biomass [11]. Initial research into refeeding the AqAl back to algae growth was met with limited success, showing that growth is possible, but there is also an inhibition effect [13], [14]. More recent studies have shown better algae regrowth on AqAl with micronutrient supplementation [17], but algae still has problems dealing with organic nitrogen.

In this study, we propose bacterial preculture as a way to make AqAl more suitable for algal regrowth. In addition to nitrogen and phosphorus compounds, AqAl also contains a significant fraction of the organic carbon present in the initial biomass, and the bacterial strains Escherichia coli and Pseudomonas putida have been shown to grow using AqAl as the sole $\mathrm{C} / \mathrm{N} / \mathrm{P}$ source and tolerate AqAl concentrations much higher than what algae are capable of [77]. Bacterial biomass has been shown to be a feasible source of supplemental biomass for biocrude production via hydrothermal liquefaction [78], which could increase efficiency and reduce overall cost per volume of oil at a consolidated algae refinery system [79]. In nature, bacteria and microalgae have a symbiotic relationship where bacteria utilize dead algae as a substrate for growth, produce vitamins such as cobalamin (B12), and also remineralize the organic nitrogen and phosphorus compounds in the debris, eventually used by the algae [49], [129].

\subsection{Overview}

An outline of this project is presented in Figure 6.1. We generated AqAl from the hydrothermal liquefaction of Nannochloropsis oculata, an alga that can be readily purchased in substantial quantities. We then made simple bacterial growth media (SBM) using the AqAl as the sole source of C/N/P and grew E. coli K12 MG1655, P. putida KT2440, and E. coli $\mathrm{K} 2 \mathrm{C} 2$ (an evolved strain derived from K12 which shows enhanced metabolism of AqAl) and grew 2-day cultures of each. Cells were then filtered from the cultures and the filtrate was added to an algal growth media with no $\mathrm{N}$ or P source (Stripped COMBO media). We compared algae growth on such media to growth on media made using bacterial media that had not been used to culture bacteria. Chlorella vulgaris was used as the regrowth algae. 


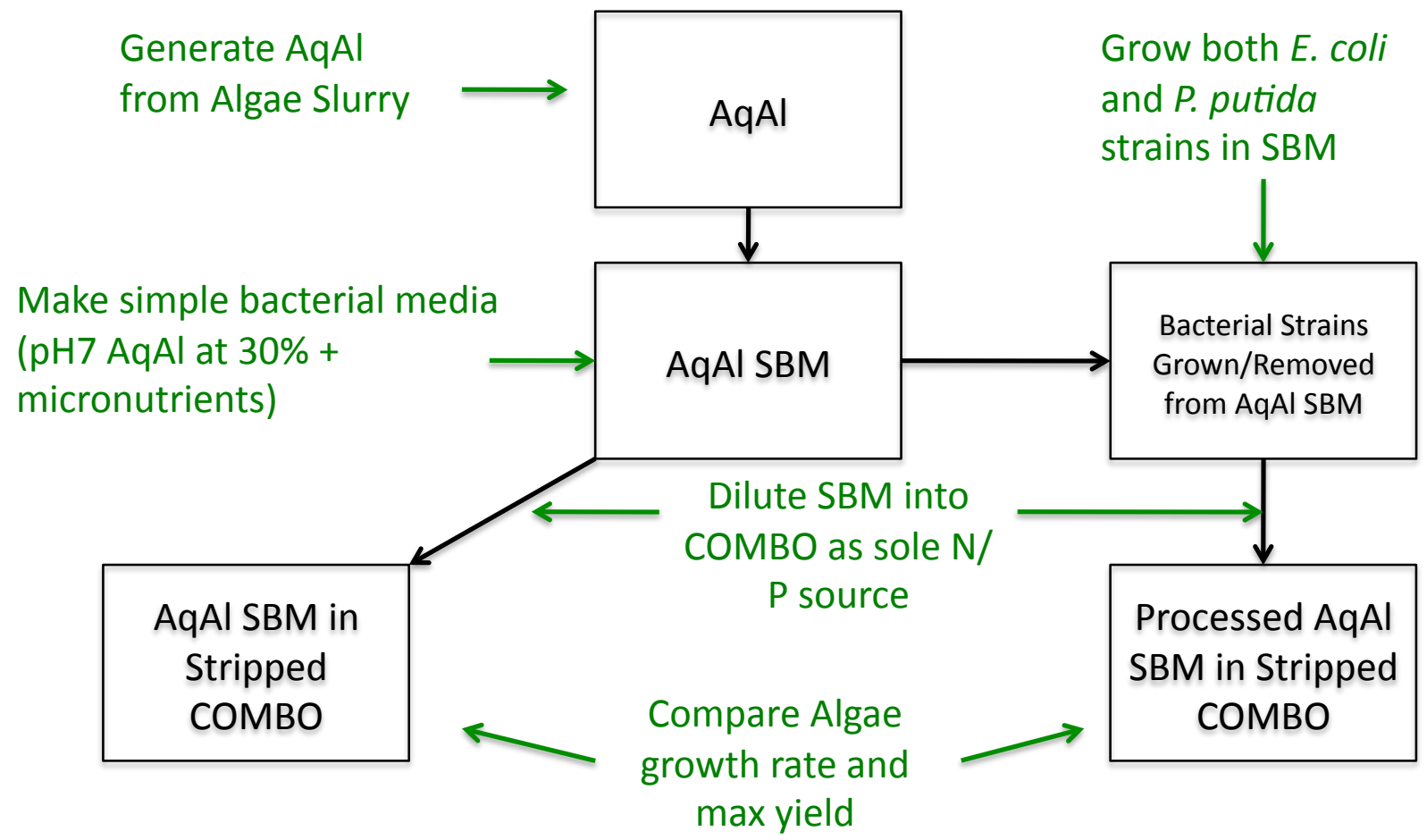

Figure 6.1 Plan for investigating the effects of bacterial preculture on algae regrowth

\subsection{Results and Discussion}

We grew the bacterial strains in simple bacteria medium (SBM) using AqAl as the sole $\mathrm{C} / \mathrm{N} / \mathrm{P}$ source for two days, filtered out the cells, then used this "processed AqAl" as the sole N/P source in various strengths of algae growth media (COMBO media). We also made COMBO media with SBM that had not been used to grow bacterial cultures (unprocessed AqAl). We grew algae strain C. vulgaris in various strengths of unprocessed AqAl media and monitored growth over two weeks (Figure 6.2). We saw a decrease in total growth over 13 days across all concentrations of AqAl. Between $0.5 \%$ and 2\% AqAl, cultures had decreasing levels of growth on the 6-day midpoint yet a similar total growth over 13 days. From $2 \%$ to $6 \% \mathrm{AqAl}$, there was a steadily decreasing level of growth at all time points, with apparent stabilization between $6 \%$ and $7 \%$. We used these data to determine a baseline inhibition level of AqAl media and chose $0.5 \%, 3 \%$, and $6 \%$ as concentrations representing mild, moderate, and severe growth inhibition. 
We then made COMBO media with AqAl SBM processed by E. coli K12, E. coli K2C2, and $P$. putia, as well as unprocessed AqAl SBM, at concentrations of $0.5 \%, 3 \%$, and $6 \% \mathrm{AqAl}$. We grew C. vulgaris microplate cultures in these media over the course of 18 days, measuring fluorescence every 2-4 days Figure 6.3. There was similar growth between the unprocessed $\mathrm{AqAl}$ and $\mathrm{K} 2 \mathrm{C} 2$ processed $\mathrm{AqAl} 0.5$ vol\% media, with the $\mathrm{K} 12$ and P. putida media showing a lag phase followed by growth to similar final fluorescence after 18 days. At 3 vol\% AqAl, all media produced very similar growth results, with unprocessed AqAl medium showing a slightly longer lag phase evident between 9 and 11 days. At 6 vol\% $\mathrm{AqAl}$, all processed media showed better growth, with unprocessed AqAl medium showing a longer lag phase, but eventual growth to a similar final fluorescence. 


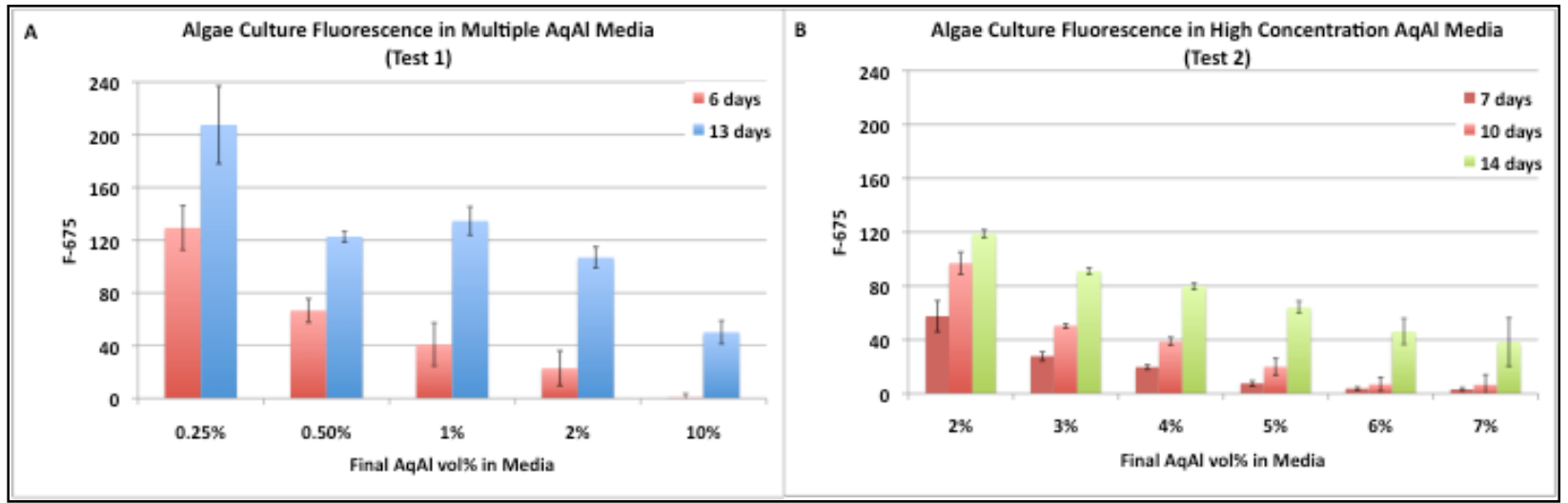

Figure 6.2 Fluorescence measurements of algae cultures grown in unprocessed AqAl COMBO media in two microplate studies: Wide range (A) and High Concentration (B). Error bars represent the standard deviation of three replicates.
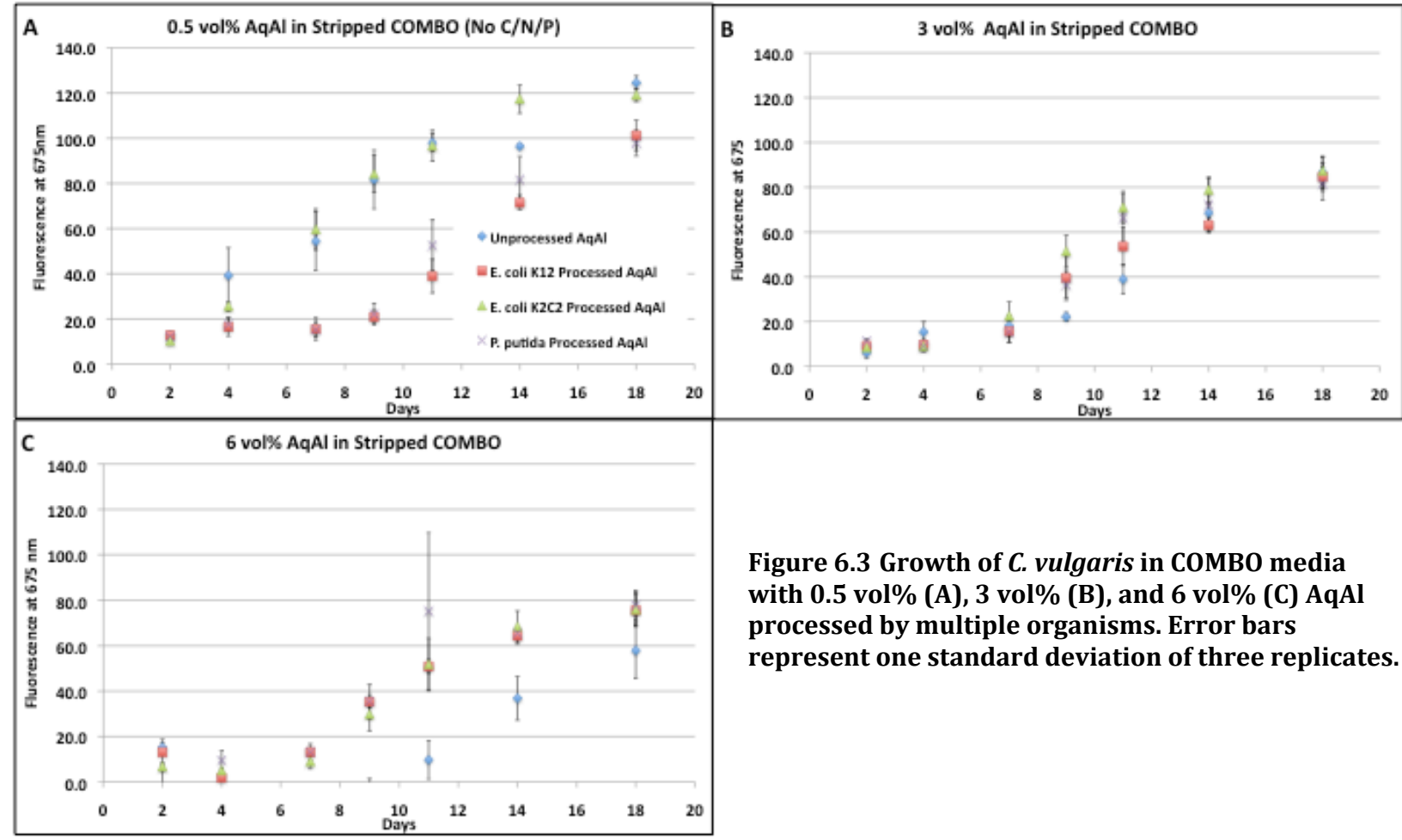

Figure 6.3 Growth of $C$. vulgaris in COMBO media with 0.5 vol\% (A), 3 vol\% (B), and 6 vol\% (C) AqAl processed by multiple organisms. Error bars represent one standard deviation of three replicates. 


\subsection{Conclusions}

These preliminary results show that an intermediate culture of bacteria on the aqueous phase product from hydrothermal liquefaction of algae biomass has the potential to make a positive impact on growth performance when the product is eventually refed to algae. There is a slight negative impact on growth (depending on the strain used for preprocessing) at a low concentration of AqAl in the algae medium. This may be due to the preculture bacteria metabolizing some limiting nutrient of the algae, or consuming an organic carbon substrate, such as acetate or glycerol, that $C$. vulgaris is capable of using for mixotrophic growth. However, at a high level of AqAl, all preprocessed media show better growth results than unprocessed AqAl medium. At this point, the toxic compounds present in the AqAl may be causing a stronger negative effect on the algae culture than the positive effect caused by the nutrients it provides. Bacterial preculture may have served to either metabolize these compounds, convert them into something less harmful, or simply sequestered them within bacterial biomass.

Further work could be done to analyze carbon, nitrogen, and phosphorus compounds in AqAl after bacterial culture, then after algae culture in order to look for selective utilization. There should also be study of algae consumption of AqAl generated by its own species' biomass, and several rounds of reuse (to simulate a recycle stream in a continuous process), both with and without bacterial pretreatment.

\subsection{Methods and Materials}

E. coli and $P$. putida were grown in simple bacterial growth medium containing only micronutrients and 30 vol\% aqueous phase product (AqAl) from a Nannochloropsis oculata algae biomass hydrothermal reaction. Cells were filtered from the cultures and the remaining filtrate was mixed at various concentrations with algae minimal media as the sole nitrogen and phosphorus source. Algae strain Chlorella vulgaris was grown in these media and its growth progress monitored over time.

\subsubsection{Strain Selection}

Nannochloropsis oculata was supplied from Reed Mariculture Inc. as the source of algae biomass. A slurry of $20 \mathrm{wt} \%$ solids was used to generate AqAl in a manner identical to 
that described in Sections 3.6.1. Bacterial strains Escherichia coli K12 MG1655 and Pseudomonas putida KT2440, along with E. coli K2C2, an evolved strain derived from E. coli K12, were grown in media made from the AqAl. Algae strain Chlorella vulgaris was grown on algae media made with post-culture bacterial media as the N/P source. $C$. vulgaris stocks in the form of live cultures on solid agar medium were supplied by the Bradley Cardinale Lab Group of the University of Michigan School of Natural Resources.

\subsubsection{Bacterial Culture}

We made a simple bacteria media (SBM), containing a micronutrient mix of $2.91 \mathrm{nM}$ $\left(\mathrm{NH}_{4}\right)_{6} \mathrm{Mo}_{7} \mathrm{O}_{2}, 401.1 \mathrm{nM} \mathrm{H}_{3} \mathrm{BO}_{3}, 30.3 \mathrm{nM} \mathrm{CoCl} 2\left(\mathrm{H}_{2} \mathrm{O}\right)_{6}, 9.61 \mathrm{nM} \mathrm{CuSO}{ }_{4}\left(\mathrm{H}_{2} \mathrm{O}\right)_{5}, 51.4 \mathrm{nM} \mathrm{MnCl}$, 6.1nM ZnSO 4 H2O, 0.01mM FeSO $4\left(\mathrm{H}_{2} \mathrm{O}\right)_{7}, 1 \mathrm{mM} \mathrm{Mg}_{2} \mathrm{SO}_{4}$, and $0.1 \mathrm{mM} \mathrm{CaCl}_{2}$, sterilized with a Corning $0.2 \mu \mathrm{m}$ vacuum filtration system. It also contained $30 \mathrm{vol} \% \mathrm{AqAl}$ (neutralized to $\mathrm{pH}$ 7 with $\mathrm{HCl}$ ) as the sole $\mathrm{C} / \mathrm{N} / \mathrm{P}$ source.

Cryostocks (stored at $-80^{\circ} \mathrm{C}$ ) of E. coli K12, E. coli K2C2, and P. putida were added to $2 \mathrm{~mL}$ of SBM in capped $10 \mathrm{~mL}$ Falcon-brand sterile plastic tubes and incubated overnight at $30{ }^{\circ} \mathrm{C}$ in a New Brunswick E24 incubator agitated at $250 \mathrm{rpm}$. One hundred $\mu \mathrm{L}$ of each overnight culture were then added to $10 \mathrm{~mL}$ of SBM in capped $50 \mathrm{~mL}$ Falcon tubes and incubated under the same conditions for 48 hours. A separate sample of $10 \mathrm{~mL}$ of SBM with no cells added was also incubated in this fashion. After 48 hours, the three cell cultures and one blank media tube were individually filtered with a Millipore-brand $0.22 \mu \mathrm{m}$ Steriflip filtration system. The filtrate samples from cell cultures were considered "processed AqAl SBM" and the filtrate from the blank media sample was considered "unprocessed AqAl SBM".

\subsubsection{Algae Culture}

Algae growth medium was based on Modified COMBO Medium formulated by the University of Texas Culture Collection of Algae (http://web.biosci.utexas.edu/utex/mediaDetail.aspx?mediaID=162). "Stripped COMBO" consisted of a micronutrient base of $2.5 \mathrm{mM} \mathrm{CaCl}_{2}, 1.5 \mathrm{mM} \mathrm{MgSO}_{4}, 1 \mathrm{mM} \mathrm{Na}_{2} \mathrm{SiO}_{3}, 3.9 \mathrm{mM}$ $\mathrm{H}_{3} \mathrm{BO}_{3}, 1 \mathrm{mM} \mathrm{KCl}, 110 \mu \mathrm{M} \mathrm{Na} 2 \mathrm{EDTA}, 40 \mu \mathrm{M} \mathrm{FeCl}_{3}, 0.04 \mu \mathrm{M} \mathrm{CuSO}_{4}, 0.77 \mu \mathrm{M} \mathrm{ZnSO}_{4}, 0.5 \mu \mathrm{M}$ $\mathrm{CoCl}_{2}, 9.1 \mu \mathrm{M} \mathrm{MnCl}, 0.99 \mu \mathrm{M} \mathrm{Na} \mathrm{MoO}_{4}, 0.077 \mu \mathrm{M} \mathrm{H}_{2} \mathrm{SeO}_{3}, 0.11 \mu \mathrm{M} \mathrm{Na}_{3} \mathrm{VO}_{4}, 0.1 \mu \mathrm{M}$ cyanocobalamin (vitamin B12), $0.1 \mu \mathrm{M}$ Biotin, $1 \mu \mathrm{M}$ thiamine. Various concentrations of 30 
vol\% AqAl SBM were added to Stripped COMBO as the N/P source. The finished media were titled according to the final concentration of pure AqAl in the media. For instance, a " 3 vol\% AqAl COMBO medium" consisted of 90 vol\% Stripped COMBO and 10 vol\% SBM, since the SBM contained 30 vol\% AqAl to begin with.

C. vulgaris was grown for one week in $50 \mathrm{ml}$ of $0.5 \%$ AqAl COMBO medium in a $250 \mathrm{~mL}$ flask with a sterile-filter top, under direct fluorescent tube lighting at $20{ }^{\circ} \mathrm{C}$ and an agitation of $150 \mathrm{rpm}$. A $20 \mathrm{~mL}$ culture sample was then centrifuged at $12000 \mathrm{rcf}$ and resuspended in $1 \mathrm{~mL}$ of sterile DI water to make a concentrated algae inoculum suspension. The optical density at $675 \mathrm{~nm}$ was measured and inoculum was added to individual $800 \mu \mathrm{L}$ cultures of various media such that their starting optical density was $\sim 0.02$. These $800 \mu \mathrm{L}$ cultures were grown simultaneously in Corning-brand 48-well microplates in the same light-incubation conditions as the initial inoculum culture. The microplates were occasionally removed from the light-incubator and analyzed with a Spectramax M5 microplate reader. The fluorescence of each culture well at $675 \mathrm{~nm}$ (485nm excitation) was measured. This is a common technique for measuring algae chlorophyll content [130], and is an indirect indicator or algae biomass concentration. Over time, some flocculation and settling (or perhaps precipitate formation) was noticed, so cultures were individually agitated with sterile micropipette tips immediately before fluorescence ("F675") measurement to ensure homogeneity. In all growth tests, wells of media with no algae added were included for each concentration of AqAl used and their F675 was subtracted from the F675 of culture wells to account for native fluorescence of AqAl.

Initial tests to determine general AqAl growth inhibition on algae were done with $0.25 \mathrm{vol} \%$ to $10 \mathrm{vol} \%$ (unprocessed) AqAl COMBO media with fluorescence measurements taken at 6 and 13 days. These showed some growth inhibition between $0.25 \%$ and $0.5 \%$ and severe inhibition between $2 \%$ and $10 \%$. A second test was carried out in single percent increments between $2 \%$ and $7 \%$ to further investigate the growth inhibition in this range.

We then performed a growth test using AqAl COMBO made from each processed AqAl SBM (the filtrate after E. coli $\mathrm{K} 12$, E. coli $\mathrm{K} 2 \mathrm{C} 2$, and P. putida) as well as unprocessed AqAl SBM. We used 0.5 vol\%, 3 vol\%, and 6 vol\% AqAl COMBO in the final to represent 
minimal, moderate, and severe inhibition noticeable within two weeks, as determined by the initial growth tests. F675 measurements were taken every 2-4 days for 18 days. 


\section{Chapter 7}

\section{Concluding Remarks and Future Directions}

\subsection{Concluding Remarks}

In this work we have investigated several unit operations in a theoretical algae biofuel process and ways to improve upon them. We studied the products formed from hydrothermal liquefaction of algae biomass, the culture of microbes on the aqueous product, the improvement of these microbes' utilization of this product via adaptive evolution, the hydrothermal liquefaction of microbial biomass, and finally, the reefed of aqueous phase to algae growth operations after pre-culturing bacteria with it.

Data from several of these studies has been used in a life cycle analysis by Orfield et al [79] that shows a bacterial culture on AqAl may indeed be beneficial to the overall sustainability of an algae HTL process when compared to an alternate strategy of using hydrothermal gasification to convert carbon in AqAl to methane (Figure 7.1). Once carbon dioxide has been fixed to algae biomass in a slow and expensive algae pond operation (requiring large volumes and surface areas for light), only a fraction of the carbon is initially converted into biocrude oil. If the leftover aqueous carbon is completely bioavailable for mixotrophic algae growth, a microbial culture may still be beneficial to the system due to the ability of bacteria to grow faster and to higher densities in enclosed tanks. So even if AqAl bioavailability and toxicity to algae were not issues (which further research indicates they most certainly are), bacteria still could reduce a consolidated algae biorefinery volume and land area and, therefore, reduce cost per gallon of biocrude. However, this comes at the cost of having more energy spent and more overall $\mathrm{CO}_{2}$ produced per volume of oil in comparison to the hydrothermal gasification approach, which generates savings in these areas from burning the methane produced for electricity (Figure 7.2). 
A) CHG Pathway

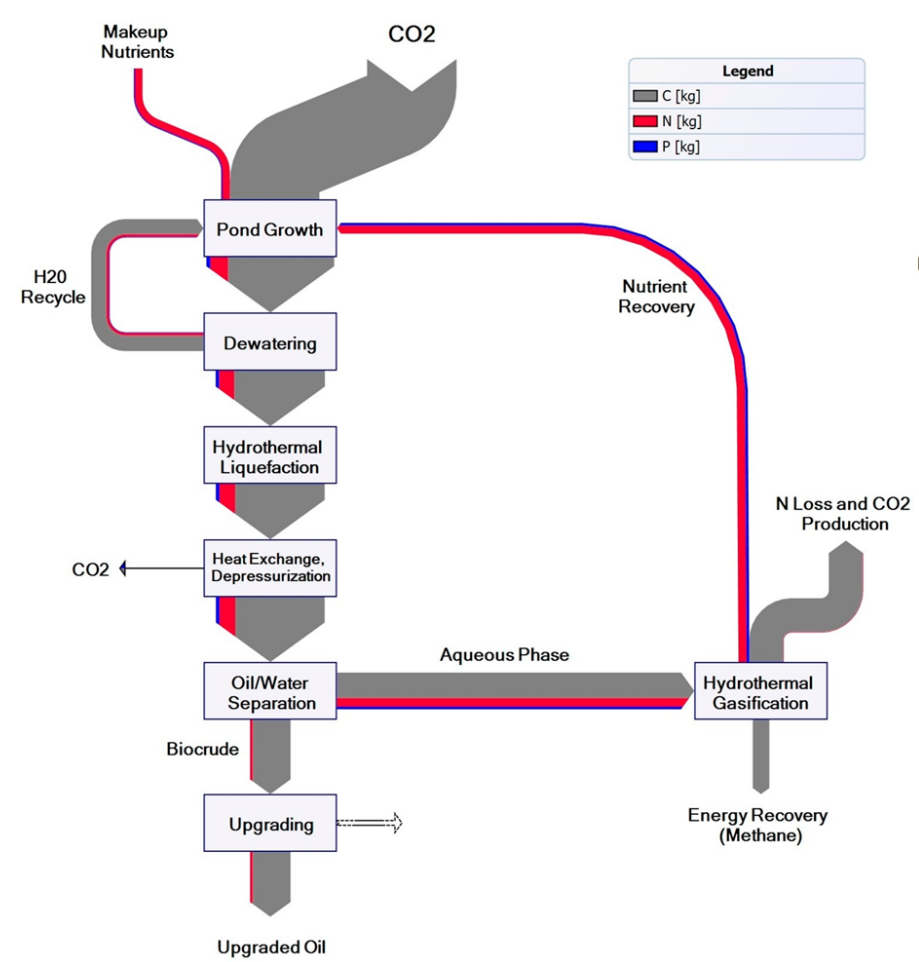

B) Regrowth Pathway

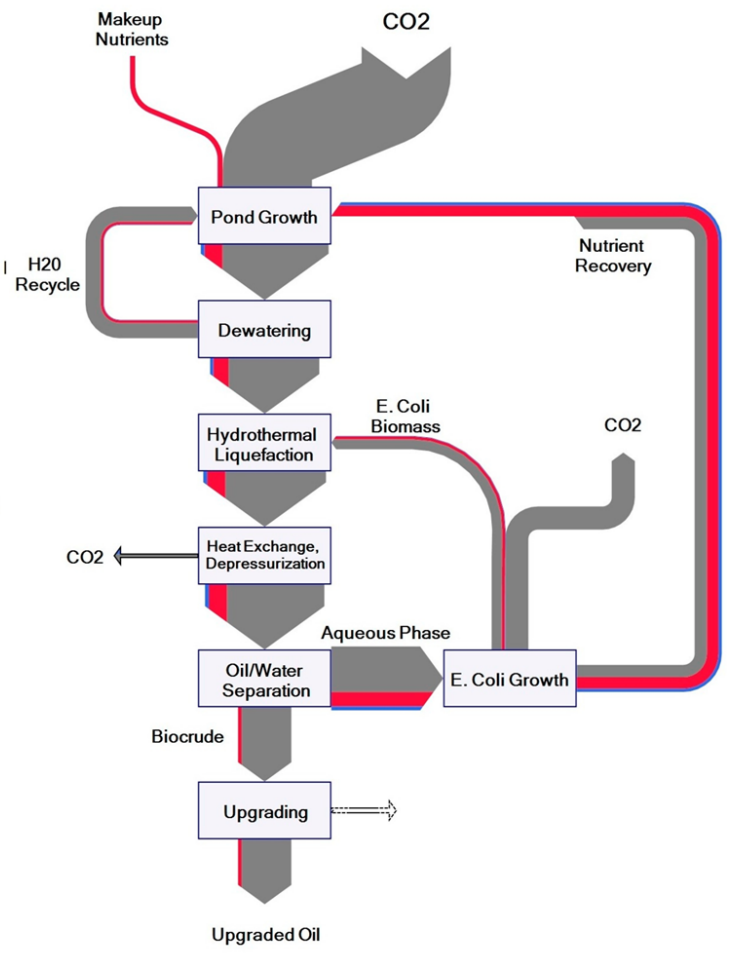

Figure 7.1 Process plow charts for the two theoretical processes analyzed by Nolan et al. "A" contains a hydrothermal gasification step for AqAl to produce methane for energy recovery purposes, while " $B$ " contains an E. coli growth operation to produce supplemental biomass for the hydrothermal liquefaction. The figure is adapted from [79]. 


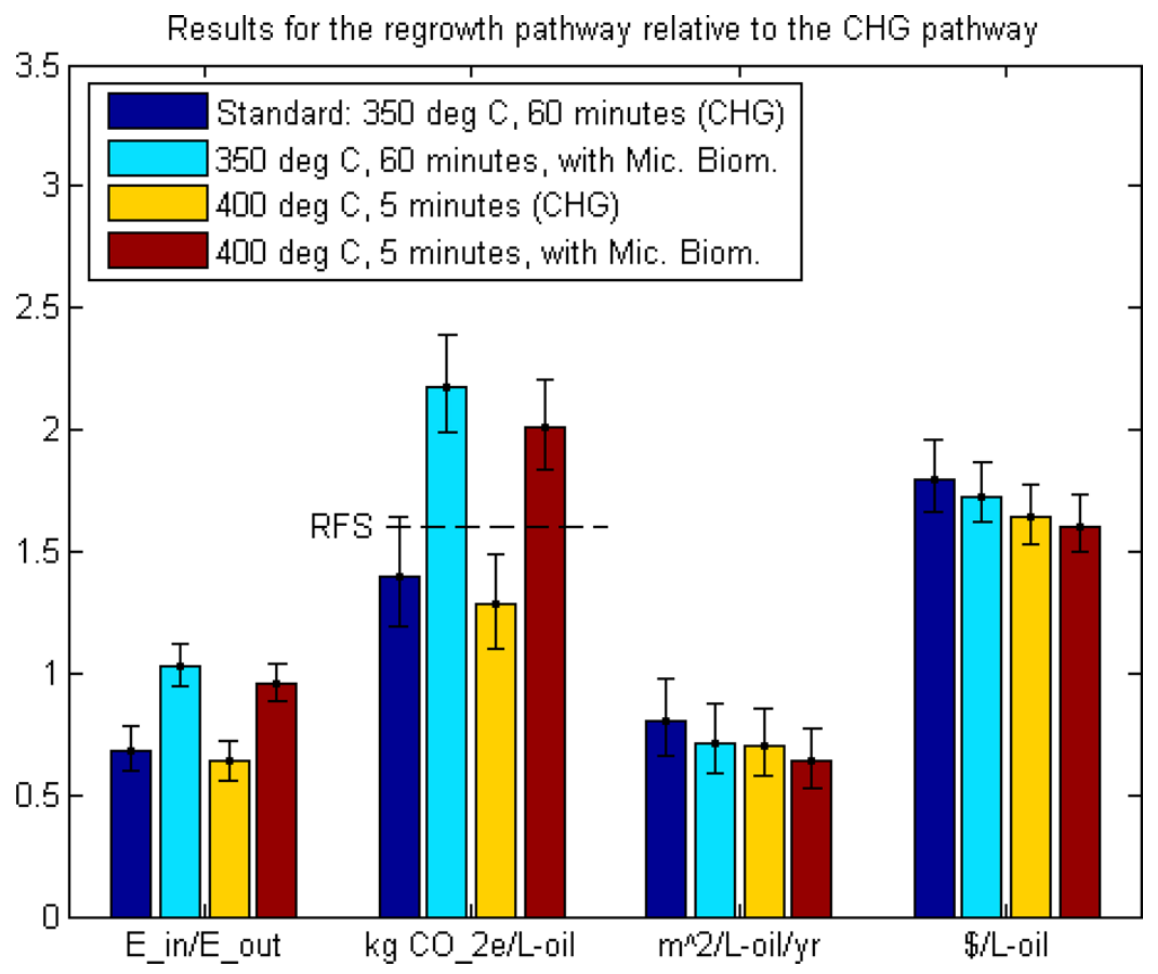

Figure 7.2 Results from Nolan et al showing four process metrics for two HTL conditions (350 ${ }^{\circ} \mathrm{C}$ for 60 minutes and $400{ }^{\circ} \mathrm{C}$ for 5 minutes) with both hydrothermal gasification (CHG) and microbial biomass regrowth approaches to AqAl processing. Properties measured are (1) energy used in the process per energy produced in the form of biocrude, (2) mass of $\mathrm{CO}_{2}$ emitted per liter of oil produced (dotted line is the Renewable Fuel Standards advanced biofuel classification limit), (3) land area of process footprint needed per liter of oil produced per year, and (4) price per liter of oil produced. Figure is adapted from [79]. 


\subsection{Future Directions}

\subsubsection{Hydrothermal Reaction Technology}

At the beginning of this project, in 2009, hydrothermal reaction was still competing with low-temperature carbonization, high-temperature gasification, and lipid extraction as the ideal process for conversion of algae biomass to fuels. While there is still no complete consensus among the research community for which one is the best, HTL is in the lead. Future focus on HTL will need to shift from simply the generation of large biocrude yields to the upgrading of biocude into a final drop-in petroleum replacement. There is also significant scale-up work to be done, from small batch reactors to large continuous operations.

\subsubsection{AqAl Characterization}

Characterization of biocrude oil, the intended product of HTL, initially took precedence over AqAl characterization, but this has caught up in the past 3-4 years due to the increasingly apparent challenge of nutrient recycling. General elemental fractioning data [19] have been followed by long lists of organic compounds [16], but the next challenge is to pinpoint the specific components that present challenges toward algal or microbial culture. We have made some initial steps into investigating this in this dissertation.

\subsubsection{Next Generation Sequencing Technology}

Full genome and RNA transcriptome sequencing was crucial in analyzing the strains generated from the evolution experiment in Part III. Evolution and resequencing like this has been performed with chemically-defined growth media containing known selection factors [42], [80], but this is the first project to our knowledge that goes into such genetic detail after evolution with a mixture of unknown selection factors. Inspection of acquired mutations not only indicated adaptations the bacteria strains had made to their challenging environment, but also provided insight on what those challenges were in the first place.

The availability, speed, and affordability of full genome sequencing is continuing to improve at an exceptional rate [43], and methods to analyze the vast amounts of data generated are also advancing. This study is an example of how this technology has 
advanced to the point where it can be used as an analytical tool by scientists that are not necessarily specialists in bioinformatics. We expect this trend to continue and for sequencing technology to become more widely available and useful to an increasingly diverse set of professions.

Our own data sets from genomic and transcriptomic sequencing runs could be investigated further. We could analyze small insertions and deletions in the genome of evolved E. coli strains instead of just single nucleotide polymorphisms. We could also use transcriptomic data to generate new specific media conditions (acid stress, amino acid supplementation, etc.) to grow evolved strains in for further elucidation of specific challenges involved with AqAl.

\subsubsection{Algae Culture on AqAl}

The properties of AqAl for use in algae culture after pre-treatment by bacteria were only briefly investigated in this project and not to the extent of other groups that had investigated recycling of raw AqAl. Other studies should be carried out to fully understand the implications of bacterial pre-treatment, including elemental analysis of AqAl before and after both bacterial and algal growth, growth of algae on AqAl generated from its own species' biomass, and multiple cycles of algae growth / HTL / bacterial growth / AqAl recycle to simulate a continuous algae refinery process. 


\section{References}

[1] H. C. Greenwell, L. M. Laurens, R. J. Shields, R. W. Lovitt, and K. J. Flynn, "Placing microalgae on the biofuels priority list: a review of the technological challenges," J. R. Soc. Interface, vol. 7, pp. 703-726, 2009.

[2] L. Brennan and P. Owende, "Biofuels from microalgae-A review of technologies for production, processing, and extractions of biofuels and co-products," Renew. Sustain. Energy Rev., vol. 14, no. 2, pp. 557-577, Feb. 2010.

[3] S. Haghighi Mood, A. Hossein Golfeshan, M. Tabatabaei, G. Salehi Jouzani, G. H. Najafi, M. Gholami, and M. Ardjmand, "Lignocellulosic biomass to bioethanol, a comprehensive review with a focus on pretreatment," Renew. Sustain. Energy Rev., vol. 27, pp. 77-93, Nov. 2013.

[4] A. F. Clarens, E. P. Resurreccion, M. A. White, and L. M. Colosi, "Environmental life cycle comparison of algae to other bioenergy feedstocks," Environ. Sci. Technol., vol. 44, pp. 1813-1819, 2009.

[5] N. D. Orfield, A. J. Fang, P. J. Valdez, M. C. Nelson, P. E. Savage, X. N. Lin, and G. A. Keoleian, "Life Cycle Design of an Algal Biore fi nery Featuring Hydrothermal Liquefaction: E ff ect of Reaction Conditions and an Alternative Pathway Including Microbial Regrowth,” 2014.

[6] E. R. Venteris, R. L. Skaggs, M. S. Wigmosta, and A. M. Coleman, "A national-scale comparison of resource and nutrient demands for algae-based biofuel production by lipid extraction and hydrothermal liquefaction," Biomass and Bioenergy, vol. 64, pp. 276-290, May 2014.

[7] P. Biller and A. B. Ross, "Potential yields and properties of oil from the hydrothermal liquefaction of microalgae with different biochemical content," Bioresour. Technol., no. 102, pp. 215-225, 2011.

[8] F. Mairet, O. Bernard, P. Masci, T. Lacour, and A. Sciandra, "Modelling neutral lipid production by the microalga Isochrysis aff. galbana under nitrogen limitation.," Bioresour. Technol., vol. 102, no. 1, pp. 1429, Jan. 2011.

[9] E. D. Frank, A. Elgowainy, J. Han, and Z. Wang, "Life cycle comparison of hydrothermal liquefaction and lipid extraction pathways to renewable diesel from algae," Mitig. Adapt. Strateg. Glob. Chang., vol. 18, no. 1, pp. 137-158, Jun. 2012.

[10] U. Jena, K. C. Das, and J. R. Kastner, "Effect of operating conditions of thermochemical liquefaction on biocrude production from Spirulina platensis," Bioresour. Technol., vol. 102, no. 10, pp. 6221-6229, 2011.

[11] P. J. Valdez, M. C. Nelson, H. Y. Wang, X. N. Lin, and P. E. Savage, "Hydrothermal liquefaction of Nannochloropsis sp.: Systematic study of process variables and analysis of the product fractions," Biomass and Bioenergy, vol. 46, no. 0, pp. 317-331, 2012.

[12] T. Minowa and S. Sawayama, "A novel microalgal system for energy produciton with nitrogen cycling," Fuel, vol. 78, pp. 1213-1215, 1999. 
[13] K. Tsukahara, T. Kimura, T. Minowa, S. Sawayama, T. Yagishita, S. Inoue, T. Hanaoka, Y. Usui, and T. Ogi, "Microalgal cultivation in a solution recovered from the low-temperature catalytic gasification of the microalga," J. Biosci. Bioeng., vol. 91, no. 3, pp. 311-313, 2001.

[14] U. Jena, N. Vaidyanathan, S. Chinnasamy, and K. C. Das, "Evaluation of microalgae cultivation using recovered aqueous co-product from thermochemical liquefaction of algal biomass," Bioresour. Technol., vol. 102, no. 3, pp. 3380-3387, 2011.

[15] P. Biller, a. B. Ross, S. C. Skill, a. Lea-Langton, B. Balasundaram, C. Hall, R. Riley, and C. a. Llewellyn, "Nutrient recycling of aqueous phase for microalgae cultivation from the hydrothermal liquefaction process," Algal Res., vol. 1, no. 1, pp. 70-76, May 2012.

[16] M. Pham, L. Schideman, J. Scott, N. Rajagopalan, and M. J. Plewa, "Chemical and biological characterization of wastewater generated from hydrothermal liquefaction of Spirulina," Environ. Sci. Technol., vol. 47, no. 4, pp. 2131-2138, 2013.

[17] L. Garcia Alba, C. Torri, D. Fabbri, S. R. a. Kersten, and D. W. F. (Wim) Brilman, "Microalgae growth on the aqueous phase from Hydrothermal Liquefaction of the same microalgae," Chem. Eng. J., vol. 228, pp. 214-223, Jul. 2013.

[18] T. M. Brown, P. Duan, and P. E. Savage, "Hydrothermal liquefaction and gasification of Nannochloropsis sp.," Energy and Fuels, vol. 24, pp. 3639-3646, 2010.

[19] A. B. Ross, P. Biller, M. L. Kubacki, H. Li, A. Lea-Langton, and J. M. Jones, "Hydrothermal processing of microalgae using alkali and organic acids," Fuel, vol. 89, no. 9, pp. 2234-2243, 2010.

[20] P. J. Valdez, J. G. Dickinson, and P. E. Savage, "Characterization of product fractions from hydrothermal liquefaction of Nannochloropsis sp. and the influence of solvents," Energy and Fuels, vol. 25, no. 7, pp. 3235-3243, 2011.

[21] S. A. Allen, W. Clark, J. M. McCaffery, Z. Cai, A. Lanctot, P. J. Slininger, Z. L. Liu, and S. W. Gorsich, "Furfural induces reactive oxygen species accumulation and cellular damage in Saccharomyces cerevisiae," Biotechnol Biofuels, vol. 3, p. 2, 2010.

[22] H. Zheng, X. Wang, L. P. Yomano, K. T. Shanmugam, and L. O. Ingram, "Increase in furfural tolerance in ethanologenic Escherichia coli LY180 by plasmid-based expression of thyA," Appl Env. Microbiol, vol. 78, no. 12, pp. 4346-4352, 2012.

[23] X. Wang, L. P. Yomano, J. Y. Lee, S. W. York, H. Zheng, M. T. Mullinnix, K. T. Shanmugam, and L. O. Ingram, "Engineering furfural tolerance in Escherichia coli improves the fermentation of lignocellulosic sugars into renewable chemicals.," Proc. Natl. Acad. Sci. U. S. A., vol. 110, no. 10, pp. 4021-6, Mar. 2013.

[24] S. H. Choi and M. B. Gu, "Phenolic toxicity--detection and classification through the use of a recombinant bioluminescent Escherichia coli," Environ. Toxicol. Chem., vol. 20, no. 2, pp. 248-255, 2001.

[25] C. Roma-Rodrigues, P. M. Santos, D. Benndorf, E. Rapp, and I. Sa-Correia, "Response of Pseudomonas putida KT2440 to phenol at the level of membrane proteome," J Proteomics, vol. 73, no. 8, pp. 1461-1478, 2010.

[26] L. Sundstrom, S. Larsson, and L. J. Jonsson, "Identification of Saccharomyces cerevisiae genes involved in the resistance to phenolic fermentation inhibitors," Appl. Biochem. Biotechnol., vol. 161, no. 1-8, pp. 106$115,2010$. 
[27] M. P. Almario, L. H. Reyes, and K. C. Kao, "Evolutionary engineering of Saccharomyces cerevisiae for enhanced tolerance to hydrolysates of lignocellulosic biomass.," Biotechnol. Bioeng., vol. 110, no. 10, pp. 2616-23, Oct. 2013.

[28] T. Hasunuma, T. Sanda, R. Yamada, K. Yoshimura, J. Ishii, and A. Kondo, "Metabolic pathway engineering based on metabolomics confers acetic and formic acid tolerance to a recombinant xylose-fermenting strain of Saccharomyces cerevisiae," Microb Cell Fact, vol. 10, no. 1, p. 2, 2011.

[29] M. Z. Ding, X. Wang, Y. Yang, and Y. J. Yuan, "Metabolomic study of interactive effects of phenol, furfural, and acetic acid on Saccharomyces cerevisiae," OMICS, vol. 15, no. 10, pp. 647-653, 2011.

[30] N. R. Sandoval, T. Y. Mills, M. Zhang, and R. T. Gill, "Elucidating acetate tolerance in E. coli using a genome-wide approach," Metab Eng, vol. 13, no. 2, pp. 214-224, 2011.

[31] G. Cao, E. Ximenes, N. N. Nichols, L. Zhang, and M. Ladisch, "Biological abatement of cellulase inhibitors.," Bioresour. Technol., vol. 146, pp. 604-10, Oct. 2013.

[32] F. Koopman, N. Wierckx, J. H. de Winde, and H. J. Ruijssenaars, "Identification and characterization of the furfural and 5-(hydroxymethyl)furfural degradation pathways of Cupriavidus basilensis HMF14," Proc Natl Acad Sci U S A, vol. 107, no. 11, pp. 4919-4924, 2010.

[33] A. M. Feist, C. S. Henry, J. L. Reed, M. Krummenacker, A. R. Joyce, P. D. Karp, L. J. Broadbelt, V. Hatzimanikatis, and B. Ø. Palsson, "A genome-scale metabolic reconstruction for Escherichia coli K-12 MG1655 that accounts for 1260 ORFs and thermodynamic information," Mol. Syst. Biol., vol. 3, no. 121, 2007.

[34] H. Alper and G. Stephanopoulos, "Engineering for biofuels: exploiting innate microbial capacity or importing biosynthetic potential?," Nat. Rev. Microbiol., vol. 7, 2009.

[35] R. Radakovits, R. E. Jinkerson, A. Darzins, and M. C. Posewitz, "Genetic Engineering of Algae for Enhanced Biofuel Production,” Eukaryot. Cell, vol. 9, no. 4, pp. 486-501, 2010.

[36] N. M. Courchesne, A. Parisien, B. Wang, and C. Q. Lan, "Enhancement of lipid production using biochemical, genetic and transcription factor engineering approaches," J. Biotechnol., 2009.

[37] S. Atsumi and J. C. Liao, "Metabolic engineering for advanced biofuels production from Escherichia coli," Curr. Opin. Biotechnol., vol. 19, pp. 414-419, 2008.

[38] Q. Yang, J. Wang, H. Wang, X. Chen, S. Ren, X. Li, Y. Xu, H. Zhang, and X. Li, "Evolution of the microbial community in a full-scale printing and dyeing wastewater treatment system.," Bioresour. Technol., vol. 117, pp. 155-63, Aug. 2012.

[39] S. S. Fong, A. R. Joyce, and B. Ø. Palsson, "Parallel adaptive evolution cultures of Escherichia coli lead to convergent growth phenotypes with different gene expression states," Genome Res., vol. 15, pp. 1365-1372, 2005 .

[40] Z. D. Blount, C. Z. Borland, and R. E. Lenski, "Historical contingency and the evolution of a key innovation in an experimental population of Eschericia coli," Proc. Natl. Acad. Sci., vol. 105, no. 23, pp. 7899-7906, 2008.

[41] H. Gu, J. Zhang, and J. Bao, "Inhibitor analysis and adaptive evolution of Saccharomyces cerevisiae for simultaneous saccharification and ethanol fermentation from industrial waste corncob residues.," Bioresour. Technol., vol. 157, pp. 6-13, Apr. 2014. 
[42] J. J. Minty, A. a Lesnefsky, F. Lin, Y. Chen, T. a Zaroff, A. B. Veloso, B. Xie, C. a McConnell, R. J. Ward, D. R. Schwartz, J.-M. Rouillard, Y. Gao, E. Gulari, and X. N. Lin, "Evolution combined with genomic study elucidates genetic bases of isobutanol tolerance in Escherichia coli.," Microb. Cell Fact., vol. 10, no. 1, p. 18, Jan. 2011.

[43] E. R. Mardis, "The impact of next-generation sequencing technology on genetics.," Trends Genet., vol. 24, no. 3, pp. 133-41, Mar. 2008.

[44] F. R. Blattner, "The Complete Genome Sequence of Escherichia coli K-12," Science (80-. )., vol. 277, no. 5331, pp. 1453-1462, Sep. 1997.

[45] O. Morozova, M. Hirst, and M. a Marra, "Applications of new sequencing technologies for transcriptome analysis.," Annu. Rev. Genomics Hum. Genet., vol. 10, pp. 135-51, Jan. 2009.

[46] N. Cloonan, A. R. R. Forrest, G. Kolle, B. B. a Gardiner, G. J. Faulkner, M. K. Brown, D. F. Taylor, A. L. Steptoe, S. Wani, G. Bethel, A. J. Robertson, A. C. Perkins, S. J. Bruce, C. C. Lee, S. S. Ranade, H. E. Peckham, J. M. Manning, K. J. McKernan, and S. M. Grimmond, "Stem cell transcriptome profiling via massive-scale mRNA sequencing.," Nat. Methods, vol. 5, no. 7, pp. 613-9, Jul. 2008.

[47] N. Nagaraj, J. R. Wisniewski, T. Geiger, J. Cox, M. Kircher, J. Kelso, S. Pääbo, and M. Mann, "Deep proteome and transcriptome mapping of a human cancer cell line.," Mol. Syst. Biol., vol. 7, no. 548, p. 548, Jan. 2011.

[48] R. Landstorfer, S. Simon, S. Schober, D. Keim, S. Scherer, and K. Neuhaus, "Comparison of strand-specific transcriptomes of enterohemorrhagic Escherichia coli O157:H7 EDL933 (EHEC) under eleven different environmental conditions including radish sprouts and cattle feces.," BMC Genomics, vol. 15, no. 1, p. 353, Jan. 2014.

[49] J. Cole, "Interactions between bacteria and algae in aquatic ecosystems," Annu. Rev. Ecol. Aystematics, vol. 13, pp. 291-314, 1982.

[50] M. Gavrilescu and Y. Chisti, "Biotechnology-a sustainable alternative for chemical industry.," Biotechnol. Adv., vol. 23, no. 7-8, pp. 471-99, Nov. 2005.

[51] M. Packer, "Algal capture of carbon dioxide; biomass generation as a tool for greenhouse gas mitigation with reference to New Zealand energy strategy and policy," Energy Policy, vol. 37, no. 9, pp. 3428-3437, 2009.

[52] W. Mulbry, S. Kondrad, C. Pizarro, and E. Kebede-Westhead, "Treatment of dairy manure effluent using freshwater algae: Algal productivity and recovery of manure nutrients using pilot-scale algal turf scrubbers," Bioresour. Technol., vol. 99, no. 17, pp. 8137-8142, 2008.

[53] Y. Dote, S. Sawayama, S. Inoue, T. Minowa, and S. Yokoyama, "Recovery of liquid fuel from hydrocarbonrich microalgae by thermochemical liquefaction," Fuel, vol. 73, no. 12, pp. 1855-1857, 1994.

[54] C. Torri, L. Garcia Alba, C. Samorì, D. Fabbri, and D. W. F. Brilman, "Hydrothermal treatment (HTT) of microalgae: Detailed molecular characterization of HTT oil in view of HTT mechanism elucidation," Energy and Fuels, vol. 26, no. 1, pp. 658-671, 2012.

[55] T. Minowa, S. Yokoyama, M. Kishimoto, and T. Okakura, "Oil production from algal cells of Dunaliella tertiolecta by direct thermochemical liquefaction," Fuel, vol. 74, no. 12, pp. 1735-1738, 1995. 
[56] Y. F. Yang, C. P. Feng, Y. Inamori, and T. Maekawa, "Analysis of energy conversion characteristics in liquefaction of algae," Resour. Conserv. Recycl., vol. 43, no. 1, pp. 21-33, 2004.

[57] P. Duan and P. E. Savage, "Hydrothermal Liquefaction of a Microalga with Heterogeneous Catalysts," Ind. Eng. Chem. Res., vol. 50, no. 1, pp. 52-61, 2011.

[58] L. Garcia Alba, C. Torri, C. Samorì, J. van der Spek, D. Fabbri, S. R. A. Kersten, and D. W. F. (Wim) Brilman, "Hydrothermal Treatment (HTT) of Microalgae: Evaluation of the Process As Conversion Method in an Algae Biorefinery Concept," Energy \& Fuels, vol. 26, no. 1, pp. 642-657, 2012.

[59] A. A. Peterson, F. Vogel, R. P. Lachance, M. Froling, M. J. Antal, and J. W. Tester, "Thermochemical biofuel productionin hydrothermal media: A review of sub- and supercritical water technologies," Energy Environ. Sci., no. 1, pp. 32-65, 2008.

[60] L. Zhang, C. (Charles) Xu, and P. Champagne, "Overview of recent advances in thermo-chemical conversion of biomass," Energy Convers. Manag., vol. 51, no. 5, pp. 969-982, 2010.

[61] S. O. Lourenço, E. Barbarino, P. L. Lavín, U. M. Lanfer Marquez, and E. Aidar, "Distribution of intracellular nitrogen in marine microalgae: Calculation of new nitrogen-to-protein conversion factors," Eur. J.Phycol., vol. 39, no. 1, pp. 17-32, 2004.

[62] P. H. Pfromm, V. Amanor-Boadu, and R. Nelson, "Sustainability of algae derived biodiesel: A mass balance approach," Bioresour. Technol., vol. 102, no. 2, pp. 1185-1193, 2011.

[63] F. Touratier, J. G. Field, and C. L. Moloney, "A stoichiometric model relating growth substrate quality (C:N:P ratios) to N:P ratios in the products of heterotrophic release and excretion," Ecol. Modell., vol. 139, no. 2-3, pp. 265-291, Apr. 2001.

[64] J. C. Valderrama, "The Simultaneous Analysis of Total Nitrogen and Total Phosphorus in Natural-Waters," Mar. Chem., vol. 10, no. 2, pp. 109-122, 1981.

[65] G. E. Pacey, S. Steig, B. R. Fisher, D. C. Hillman, O. B. Mathre, and J. W. O'Dell, Standard methods for the examination of wastewater. Washington DC: American Public Health Association, 1999, pp. 139-145.

[66] H. C. Greenwell, L. M. L. Laurens, R. J. Shields, R. W. Lovitt, and K. J. Flynn, "Placing microalgae on the biofuels priority list: A review of the technological challenges," J. R. Soc. Interface, vol. 7, no. 46, pp. 703$726,2010$.

[67] A. F. Clarens, E. P. Resurreccion, M. A. White, and L. M. Colosi, "Environmental life cycle comparison of algae to other bioenergy feedstocks," Environ. Sci. Technol., vol. 44, no. 5, pp. 1813-1819, 2010.

[68] P. Biller, A. B. Ross, S. C. Skill, A. Lea-Langton, B. Balasundaram, C. Hall, R. Riley, and C. A. Llewellyn, "Nutrient recycling of aqueous phase for microalgae cultivation from the hydrothermal liquefaction process," Algal Res., vol. 1, no. 1, pp. 70-76, 2012.

[69] M. Bajaj, C. Gallert, and J. Winter, "Biodegradation of high phenol containing synthetic wastewater by an aerobic fixed bed reactor," Bioresour. Technol., vol. 99, no. 17, pp. 8376-8381, 2008.

[70] R. Davis, A. Aden, and P. T. Pienkos, "Techno-economic analysis of autotrophic microalgae for fuel production," Appl. Energy, vol. 88, no. 10, pp. 3524-3531, 2011. 
[71] S. U. Nwachukwu, "Bioremediation of sterile agricultural soils polluted with crude petroleum by application of the soil bacterium Pseudomonas putida with inorganic nutrient supplementations," Curr. Microbiol., vol. 42, pp. 231-236, 2001.

[72] C. C. Cleveland and D. Liptzin, "C:N:P stoichiometry in soil: Is there a 'Redfield ratio' for the microbial biomass?," Biogeochemistry, vol. 85, no. 3, pp. 235-252, 2007.

[73] A. C. Redfield, "The Biological Control of Chemical Factors in the Environment," Am. Sci., vol. 46, no. 3 , pp. 205-221, 1958.

[74] W. Xue, D. Fan, L. Shang, C. Zhu, X. Ma, X. Zhu, and Y. Yu, "Effects of acetic acid and its assimilation in fed-batch cultures of recombinant Escherichia coli containing human-like collagen cDNA," J. Biosci. Bioeng., vol. 109, no. 3, pp. 257-261, 2010.

[75] B. Volkmer and M. Heinemann, "Condition-dependent cell volume and concentration of Escherichia coli to facilitate data conversion for systems biology modeling," PLoS One, vol. 6, no. 7, p. e23126, 2011.

[76] Z. Du, B. Hu, A. Shi, X. Ma, Y. Cheng, P. Chen, Y. Liu, X. Lin, and R. Ruan, "Cultivation of a microalga Chlorella vulgaris using recycled aqueous phase nutrients from hydrothermal carbonization process.," Bioresour. Technol., vol. 126, pp. 354-7, Dec. 2012.

[77] M. Nelson, L. Zhu, A. Thiel, Y. Wu, M. Guan, J. Minty, H. Y. Wang, and X. N. Lin, "Microbial utilization of aqueous co-products from hydrothermal liquefaction of microalgae Nannochloropsis oculata.," Bioresour. Technol., vol. 136, pp. 522-8, May 2013.

[78] P. J. Valdez, M. C. Nelson, J. L. Faeth, H. Y. Wang, X. N. Lin, and P. E. Savage, "Hydrothermal Liquefaction of Bacteria and Yeast Monocultures," 2013.

[79] N. D. Orfield, A. J. Fang, P. J. Valdez, M. C. Nelson, P. E. Savage, X. N. Lin, and G. A. Keoleian, "Life Cycle Design of an Algal Biore fi nery Featuring Hydrothermal Liquefaction: E ff ect of Reaction Conditions and an Alternative Pathway Including Microbial Regrowth," 2014.

[80] C. D. Herring, A. Raghunathan, et al., and B. Ø. Palsson, "Comparative genome sequencing of Escherichia coli allows observation of bacterial evolution on a laboratory timescale," Nat. Genet., vol. 38, no. 12, pp. 1406-1412, 2006.

[81] C. Liu, D. Yong, D. Yu, and S. Dong, "Cell-based biosensor for measurement of phenol and nitrophenols toxicity," Talanta, vol. 84, no. 3, pp. 766-770, 2011.

[82] J. I. Jiménez, B. Miñambres, J. L. García, and E. Díaz, "Genomic analysis of the aromatic catabolic pathways from Pseudomonas putida KT2440.," Environ. Microbiol., vol. 4, no. 12, pp. 824-41, Dec. 2002.

[83] L. Reitzer, "Nitrogen assimilation and global regulation in Escherichia coli.," Annu. Rev. Microbiol., vol. 57, pp. 155-76, Jan. 2003.

[84] K. Sathasivan, G. W. Haughn, and N. Murai, "Molecular Basis of Imidazolinone Herbicide Resistance in Arabidopsis thaliana var Columbia.," Plant Physiol., vol. 97, no. 3, pp. 1044-50, Nov. 1991.

[85] Ormo M. et al, "Crystal structure of a complex of Escherichia coli glycerol kinase and an allosteric effector fructose 1,6-bisphosphate," Biochemistry, vol. 37, pp. 16565-16572, 1998. 
[86] Anderson M. J. et al, "Crystal structure of a hyperactive Escherichia coli glycerol kinase mutant Gly230Asp obtained using microfluidic crystallization devices," Biochemistry, vol. 46, pp. 5722-5731, 2007.

[87] D. De Biase, a Tramonti, F. Bossa, and P. Visca, "The response to stationary-phase stress conditions in Escherichia coli: role and regulation of the glutamic acid decarboxylase system.," Mol. Microbiol., vol. 32, no. 6, pp. 1198-211, Jun. 1999.

[88] J. K. Flynn, K. Davidson, and A. Cunningham, "Relations between carbon and nitrogen during growth of Nannochloropsisoculata (Droop) Hibberd under continuous illumination," New Phytol., vol. 125, no. 4, p. $7170722,1993$.

[89] Y. Y. Chang and J. E. Cronan, "Membrane cyclopropane fatty acid content is a major factor in acid resistance of Escherichia coli.," Mol. Microbiol., vol. 33, no. 2, pp. 249-59, Jul. 1999.

[90] a R. Strøm and I. Kaasen, "Trehalose metabolism in Escherichia coli: stress protection and stress regulation of gene expression.," Mol. Microbiol., vol. 8, no. 2, pp. 205-10, Apr. 1993.

[91] R. Hengge-aronis, "Survival of Hunger and Stress : The Rde of rpoS in Early SW Gene Reguktiin Ph," vol. 72, pp. 165-166, 1993.

[92] E. N. Miller, L. R. Jarboe, P. C. Turner, P. Pharkya, L. P. Yomano, S. W. York, D. Nunn, K. T. Shanmugam, and L. O. Ingram, "Furfural inhibits growth by limiting sulfur assimilation in ethanologenic Escherichia coli strain LY180.," Appl. Environ. Microbiol., vol. 75, no. 19, pp. 6132-41, Oct. 2009.

[93] A. J. McBroom, A. P. Johnson, S. Vemulapalli, and M. J. Kuehn, "Outer membrane vesicle production by Escherichia coli is independent of membrane instability.," J. Bacteriol., vol. 188, no. 15, pp. 5385-92, Aug. 2006.

[94] F. C. Neidhardt, P. L. Bloch, and D. F. Smith, "Culture medium for enterobacteria," J. Bacteriol., vol. 119, no. 3, pp. 736-747, 1974.

[95] M. J, "Growth of Bacterial Cultures," Annu. Rev. Microbiol., vol. 3, pp. 371-394, 1949.

[96] H. H. Wang, F. J. Isaacs, P. A. Carr, Z. Z. Sun, G. Xu, C. R. Forest, and G. M. Church, "Programming cells by multiplex genome engineering and accelerated evolution," Nature, vol. 460, 2009.

[97] B. Langmead, C. Trapnell, M. Pop, and S. L. Salzberg, "Ultrafast and memory efficient alignment of short DNA sequences to the human genome. Genome Biology," Genome Biol., vol. 10, no. 3, p. R25, 2009.

[98] C. Trapnell, L. Pachter, and S. L. Salzberg, "TopHat: discovering splice junctions with RNA-Seq.." Bioinformatics, vol. 25, no. 9, pp. 1105-1111, 2009.

[99] C. Trapnell, D. Hendrickson, S. Sauvageau, L. Goff, J. Rinn, and L. Pachter, "Differential analysis of gene regulation at transcript resolution with RNA-seq," Nat. Biotechnol.

[100] S. Grossmann, S. Bauer, P. N. Robinson, and M. Vingron, "Improved detection of overrepresentation of Gene-Ontology annotations with parent child analysis.," Bioinformatics, vol. 23, no. 22, pp. 3024-31, Nov. 2007.

[101] F. Leroy and L. De Vuyst, "Lactic acid bacteria as functional starter cultures for the food fermentation industry," Trends Food Sci. Technol., vol. 15, no. 2, pp. 67-78, Feb. 2004. 
[102] C. Angerbauer, M. Siebenhofer, M. Mittelbach, and G. M. Guebitz, "Conversion of sewage sludge into lipids by Lipomyces starkeyi for biodiesel production," Bioresour. Technol., vol. 99, no. 8, pp. 3051-3056, 2008.

[103] S. Huffer, C. M. Roche, H. W. Blanch, and D. S. Clark, "Escherichia coli for biofuel production: Bridging the gap from promise to practice," Trends Biotechnol., vol. 30, no. 10, pp. 538-545, 2012.

[104] C. Ratledge and Z. Cohen, "Microbial and algal oils: Do they have a future for biodiesel or as commodity oils?," Lipid Technol., vol. 20, no. 7, pp. 155-160, 2008.

[105] B. D. Wahlen, M. R. Morgan, A. T. McCurdy, R. M. Willis, M. D. Morgan, D. J. Dye, B. Bugbee, B. D. Wood, and L. C. Seefeldt, "Biodiesel from microalgae, yeast, and bacteria: Engine performance and exhaust emissions," Energy and Fuels, vol. 27, no. 1, pp. 220-228, 2013.

[106] N. J. Horan, “No Title,” Biol. Wastewater Treat. Syst., 1990.

[107] D. R. Vardon, B. K. Sharma, J. Scott, G. Yu, Z. Wang, L. Schideman, Y. Zhang, and T. J. Strathmann, "Chemical properties of biocrude oil from the hydrothermal liquefaction of Spirulina algae, swine manure, and digested anaerobic sludge," Bioresour. Technol., vol. 102, no. 17, pp. 8295-8303, 2011.

[108] M. Murakami, S. -y. Yokoyama, T. Ogi, and K. Koguchi, "Direct liquefaction of activated sludge from aerobic treatment of effluents from the cornstarch industry," Biomass, vol. 23, no. 3, pp. 215-228, 1990.

[109] C. Xu and J. Lancaster, "Conversion of secondary pulp/paper sludge powder to liquid oil products for energy recovery by direct liquefaction in hot-compressed water," Water Res., vol. 42, no. 6-7, pp. 1571$1582,2008$.

[110] D. R. Vardon, B. K. Sharma, J. Scott, G. Yu, Z. Wang, L. Schideman, Y. Zhang, and T. J. Strathmann, "Chemical properties of biocrude oil from the hydrothermal liquefaction of Spirulina algae, swine manure, and digested anaerobic sludge," Bioresour. Technol., vol. 102, no. 17, pp. 8295-8303, 2011.

[111] W. J. Catallo and J. L. Comeaux, "Reductive hydrothermal treatment of sewage sludge," Waste Manag., vol. 28, no. 11, pp. 2213-2219, 2008.

[112] G. W. Roberts, M.-O. P. Fortier, B. S. M. Sturm, and S. M. Stagg-Williams, "Promising pathway for algal biofuels through wastewater cultivation and hydrothermal conversion," Energy and Fuels, vol. 27, no. 2, pp. 857-867, 2013.

[113] J. L. Faeth, P. J. Valdez, and P. E. Savage, "Fast hydrothermal liquefaction of nannochloropsis sp. to produce biocrude," Energy and Fuels, vol. 27, no. 3, pp. 1391-1398, 2013.

[114] L. S. Gronenberg, R. J. Marcheschi, and J. C. Liao, "Next generation biofuel engineering in prokaryotes," Curr. Opin. Chem. Biol., vol. 17, no. 3, pp. 462-471, 2013.

[115] K. M. Fagerbakke, M. Heldal, and S. Norland, "Content of carbon, nitrogen, oxygen, sulfur and phosphorus in native aquatic and cultured bacteria," Aquat. Microb. Ecol., vol. 10, no. 1, pp. 15-27, 1996.

[116] H. C. Lange and J. J. Heijnen, "Statistical reconciliation of the elemental and molecular biomass composition of Saccharomyces cerevisiae," Biotechnol. Bioeng., vol. 75, no. 3, pp. 334-344, 2001.

[117] E. W. Becker, "Micro-algae as a source of protein," Biotechnol. Adv., vol. 25, no. 2, pp. 207-210, 2007. 
[118] H. Tao, C. Bausch, C. Richmond, F. R. Blattner, and T. Conway, "Functional genomics: expression analysis of Escherichia coli growing on minimal and rich media.," J. Bacteriol., vol. 181, no. 20, pp. 6425-40, Oct. 1999.

[119] N. Sato, A. T. Quitain, K. Kang, H. Daimon, and K. Fujie, "Reaction kinetics of amino acid decomposition in high-temperature and high-pressure water," Ind. Eng. Chem. Res., vol. 43, no. 13, pp. 3217-3222, 2004.

[120] J. G. Speight, “No Title,” Chem. Technol. Pet., 1991.

[121] K. Sasaki, M. Watanabe, T. Tanaka, T., "Biosynthesis, biotechnological production and applications of 5aminolevulinic acid," Appl. Microbiol. Biotechnol., vol. 58, no. 1, pp. 23-29, Jan. 2002.

[122] V. A. Yaylayan and A. Huyghues-Despointes, "Identification of per-O-(trimethylsilyl) derivatives of aldoses generated from thermal decomposition of N-(1-deoxy-D-fructopyranos-1-y1)proline: Reversibility of the Amadori rearrangement," Carbohydr. Res., vol. 286, pp. 179-187, 1996.

[123] R. B. Levine, A. A. Bollas, M. D. Durham, and P. E. Savage, "Triflate-catalyzed (trans)esterification of lipids within carbonized algal biomass," Bioresour. Technol., vol. 111, pp. 222-229, 2012.

[124] T. Lewis, P. D. Nichols, and T. A. McMeekin, "Evaluation of extraction methods for recovery of fatty acids from lipid-producing microheterotrophs," J. Microbiol. Methods, vol. 43, no. 2, pp. 107-116, 2000.

[125] M. Piorreck, K.-H. Baasch, and P. Pohl, "Biomass production, total protein, chlorophylls, lipids and fatty acids of freshwater green and blue-green algae under different nitrogen regimes," Phytochemistry, vol. 23, no. 2, pp. 207-216, 1984.

[126] A. M. A. Pistorius, W. J. DeGrip, and T. A. Egorova-Zachernyuk, "Monitoring of biomass composition from microbiological sources by means of FT-IR spectroscopy," Biotechnol. Bioeng., vol. 103, no. 1, pp. 123-129, 2009.

[127] K. Annamalai, J. M. Sweeten, and S. C. Ramalingam, "Estimation of gross heating values of biomass fuels," Trans ASAE, vol. 30, no. 4, pp. 1205-1208, 1987.

[128] J. Ferrell and V. Sarisky-Reed, "National Algal Biofuels Technology Roadmap," U.S. Dep. energy, Off. Energy Effic. Renew. Energy, Biomass Program., 2010.

[129] M. T. Croft, A. D. Lawrence, E. Raux-Deery, M. J. Warren, and A. G. Smith, “Algae acquire vitamin B12 through a symbiotic relationship with bacteria.," Nature, vol. 438, no. 7064, pp. 90-3, Nov. 2005.

[130] G. Mitchell and D. A. Kiefer, "Chlorophyll a specific absorption and fluorescence excitation spectra for fight-limited phytoplankton,” Deep. Res., vol. 35, no. 5, pp. 639-663, 1988. 